\title{
Beauty and the Brand: A Digital Ethnography of Social Capital and Authenticity of Digital Beauty Influencers through Monetization Activities on YouTube
}

\author{
Hannah R. Gnegy
}

Follow this and additional works at: https://researchrepository.wvu.edu/etd

\section{Recommended Citation}

Gnegy, Hannah R., "Beauty and the Brand: A Digital Ethnography of Social Capital and Authenticity of Digital Beauty Influencers through Monetization Activities on YouTube" (2017). Graduate Theses, Dissertations, and Problem Reports. 5683.

https://researchrepository.wvu.edu/etd/5683

This Dissertation is protected by copyright and/or related rights. It has been brought to you by the The Research Repository @ WVU with permission from the rights-holder(s). You are free to use this Dissertation in any way that is permitted by the copyright and related rights legislation that applies to your use. For other uses you must obtain permission from the rights-holder(s) directly, unless additional rights are indicated by a Creative Commons license in the record and/ or on the work itself. This Dissertation has been accepted for inclusion in WVU Graduate Theses, Dissertations, and Problem Reports collection by an authorized administrator of The Research Repository @ WVU.

For more information, please contact researchrepository@mail.wvu.edu. 
BEAUTY AND THE BRAND: A DIGITAL ETHNOGRAPHY OF SOCIAL CAPITAL AND AUTHENTICITY OF DIGITAL BEAUTY INFLUENCERS THROUGH MONETIZATION ACTIVITIES ON YOUTUBE

Hannah R. Gnegy

\author{
Dissertation submitted \\ to the Davis College of Agriculture, Natural Resources, and Design \\ at West Virginia University \\ in partial fulfillment of the requirements for the degree of \\ Doctor of Philosophy in \\ Human and Community Development \\ Kathryn Baker - Jones, Ph.D., Chair \\ Jennifer Yang, Ph.D. \\ Jessica Blythe, Ph.D. \\ Christine Rittenour, Ph.D. \\ Nora MacDonald, M.S. \\ School of Design and Community Development \\ Division of Design and Merchandising \\ Morgantown, West Virginia \\ June 2017
}

Keywords: Digital influencers, social capital, authenticity, viewer feedback Copyright 2017 Hannah Gnegy 


\begin{abstract}
BEAUTY AND THE BRAND: A DIGITAL ETHNOGRAPHY OF SOCIAL CAPITAL AND AUTHENTICITY OF DIGITAL BEAUTY INFLUENCERS THROUGH MONETIZATION ACTIVITIES ON YOUTUBE
\end{abstract}

\begin{abstract}
Hannah R. Gnegy
This dissertation explored the maintenance of social capital, projection of authenticity, alignment of beauty brands with the brand-as-person, and communicative practices of beauty influencers through a digital ethnography of YouTube beauty community. This research addressed how monetization practices by popular beauty influencers could affect the social constructs required in maintaining their position in the digital community. As beauty influencers continue to gain notoriety and engage in monetization activities through their standing on YouTube, it was important to address how the social practices utilized to build this notoriety were impacted by commoditization of content, toward understanding the sustainability of these practices for influencers and the beauty brands with whom they partner. A digital ethnography, utilizing an inductive content analysis and framework analysis, served as the method by which assessment of influencer projections and viewer reaction, within the cultural confines of the digital community, could be assessed. This research found that influencer projections were all impacted by the type of content. When influencers engaged in sponsored posts, viewers noted disparities in each of the constructs explored in this study, suggesting certain monetization activities can lower reputation engagement in the community. If influencers wish to engage in these monetization practices, they should be upfront with viewers about their intentions, choosing partnerships that are built through time and consistency. In doing so, influencers then are able to situate themselves as being genuine and honest with viewers, cementing their status in the community, while still benefitting personally and financially from monetization activities.
\end{abstract}




\section{ACKNOWLEDGEMENTS}

The completion of this work was made possible through the help, support, and encouragement of my family, friends, mentors, and academic units. The appreciation I have for every individual and organization involved in this process truly cannot be defined by any words, but I am going to try.

First, thank you to the Fashion, Dress and Merchandising program within the Davis College of Agriculture, Natural Resources, and Design at West Virginia University for the opportunity to serve as a graduate assistant and for the continued financial support of my studies, throughout my doctoral program. Thank you also to the School of Design and Community Development staff and faculty members for your continued support and guidance in pursuing this degree. Each of you has left an impression upon me and I am humbled.

It is with the sincerest level of gratitude and appreciation I thank Dr. Kathryn BakerJones. Without you, I would still be sitting in my office trying to figure out how to navigate this path via the Internet. Thank you for dedicating your time to the countless hours of meetings and frantic e-mail messages that accompanied this process. You have taught me far more beyond how to write a dissertation. You have always been my advocate, helping me to navigate difficult paths and showing me how to achieve my goals in a thoughtful, yet purposeful manner. You have truly served as my role model and I know I wouldn't be half the student and researcher I am without your guidance.

To Professor Nora MacDonald and Dr. Jennifer Yang, thank you for being a support system for the past three years. Thank you also for helping me to become a stronger researcher and writer. Your knowledge and passion for fashion dress and merchandising is immeasurable. To Dr. Jessica Blythe and Dr. Christine Rittenour, thank you for providing me with a second 
perspective from which to view my research. Thank you for always being available to talk and offering guidance beyond what was ever required. Thank you all for teaching me skills that will last a lifetime.

To my fellow graduate students, please know you each hold an extremely special place in my heart. Nobody else will ever know the deep conversations that happen in the "fish bowl" offices. To Kristina and Rebekah, sharing an office with you both was one of the best experiences of my graduate education. The countless hours of talking about research, classes, and life in general will stay with me forever. I want to extend my deepest thanks to you, Angie. I never thought I would gain one of my best friends through graduate school in Allen Hall. We experienced it all together, from the highest of highs to the lowest of lows, and everything in between. We have flown across the country together and I still find new things to giggle with you about every time we see each other. You are an incredible person and I am infinitely blessed to have you in my life. To all my other graduate school colleagues, know I am so proud of everything you have accomplished and am honored to call you my cohort and friends.

To my family: there is not enough space in even the largest book to express the thanks I owe and love I have for each of you. To mom and dad, I love you both so much. I truly was blessed with the greatest parents imaginable. When I came to you and said I wanted to attend graduate school, you didn't question why. You just told me how proud you were of me for making that decision. Every time I think about what amazing role models you both are, it brings tears to my eyes. Your support is the one thing which has truly kept me afloat. There is no way to repay you for everything you have provided me in life and for that I owe you my deepest gratitude. 
Finally, to my dear husband, Doug, you should earn an award for being the most tolerant human being ever. You have been here through every step. You have seen me crying over my laptop, overjoyed when I passed my exams, and generally just worried about what the future holds. And yet regardless of what emotion I was feeling each day, you always made me laugh (usually with a context-specific Internet meme) and cheered me on. I am excited to begin this next chapter of our lives together and know everything will be incredible, with you by my side. Also, thank you for washing countless loads of laundry and becoming a fantastic chef.

To each of you, this journey would not have been possible without your support. Thank you for your guidance. You each hold a very special place in my heart. 


\section{TABLE OF CONTENTS}

ACKNOWLEDGEMENTS ....................................................................................................... iii

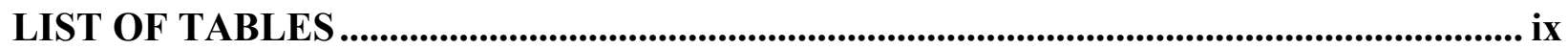

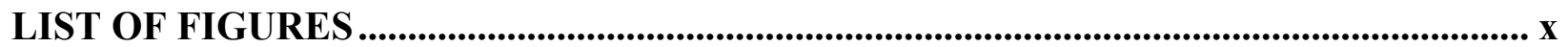

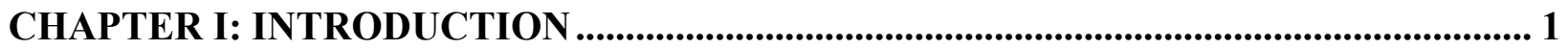

Gap in Knowledge - Identifying a Problem .................................................................... 2

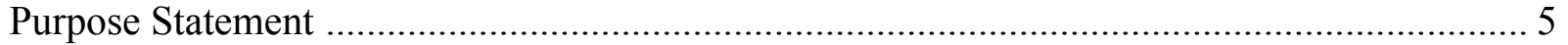

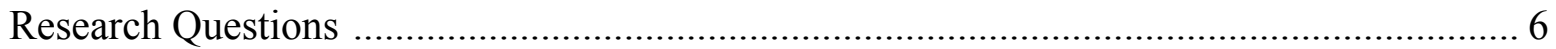

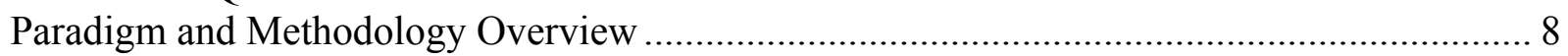

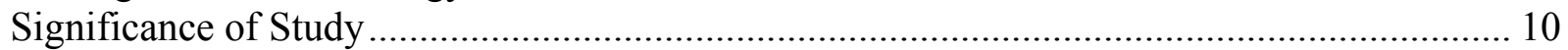

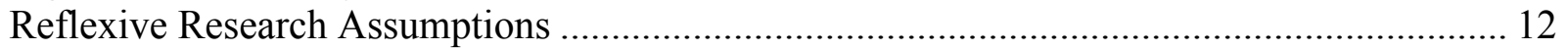

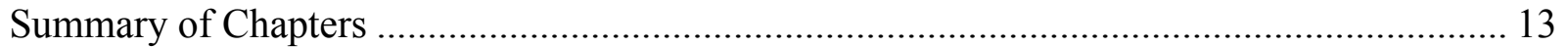

CHAPTER II: LITERATURE REVIEW .................................................................... 16

Technological Change - The Introduction of the Web ........................................................ 16

Affordances of Social Network Sites ………………................................................... 19

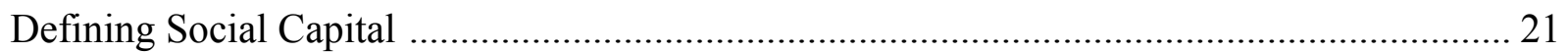

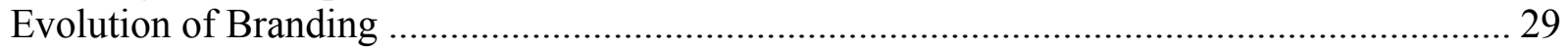

Branding-Extended Identity Measures ........................................................................ 32

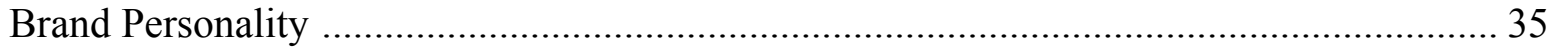

Brand Migration to Social Network Sites ........................................................................... 37

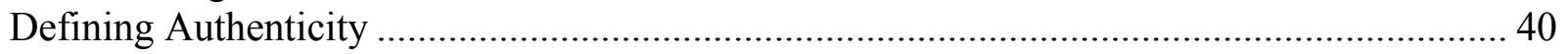

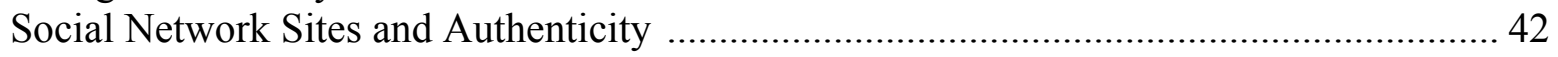

Consumer-Generated Advertising and Branding ……….................................................... 44

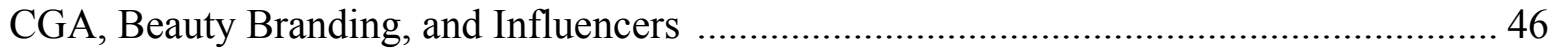

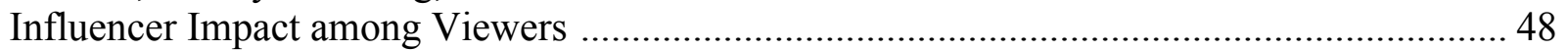

Summary of Study Based on Literature Review ............................................................... 50

CHAPTER III: METHODOLOGY ..................................................................................... 52

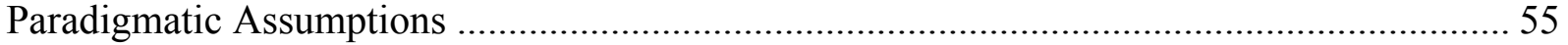

Selection of Qualitative Methodology ……………........................................................ 57

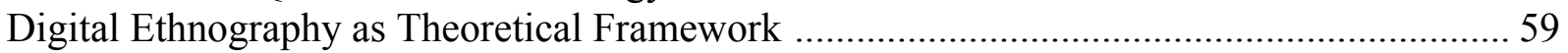

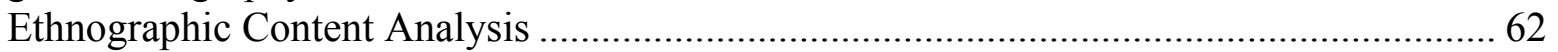

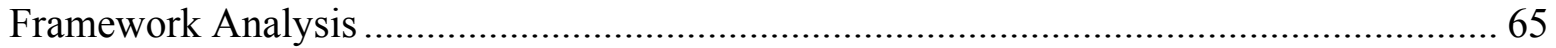

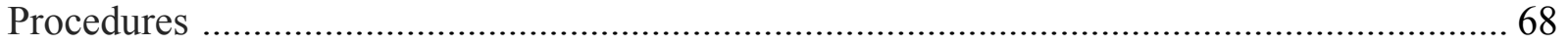

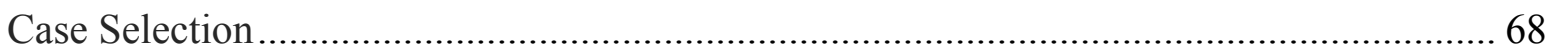

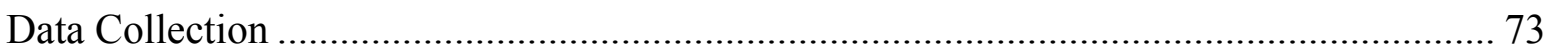

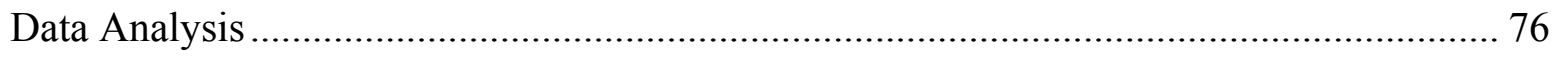

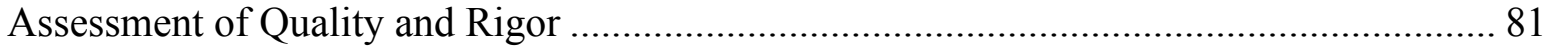

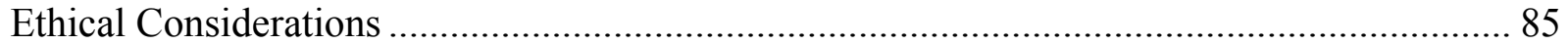

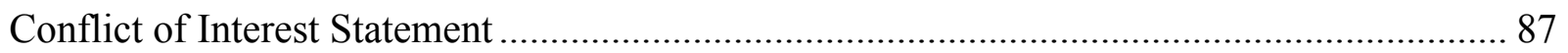




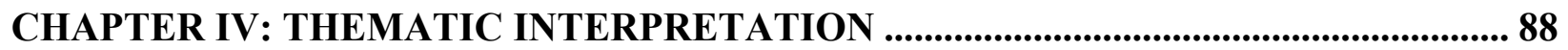

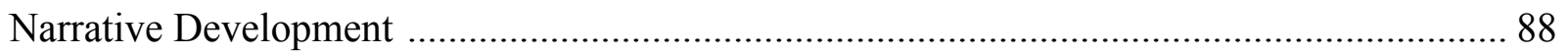

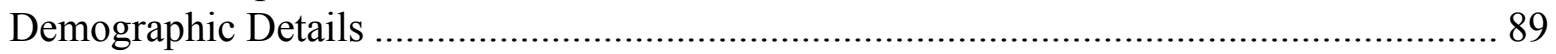

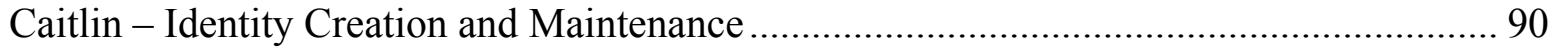

Danielle - Identity Creation and Maintenance ……….................................................... 95

Natalie - Identity Creation and Maintenance ………….............................................. 100

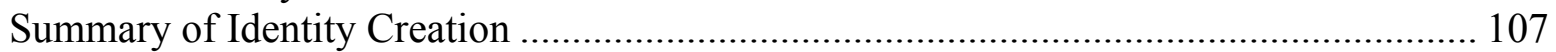

Social Capital and Beauty Influencers ……………......................................................... 108

Trust as a Form of Social Capital .......................................................................... 113

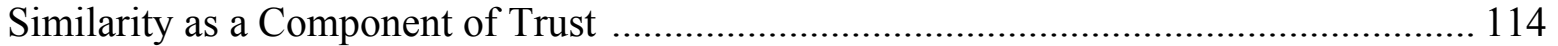

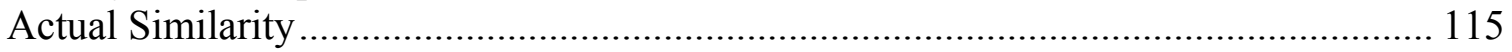

Perceived Similarity .............................................................................................. 118

Social Capital, Trust, and Similarity ........................................................................ 119

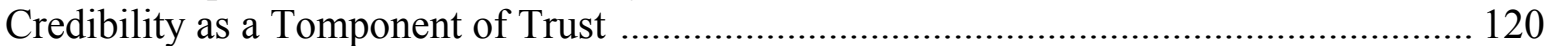

Credibility of the Influencer and Non-Sponsored Content ........................................... 124

Credibility of the Influencer and Affiliate/Free Product Content ................................. 125

Credibility of the Influencer and Sponsored Content .................................................. 127

Social Capital, Trust, and Credibility ………….................................................... 130

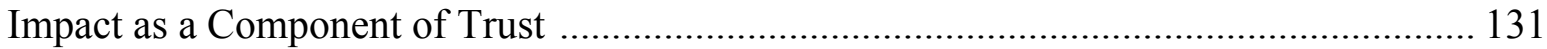

Non-Sponsored Content and Impact Statements ............................................................ 135

Affiliate/Free Product, Sponsored Content, and Impactful Comments ......................... 137

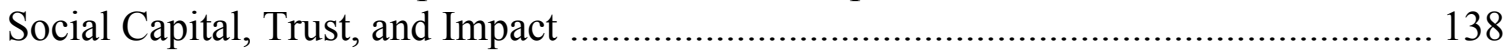

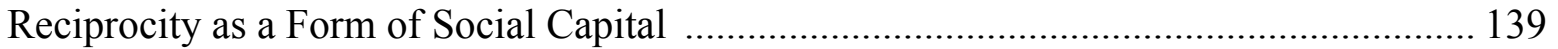

Involvement/Engagement as a Component of Reciprocity ............................................... 141

Involvement/Engagement: Projections by Influencers .................................................. 142

Social Capital, Reciprocity, and Involvement/Engagement ..................................... 145

Investment as a Component of Reciprocity ..................................................................... 146

Viewer Projections of Investment ........................................................................... 148

Investment, Reciprocity, and Social Capital............................................................ 153

Social Capital Maintenance - Redefining a Model ......................................................... 155

Authenticity and Beauty Influencers ............................................................................ 160

Influencer Initial Authenticity Categories on Real-Fake Matrix ....................................... 161

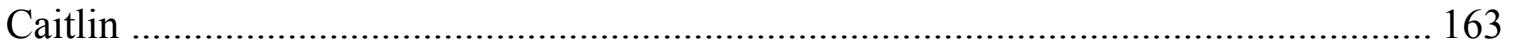

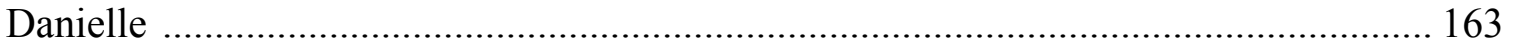

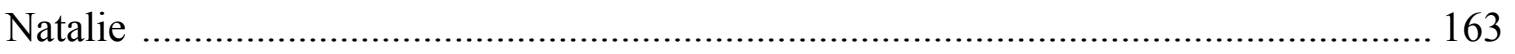

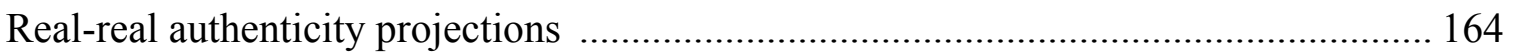

Deviations from Projected Authenticity ............................................................................. 165

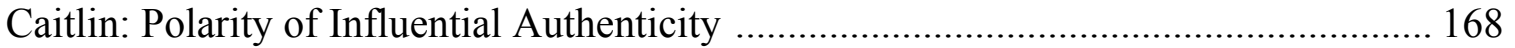

Danielle: Polarity of Influential Authenticity .......................................................... 171

Natalie: Polarity of Influential Authenticity ............................................................. 174

Fake-Real Authenticity in Practice .............................................................................. 176

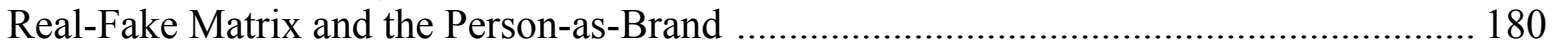

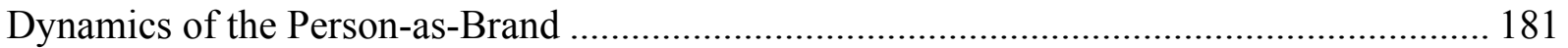

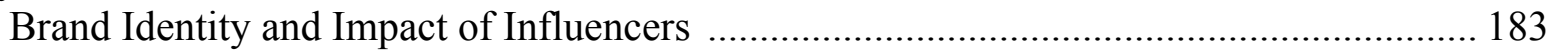




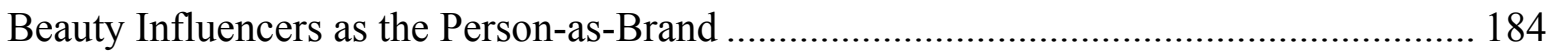

Non-Sponsored Content and Brand Identity Influence ................................................. 185

Affiliate/Free Product Content and Brand Identity Influence ..................................... 188

Sponsored Content and Brand Identity Influence ………….......................................... 192

Embedding the Person-as-Brand into the Brand as Person Identity Element ..................... 196

Reach of the Person-as-Brand in Relation to Brand Identity ............................................ 198

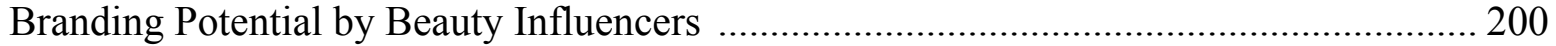

Message Reception through Digital Communication Channels ............................................ 202

Message Reception among Influencers and Viewers ................................................... 202

Non-Sponsored Content and Viewer Feedback ......................................................... 202

Affiliate Content and Viewer Feedback ...................................................................... 204

Sponsored Content and Viewer Feedback …………................................................ 207

Viewer Feedback and Impact on Beauty Influencers .................................................. 208

Influencer Monetization and Impact in the Digital Beauty Community ................................ 214

CHAPTER V: CONCLUSIONS _......................................................................................... 216

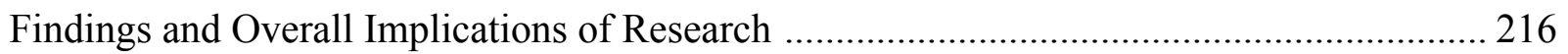

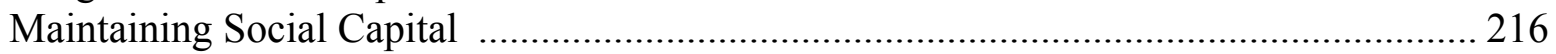

Authenticity - The Fake-Real Beauty Influencer .............................................................. 218

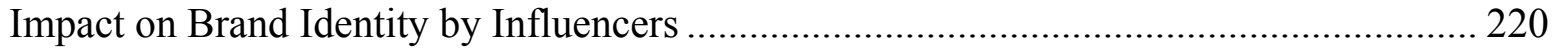

Message Reception and Positive Feedback Loop …………............................................... 221

Opportunities for Future Research and Constraints of Study ................................................ 222

Researcher Reflections ............................................................................................ 224

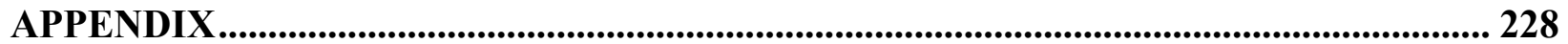

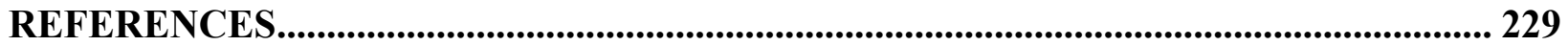




\section{LIST OF TABLES}

Table 1: Madden et al.'s (2012) classification scheme for video comments

Table 2: Demographic details of Caitlin, Danielle, and Natalie as projected through video content.

Table 3: Sponsored, affiliate/free product, and non-sponsored video statistics for Caitlin's beauty-related video content posted January - December 2016.

Table 4: Sponsored, affiliate/free product, and non-sponsored video statistics for Danielle's beauty-related video content posted January - December 2016.

Table 5: Sponsored, affiliate/free product, and non-sponsored video statistics for Natalie's beauty-related video content posted January - December 2016.

Table 6: Constructs of trust and reciprocity as a form of digital social capital, as adapted from Sander and Lee's (2014a) model

Table 7: Matrix of actual and perceived similarity statements in video content across three YouTube beauty influencers

Table 8: Matrix of credibility statements in non-sponsored, affiliate/free product, and sponsored video content across three YouTube beauty influencers.

Table 9: Matrix of impact statements in non-sponsored, affiliate/free product, and sponsored video content across three YouTube beauty influencers.

Table 10: Involvement/engagement statements in video content across three YouTube beauty influencers.

Table 11: Investment statements in video content across three YouTube beauty influencers... 147

Table 12: Uploading frequency, percentage of uploads in each category and uploads per week 


\section{LIST OF FIGURES}

Figure 1. Aaker's (1996) brand identity planning model....................................................... 33

Figure 2. Real-fake matrix as proposed by Gilmore and Pine (2007). ................................... 41

Figure 3. Reproduced model of elements of social network site capital as defined by Sander and

Lee (2014a)

Figure 4. Redefined model of digital social capital generation and maintenance for YouTube beauty influencers

Figure 5. Gilmore and Pine's (2007) polarities of influential authenticity, based on deviations from projected authenticity

Figure 6. Polarities of Caitlin's authenticity, as defined by deviations from projected real-real authenticity

Figure 7. Polarities of Danielle's authenticity, as defined by deviations from projected real-real

authenticity.....

Figure 8. Polarities of Danielle's authenticity, as defined by deviations from projected real-real

authenticity.... 174

Figure 9. Combined polarities of beauty influencer's authenticity, as defined by deviations from projected real-real authenticity, suggesting proper placement in a fake-real authenticity category

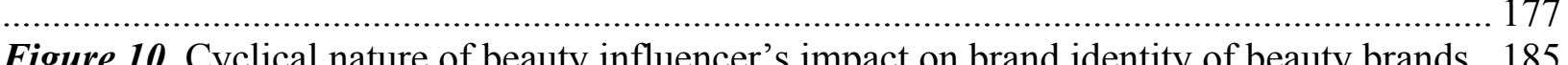

Figure 10. Cyclical nature of beauty influencer's impact on brand identity of beauty brands.. 185

Figure 11. Positive feedback loop generated by viewer-influencer communication practices in

YouTube beauty community. 


\section{CHAPTER I: INTRODUCTION}

"Hey guys! Welcome to my channel” proclaims an energetic beauty influencer on YouTube. Her bright red lips and perky personality are immediately apparent in her introduction, as she proceeds to tell her viewers, “Today's video is going to be a date night makeup tutorial, all drugstore. I'm actually using all Revlon products and I'm even using all affordable makeup brushes in this look, which is much requested for me on my channel." She continues to describe her look as having a soft eye with bold red lips as "it inspires confidence." She suggests having confidence is the key to a successful date. She takes a few moments to tell everyone the name of the lipstick she is wearing and promote the product's appearance, packaging, and smell. A white box displaying the name of the lipstick and brand imagery appeared. She opened the lipstick tube and smells it, saying "Ooh! I just got it on my nose!" This moment appeared to be candid, as if she should have edited it out of the video but chose to leave it as it showed a funny, real moment.

She takes a few seconds to ask her viewers to like the video and subscribe to her channel before "getting started" on the make-up tutorial. In the description box below the video it notes the video was posted in November 2016 and states, "Thanks to Revlon for partnering with me on this video to create an all drugstore makeup tutorial with no false lashes involved," followed by a statement again acknowledging "as requested" she has used drugstore makeup brushes. She signs off the statement with "XO" and her name. Comments by viewers below the video read, "You are always so fun to watch but also so informative!!!" and "Who else loves her videos?!" However, one viewer asks a question that stands out among the rest, "You mean 'Revlon makeup tutorial'?" As one viewer acknowledges, the video was created as a promotional piece for Revlon, showing their new lipsticks and how their products can be used to fashion a datenight appropriate look. The content was created specifically for the company, as a type of 
influencer-brand relationship. However, the majority of the comments by viewers do not question the authenticity of the positive appraisal of the products, instead compliment the beauty influencer for creating a beautiful look. There is an apparent bond between viewer and influencer, which seems to mask the notion these products are being promoted for personal profit.

\section{Gap in Knowledge - Identifying a Problem}

In the modern technologically-driven world, brands migrate to social network sites as a means of connecting with consumers. A recent report found $82 \%$ of businesses stated they were either fully integrated, or in the process of integrating, digital components of their brand (Terpening, Li, \& Akhtar, 2015). Additionally, 38\% of businesses planned to spend more than $20 \%$ of their advertising budgets on social media channels (Terpening et al., 2015). Fournier and Avery (2011) suggested brands that were active, interesting, humble, unprofessional, and honest received the most positive feedback on social network sites. It is important to note this definition of unprofessional brands did not imply being rude or disrespectful. Rather it suggested brands create sites which looked like an individual's and operated them as if they are a person, not a corporate entity paid thousands of dollars to build the 'perfect' site (Fournier \& Avery, 2011).

Researchers have reviewed how brands migrated to social media channels successfully (Kaplan \& Haenlein, 2010; Kietzmann, Hermkens, McCarthy, \& Silvestre, 2011; Mangold \& Faulds, 2009), as well as how brands built perceived authenticity on these sites (Fournier \& Avery, 2011; Pronschinske, Groza, \& Walker, 2012). In addition, research found that digital influencers played a key role in promoting brands on the Internet as they shared a common bond with members of a digital community (Spencer, Harding, \& Sheahan, 2014). A digital influencer is an individual on the Internet who has gained a large following of viewers and heightened 
digital presence through digital means. Influencers gained their followings through the affordances of the digital networks, such as being able to speak directly to viewers or connect with them through emotional appeals, beyond geographic boundaries.

The platforms in which digital influencers thrive are based on interaction and connectivity, where digital forms and practices create a culture in which resources are sought and shared among members (Caliandro, 2016). Bourdieu (1985) labeled this exchange social capital, which he defined as "the aggregate of the actual or potential resources which are linked to possession of a durable network of more or less institutionalized relationships of mutual acquaintance or recognition" (p. 248). These influencers share a level of social capital with the user, as they invite them into their lives and share with them aspects of their digital self.

These bonds were not generated solely through the affordances of a single digital platform. They were built and maintained, similar to face-to-face interactions, whereby an influencer and the viewers in her community engaged across the digital community, sharing scarce resources and fostering relationships. However, the building and maintenance of social capital in this context required a construct from which viewers could assess the truthfulness and reciprocity of an influencer, parallel to how individuals use non-verbal communication and body language to better define offline bonds (Putnam, 2000).

Gilmore and Pine (2007) suggested it is the projection of an authentic image which drew these communities together. Goldman and Kernis (2002) defined authenticity as the unimpeded operation of one's true self in the context of one's daily enterprises. In a mediated context, this assumption of the true self has been found to be related to portrayals of trust and reciprocity, similar to social capital (Baym, 2010; Livingstone, 2008). If an influencer was true to herself and 
did what she says she would, her maintenance of those projections allowed her to situate herself as being authentic in the community, capable of generating trust and reciprocity from viewers.

As digital influencers continued to project social capital and authenticity in the community, they began to use their leverage to grow the community. Influencers were compelled to invest more time and energy into videos, if the viewers reciprocated their actions by subscribing to their channels and increasing their video revenue. They also were able to establish trust among viewers as a function of time, where their consistent advice and credibility was justified through their status as a successful influencer. Influencers grew their communities to monumental heights, garnering millions of subscribers to their channels, allowing their videos to reach far beyond the boundaries of a single node of influence.

As YouTube was one of the first social network platforms to offer revenue for producing videos (Freeman \& Chapman, 2007), if an influencer noticed success in her community, there was additional external motivation to continue to produce content as a means of financial gain. Additionally, influencer notoriety drew interest from brands, as they wished to access the niche markets these influencers situated themselves within (Tolson, 2010). Through influencergenerated branding, companies were able to place products through these individuals (Booth \& Matic, 2011). As brands realized social network sites made it difficult for them to control the information being discussed and presented among consumers, they looked to influencers as powerful generators of impact within digital communities, as they were able to leverage their perceived social capital and authenticity with their viewers. This monetization through branding activities, predominantly the creation of sponsored video content, created an interesting paradox for influencers as they must sell a product to viewers through their connection to them. The communities which were built on social capital and authenticity now became venues for 
influencers to engage in branding activities, often in direct opposition to how the communities were formed. These channels were seen as being platforms of connectivity and interaction, not platforms of branding and sales promotion.

While research has suggested these digital influencers are able to affect consumer perception of brands through their status (Abidin \& Ots, 2015; Gillin 2007), it has yet to be explored how this managing of social capital and projection of authenticity are portrayed through monetization practices on YouTube, such as those offered in creating sponsored video content. As digital influencers continue to utilize brand sponsorships as a means of generating personal revenue, there is a gap in knowledge regarding how their social capital and authenticity projections may be modified or challenged through commoditization of content. The leveraging of social capital and authenticity toward financial gain may very well raise concerns among the individuals who crafted their digital influence, namely, the viewers.

\section{Purpose Statement}

In this dissertation, I explored how the creation and maintenance of authenticity and social capital by digital beauty influencers on YouTube were impacted by monetization. I also explored how branding principles, primarily through sponsorships, were affected by influencer involvement as well as how the digital community reacted to these constructs. YouTube is one of the few social network sites to offer direct revenue from generating content, primarily through advertising partnerships (Freeman \& Chapman, 2007). As digital influencers gained popularity, they were able to monetize their content both directly from the site and also through brandsponsored content (YouTube, 2016c). Yet, research has not explored how authenticity and social capital may be affected by these activities. Through this dissertation I examined how beauty influencers craft and maintain social capital and authenticity in conjunction with monetization 
activities, as a means of assessing the impact commoditization of content had on digital influence and feedback within the community.

Research questions. The main research question I assessed was how YouTube beauty influencers maintained social capital and projected their authenticity while engaged in monetization activities. The main research question asked was: How do YouTube beauty influencers maintain social capital and project authenticity while engaging in monetization activities?

Beauty influencers built their personal brands on the notion social capital is exchanged based on perceptions of influence and communication between entities (Burke, Kraut, \& Marlow, 2011). While individuals initially may have sought out pure information gain from the influencer, a form of bridging social capital, the connection to his or her authenticity and influence can cause the viewer to feel emotionally connected to the influencer over time, generating bonding social capital. This movement across a continuum from bridging to bonding social capital allows the influencer to remain both a source of information and emotional support, fostering deeper relationships and stronger connections (Valenzuela, Park, \& Kee, 2009). However, this perceived social capital could be damaged by both a lack of information and emotional attachment. If the influencer did not continue to provide quality information and emotional resources, there could be a loss in social capital between the content generator and viewer, lowering the generators overall social influence. Thus, my first sub-question addressed was: What practices are employed by YouTube beauty influencers to construct and maintain perceived social capital?

In addressing the bonds in a digital community, it was important to understand the practices beauty influencers use to construct authenticity, as Gilmore and Pine (2007) suggested 
changing between real and fake identity constructs could cause a disconnect between the influencer and viewers. The projection of authenticity is assessed subjectively, however, it should mirror a truthfulness to the influencers image and identity. As beauty influencers engaged in various types of sponsored and non-sponsored activities for financial gain, changes to authenticity constructs based on content could cause the influencer to loose authentic standing with the viewer. To address this, the second sub-question I posed in this research was: What practices are employed by YouTube beauty influencers to construct and maintain perceived authenticity?

Next, addressing the overall reaction of public members to sponsored content was important to understanding how influence functions as an extension of the brand (Aaker, 1996; Aaker, 1997). The overall impact of influencer-brand relationships was assessed as it was perceived by the viewer, through reactions to various types of sponsored content on YouTube. If the sponsorships are not producing favorable results among the viewers, who ultimately may become the consumer of the brand, the influencer may see additional losses in financial and social revenue. My third sub-question I sought to address was: How do YouTube beauty community viewers respond to monetization activities through the comments section offered by YouTube?

As this research questioned how a beauty influencer functioned as a member of a digital community, through sending messages to a receiver, both the role of the sender (influencer) and receiver (viewers) was assessed. It was important to understand what practices were utilized to construct and maintain authenticity and social capital through the lens of the message receiver, because if these concepts were not being communicated properly, the content generator could lose influence and status with viewers, thereby lessening her financial and social gains. The final 
sub-question asked was: How do YouTube beauty community viewers respond to and interact with beauty influencers' social capital and authenticity projections through the comments section offered by YouTube? Through these questions, this research generated knowledge regarding what practices were utilized to construct and maintain social capital and authenticity through varying levels of monetization activities as a means of assessing how these aspects shifted with the focus of content.

\section{Paradigm and Methodology Overview}

I assumed the stance that multiple realities exist, are socially constructed, and the linkage of investigator and object of study provide meaning (Sale, Lohfeld, \& Brazil, 2002; Tashakkori \& Teddlie, 1998). My epistemological views aligned with a constructivist paradigm, which suggested knowledge was bound in value and within the context the object of study created. Operating under this constructivist paradigm, I aimed to explore a digital culture as it was crafted and maintained in a specific time and context. As I explored how YouTube beauty influencers constructed realities through authenticity and social capital, a digital ethnography allowed for the exploration of culture through focusing on how the digital environment was utilized in shaping behaviors, interactions, and cultural processes (Ardévol \& Gómez-Cruz, 2014; Murthy, 2008).

A qualitative approach to research allowed me to “... seek answers to questions that stress how social experience is created and given meaning" (Denzin \& Lincoln, 2005, p. 10). As time and space are contextually bound in qualitative research, Creswell (2009) proposed researchers locate their data in everyday life, with the researcher having a central role in the research process, as the primary research instrument for data collection. In this study, my constructivist paradigmatic assumptions served to provide a shared level of meaning between the subject and phenomena, placing emphasis on time and context (Creswell, 2009; Denizen \& Lincoln, 2005). 
As defined by Caliandro (2016), digital ethnography studies the cultural connections of a networked society, where behaviors and interactions of the viewer's work to construct a digital context around shared practices. Digital ethnography puts the power of participant observation back in the hands of the participants, conveying the real-time richness of their lives, experiences, and environments (Masten \& Plowman, 2003; Varis, 2016). As such, a digital ethnography approach allowed me to take into account the nature of both the digital social environment and the affordances of the sites, as they both worked to create a space in which culture was generated and grown (Rogers, 2013).

An ethnographic content analysis of three YouTube beauty influencers served as the method for this research. The three influencers were selected based on pre-determined parameters from the literature and the nature of the research questions being addressed. Fifteen videos were selected for review from three influencers (five sponsored, five affiliate, and five non-sponsored) based on a set of established parameters, as a means of assessing how authenticity and social capital were projected across various sponsorship types.

$\mathrm{Wu}$ (2016) noted brands could fall into three categories of sponsored brand activities: (1) explicit sponsorship by a company where the influencer is paid to market a specific brand or product; (2) affiliations where influencers are paid when products are purchased from a brand or retailer through their a provided web link; and (3) free product sampling where influencers are sent products by a brand with the hope they feature the products in a video, however, there are no formal requirements for exposure of the product by the influencer. For the nature of this study, affiliate and free product videos were included together as they both provided economic capital to the influencer through a product, without direct payment by a brand. While influencers are not paid to mention content in this category, they were receiving a tangible gain through their 
relationships with brands in the affiliate/free product category. A third construct for this study was the inclusion of non-branded content, in which the influencer created video content that did not have any direct brand sponsorships or alignments. This non-branded content served as a point by which the impact of sponsored and affiliate/free product videos were compared and contrasted, to determine if monetization had an impact on the constructs in this study.

Gilmore and Pine's (2007) real-fake matrix, Sander and Lee's (2014a) digital social capital constructs, and Madden, Ruthven, and McMenemy's (2012) classification scheme for video comments served as the guiding principles for assessing authenticity, social capital, and message reception across the three beauty influencers. These three models were chosen for their relevance to this data type and integration of digital processes. Data was coded inductively using NVivo qualitative data analysis software, Microsoft Word files, and Excel spreadsheets. Open coding served as the basis for a framework analysis upon which matrices were developed for social capital and figures were developed for authenticity over the three sponsorship types. The framework analysis of textual comments also produced two figures in regard to branding mechanisms and message reception. This selection of methods and analysis best served the research questions by providing a context from which the constructs of this study were assessed across both the influencer and members of the digital community.

\section{Significance of Study}

Influencers who gained digital popularity through their authenticity and perceived social capital with the viewer appear to be able to benefit financially from their success. This opportunity has provided many digital influencers with the possibility of making YouTube a career. As influencers continue to gain popularity, especially those in the beauty community, brands utilize their status with the consumer as a means to gain access to a wide demographic 
market through sponsored video content (Booth \& Matic, 2011). However, it is unknown how this influence of financial gain impacts authenticity and social capital of the digital influencer.

As Gilmore and Pine (2007) suggest, authenticity is a subjective construct. However, the fluctuating of authenticity between real and fake tactics based on sponsorship type could cause viewers to assess certain types of communication as being inauthentic. If authenticity fluctuates too drastically, questions of intent arise among the message receivers. Additionally, social capital on YouTube typically is based on levels of information sharing and directed communication, generating and maintaining social capital as relationships progress (Burke et al., 2011). For instance, viewers initially may be drawn to influencer content to solve a problem through basic information gain, such as finding the right foundation for dry skin or learning how to apply lipstick properly. This social capital derived through information gain is referred to as bridging capital (Putnam, 2000). Over time, if the viewer deems the information gain directed to her needs, she may begin to interact more closely with the community, communicating with the influencer through the comments or offering support to fellow members. As the group begins to homogenize based on their shared needs and information being presented, the social capital switches to a bonded form, where members are more interested in sharing emotional resources than gaining information (Putnam, 2000). They care about the well-being of the community and look to the influencer as the piece which brings them all together.

As this digital social capital is rooted in trust and reciprocity, the information shared and emotional resources projected through varying types of sponsored and non-sponsored content could impact perceived connection by the viewer (Valenzuela et al., 2009). If viewers feel the information being provided is skewed based on sponsorships or if they feel the content no longer has an emotional connection, the perceived social capital between the influencer and viewer 
could be affected negatively. As both influencers and brands continue to rely on consumergenerated advertising as a means of creating revenue, it is integral that viewers continue to put trust into beauty influencers and remain influenced by their content. If authenticity and social capital are questioned, there could be negative repercussions for all parties involved. Thus, this research sought to explore how these constructs were expressed through YouTube beauty influencers and viewed by members of the digital beauty community as a means of assessing how digital influence was affected by monetization activities.

\section{Reflexive Research Assumptions}

There were a few key assumptions I held which influenced the development of the research agenda. As Creswell (2009) suggested, reflexivity of the researcher in regard to assumptions should be addressed as a way of realizing the subjectivity in qualitative research.

The first assumption held was that the recent success of beauty influencers on YouTube have prompted businesses to utilize their status as a means of promoting products. This assumption was furthered by research suggesting brands look to influencers as key components in brand identity building, as they have the ability to reach large quantities of niche markets (Booth \& Matic, 2011; Marwick, 2013). While the interests of these markets may be narrow, such as the perceived interest in cosmetic products in the beauty community, these influencers are able to directly reach the consumers who have the highest amount of vested interest in these products. Through this content, brands look to attach their products to influencers with whom viewers find similarities to their personal self-concept, thereby increasing feelings of affinity through similarity (Abidin \& Ots, 2015; Gillin, 2007; Hong \& Zinkhan, 1987; Krampf \& Ueltschy, 1997). 
The second assumption was YouTube has created a platform upon which beauty influencers are able to project their message to a wide, globalized audience. As of December 2016, there are over 6,500,000 global YouTube channels promoting beauty in some format. A recent article by Mosendz and Bhasin (2016) suggests YouTube is "helping to sell a lot of makeup" (n.p.) through the innovation of top beauty influencers. Their messages are reaching global communities and creating platforms of powerful advertising success.

The final assumption is that viewers formed a bond with beauty influencers on YouTube. Tolson (2010) found consumers identify more with individuals on YouTube or other social media influencers, as they present an air of transparency. Viewers may gain scarce resources through interacting with influencers in the digital community, which is prompted by the need for information gain or emotional support (Putnam, 2000). This assessment, however, also may be guided by the viewers' appraisal of influencer authenticity, as digital influencers built their communities on the notion that they were real individuals, part of a digital culture capable of generating authentic relationships and communication (Caliandro, 2016; Gilmore \& Pine, 2007). As constructs of affinity often are subjective in nature, it was assumed that concepts such as social capital and authenticity served as the basis for developing relationships in a mediated context (Booth \& Matic, 2011; Burke et al., 2011; Valenzuela et al., 2009).

\section{Summary of Chapters}

This chapter has outlined a gap in the literature regarding authenticity and social capital of digital beauty influencers, which was addressed through a qualitative research methodology. This chapter also outlined the significance of this study, as well as the reflexive research assumptions. 
Chapter two provides a review of literature regarding social network sites, social capital, branding, authenticity, and the incorporation of digital influencers into branding activities. The chapter defines social capital and authenticity, as well as reviews the evolution of branding on social network sites. The chapter closes with a discussion of how digital influencers are utilizing sponsored activities as a form of financial advancement, including debates surrounding how authenticity and social capital may be projected through these activities.

Chapter three is a review of the methodology utilized in this research. The chapter opens with a discussion of paradigmatic assumptions of the research, which is followed by the rationale for selecting a qualitative methodology. A review of the digital ethnography theoretical framework provides a context for which culture may be assessed in a mediated environment. A discussion of ethnographic content analysis and framework analysis, provide the defining features of the method and data analysis technique utilized for this research. The chapter discusses the parameters leading to the selection of the three YouTube beauty influencers for this study, as well as how the varying levels of video sponsorship content were selected. The chapter closes with a discussion of tactics for assessing rigor in qualitative research and ethical considerations.

Chapter four provides the thematic interpretation of the results for this study. This chapter opens with the building of digital identities for each influencer, through her own projections as well as my assessments made based on my position as a viewer in the YouTube beauty community. The chapter then addresses each construct of this study (social capital, authenticity, brand identity management, and communicative practices) through the projections of the influencer. Included in each section is a discussion of viewer response to influencer projections, as a point of triangulation, in addition to providing a context from which message reception is 
assessed. The chapter closes with a discussion of how these four constructs work in unison within a digital community, specifically in regard to the monetization of YouTube content.

Chapter five serves as the conclusion for this research. This chapter will offer a recapitulation of the results and findings drawn from the research. Included will be a discussion of each construct, including how they relate to prior research, as well as the new findings presented by this study. This chapter will conclude with a discussion of opportunities for future research based on limitations of the parameters for this study, in addition to a discussion of reflexivity in this qualitative research. 


\section{CHAPTER II: LITERATURE REVIEW}

This chapter offers a comprehensive examination of social capital concepts, authenticity, and branding practices as related to this study. An overview of the digital web, social network sites, branding practices, and digitization of the brand provide a context for the nature of the research being presented. Through defining social capital and authenticity, both offline and online, the content discussed provides a background on relevant theories, definitions, and concepts as they relate to the present research. Relevant information regarding social network sites integration into society, digital branding, consumer-generated advertising, and digital influencers, as they relate to online content creators, is offered for additional context.

\section{Technological Change - The Introduction of the Web}

Throughout history, technological change has provided a framework by which a society may adopt and grow. Technological advances are neither sudden nor are they discontinuous, but are related to society at the time they are introduced and in the ways upon which they are utilized (Cowan, 1997). Parasuraman (2000) suggested this likely is due to technology readiness, a concept that the embracing of new technologies relies on innovation of the medium, as well as perceived consumer optimism, discomfort, and insecurity with it. For instance, critics of the telephone cited it "weakened character, made people lazy, broke up home life, and reduced visiting among friends" (Fischer, 1992, p. 261). While the technology was innovative, it was not considered useful at the time, even condemned for its contribution to the "failure" of society. New technologies present unique innovations, however, they also often require time to determine what the individual gains in utilizing the technology, as well as its impact on society at large. The extent to which the consumer of a technology embraces the curve is a pinnacle in understanding technology readiness (Parasuraman, 2000). It is the combination of these many 
societal and individual facets regarding technological advances that provided a timeline for mass acceptance (Morris \& Ogan, 1996).

Perhaps one of the most prolific technologies introduced in recent decades was the World Wide Web by Tim Burners-Lee in 1989 (Getting, 2007). This version of the Internet was termed Web 1.0 and defined the web as being read-only (Cormode \& Krishnamurthy, 2008; Getting, 2007). In this version of the web, users were viewed as passive consumers of information, as most websites focused on distributing information to the consumer in a static form (Baym, 2010; Aghaei, Nematbakhsh, \& Farsani, 2012). This web had very little user interaction or content contribution, as it focused on the user as a consumer of information. As more individuals migrated to the Web 1.0 system in the early 2000s, users began exploring new opportunities provided by these sites. Society was ready to gain control over the content they consumed and quickly adopted the Internet as a way to break information barriers (Getting, 2007).

Web 2.0 was defined by O'Reilly (2005) as being a platform-centered version of the web, where users now are able to both consume and produce content. In this form of the Internet, users are encouraged to generate new content and distribute it on a global scale (Baym, 2010; Kaplan \& Haenlein, 2010). There no longer is a need for formal training to create new online content, but rather a simple desire to connect. With the click of a button, a user can broadcast information across the globe. It is the technology of Web 2.0 which has allowed a shift from user-as-consumer to user-as-producer (Baym, 2010; Jenkins, Purushotma, Weigel, Clinton, \& Robison, 2006). Many consider the introduction of the social network site as the first communication platform of Web 2.0 to provide users control over the content they produced (Baym, 2010; boyd \& Ellison, 2007; Kaplan \& Haenlein, 2010). 
It was boyd and Ellison (2007) who produced one of the first definitions of a social network site. The researchers defined these sites as being web-based production platforms which allowed a user to: (1) construct a profile, either public or semi-public, within a defined system, (2) develop a list of fellow users with which they share some connection, and (3) navigate shared connections between his or herself and other users of the system (boyd \& Ellison, 2007). Social network sites were the first platforms to provide the user a new level of control over the content they generated and viewed. These sites extended beyond physical boundaries, breaking down centuries-old barriers to communication. Users were able to engage with others through these sites, by generating content, building relationships, and gaining new control over their communication.

As Web 2.0 continued to evolve, users realized the Internet provided them with new opportunities, namely, a shift from technology as utility to customization (van Dijck, 2013). With the affordances provided by Web 2.0, the user was able to become a producer of content, to customize the online experience in a way never before realized. The introduction of Myspace (2003), Facebook (2004), YouTube (2005), and Twitter (2006) offered variations of the social platforms necessary for users to produce this content (van Dijck, 2013).

However, it was the quick adoption of social media platforms and their affordances which made these sites unique. In 2007, it was estimated $6 \%$ of the world's Internet population over age 15 logged onto a social network site; by 2011, just four years later, the number jumped to $82 \%$ (van Dijck, 2013). Facebook, created in 2004, was one of the first social network sites and is still the most globally recognized social media platform (Ellison, Steinfield, \& Lampe, 2007). In April 2016, Facebook revealed it had amassed 1.09 billion daily active users (Protalinski, 2016). In June, Twitter (2016) reported over 313 million monthly active users, with 
$82 \%$ using their mobile platform and $79 \%$ of the accounts being from outside the United States. Instagram (2015), a photo-based social network site, reported over 400 million users in September 2015. Kaplan and Haenlein (2009) found that in 2009 the social network site YouTube saw uploads of nearly 10 hours' worth of video content every minute to the platform. YouTube (2016a) announced they have over one billion active viewers as of 2016, who watched hundreds of millions of hours on YouTube daily. The site also offers local versions in over 88 countries and is able to translate videos in 76 different languages (YouTube, 2016b). A unique affordance of YouTube is it was one of the first social network sites to offer not only a platform of communication to influencers but also tangible resources, in the form of direct revenue (Freeman \& Chapman, 2007). The revenue may be gained through a variety of metrics on the site, from subscription totals to video views to name a few, prompting influencers to grow their communities larger and more diverse in an effort to create more connections, equating to more potential for personal gain.

Top earning content generators on the site tout millions of active subscribers, with video views reaching over 10 million in just months. Jenna Mourey-a comedian who began her career on YouTube as Jenna Marbles over six years ago — currently has 16.5 million subscribers to her channel, with a recent video "Ultimate 100 Coats of Things Video" reaching over 10 million views in just two months (Mourey, 2016). The far-reaching scope and communicative abilities of social network sites provided opportunities for content to become limitless, where the free generation of content, perceived social influence and trust open the channels for a flow of information and resources.

Affordances of social network sites. Some researchers suggested the mass acceptance of social network sites was due to their creative and communicative abilities (Baym, 2010; Ellison 
et al., 2007). Research suggested a reason for the adoption of these sites was the user becoming a producer of the content they desire, shifting from passive consumer to active participant in the digital culture (Constantinides, Lorenzo-Romero, \& Gomez-Boria, 2009; Cova \& White, 2010; Jenkins et al., 2006; O'Reilly, 2005). This user-generated content (UGC) is built on the fundamental principle that users will distribute content across a Web 2.0 platform, typically a social network site, as they provide the greatest affordances for global communication (Kaplan \& Haenlein, 2010). This allows users the possibility of expressing their creativity and distributing content to any interested parties (Jenkins et al., 2006; Labrecque, Markos, \& Milne, 2011; van Dijck, 2009).

In this form, intent is of little concern, as users may be unaware they are creating content and distributing it. For instance, when posting a Facebook status or uploading an Instagram photo, often considered mundane digital activities in terms of scale and reach, the user is still creating unique content which made his or her presence known in an online context (boyd \& Ellison, 2007; Ellison, Steinfield, \& Lampe, 2007; Kaplan \& Haenlein, 2010). The desire to create content is not a new ideology, as creators have existed for generations; however, social network sites offered individuals the opportunity for digital creation, transcending geographic barriers.

In addition to creating content, social network sites also allowed users to craft a digital version of the self. This is a complex process which begins by defining the perceived versus desired self and how congruence between the two may be reached (Labrecque, Markos, \& Milne, 2011). While creation of the self is achieved in a face-to-face interaction (Goffman, 1963), research suggested many users preferred to define themselves in a digital context as well, through the use of social network sites (Baym, 2010; Ellison et al., 2007). As consumers look to 
define their personal self, the affordances of online platforms provided unique tools to make certain self-identification techniques much more efficient (Labrecque, Markos, \& Milne, 2011). Users were able to navigate to sites and share information quickly, thereby helping to build a desired identity. The user could upload images and text that shape his or her identity, in addition to connecting with friends who help to define his or her community of support (Ellison et al., 2007). These sites offer the possibility of crafting a self both actual and ideal, through the careful selection of disclosed information across a network. In defining a digital self, the user is able to establish presence in a digital culture and work to gain resources from the connections shared within these sites.

\section{Defining Social Capital}

This study utilized Bourdieu's (1985) definition of social capital as the acquisition of shared resources through the interactions of a members in a network as the basis for which social capital will be assessed. The social networks in which Bourdieu's (1985) definition existed are not considered a natural phenomenon, as they were constructed through various investment strategies in which benefits were derived from group membership and shared reciprocity (Portes, 2000). These networks were built through exchange of information, norms, and trust from which coordination and cooperation were the basis of establishing mutual benefit for sharing resources (Bourdieu, 1985; Putnam, 1995). The sharing of social capital led to a variety of resources, such as: economic (investment tips, loan agreements, or market sharing); credibility in a society; capacity to organize groups; and increases in cultural resources, which situated oneself as being integral to cultural stability and influence (Paxton, 1999; Portes, 2000). Each of these social resources provided a benefit to the receiver, however, they also establish a bond with the sender, from which future reciprocal benefits could be derived. 
Putnam (2000) further defined the notion of connection between individuals as being a predictor of social capital through the concepts of bridging and bonding capital. Granovetter (1973) described the apparent strength of a bond as being a "combination of the amount of time, the emotional intensity, the intimacy (mutual confiding), and the reciprocal services which characterize the tie" (p. 1361). Granovetter (1973) posited strong ties were those which have high levels of these characteristics, creating the strongest bonds, however, also requiring time to construct trusting relationships. Weak ties were those between individuals who see connections as a source of "monetary" exchange, where there was a perceived benefit for both parties without the need for sustained connection (Granovetter, 1973).

From this discussion of strong and weak ties, Putnam (2000) delineated two basic forms for social capital: bonding and bridging. Bridging social capital was derived from weak ties (Granovetter, 1973), through connections with casual acquaintances (Putnman, 2000). Bridging social capital is of importance as it leads to tangible outcomes such as novel information and broad views of the world, stemming from the sharing of information across individuals with diverse backgrounds (Ellison, Steinfield, \& Lampe, 2011). Bonding social capital reflects connections with strong ties (Granovetter, 1973), typically family and close friends, who are in a position to provide emotional support or access to more scarce resources (Ellison et al., 2007). Bonding social capital provides individuals in a network with more abstract resources such as emotional support, affection, and trust (Kavanaugh, Reese, Carroll, \& Rosson, 2005).

While the two forms of social capital are formed and maintained in differing ways, Putnam (2000) noted both are important for sustaining healthy networks. Bridging social capital allows connections between heterogeneous groups, facilitating the exchange of ideas among members (Kavanaugh et al., 2005). Bridging social capital is focused on providing unique 
information, rather than support or emotional exchanges, as is typical in bonding social capital (Kavanaugh et al., 2005; Putnam, 2000; Wellman, 2001). However, it is bonding social capital which keep community groups viable, through voluntary investments of time and mutuality, providing scarce emotional resources (Kavanaugh et al., 2005; Putnam, 2000; Wellman, 2001). It is the careful maintenance of bridging capital for information resources and bonding capital for emotional resources that created the framework from which networks may become communities (Kavanaugh et al., 2005).

The notion of a networked community did not end in the built environment, as the introduction of social network sites expanded the opportunities for building and maintaining social capital across new boundaries (boyd and Ellison, 2007; Ellison et al., 2007; Ellison et al., 2011). Burke, Kraut, and Marlow (2011) proposed "the use of social network sites in particular have the potential to change the costs of communication, the number and character of people with whom one keeps in touch, and the nature of the communication one has with them" (p. 572). Through social network sites, individuals have the opportunity to interact with other users on both surface and deeper levels, crafting bridging and bonding capital. Yet, these two type of capital are not mutually exclusive on these sites, relying on certain types of communication and relationship building mechanisms to define their existence.

Burke et al. (2011) suggested there are two types of communication on social network sites: undirected and directed. Undirected communication is defined as passive consumption and generalized broadcasting, such as sharing information on the Facebook news feed or watching a YouTube video recommended from the home page (Burke, Kraut, \& Marlow, 2011). These undirected messages are novel in they are able to facilitate the building and maintenance of bridging social capital (Ellison et al., 2007; Ellison et al., 2011). Thus, interactions between users 
can be regarded as an information flow, where similarities in opinions or shared digital publics may prompt users to explore relationships with others (Burke et al., 2011; Kavanaugh et al., 2005). Additionally, the interconnectivity of social media allows users to share connections in a "friend-of-a-friend" manner, such as interacting with an individual through a shared mutual friend on Facebook. If an individual shares content that matches another user's interests, the shared connection can allow the message to be distributed farther than in face-to-face communication. In this sense, it is plausible that creating and consuming undirected messages can lead to the flow of information and the potential to increase bridging capital.

Directed communication provides an apparent link between forging bridging and bonding social capital. Burke, Kraut, and Marlow (2011) defined directed communication as personal, one-on-one exchanges. Social network sites offer the possibility for this exchange through content such as direct messaging, commenting capabilities, and synchronous chat features. However, "one-on-one exchanges" do not have to necessarily imply two single individuals communicating. On social network sites, the shared connectivity between users promoted feelings of affinity from one individual to entire communities of interest, as users seek out information and social interaction (Haridakis \& Hanson, 2009). Rubin (2002) found active users of social network sites develop affinity with the content they select to view. This affinity was displayed through purposive information search and greater interpersonal activity levels on the site, elements suggesting both undirected and directed communication, leading to bridging and bonding social capital (Haridakis \& Hanson, 2009; Rubin, 2002).

One suggestion for this purposed affinity is the presence of parasocial interactions (Horton \& Wohl, 1956; Labrecque, 2014; Levy, 1979; Rubin, Perse, \& Powell, 1985). Horton and Wohl (1956) defined the concept of parasocial interactions as being the development of 
relationships between consumers and mass media. Researchers further explored this concept, noting that consumers tended to engage in illusionary relationships with media personas, as if they were present and engaged in face-to-face communication (Levy, 1979; Rubin, Perse, \& Powell, 1985). This even may be displayed in daily interactions, such as talking about television characters or actors as if they are your personal friends, creating the illusion of a relationship with these individuals, even if there has never been direct contact with them. Labrecque (2014) suggests that this could be due to the notion that carefully constructed verbal and nonverbal mechanisms by media personas feel as though they are directed communication, capable of generating social capital between individuals, through the illusion of a bond being present between two entities, even if it did not physically exist.

While parasocial interaction research originally was framed in terms of television or film personas, the asynchronous nature of social network sites has created the opportunity for individuals to develop these same types of interactions (Horton \& Wohl, 1956; Labrecque, 2014). Influencers are able to access niche markets through their membership in the community and perceived notoriety (Tolson, 2010). Once they have elevated this status, they begin to function like media personas, projecting their social capital on a one-to-many level. There may be a single influencer attempting to connect with networks of over 2,000,000 subscribers. It is nearly impossible for that influencer to reach every viewer individually, thus, he or she must rely on the presence of parasocial interactions to form these perceived bonds.

As Perse and Rubin (1989) suggested, parasocial interactions can arise from a single occurrence. The cultivation of directed, impactful information and identity projections by the influencer can draw a viewer to his or her channel through one video. If the influencer is able to project information that is relevant to viewers, matching constructs of the viewer's own self- 
concept in an effort to bolster identity building and maintenance, there is the possibility that he or she will feel compelled to engage in a relationship with that influencer, in an effort to continue to gain social capital resources. In most instances there is no direct connection between the influencer and the viewer. The projection from the influencer is to the audience as a whole, carefully crafting messages and information in a way that feels directed toward those with similar interests and passions. If the viewer feels affinity with this content, there is a strong possibility that he or she will engage in a parasocial interaction with the influencer, through watching future videos, commenting on content, or even extended bonds beyond the confines of YouTube to other social network platforms. While there is no physical relationship that exists, the illusionary one is strong enough to produce the bonds necessary for the exchange of bridging and bonding social capital. The asynchronous communication affordances of social network sites allowed for these parasocial interactions to occur, ultimately creating new paths for the exchange of social capital on these sites.

Research on the generation and effects of social capital on social network sites produced contradictory results in regard to how the sites maintain capital (Ellison et al., 2007; Nie, 2001; Steinfield, Ellison, \& Lampe, 2008; Turkle, 2012). One side suggested social network sites are leading to the degradation of personal, cue-based communication through a decrease in face-toface communication opportunities (Nie, 2001; Turkle, 2012), while the other proposed social network sites lower barriers to participation and allow for bonds to be formed beyond global boundaries (Ellison et al., 2007; Steinfield et al., 2008). However, both sides agreed the positive and negative effects of social media use for bond formation are contingent upon the ways the user engages with the platform (Valenzuela, Park, \& Kee, 2009). 
Williams (2006) found the type of relationships and affordances offered on social network sites can predict the kind of social capital formed. In this sense, technology does not mold the social capital, but rather it is the actions of the user. Social network sites allow for broadcasting a variety of information, often between heterogeneous groups where information may not be freely shared due to geographic or cultural limitations (Valenzuela et al., 2009). This sharing of information leads to the formation of bridging capital where uses can mobilize information and share opportunities across spatial boundaries. In regard to bonding social capital, McQuail (2005) proposed social network sites may hold the key for growing strong bonds through social interaction. These sites allow individuals to craft a digital identity that share commonalities and directed communication across others with similar interests (Burke et al., 2011). As groups on these sites begin to homogenize, they lose some of the information sharing capabilities of bridging social capital, as the content being shared grows in similarity (Valenzuela et al., 2009). However, as the homogeneity increases, so do feelings of trust and reciprocity, where users feel connected to the community and foster social interaction among group members, thereby generating the emotional resources necessary for bonding capital (Burke et al., 2011; Valenzuela et al., 2009). There is a trade-off from information sharing in bridging capital to the emotional resources gained from bonding capital.

Sander and Lee (2014a) suggested digital social capital is the result of two actions: access and motivation. First, the individual must have access to a digital environment and gain membership in a group, to exchange information and garner bridging capital. Once saturation has been reached in terms of information exchange, motivation to gain additional resources - such as emotional bonds or affection - often are driven by the involvement, investment, and benefits gained from the group (Sander \& Lee, 2014a). Individuals who passively participate in these 
activities were less likely to achieve this deeper, bonding capital than those who actively participate, suggesting active members were most likely to benefit emotionally on social network sites (Burke et al., 2011; Valenzuela et al., 2009; Williams, 2006). The content on YouTube also is unique to other social media sites as it provides verbal, non-verbal, and visual cues in the form of asynchronous videos, not solely text-based disclosure which is easily changeable (Booth \& Matic, 2011; Marwick, 2013). Participation may be defined by new parameters on YouTube, as liking a video or commenting on the content is not a required component of interaction.

The defining features between bridging and bonding social capital are not as easily dichotomized in online contexts (Pelling \& High, 2005; Valenzuela et al., 2009). Through the sharing of information, bridging capital is able to be formed. However, as Burke et al. (2011) proposed, the directed communication availability in social network sites helps to build trust and reciprocity between individuals, by lowering the barriers to communication and relationship building. Communication may become even more directed through community development, as users grow more comfortable sharing information and building bonds, eventually leading to the sharing of emotional resources, such as encouraging messages or support for personal choices. Thus, social capital can shift focus in regard to the nature of the communication on a social network site, moving on a continuum from information sharing to emotional bonding. Individuals essentially are branding themselves, by creating a virtual image that portrays the information they have to offer and directs messages with whom they share a community, offering both bridging and bonding social capital (Labrecque, Markos, \& Milne, 2011). This personal branding has allowed individuals to maximize the affordances of social network sites, which opened the door for further defining various communicative capabilities and the evolution of social capital in a digital context. 


\section{Evolution of Branding}

Kotler (1997) defined a brand as a name, term, sign, or symbol intended to identify the goods or services of someone or something which is trying to promote to another. The American Marketing Association provided a similar definition, with the exception a brand is intended to use various names, terms, signs, symbols, or designs to differentiate a good or service from competitors (as cited in Wood, 2000). Branding could be both personal and service based, as individuals and companies brand themselves in similar ways. In the context of this study, branding implied any efforts to create a brand identity toward the commoditization of a good or service, either through individual efforts or corporations. For clarity, the following discussion of branding will assume branding as an act in the context of business relations, where any branding entities will be referred to as a company.

Branding often begins with a focus on the tangible characteristics of a product or service, in an effort to differentiate these items from competitors (Aaker, 1991; Holt, 1995), as the tangible characteristics provide physical qualities around which to build ideologies. The tangible elements of the brand focus on early definitions of branding, where the name, slogan, functional values, and performance of a brand is utilized as a means to differentiate the product or service from competitors (Aaker, 1992; de Chernatony \& Riley, 1997). Proctor and Gamble once used the slogan "Look, Mom - no cavities," inscribed over Norman Rockwell drawn artwork of smiling, happy children holding positive check-up reports from the dentist, to highlight the notion Crest toothpaste was the ultimate choice in oral hygiene care (AdvertisingAge, 2003). While this is certainly an ideal image of the brand, consumers actually believed the advertisement based on the legitimacy of the claims. In 1960 the American Dental Association recognized Crest as the first and only toothpaste to have an effective decay-preventing agent in 
its formula (AdvertisingAge, 2003). Following the report, Crest's stock jumped to $25 \%$ of the market share in 1961, as consumers believed the tangible product being offered by the brand, toothpaste, was indeed the best among the competition (AdvertisingAge, 2003).

This tangible assessment of brand claims did not end with toothpaste, as cosmetic companies still engage in creating products that offer claims backed by science. Many skin care brands proclaim their products to be able to reduce wrinkles, soften skin, and minimize fine lines. While these phrases are carefully crafted to produce impact in marketing, they are backed by scientific research. Cosmetic scientists utilize a variety of tools and techniques to test the claims of products, such as use of a cutometer, which creates a vacuum on a small patch of skin to measure elasticity when suction is released (Reisch, 2016). Statistics are used to back product claims thereby providing another layer of tangible evidence of a product's ability, suggesting to consumers it is superior to competitors based on these replicable results.

The Crest campaign and scientific testing of skin care products highlighted another critical component in the history of early branding, that of the role of the consumer in accepting these claims. Ambler (1992) suggested a brand is an entity that promises a bundle of attributes, from which someone will buy and receive satisfaction. This definition proposed while the tangible elements of a brand are important (Holt, 1995), the intangibles are equally relevant in how the consumer perceives the good or service and ultimately benefits from it (Ambler, 1992). Brands begin with the tangible elements to establish a reputation, building on the functional characteristics of the brand to add value to the product or service (de Chernatony \& Riley, 1997). The brand then is able to use this reputation to build relationships, increase confidence in the brand as a whole, and add value in a more symbolic fashion (de Chernatony \& Riley, 1997). 
The process of branding has evolved over the course of history, from the focus on tangibles and intangibles, to a vision of branding which goes beyond the surface definitions of physical and symbolic brand characteristics. Gardner and Levy (1955) established one of the first definitions of branding that went beyond the surface of the brand and called for a need to identify more in-depth purchasing motivations. They proposed a product had a social and psychological nature, in addition to the physical forms, and those feelings, ideas, and attitudes form the image of the brand to the consumer (Gardner \& Levy, 1955). It is the consumer perception of the brand, on a physical, psychological, symbolic, and social level that helps him or her to craft a holistic view of the brand (Burmann, Jost-Benz, \& Riley, 2008; Harris \& de Chernatony, 2001). The brand image is a way for the consumer to look at the brand and determine how it ultimately has impacted aspects of his or her life. While the final brand image is crafted in the mind of the consumer, companies may put forth certain attributes meant to guide him or her to an understanding of the brand, such as those meeting the functional, symbolic, and experiential needs of the consumer (Park, Jaworski, \& MacInnis, 1986).

The brand image, thus, is the overall impression in the consumer's mind regarding a brand formed through exposure to the various elements of the product or service being offered, and through determination of how they meet certain needs (Padgett \& Allen, 1997; Park et al., 1986). The brand image is constructed by the consumer, through analyzing and framing the associations they have with the brand. While content is put forth by the brand to help mold these associations, in this stage of branding, it is ultimately the consumer who decides what the final brand image will be through the subjective meanings imposed on the brand.

After spending some time focusing on how to create attributes which positively affected brand image, companies began to re-imagine the branding landscape. A shift in ideology 
regarding how a brand can be built prompted the emergence of a new concept - brand identity. While a brand image was still being formed by the consumer based on his or her perceptions of the brand, companies began to focus on creating an active identity, one that looked forward and promoted the company values (de Chernatony, 1999). A brand identity is meant to promote the constructed reality of the company, as a way to tell the consumers where the brand is going and why they should come along with them.

The identity should resonate with consumers and represent what the company will do for them over time, showing that a company is personable and looking at the long-term benefits their products or services are meant to offer (Aaker \& Joachimsthaler, 2000; de Chernatony, 1999; Kapferer, 2008). Consumers make an investment in brands to which they feel a connection, with a solid brand identity personalizing investment (Gilbreath, 2010). A brand with a strong identity can create a trusting bond between consumers and retailers, just as a human friendship functions, fostering long-term relationships between the two entities (Burmann et al., 2009).

Branding- extended identity measures. As branding continued to evolve with consumer perceptions and needs, Aaker (1996) proposed a model of brand identity which drastically shifted the focus of sales-based marketing to a more strategic plan. Aaker's (1996) model, as seen in Figure 1, proposed brands could build a strong identity through innovation, by identifying patterns to clarify, enrich, and differentiate their brand through various perspectives. 


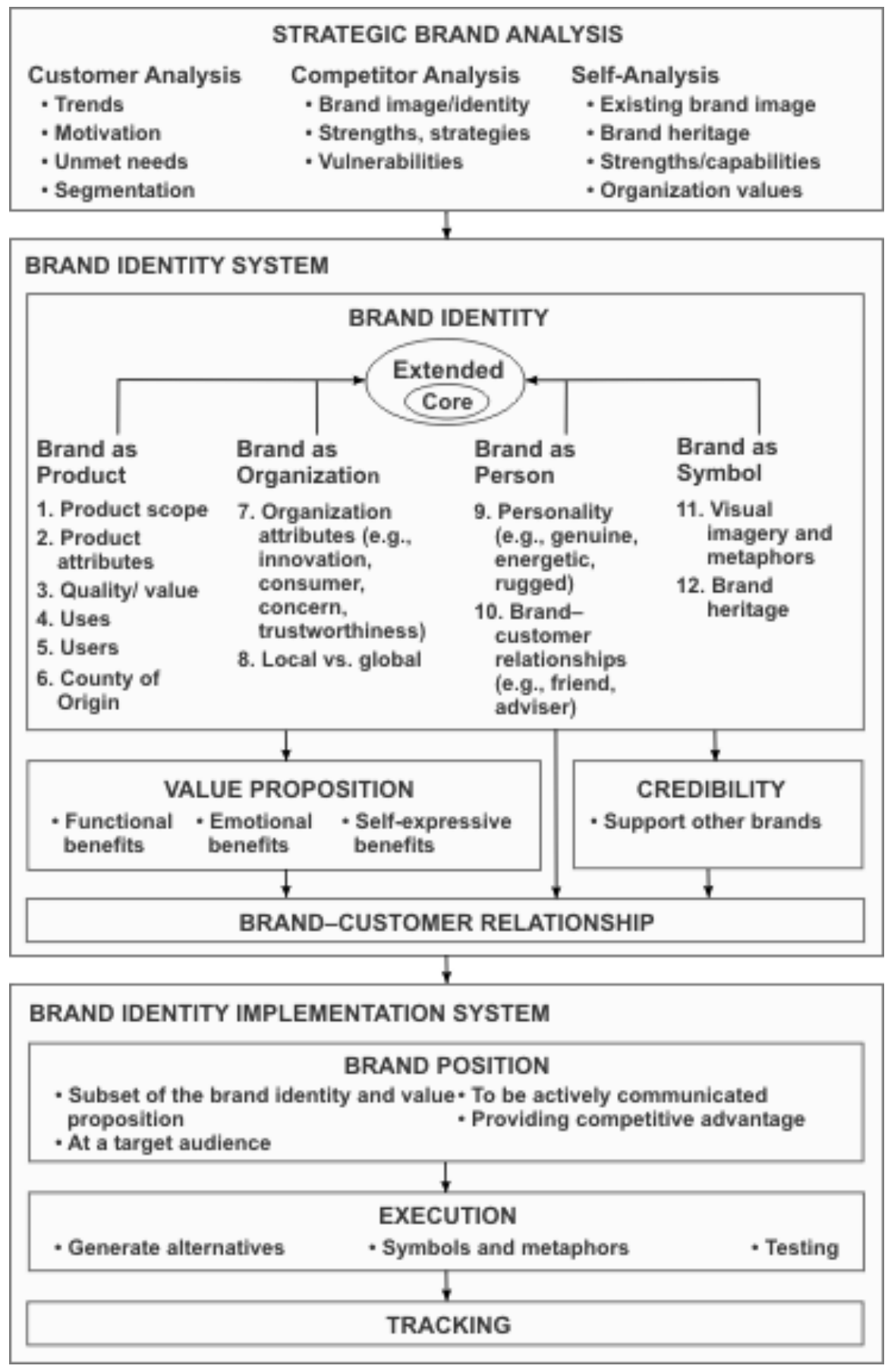

Figure 1. Aaker's (1996) brand identity planning model.

Aaker's (1996) model was unique in it was one of the first brand identity models to propose a brand did not need to employ all, or even several, of the subcategories of identity formation to be successful; a brand may only need to utilize a single category to articulate a solid identity. Prior to Aaker's model, it was assumed that brands often needed to employ a variety of 
measures to attract consumers (Kapferer, 1994, 2008). For instance, they could not focus on how their brand functioned as an organization alone, but rather how that organization helped to define the identity further. Aaker (1996) contested this by suggesting all brands have a core identity, one which is central to the meaning of the brand and is likely to remain constant as the brand grows, however, they also have an extended brand identity. These features focused on the brand as having depth and texture, not necessarily meeting a certain set of standard criteria (Aaker, 1996).

Following this notion of a core and extended identity, a brand may encompass one or more of the following perspectives: brand-as-product, brand-as-organization, brand-as-person, or brand-as-symbol (Aaker, 1996). The brand-as-product perspective suggested a brand may build its identity around the value added by products or services offered by the company (Aaker, 1996). This perspective proposed defining the tangible and intangible values of a product or service which cause consumers to remember the brand based on their needs (Aaker, 1996; de Chernatony \& Riley, 1997; Myers, 2003). For instance, a skin care company may suggest that their product reduced fine lines and wrinkles in a certain percentage of the population, proposing that the product would do the same for other consumers. If a consumer wishes to have the same results, he or she may remember these claims and seek out the brand based on their product offerings.

The brand-as-organization perspective focused on attributes of the organization, such as innovation, quality, and ethical considerations being defined by the people, culture, values, and programs of the brand (Aaker, 1996). For example, beauty brands that focus on being cruelty free and vegan may be more likely to attract consumers based on the organizational principles. Here the products could be secondary, as some consumers may trade product offerings for 
alignment with more intangible organizational beliefs. The brand-as-symbol approach proposed having a strong symbol to define the brand in order to gain recognition and recall with consumers (Aaker, 1996). Symbols are meaningful and can help establish other aspects of a brand's identity, such as Covergirl's tag line "Easy, Breezy, Beautiful” suggesting that their products symbolize these ideals. While the brand-as-product, brand-as-organization, and brandas-symbol are all perspectives that could be utilized to build a strong brand identity, this research sought to explore the brand-as-person perspective as it communicates aspects of social capital formation and identity matching through linking the human digital influencer to the intangible anthropomorphized assets of a brand.

Brand personality. The brand-as-person perspective suggested that like a person, a brand can have a strong personality (Aaker, 1996). Personality can work with other aspects of the brand identity, such as contributing to a functional product benefit (Aaker, 1996). For instance, the young and lively brand image of Nike is reflected in the brands brightly colored sneakers and apparel products (Aaker, 1996). The association between perceived personality of the brand and the products or services offered helps to build the brand-as-person perspective.

As Aaker (1997) proposed, brands hold certain personality traits related to those expressed by humans in face-to-face interactions. Research suggested relationships between the brand and consumer, as well as constructs of self-image, often mirrored these personality traits (Aaker, Fournier, \& Brasel, 2004; Fennis \& Pruyn, 2007). A sincere brand works to develop long-term relationships with consumers that will resonate with their self-images of being honest, cheerful, and down-to-earth (Aaker, 1997; Aaker et al., 2004; Fennis \& Pruyn, 2007). In contrast, a brand with an exciting personality will see more short-lived, but intense consumer 
relationships over time, as they correlate to self-images of being daring, spirited, and imaginative (Aaker, 1997; Aaker et al., 2004; Fennis \& Pruyn, 2007).

A brand with a strong personality also can help to foster congruency with the self-concept the consumer may use to express his or her own personality (Aaker, 1996; Dolich, 1969; Jamal \& Goode, 2001; Kressmann, Sirgy, Herrmann, Huber, Huber, \& Lee, 2006). Rosenberg (1979) defined self-concept as being the "totality of the individual's thoughts and feelings having reference to himself as an object" (p. 7). A self-concept is the subjective thoughts and views an individual has toward his or her self (Hong \& Zinkhan, 1995). While products and services carry symbolic values, each individual perceives them as holding different, unique meanings, which he or she may use to construct a subjective image of the self (Adam \& Galinsky, 2012; Dolich, 1969). Aaker (1996) suggested it is the meaning attached to these goods through the brand personality which drives consumers to purchase them as a way to express their identity. Brands work to build a personality reflecting an image of the typical consumer, which consumers use to match the brand to their own self-concept (Kressmann et al., 2006).

As brands develop a personality to meet some, or all, of these humanistic traits, they become more personable and like-minded with the consumer, promoting feelings of selfcongruency and fostering relationship growth with consumers (Aaker, 1996; Aaker, 1997; Kressman et al., 2006; Sirgy, 1982). Just as humans typically prefer interacting with individuals similar in personality to themselves, consumers prefer interacting with brands that share their personality traits. Developing a relationship with the consumer is becoming increasingly important in the fast-paced modern branding landscape and beauty worlds, as brands look to integrate new forms of brand extension with popular relationship-building tools. 
Brand migration to social network sites. With the advent of Web 2.0 technologies, namely social media sites, brands noticed a shift in how consumers gathered information (Alba, Lynch, Weitz, Janiszewski, Lutz, Sawyer, \& Wood, 1997). Consumers moved online to research brands and to purchase products as they gained a level of control over their consumption of goods and services (Alba et al., 1997; Cova \& White, 2010; Rohm, Kaltcheva, \& Milne, 2013). Consumers no longer had to rely on information to be distributed to them by the brand; they were able to connect among themselves and share experiences, separate of any proposed brand strategies (Rashid \& Ghose, 2015). Social network sites made it so brands had great difficulty controlling the reach of their messages, as consumers now were able to build content they deemed appropriate. By using these sites, consumers could interact with the content and share it across an ever-widening friend network. Brands who failed to understand the nature of social network site communication saw their content reach, frequency, and timing being controlled by consumers (Mangold \& Faulds, 2009).

The first wave of brands to enter these sites viewed them as another platform where traditional marketing and sales approaches would prevail (Fournier \& Avery, 2011). However, brands learned these sites held new power for the consumer. Companies who responded to negative feedback with comments such as "Thank you for your response. Please fill out a customer service contact form here, or call..." were touted as being robotic, not utilizing the communicative aspects of social network sites properly (Alton, 2015). Brands also experienced backlash when discourse was misconstrued through the multiple cultural communities found on social network sites. Kenneth Cole sparked outrage on Twitter in 2011 after posting the tweet, "Millions are in uproar in \#Cairo. Rumor is they heard our new spring collection is now available online..." (Garcia, 2011). The tweet referenced the crisis in Cairo after the start of the 
Egyptian Revolution in 2011. The comment was re-tweeted thousands of times with consumers suggesting the brand was insensitive and trying to use their social media reach to sell a product around a social issue. Although Cole issued a formal apology for the comment, consumers accused the Kenneth Cole brand of being insensitive and only focused on sales (Garcia, 2011). The post crossed the line into being inauthentic, and frankly, quite ill-advised. Brands quickly learned they had to change their marketing techniques to account for these new consumer communication affordances.

Eisenberg (2009) suggested the negative experiences by brands on social media are likely due to the fact they fail to employ the approach of "you [the consumer] talk, they [the brand] listen" (p. 139). In this version of the web, consumers have found a new sense of control over the content they consume and produce. If a consumer believes a brand is not being true to the nature of the networked society, placing their company success over the potential to build a relationship with consumers, they are quick to reject the brand (Kaplan \& Haenlein, 2010). Social network sites are platforms of communication, arenas in which users are able to connect with others and articulate their ideas. In terms of branding, social network sites have created an interesting paradox for retailers, one where everyone and no one can be the audience at the same time (Fournier \& Avery, 2011).

While some brands struggled to reach audiences properly through social network sites, others realized important aspects of the brand/consumer divide provided by these platforms. Warhammer, launched in 1983, is a strategic game played with miniature characters on a battlefield. Since that time, the game has amassed millions of players and is considered one of the largest and most successful tabletop games in the world (Cova \& White, 2010). Buying Warhammer products placed the consumer into a community extending far beyond the functional 
use of the product itself. Online chat forums for the game evolved with Web 2.0, where players could meet virtually to discuss how the game was being played in a global context. Games Workshop, the company who owns Warhammer, began engaging with consumers on these forums, asking for opinions on the current game play and searching for new ideas players may have (Cova \& White, 2010). This open platform allowed the brand to share with consumers, cocreating value and developing a virtual community of users. The information shared on these sites seemed safe and comfortable to the consumer, as they were interacting with the brand on a more intimate level (Rohm, Kaltcheva, \& Milne, 2013). The company was not trying to use the site to sell additional products to consumers but rather to build a relationship with them. It is personable connectivity on social network sites which often gain the most positive consumer responses, as it suggests companies want to have a true relationship with them, the hallmark of social network platforms.

This personal connectivity of social network sites, combined with the exchange of social capital, is guided by the reality of the communities and participants themselves. Brands see success on social network sites when they recognize the influence of the community, positioning themselves as being a part of it, not trying to redefine it. In a similar sense, individuals have joined these communities as they see the benefit in being part of them. There is an exchange of resources through the maintenance of social capital, however, there is also an element of reality in these communities. Individuals are not actively trying to dupe others into thinking they are something else on social network sites, as being unreal is not desirable in these communities (Baym, 2010). Instead, these communities thrive on members being true to their self and standing true to their promises, creating an authentic personality reflective of their reality in the digital environment. 


\section{Defining Authenticity}

In this study, authenticity is defined as the operation of one's true self in everyday life, as presented through genuine portrayals of identity constructs. This definition is built from the wide breadth of research addressing how authenticity is projected. Kreber, Klampfleitner, McCune, Bayne, and Knottenbelt (2007) found perceived authenticity in teaching is associated with presenting a genuine, credible, reflective self. Sloan (2007) suggested a similar definition of authenticity, in being perceived as authentic is a commitment to expressing the self freely. Henderson and Bowley (2010) proposed authenticity is associated with concepts of the self in a social context. A common thread ran between each of these definitions - that authenticity is determined personally and experienced - based on subjective views of the reality an individual has constructed (Gilmore \& Pine, 2007).

Similar to developing social capital, authenticity is constructed through subjective constructs. What one individual deems as authentic behavior may be determined inauthentic by another. The determination of authenticity is ultimately presented as a measure of a whole, where if a majority deems a person, brand, or service authentic, then the community will follow suit. Gilmore and Pine (2007) proposed this is the effect of operating in a world of real and fake identity projections.

Gilmore and Pine (2007) suggested authenticity is based on "being true to your own self" and "being who you say you are to others" (p. 111). Gilmore and Pine (2007) suggested assessing the value of being real and/or fake, in relation to projections of perceived authentic offerings. Being real is defined by being true to your own self and being who you say you are to others, while being fake is defined by the inverse of these statements (see Figure 2, p. 41). It is integral to note Gilmore and Pine (2007) did not situate being fake as inauthentic. Instead, being 
fake is defined deliberately and is often a function of escapism. Situating elements of the identity as fake often prescribe to notions of self-motivation, as being true to yourself or doing what you say you will do may not best serve your individual needs. Thus, projecting a fake authenticity is not in an effort to damage others, but rather to generate meaning in self-motivated means.

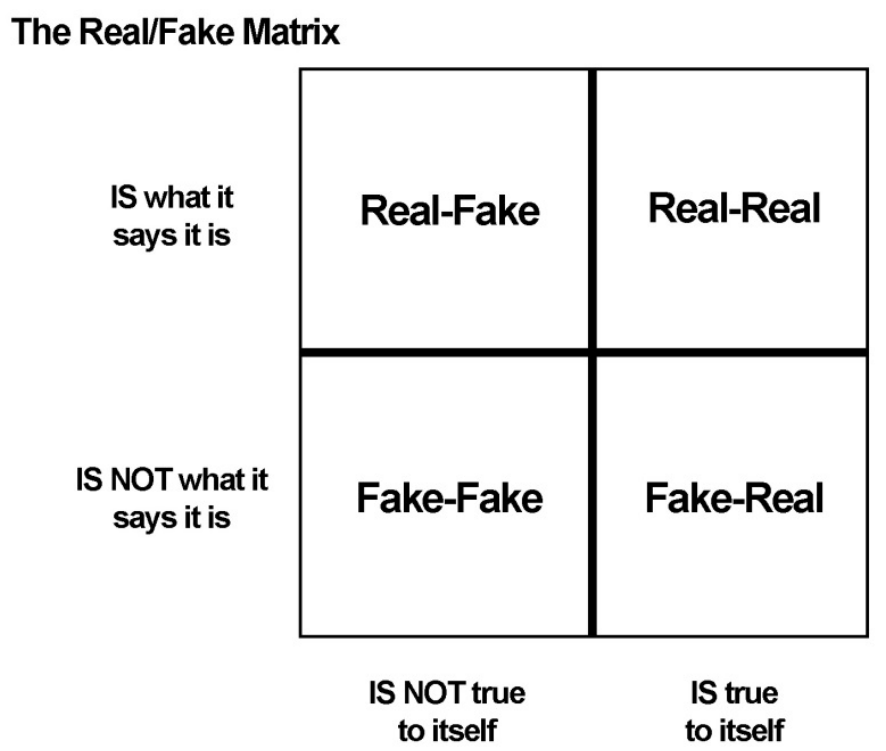

Figure 2. Real-fake matrix as proposed by Gilmore and Pine (2007).

As long as the individual acknowledges real and fake qualities, it is still considered an authentic experience. It is when the individual does not acknowledge his or her reality properly that questions of authenticity arise. Gilmore and Pine (2007) suggested having a strong sense of the type of authenticity you wish to project, regardless of whether it appeared real or fake, is deemed the most authentic by others. Thus, maintaining one type of real and/or fake projection across a variety of settings will produce the most authentic results, as the individual expresses the self freely.

The notion of authenticity typically is associated with concepts of the self within a social context (Henderson \& Bowley, 2010). Authenticity is assessed locally and temporarily, based on the context of individual and societal views of reality at the time (Henderson \& Bowley, 2010; 
Marwick \& boyd, 2010). Prior to social network sites, the idea of an individual being authentic was based on contextual clues in face-to-face communication (Henderson \& Bowley, 2010; Marwick \& boyd, 2010). However, mediated communication reduces the possibility for nonverbal and context clues to help define actions as being authentic.

Social network sites and authenticity. In a mediated context, the projection of real and fake authenticity constructs is often assessed through the reciprocity of actions. Livingstone (2008) found individuals using social network sites rated others as being more or less authentic based on the reciprocity of their actions. One participant noted he could tell whether an individual was real or not based on the pictures they posted and if they were willing to comment on others posts. If they were simply looking to gain a high number of virtual friends and did not want to interact with others, he deemed them as being inauthentic (Livingstone, 2008).

This relates back to Gilmore and Pine's (2007) assessment of real and fake authenticity projections. Members of a digital community are not able to assess authenticity from any facets other than those disclosed by the user; authenticity projections must be taken at face value. If an individual engages in a digital community and projects herself as being real-real (see Figure 2, p. 41), then she should be engaging in selfless acts true to her position in the community. For instance, she should be liking images from other members or engaging in conversation about their lives. If she did not reciprocate these actions, but asks for them from other members, she is instead engaging in fake authenticity projections.

If her motivation for engaging in the community is to further her own self-interest, while still remaining true to who she is, her authenticity would be defined as fake-real. To avoid being labeled as inauthentic, she should define this intention from the beginning. In a digital community, letting others know your intentions is acceptable, as disclosure is left to the 
individual and engaging in communities can become a very complex process (Baym, 2010). As Gilmore and Pine (2007) suggested, individuals engaging in these digital practices should be upfront about their authenticity motivations. If they wish to gain some self-centric asset from their engagement in a digital community, these motives will be received better if they are present from the beginning of the interaction (Gilmore \& Pine, 2007). Thus, being authentic in a mediated context does not require complete pureness but rather is built on the notion individuals will be transparent in their interactions and define their intentions through their disclosure to others.

In assessing digital environments and authenticity, the focus on the individual is often of primary interest, however, social network sites have a variety of entities interacting on these sites. As brands migrated to digital social networks in an effort to engage with consumers, they positioned themselves as being part of these communities, which in turn required they define their authentic offerings to others.

As individuals recognized the ability to generate and discern between authentic and inauthentic personal communications on social network sites, they also began to challenge brands to become authentic. These concepts of authenticity also may be applied to brands, as it has crafted an identity which expresses its self (de Chernatony, 1999; Kapferer, 1994).

The first iterations of building an authentic brand online were focused on the idea of providing consumers less-contrived experiences, focused on elements of self-congruency as brand building features (Gilmore \& Pine, 2007). Websites such as NikeID.com and zazzle.com allowed consumers the opportunity to create a unique product and displayed elements they thought directly impacted their self-image. While the items were still being designed within the confines of product offerings on the sites, these customization efforts shifted the focus from sales 
motives to pursuits of an ideal self-image (Gilmore \& Pine, 2007). These did not work, however, to address the issues of being "real-real" in a mediated context. Brands wanted to be who they said they were, while still being true to themselves and selling products. Traditional marketing approaches often overlooked the reality of a product or service, instead focusing only on the positive attributes, causing a disruption in the real-fake matrix. With the advent of social network sites, brands realized they could further explore defining authenticity through the tool which built the social networks - the community members themselves.

\section{Consumer-Generated Advertising and Branding}

The advances in social network technologies put a level of control back into the hands of the consumer (Mangold \& Faulds, 2009). The consumer could research a purchase with ease, communicate its identity with others, and form opinions of a brand with a relatively low amount of risk (Rashid \& Ghose, 2015). While brands initially struggled with the incorporation of usergenerated content (UGC) into their marketing plans, they noticed a new affordance emerge, consumer-generated advertising (CGA) (Ertimur \& Gilly, 2011).

CGA, much like other forms of consumer-driven content, has the ability to engage with audiences and create personal connections. Possibly the most prolific example of CGA is of the YouTube review video. Tolson (2010) proposed YouTube is no longer solely used for entertainment, but as a form of information gathering and directed communication. Consumers are able to utilize the YouTube platform to generate feedback about a brand, product, or service, as well as generate dialogue with other users by commenting, which is then broadcast to a global platform. With blogs and social network sites accounting for nearly $25 \%$ of the time consumers spend online, CGA has the ability to reach a broader market than traditional forms of advertising (Bennett, 2013). 
At the peak of CGA is the digital influencer. A digital influencer is an individual who has gained a heightened digital presence through platforms such as blogs and social network sites, making his or her words reach across more diverse and larger communities (Senft, 2008). Differing from larger scale celebrities, these individuals only are famous to a niche group, one that allows the influencer to feel similar and connected to fellow community members (Booth \& Matic, 2011; Marwick, 2013). An influencer is able to affect perceived brand ownership through interacting with users and deciding what type of information is projected to viewers (Booth \& Matic, 2011). As brands realized social network sites made it difficult for them to control the information being discussed and presented among consumers, they started looking to influencers as powerful generators of CGA, with the ability to build relationships and enable conversations in a digital marketplace, all the while remaining a regular individual (Booth \& Matic, 2011).

Prior to CGA content, brands often looked to celebrities for endorsements. The cultural values, norms, and characteristics embedded within an advertisement are crafted in a way where viewers are able to find similarities between the characters in an ad and their personal selfconcept (Hong \& Zinkhan, 1987; Krampf \& Ueltschy, 1997). However, Tolson (2010) found in the modern advertising landscape, consumers note they identify more with individuals on YouTube or other social media influencers, as they present an air of transparency. The influencers utilized in CGA are not celebrities, but rather everyday individuals, who are able to communicate directly with the viewer and provide beneficial information. There is the possibility of generating bridging and bonding social capital with these content generators, as they are trusted and relatable. Their influence reaches far beyond the scope of traditional advertisers, as they build capital with viewers and promote elements of an authentic personality. These 
affordances have allowed for digital influencers to permeate a new market, generating followings in their respective communities and having considerable impact among viewers.

CGA, beauty branding, and influencers. In regard to digital influencers, the beauty industry is one content area which has seen a tremendous growth in brand and influencer success. With over 6,500,000 global YouTube channels dedicated to beauty and its top beauty influencers amassing more than 10,000,000 million viewers, the beauty marketplace has seen a revolution through influencer engagement (Mosendz \& Bhasin, 2016; YouTube, 2016a).

While the costs associated with engaging in beauty practices are focused on individual meanings ascribed to the experiences and products, this cycle of purchasing does not exist in a vacuum. Cosmetic companies have to rely on careful advertising and brand name recognition to attract and retain a customer base (Kumar, Massie, \& Dumonceaux, 2006). Established cosmetic brands must contend with changes in product offerings, a societal focus on health and safety of products, as well as the competition of new, innovative brands using specialized ingredients in their products (Kumar et al., 2006; Mansor, Efni binti Mat Ali, \& Yaacob, 2010). As more individuals focus on the application of make-up and beauty practices as being experiential in nature, helping to identify part of the self through utilizing the body as a project, the building of a relational cosmetic brand becomes increasingly important to obtaining a strong consumer base. While the products being sold by the cosmetic industry are grounded in consumption, the meanings and roles they promote are uniquely constructed by the individual and society within which they are consumed (Johnston \& Taylor, 2008). The modern cosmetic industry no longer seeks to simply sell a product but to "sell us ourselves' in an environment 'within which we and those goods are interchangeable"” (as cited in Merskin, 2007, p. 595). 
One way in which the cosmetic industry achieves this is through the incorporation of influencers into branding campaigns. Companies seek out influencers as a means to link consumers to the brand's content (Spencer, Harding, \& Sheahan, 2014). Social network site influencers have grown their personal brands, using their status on these sites to manage authenticity (Gillin, 2007). While these influencers may have amassed tens of thousands of followers, they are still considered relatively small niches in comparison to celebrities and other forms of mass media, such as television and magazines. However, in a mediated context, honing a small niche market can be extremely successful to personal brand growth, as consumers consider your communication to be authentic. For instance, social network site influencers typically are more likely to respond to their followers than larger celebrities or media formats, furthering the ideals that this is a reciprocal relationship.

As the credibility and trustworthiness of an influencer are established, brands begin to view them as being potential ambassadors to their products (Booth \& Matic, 2011). As the influencers build relationships with their viewers, they also may begin to extend information about the brands they consume. The influencers are able to create bridging social capital through the information being shared. As they continue to gain followers based off this initial content generation, the directed communication allows the influencer to begin forming bonding social capital through the trust and reciprocity of users. As Gillin (2007) proposes, having an influencer link a product or service to their personal brand is a sign of validation, a way of suggesting they respect the brand and encourage their followers to do so as well. There is an inherent level of trust garnered through the authenticity of the content generator. However, as influencers realize their power in the branding process, many are becoming more professional in their dealings with companies. There is now a commoditization of opinions and content in the beauty community of 
YouTube, in which influencers can leverage their impact among viewers to garner additional resources for themselves.

\section{Influencer Impact among Viewers}

Influencers are able to maintain credibility with fans through their established digital presence. However, they also may offer their services directly to brands, sharing with them data such as audience composition and promoting their products in content in return for payment or products (Abidin \& Ots, 2015; Griffith, 2011). As Rocamora (2012) proposed, new media can refashion old media, such as the perceived authenticity of an influencer holding precedence over the spirited past of a brand. Consumers are skeptical of traditional advertising techniques as the original source of the message comes from the brand directly, in an attempt to persuade consumers to purchase a product (Calfee \& Ringold, 1994). In CGA, the message comes from an individual, a new form of advertising in the digital context, where elements of social capital and authenticity help to construct a "realness" around the message being conveyed. Yet, this pushpull between remaining true to the user and monetizing the personal brand has proved a struggle for some content generators.

Bloggers have noted they want to be truthful to their audiences — as they gained many of their followers prior to partnering with brands-however, they also feel an obligation to the brand (Abidin \& Ots, 2015). In a study by Abidin \& Ots (2015) one blogger mentioned she navigates this divide through her dialogue to readers. She noted she was once sent a dress so thin and transparent you could see directly through it, however, she had agreed to be photographed in it as compensation for the product. She used descriptors such as "light" and "airy" to define the dress, suggesting it was a good layering piece, insisting that by telling her consumers these suggestions she was not lying to them nor was she discrediting the brand's product (Abidin \& 
Ots, 2015). This blogger understood that readers were sensible and could tell the dress was transparent, however, she gained financially by promoting the item. Her tactic, as employed by many influencers, was to acknowledge the shortcomings of the dress, while showing how it still could be a great investment piece. These influencers typically are not required to read a script from the brand regarding a product, as CGA dictates the influencer provide his or her own interpretation on branding practices. However, if the influencer is being paid for the content, there is a certain expectation by the brand. The product should be placed properly in the content, while still maintaining the authentic, honest image of the influencer to the user. As bloggers begin to monetize their influencer status, it seems they start to toy with the notion of being authentic, yet still making a profit.

One blogger in the study by Abidin \& Ots (2015) demonstrated this damage to her credibility and authenticity based on a sponsored post. The blogger worked with a brand to promote a bust-enhancing cream, however, some followers soon took to the blog to complain their results did not match hers. Although the blogger had clearly marked the post as being sponsored by the beauty company, and it appeared to be the fault of the cream to not work as advertised, followers still thought they had been lied to by one of their own. Several readers accused the blogger of simply trying to make money off their loyalty and being irresponsible to her readers (Abidin \& Ots, 2015).

Even though brands often attempt to reveal their sponsorships through creating hashtags or asking influencers to create content with their brand name in the tagline there are still questions regarding authenticity and full transparency on social network sites in regard to influencers (Burns, 2016). A new element of CGA was introduced when the Federal Trade Commission (FTC) began to require full transparency regarding sponsored video posts. The FTC 
requires influencers make their affiliations known to users, when being paid for endorsements on behalf of a sponsoring advertiser (Federal Trade Commission, 2015). The FTC considers an endorsement when an advertiser "pays [the influencer] or gives [the influencer] something of value to mention a product" (Federal Trade Commission, 2015, n.p.). If the influencer is being paid to create content specifically for a brand, he or she must make the relationship clear to the viewers to avoid deception.

The guidelines regarding free products and affiliations are not as clear. The FTC notes influencers should let their viewers know if they are receiving products from a company with the expectation of review (Federal Trade Commission, 2015). However, many beauty influencers are sent free-products from brands as public relations packages, with no expectation the influencer will create a video about the products (Strugatz, 2016). Instead, the brands use these products as a way to engage with the influencer, toward a potential sponsorship in the future, as influencers continue to gain notoriety and can be selective in whom they partner with (Strugatz, 2016). In this aspect, there is no requirement for the beauty influencer to announce a product was sent to her for free, as there is no expectation it be promoted in her content. These wavering requirements on being transparent in regard to branding and business relationships have furthered the power of the influencer. These individuals now can be selective in what information they project to the user and when they choose to do so, however, this can cause problems with authenticity and social capital among users, as it creates the appearance of influencers being a paid member of the community, not a true member.

\section{Summary of Study Based on Literature Review}

As influencers navigate the careful path between regular consumer and brand ambassador, it is unclear how they manage their authenticity and social capital, toward 
projecting an image beneficial for themselves, the viewers, and the brands with whom they partner. This study investigated how influencers in the YouTube beauty community project authenticity and maintain social capital in regard to monetization of content, in an effort to address this gap in knowledge. This study utilized a digital ethnography framework to conduct a content analysis toward gaining an understanding of how these constructs were projected and constructed by the influencers. The research also assessed how social capital is portrayed, both in bridging and bonding forms, in conjunction with monetized and non-monetized content. In addition, this study addressed how beauty influencers function as elements of beauty brands, furthering their own personal financial gains through sponsored content, as well as how they may impact beauty brand identity. This exploration was an effort to better understand beauty influencers' role in branding mechanisms through their consumer-generated advertising standing. Finally, an assessment of how viewers respond to these constructs was included, with an additional look at the communicative practices within the community as a whole, in an effort to understand how monetization affected beauty influencers within the digital community. Through this study, a better understanding of how these constructs may be impacted by monetization was addressed. A discussion of the methods utilized to complete this research are discussed in the following chapter. 


\section{CHAPTER III: METHODOLOGY}

This study explored how authenticity is constructed and classified, as well as how social capital is maintained by beauty influencers on YouTube. This research also sought to understand how these concepts are modified by the influencer and received by the viewers in regard to sponsored brand activities. Social network sites have provided a platform for participatory culture, where viewers are able to express themselves, creating and sharing content (Jenkins et al., 2006; Kaplan \& Haenlein, 2010). These sites changed the landscape of the web from passive browsing to active participation (Cormode \& Krishnamurty, 2008).

YouTube furthered this participation, allowing for the inclusion of audio and video to textual content generation, incorporating elements of personal communication and building an influential "self" online (Booth \& Matic, 2011; Marwick, 2013). As beauty influencers on YouTube constructed a self through a variety of constructs, they built rapport with the viewer, from which authenticity is defined by subjectively building and maintaining social capital with viewers (Henderson \& Bowley, 2010; Gilmore \& Pine, 2007). This authenticity was further tested and refined as YouTube beauty influencers engaged in brand building activities through sponsored posts by beauty brands, as well as promoting affiliate products. As Gilmore and Pine (2007) proposed, authenticity can be constructed around commodities, goods, and services, however, it is experiences and transformations which allow for the subjective development of the self. It is elements of trust and influence put forth by digital influencers that allowed them to maintain bridging and bonding social capital, which ultimately could be utilized to project authenticity within a community.

As beauty influencers on YouTube continued to construct an authentic self and maintain social capital, as well as engage in brand-identity driven content generation, it was important to 
understand how these were constructed and maintained. This research utilized a qualitative study design to explore the main research question (RQ) and four sub-questions (SQ):

RQ1: How do YouTube beauty influencers maintain social capital and project authenticity while engaging in monetization activities?

SQ1: What practices are employed by YouTube beauty influencers to construct and maintain perceived social capital?

SQ2: What practices are employed by YouTube beauty influencers to construct and maintain perceived authenticity?

SQ3: How do YouTube beauty community viewers respond to monetization activities through the comments section offered by YouTube?

SQ4: How do YouTube beauty community viewers respond to and interact with beauty influencers' social capital and authenticity projections through the comments section offered by YouTube?

In addressing these research questions, it is necessary at this point to reiterate the operational definitions of beauty influencers, beauty community viewers, social capital, and authenticity as to their context in this research. Beauty influencers were defined as individuals who had generated a notable digital presence through their engagement in beauty-related content generation and were able to utilize their impact to position themselves as relevant in the beauty community. As this study directly addressed YouTube beauty influencers, this definition reflected their status in the YouTube beauty community. Beauty community viewers were individuals who viewed beauty influencer video content and engaged in active or passive consumption. The constraints of this study did not allow for a full exploration of community member practices, such as delineating the frequency of involvement in the community by the individual. Additionally, as YouTube does not require viewers to create an account or display their membership status to the site, any individual could be a viewer of this content. This study utilized Bourdieu's (1985) definition of social capital as the acquisition of shared resources 
through the interactions of a members in a network as the basis for which social capital was assessed. Elements of Sander and Lee's (2014a) digital social capital model were utilized to assess social capital in a mediated context, as influencers engage in specific practices on social network sites to build and maintain social capital. In this study, authenticity is defined as the operation of one's true self in everyday life, as presented through genuine portrayals of identity constructs (Goldman \& Kernis, 2002). This is further defined from Gilmore and Pine's (2007) authenticity matrix, which assesses whether individuals are true to themselves and if they do what they say they will. These four definitions serve as the basis for operationalizing the constructs explored in this research.

In addition to determining operational definitions, it is also essential to discuss where this study finds itself practically. While the theoretical underpinnings of this research will be discussed here-in, this study explores a practical element of modern society: the social network site and its permeation into culture, both real and imagined. As individuals migrated online to build digital personas and develop relationships beyond geographic boundaries, multiple researchers addressed the personal impact of communication and relationship building in a digital context (Baym, 2010; Booth \& Matic, 2011; Tolson, 2010). Research has also been conducted on how social network sites have impacted corporations in terms of with consumers (Kaplan \& Haenlein, 2010; Mangold \& Faulds, 2009). However, social network influencers have bridged both sides of social media, the personal and the business, of which the impact and effects have been less researched in regard to the building and maintenance of digital cultural practices and forms, toward creating a digital community. This research sought to address these topics, toward examining the interactions that functioning as both an individual and a personal brand 
can have on influencers and their communities of interest, in an effort to better understand the sustainability of such practices.

Specifically, there is one group of digital influencers who have seen great success in marketing their personal brands: the beauty influencers. These men and women have succeeded in creating expansive digital communities, with over 78 "how-to and lifestyle" YouTube channels amassing more than 2,000,000 subscribers each (VidStatsX, 2016). Beyond their subscriber counts, beauty influencers have generated great personal revenue from their channels. In 2015 it was estimated that the highest paid YouTube beauty influencer generated an average of $\$ 41,000$ monthly just from her channel's content (Richtman, 2015). Beauty influencers have launched their own cosmetic brands, partnered with companies at international beauty destinations to create products, and have even been featured on televisions shows and magazine covers based on their digital notoriety (Mosendz \& Bhasin, 2016; Richtman, 2015). While their success has been noteworthy, it is yet to be addressed how these monetization activities affect the original community that built their notoriety: the digital beauty community.

This research drew upon a digital ethnography framework to guide an inductive content analysis of video content and text-based comments. These methods were best suited to answer the research questions, as they aligned most closely with qualitative methodology and a constructivist epistemological stance.

\section{Paradigmatic Assumptions}

Constructivist research builds on the principles that multiple realities exist, reality is socially constructed, and the linkages of the investigator to the object of study, thereby emphasizing process and meaning (Sale, Lohfeld, \& Brazil, 2002; Tashakkori \& Teddlie, 1998). Research of this type operates under the assumption there are multiple, constructed realities 
which are bound by the context in which they are experienced (Tashakkori \& Teddlie, 1998).

Constructivist research follows the epistemological stance the researcher and participant are inherently linked (Sale, Lohfeld, \& Brazil, 2002; Tashakkori \& Teddlie, 1998). While the influencers and viewers provide the data, I, as the researcher, helped build knowledge around data. The interaction itself created an element of connection allowing for knowledge to be constructed through both construction and interpretation. In the context of this study there was no personal interaction between the participants and researcher, but rather a perceived connection through my inclusion in the digital beauty community. Additionally, I acknowledge inquiry as value bound (Tashakkori \& Teddlie, 1998). Individual values of each influencer will have an impact and should be taken into consideration when completing constructivist research. Each of these values should be explored within the context of the reality the influencer has created, suggesting knowledge generation is bound by individual values, which then may be compared across multiple realities.

Based on the axioms of ontology, epistemology, and axiology, I operated under a constructivist paradigm to complete this research. Based on my constructivist paradigmatic beliefs, digital ethnography allowed for the exploration of culture by focusing on how the digital environment was utilized in shaping behaviors, interactions, and cultural processes (Ardévol \& Gómez-Cruz, 2014; Hine, 2007; Masten \& Plowman, 2003; Murthy, 2008). Through this research, I explored how YouTube beauty influencers constructed realities through authenticity and social capital, as well as a larger interpretation of how that process developed within a specific time and context. I utilized qualitative research based on my constructivist paradigmatic assumptions; subjectivity will be explored in this chapter through detailed exploration of the selected theoretical framework and methodology. 
Selection of qualitative methodology. Qualitative inquiry employs a variety of philosophical assumptions, inquiry strategies, data analysis techniques, and interpretations. A qualitative approach to research does not define a specific set of measures which must be adhered to or a stringent interpretation technique. Denzin and Lincoln (2005) proposed that qualitative researchers should, “...seek answers to questions that stress how social experience is created and given meaning" (p. 10). This suggests while there is no specific definition given to this type of research, as it is bound by context. At its essence qualitative research, "...involves an interpretive, naturalistic approach to the world," with researchers, “...attempting to make sense of, or interpret, phenomena in terms of the meanings people bring with them" (Denzin \& Lincoln, 2005, p. 3). In qualitative research emphasis is placed on determining the concepts, definitions, characteristics, metaphors, symbols, and descriptions of meaning within the context of time and space (Berg, 2008). Qualitative research often employs inductive research strategies to immerse the researcher into the details and specifics of the data to explore questions regarding a phenomenon, not strictly testing theoretically-driven hypothesis (Creswell, 2009).

As time and space are contextually bound in qualitative research, Creswell (2009) proposed researchers locate their data in everyday life, rather than in strict laboratory settings. Creswell (2009) also suggested qualitative researchers play a central role in the research process, acting as the primary research instrument for data collection. Therefore, my paradigmatic assumptions served to provide a shared level of meaning between the subject and phenomena, placing emphasis on context (Creswell, 2009; Denizen \& Lincoln, 2005). This approach sought to understand the whole picture of the social context under investigation, in which the sum is greater than the parts. 
This research also utilizes the researcher as an instrument, with myself functioning as a tool in the research process (Hammersley \& Atkinson, 2007). The researcher-as-instrument approach takes into account the social spaces in which qualitative research is often performed, including the digital space utilized in this study (Pezalla, Pettigrew, \& Miller-Day, 2012). Turato (1995) proposed that this allows the researcher to "use their sensory organs to grasp the study objects, mirroring them in their consciousness, where they then are converted into phenomenological representations to be interpreted" (p. 510). To assert quality and rigor in this instrument, researchers must be open about their practices, addressing any potential biases upfront and remaining cognizant of the reflexive nature of this style of inquiry. Researchers have suggested that this practice also helps others to hone their qualitative inquiry practices, as the self-reflexivity demanded of the researcher-as-instrument employs a variety of tasks and skills transferable across a wide range of qualitative inquiry methods (Pezalla et al., 2012). The researcher-as-instrument approach allows me to assert my position as a member of the digital space and community within which this research was conducted, permitting me to use my own knowledge and cultural understanding to convert representations into interpretations.

To explore authenticity and cultural capital by beauty influencers on YouTube, I had to place the questions within the specific context of time and space upon which they were evolving to obtain a holistic view of the phenomena. This combination of paradigmatic assumptions, methodological underpinnings, and research questions were best explored through the use of a digital ethnographic content analysis. Digital ethnography served to study the cultural connections of a networked society, where behaviors and interactions of the viewers worked to construct a digital context around shared practices (Caliandro, 2016). 
The internet as an object of study, functions as both a cultural form and cultural practice (Hine, 2000). As a cultural form, the web provides a unique system of norms and values, where "sense[s] of self, belonging, and group socialization can be shaped significantly by digital media" (Ardévol \& Gómez-Cruz, 2014, n.p.). In regard to the internet as a cultural practice, these instances are not specific to the web, but rather defined uniquely by digital dimensions (Ardévol \& Gómez-Cruz, 2014; Hine, 2000). The internet does not necessarily “create” culture but instead provides a new context to offline cultural practices.

When assessing beauty influencers on YouTube, the internet provided both cultural forms and practices. The influencers built a persona on the platform, providing a sense of self and inviting group socialization through communities of interest, fostering the platform as a source of cultural form. At the same time, the platform provided a means of assessing cultural platforms. The information being presented by these influencers was not bound by the digital media, whereas magazines and television programs have promoted beauty products and routines for decades (Brown, 2008). Instead, YouTube offered a context through which cultural practices could be defined by new dimensions, such as participatory culture, authenticity, and brand management by the viewer. As culture can be both created and re-defined in this environment, a digital ethnography provided a means by which to assess digital culture using classic ethnography methods, beyond geographic and temporal boundaries (Masten \& Plowman, 2003).

\section{Digital Ethnography as Theoretical Framework}

Research in mediated communication has evolved and advanced from a focus on studying things to studying actions, in contextualized and cultural practices (Varis, 2016). Digital ethnography as a theoretical framework builds upon pre-digital ethnographic practices. As Geertz (1973) proposed through his thick descriptions, ethnography takes interest in the lived 
reality of humans within a culture, aiming to produce detailed accounts. As Ardévol and GómezCruz (2014) suggested, "Ethnographers must attend to people's sayings and doings, including their material condition of existence and their worldviews: how people build meaning in relation to their experiences and actions" (n.p.). Ethnography is a learning process, where discovery is an extension of what human beings do naturally: "learn the meanings, norms, and patterns of a way of life" (Varis, 2016, p. 58).

As the world become increasingly more digital, it is imperative researchers understand the affordances of these sites and native cultures which emerge through the platforms. Digital ethnography provides a framework upon which researchers can study the cultural conditions, both in form and practice, of the computer-mediated networked society (Caliandro, 2016). The tenants of digital ethnography do not vary from those of traditional ethnography, as it serves to describe how the digital environment and shared connectivity can shape behaviors, interactions, and cultural processes (Caliandro, 2016). As Murthy (2008) proposed "'everyday life' for much of the world is becoming increasingly technologically mediated" and "new media and digital forms of 'old media' are additional, valuable methods" (p. 849).

Digital ethnography puts participant observation back in the hands of the participants, conveying the real-time richness of participants lives, experiences, and environments (Masten \& Plowman, 2003; Varis, 2016). This control is provided through the main field site of digital ethnography: the digital public (Caliandro, 2016). Digital publics are defined as social spaces constructed through the networked technologies and platforms, but also by the imagined collective cultures emerging through viewers' interactions and practices (boyd, 2011). Members of a digital public do not remain connected through direct interaction but rather by an imaginary culture created and elaborated upon by members and spread to those within the public 
(Arvidsson, 2013). This creates an environment where a "social space generated by the reflexive circulation of discourse" functions as an element of shared digital culture (Caliandro, 2016, p. $673)$.

While some scholars have suggested the online environment is a shared community, the concept of a digital public allows for the ethnographer to explore extreme interactions on social network sites, as these instances are not always as persistent, dense, or structured as those in a community (Postill, 2008). For instance, the interactions between players on a private video game forum are classified as community discourse, because the players have shared practices and communicate frequently with each other (Cova \& White, 2010). There is an expected element of density, persistence, and shared group think among these members as the private nature of the forum denotes members must gain acceptance into the group before discourse may proceed (Cova \& White, 2010). In comparison, social media sites have lower barriers to entry and communication, creating much looser relationships with varied levels of density and structure (Caliandro, 2016; Ellison et al., 2007). The information is still created and shared among viewers, however, the context and structure may vary greatly among members of a digital public. Digital publics often focus on participation as a form of self-presentation, where membership in the public is not based on roles played within a defined community setting (Marwick \& boyd, 2011). The notion of a digital public allows the ethnographer to explore these less defined interactions from a wider cultural lens rather than defining them to a specific community of viewers.

Digitalization has provided unique affordances for how the researcher is able to gain access to these publics and explore their cultural interactions. Rogers (2013) proposed the importance of digital ethnography is its grounding in digital methods. Rogers (2013) made the 
careful distinction between digital and virtual methods, noting virtual methods typically are developed offline and then applied to an online environment. Digital methods instead take into account "the nature and affordances of the digital environment" (Caliandro, 2016, p. 672). This requires the researcher to follow the natives, understanding that the nature of the social media platform and the actors' activities on these sites are essential to having a holistic view of the phenomena (Caliandro, 2016). While the design of the platform does not specifically determine how a viewer will engage with it for communicative practices, the physical design of the site can influence interactions (Varis, 2016).

As van Dijck (2013) proposed, sites which are designed to promote sharing and following among public members can have profound social values in cultural practices. Individuals are more inclined to "like" images on Facebook that have generated a high amount of interest already; these users are four times as likely to comment on another individual's post than they are to create their own unique content (Hampton, Goulet, Marlow, \& Rainie, 2012). This is due to the notion these sites provide physical features to promote connection between users, such as highlighting the "like" button or showing a preview of "comments" on another's post (van Dijck, 2013). Digital methods explore these affordances, in addition to elements of communication, thereby positioning the platform as being essential to understanding the culture of the web. Digital ethnography methods allow for the conceptualization of these sites in new contexts, allowing the study of digital environments and their functions as a way to observe social formations, cultures, and shared identities among members of the digital public.

Ethnographic content analysis. As Varis (2016) proposed, ethnography is a learning process, which then may be explored through a variety of methods. Altheide (1987) suggested Ethnographic content analysis is used to document and understand the communication of meaning, as well as verify theoretical relationships. Its 
distinctive characteristic is the reflexive and highly interactive nature of the investigator, concepts, data collection and analysis. Unlike QCA [quantitative content analysis] in which the protocol is the instrument, in ECA [ethnographic content analysis] the investigator is continually central, although protocols may be used in later phases of the research. Like all ethnographic research, the meaning of a message is assumed to be reflected in various modes of information exchange, format, rhythm and style, e.g., aural and visual style, as well as in the context of the report itself, and other nuances (p. 68).

Ethnographic content analysis (ECA) involves constant discovery and comparison of situations, settings, styles, images, meanings, and cultural nuances (Altheide, 1987), as a means to check, supplement, and define theoretical claims (Krippendorf, 2013). At its core, a content analysis is a means by which the researcher is able to develop inferences about a phenomenon across any type of communication (Kondracki, Wellman, \& Amundson, 2002). A content analysis involves categorizing and coding raw data, followed by an interpretive or systematic analysis of the data, dependent upon the research questions being posed (Krippendorf, 2013; Hsieh \& Shannon, 2005). This ethnographic analysis type aligns itself with the content and culture to better understand the social systems of the modern world. Specific methodological considerations are chosen based on the cultural forms and practices being analyzed, as a means to provide context to the phenomenon being studied (Hsieh \& Shannon, 2005).

I utilized an inductive ethnographic content analysis as a method to explore how authenticity and social capital was maintained and perceived on YouTube beauty channels. In addition, I sought to understand how branding practice by beauty influencers could affect authenticity and social capital, as perceived by members of the digital public. This was best served by an inductive content analysis method. An inductive content analysis is utilized when existing theory or literature is limited on the phenomenon of interest (Elo \& Kyngäs, 2007; Hsieh $\&$ Shannon, 2005). An inductive content analysis begins with specific data and moves toward the 
general, in an effort to investigate particular instances as being combined into a larger whole (Elo \& Kyngäs, 2007; Krippendorf, 2013).

In an inductive content analysis, categories are derived within the data. Through the process of open coding researchers are able to construct categories from within the data itself (Elo \& Kyngäs, 2007). Open coding begins with making notes and headings within the text while reading it, followed by the generation of rough categories (Elo \& Kyngäs, 2007; Hsieh \& Shannon, 2005). Upon completing open coding, the categories are grouped into higher order headings (Burnard, 1991). Dey (1993) made careful note this is not simply bringing together similar terms. In this phase, data is compared against similar and dissimilar observations to create classifications of belonging to categories (Dey, 1993). The purpose of formulating categories is to describe a phenomenon, by increasing understanding and generating new knowledge (Elo \& Kyngäs, 2007). The abstraction phase involves formulating a general description of the research question through the generation of main categories (Dey, 1993; Elo \& Kyngäs, 2007). Categories are named with context-specific terminology, based on the grouping of subcategories; categories then are combined to form main areas of emphasis addressing the research topic in more generalized terms (Elo \& Kyngäs, 2007).

Digital ethnographic content analysis involves emergent and theoretical sampling of digital documents, development of an analytic protocol, and constant comparisons to clarify themes and frameworks (Altheide, 1987). In regard to this study, authenticity, social capital, branding on social network sites, and communicative practices were not explored for cultural importance to the general act. Instead, these concepts were linked to a course of action as it is reiterated in specific scenarios (Altheide, 1987). The goal of this digital ethnographic content 
analysis was to determine how behaviors, cultural forms, and cultural practices occurred in a specific digital context, through the themes presented by emergent data.

Framework analysis. Framework analysis provides a method of analyzing the data so key themes may emerge, comparing and contrasting data across cases but also within individual cases (Gale, Heath, Cameron, Rashid, \& Redwood, 2013). A framework analysis is not confined to any one specific theoretical approach but rather is a flexible tool that can be adapted for use with many qualitative approaches (Gale et al., 2013). The advantage of using a framework analysis technique is that the researcher can obtain a view of phenomenon as they occur on a case- and theme-level of inquiry (Srivastava \& Thomson, 2009). Researchers are able to analyze meaning on an individual-level through each participant, as well as compare and contrast meaning across cases, to produce trajectories of common themes (Lipstein \& Britto, 2015). It is the proposed homogeneity within and across cases which serves as a basis for framework analysis (Gale et al., 2013; Srivastava \& Thomson, 2009). As authenticity and social capital were assessed subjectively they likely would possess unique case-by-case based attributes. However, cultural dictations of the two constructs also proposed similarities in what is deemed authentic and meaningful (Gilmore \& Pine, 2007). As beauty influencers continued to construct and build their identities on these sites, it was important to assess the process both individually and as a function of the culture growing on social media.

Gale et al. (2013) suggested framework analysis as a systematic tool, capable of being flexible in regard to analysis techniques. Research proposed framework analysis gathers data which is "sifted, charted, and sorted" through a five step process: (1) Familiarization, (2) Identifying a Thematic Framework, (3) Indexing, (4) Charting, and (5) Mapping and Interpretation (Gale et al., 2013; Ritchie \& Spencer, 1994; Srivastava \& Thomson, 2009, p. 75). 
Familiarization occurs when the researcher transcribes qualitative data and becomes familiar with the transcripts (Srivastava \& Thomson, 2009). Here the researcher's goal is to gain an overview of the collected data and become aware of recurrent themes (Ritchie \& Spencer, 1994). Gale et al. (2013) noted a researcher should begin to make marginal notes at this stage, with open coding concluding the familiarization stage (Ritchie \& Spencer, 1994; Srivastava \& Thomson, 2009).

Once the researcher has openly coded the data set, the second stage of framework analysis, identifying a thematic framework, can begin (Srivastava \& Thomson, 2009). The researcher identifies emergent themes, key issues, and concepts which then are used to filter and classify the data into larger categories. Ritchie and Spencer (1994) stressed while framework analysis typically involves data that may address a priori issues, the researcher must maintain an open mind in this stage and not force data into categories. The researcher must work to refine a thematic framework through logical and intuitive thinking, where judgments are made regarding meaning, the relevance of issues, and connections between ideas (Ritchie \& Spencer, 1994). Gale et al. (2013) suggested having an "other" category to code data which does not fit into the categories established in the analytical framework, so as not to ignore data.

The third and fourth stages, indexing and charting, work in sequence to produce thematic matrices. In the indexing stage, the researcher identifies portions of all of the data corresponding to a particular theme (Srivastava \& Thomson, 2009). This process occurs over the entire data set, as opposed to selections of text. Ritchie and Spencer (1994), as well as Gale et al. (2013), suggested using a numerical system to index notions in the text. It also is suggested to use qualitative data analysis tools, such as NVivo, to manage the volume of data and indexes (Gale et al., 2013; Srivastava \& Thomson, 2009). Once all of the data has been indexed, charting can 
proceed, where the indexed pieces of data are charted into matrices (Gale et al., 2013). Charting involves summarizing the data based on categories from each transcript or set of field notes; it is noted good charting requires the careful ability to find balance between reducing the data and maintaining original meaning (Gale et al., 2013). The use of spreadsheets or qualitative data analysis tools can aid in the charting process.

The final stage, mapping and interpretation, requires charted information be displayed in graphics, including charts or matrices (Srivastava \& Thomson, 2009). This phase is often the most recognized in framework analysis, as it provides the schematic diagram of the phenomenon of interest, as a way of guiding interpretation of the data (Lipstein \& Britto, 2015). Miles and Huberman (1994) proposed the creation of matrices can help to extract scenarios cases share, without forcing meaningful sequences. A matrix, as defined by Miles and Huberman (1994), is the intersection of two lists, set up as rows and columns. In framework analysis, a matrix may be mapped for each case or theme, based on the charting process (Gale et al., 2013; Ritchie \& Spencer, 1994; Srivastava \& Thomson, 2009).

From these individual matrices, the grouping of characteristics within cases allows for the creation of partially-ordered matrices, in which characteristics can be viewed across multiplecases (Miles \& Huberman, 1994). Gale et al. (2013) proposed through the proper framework analysis techniques and interpretations "the findings generated through this process can go beyond description of particular cases to explanation of, for example, reasons for the emergence of a phenomena" (p. 117). In the context of this study, framework analysis provided a tool by which subjectivity could be assessed within and across distinct cases, toward the understanding of the phenomenon of interest. 


\section{Procedures}

In this study, I explored the social media platform YouTube as a site of participation, where culture was both built and maintained through communicative elements (Chau, 2010; Jenkins et al., 2006). Through this platform, individual viewers have been able to construct digital identities and produce unique content that other public viewers may watch (Chau, 2010). YouTube, as well as other social media sites, lower the barriers to participate in a given culture, by placing disclosure and authenticity constructs solely on the viewer (Baym, 2010; Ellison et al., 2007). Yet, YouTube is unique in it does not offer the typical connectivity requirements of other social network platforms (Chau, 2010). A viewer does not have to possess a profile on the site to watch videos or connect with content generators. Members typically connect on the platform more informally, often creating a digital public focused on shared interests rather than offline connections. Through this study, I aimed to understand how authenticity, social capital, and branding elements are built and maintained in a digital video environment, utilizing both verbal and contextual clues, YouTube provided the greatest affordances for assessing culture in this aspect.

Case selection. Through this research, I sought to explore how beauty influencers created and distributed messages - involving authenticity, social capital, and branding - as well as how members of the digital public received and categorized these messages. Beauty influencers were selected for this ethnography based on the following parameters: (1) popularity on YouTube, (2) percentage of videos strictly beauty based, (3) video content, (4) frequency of video posting,

(5) inclusion of sponsored, affiliate/free product videos, and non-sponsored videos, and (6) demographics. 
The first parameter for selecting content creators was the relative popularity of the channel on YouTube. I performed a search in December 2016 for channels with the keyword “beauty," which returned 1,160,015 channels. Of those search options, 419 were selected by YouTube for inclusion in the "Beauty and Fashion" category. As YouTube only offers information on a selected number of channels for each category, research into subscriber counts was conducted using the web-based digital analytics platform VidStatsX (2016). VidStatsX (2016) was launched in 2009 and uses subscriber analytic software on YouTube to provide daily subscriber counts for channels, as well as statistics over time. In this study popularity was defined as being a beauty-based channel with at least 2,000,000 subscribers, as this suggests an established channel with an expansive digital public (Caliandro, 2016). VidStatsX (2016) produced a total of 78 "How To and Style" channels with at least 2,000,000 subscribers.

It is noted the VidStatsX (2016) platform includes beauty channels in the category of "How To and Style," which were assessed by the researcher to remove channels which did not post at least $75 \%$ beauty-based content. This was assessed subjectively by the researcher, through inside status as a member of the digital beauty community, through my combing of each channel for video content over the course of one year from December 1, 2015 to December 1, 2016. Lifestyle, do-it-yourself, and home décor videos were the three main content areas that excluded channels from being beauty-focused. This analysis yielded 23 channels based on this parameter.

To further refine the channels for inclusion in this study, I analyzed beauty-content subjectively on each channel, utilizing my stance as the researcher-as-instrument and researcheras-community member to assess the nature of beauty-related content. In the context of this study, any channel promoting theatrical make-up applications for costume where excluded, as they 
represent the entertainment aspect of beauty and do not reflect "day to day" beauty routines.

Additionally, any channel promoting one specific type of beauty practice was excluded. These included channels dedicated to nail art and hair styling. These channels were excluded as they promote a specific sector within the beauty community, whereas this study sough to understand constructs of authenticity, social capital, and branding among beauty influencers in regard to beauty as a whole, rather than focusing on one specific locale. Video content deemed appropriate for this study were tutorials, beauty "hauls", monthly beauty favorites videos, and daily video blogs (vlogs) including at least $30 \%$ of the content involving an aspect of beauty. Based on these parameters for video content, this yielded a reduction to 13 channels from the previous stage.

The fourth parameter focused on the frequency of video uploads. I selected the parameter for this study of at least 52 videos per year, or once a week, based on suggestions that individuals should maintain consistency in sender-receiver message functions (Abidin \& Ots, 2015; Booth \& Matic, 2011). Each of the 13 channels met this parameter. As all channels met this parameter, they were then assessed for their inclusion of content at three levels of monetization, namely sponsored, affiliate/free product, and non-sponsored content, to directly address the main research question of this study.

The parameter of at least five of each type of sponsorship or non-sponsorship throughout the year was selected, as my relationship to the community determined this as a point of saturation. To better understand how YouTube can be used as a source of income generation for professional gains, it was necessary to define the nature of sponsored, affiliate/free product, and non-sponsored videos. As beauty influencers utilize content generation as a source of revenue,

\footnotetext{
${ }^{1}$ Beauty hauls are defined as videos in which beauty influencer show products they purchased or received in gratis to viewers, often as a means of establishing first impressions. They typically explain to viewers why they have selected a product and their expectations regarding its performance, often to establish a baseline of their feelings about the product for further review.
} 
sponsored videos provide a means by which these influencers can create direct revenue through their channels (Wu, 2016). The revenue gained from these videos often is bound by contract, in which the influencer is required to note certain features about a product or brand in exchange for financial gains, typically in the form of direct income. Beyond these stipulations, the sponsorships also had to be beauty-related. For instance, a sponsored apparel video, such as those where the influencer showed viewers how to style apparel products for an event without including a beauty routine, was not included in this parameter. Sponsorship was defined by a Federal Trade Commission (FTC) disclaimer posted in the video's description box, as required when endorsements are made on behalf on a sponsoring advertiser (Federal Trade Commission, 2015).

Another means by which beauty influencers may generate revenue is through affiliate or free product videos. Affiliate videos are defined as those where the creator receives a small percentage of the purchase price for a product, if the viewer uses the influencers' provided link to buy the item. Free-product videos are those in which the influencers receive free products from a brand for review, however, they are not obligated to show the items on screen as it is not directly sponsoring the video's content. In this instance, influencers are receiving economic capital through the acquisition of free products they do not have to purchase themselves, rather than through sales generated by affiliate links. For this study, affiliate and free product videos were included together as both provide economic capital to the influencer through a product, without being directly sponsored by any one brand. Affiliate and free-product videos were selflabeled, as they are not required by the FTC to be noted as such by the beauty influencers in the description box (Federal Trade Commission, 2015). The three most common forms of selflabeling affiliate/free product videos were influencers stating "Affiliate links provided" in the 
description box, noting the video was in affiliation with a brand but not sponsored by it, or through influencer's mentioning products were provided for free by a brand directly in video content.

Non-sponsored videos were those in which the influencer creates content with no sponsored contract agreements, no affiliations, or does not explicitly state products included in the video were received for gratis. Among influencers, the projection is these videos are for the viewer. The content is created to provide the viewer with entertainment, information, or emotional capital. Any unlabeled video was described as non-sponsored content. I selected this parameter as FTC guidelines explicitly state sponsored content must be marked as such and there is an apparent act by influencers to note affiliate content or when products were provided for free. If an influencer does not make note of a sponsorship, an affiliation, or mention the use of free products in the video, it was assumed these categories did not define that content, placing it as being non-sponsored in its entirety. Assessing this parameter excluded four channels, yielding a reduction to nine possible channels for review.

The final parameter assessed was based on demographic information. As this study aimed to address culture as it was built or maintained in a digital context, it was necessary to limit the geographic regions of the beauty influencers to one location, in an effort to reduce the disparities between culturally specific definitions of digital practices. The channels were limited to those where the beauty influencer lived in the United States for at least ten years or longer and was currently a resident of the United States, as these influencers engaged in cultural practices that I understood and prescribed to. I self-identify as a member of the culture of the United States, being a citizen of the country and engage in the cultural practices of this society, I selected these demographic parameters. As I was the instrument in this study, it was essential the culture be 
placed within a context I could distinguish, as not to misinterpret cultural forms or practices in terms of meaning. This reduced the channels to four, of which all were female beauty influencers. Of the four channels remaining, three channels had subscriber counts from $2,000,000$ to $3,000,000$, while the fourth channel had a subscriber count of 5,000,000. I subjectively decided to review the three channels with subscribers in the 2,000,000 category, as the channels showed the greatest consistency in the parameters selected.

Data collection. Data collection occurred in a single phase for each of the three channels selected for digital ethnographic analysis. First I viewed and analyzed the first video posted by each beauty influencer on her respective channel. This served as a reflexive exercise to assess the digital image and identity projected by each beauty influencer. This suggests the type of individual that each influencer wished to project and how that self was defined in a digital context. This also served as a baseline for the projection of authenticity and social capital to the YouTube beauty community, as these identities revealed various mechanisms by which the influencer projected perceived authenticity and engaged with viewers in both bridging and bonding social capital exchange.

Data collection of video posts began with my reviewing each video posted by the three beauty influencers from January 1, 2016 to December 31, 2016. This time period was selected as a means to assess how each construct in this study was maintained or impacted across one year of an established YouTube beauty channel. As this research did not address the creation of these constructs, I wanted to assess how these pre-existing concepts were impacted during a specific time and context. A listing for each video was created, including the video's title, date of upload, viewership statistics, rating ratio (likes/dislikes), and sponsorship status. These lists were created 
for each beauty influencer. This served as an additional measure to assess sponsorship activities with time and provided a basis for selecting individual videos for analysis.

From the bank of videos for an individual beauty influencer, I selected five videos from each sponsorship category, relative to the research questions. Videos were selected based on their viewership statistics, rating ratio, and most commented. Viewership had been suggested as a notion of popularity, proposing videos with the highest number of views were the "most popular" at a given time (Szabo \& Huberman, 2010). Since popularity may change over time, such as the video gaining initial popularity through being featured on the YouTube home page, popularity was assessed by the number of video views on a specific date (Szabo \& Huberman, 2010). Chatzopoulou, Sheng, and Faloutsos (2010) suggested assessing video rating, through comparing likes to dislikes, and reviewing the most commented videos also are means to assessing video popularity among public members. Based on these suggestions (Chatzopoulou, Sheng, \& Faloutsos, 2010; Szabo \& Huberman, 2010), I selected the most and least viewed video in each sponsorship category, the video with the highest and lowest rating ratio, and the most commented video for each influencer. This yielded a total of 15 unique videos, five in each sponsorship category, for each influencer. Additionally, researchers have suggested that both social capital exchange and authenticity are impacted by consistency over time and the type of projections (Burke et al., 2011; Gilmore \& Pine, 2007; Valenzuela et al., 2009), examining these constructs across a popularity continuum helps to assess the reach of the influencer across a variety of contexts. By assessing different contexts for each construct, this illustrated how this research was affected by the qualitative parameters of time and space.

If the videos overlapped in terms of viewership and rating ratio, I selected the video in the category with the next highest or lowest viewership and rating ratio, relative to the metric of 
interest. If the most commented video aligned with one of the other selection criteria, the researcher followed the same protocol as above in selecting the next most commented video that did not overlap another metric. This process was completed for each of the three beauty influencers.

For each of the beauty influencers, I captured the selected videos using the NCapture feature of the qualitative data analysis software NVivo on February 1, 2017. Once the videos were imported to NVivo, I reviewed the videos individually. Field notes were recorded in the video using the coding tool in NVivo, in addition to transcripts being created in Microsoft Word. Coding proceeded from an open stage to a more defined point in the framework analysis, where each frame of the video was addressed to create an index of the content. These indexes then were utilized as a tool to chart categories into interpretive matrices.

In an effort to analyze the textual comments conveyed by viewers, I chose to select the ten most liked comments on each video for analysis. This parameter was selected as researchers proposed that liking a comment or post on a social network site can suggest an implied agreement or connection with the statement by another viewer, without an active need for contribution (Kabadayi \& Price, 2014; Robbins, 2013). Thus, the most liked comments should be those other viewers feel a connection to and are in agreement. Based on this suggestion, the most liked comments are those with the greatest impact, as viewers agree with these assessments by others. Comments were gathered on February 1, 2017 through the use of an Excel spreadsheet. The top ten most liked comments for each video were recorded in their original format, including any Internet lingo or inclusion of emoticons. The metrics for the comments, including number of likes and number of replies, as well as any personal reflexive notes also were recorded on the Excel spreadsheet for data analysis purposes. 
Data analysis. Data was analyzed on a case-level basis for each beauty influencer independently. An entire case (one beauty influencer) was analyzed before moving to the next case. Once each video was imported into NVivo, I began the process of creating digital field notes. The field notes were gathered inductively, however, each section of analysis was guided by classification schemes from the literature.

To assess perceived social capital projected by the beauty influencer, Sander and Lee's (2014a) model served as the initial analysis framework. Sander and Lee (2014a) built upon Narayan and Cassidy's (2001) research on social capital to propose eight dimensions of social capital, as found within social network sites. These concepts are displayed in Figure 3 (below). This model was selected as Sander and Lee's (2014a) focused on aspects of digital social capital, which assessed the ways in which members of a digital community interacted to engage in social capital generation and maintenance. Additionally, this model was one of the few that accounted for differences in social capital between offline and online contexts. As these influencers interact with social capital in an online community, it was necessary to utilize a model which accounted for the variations in bond formation across a digital environment. 


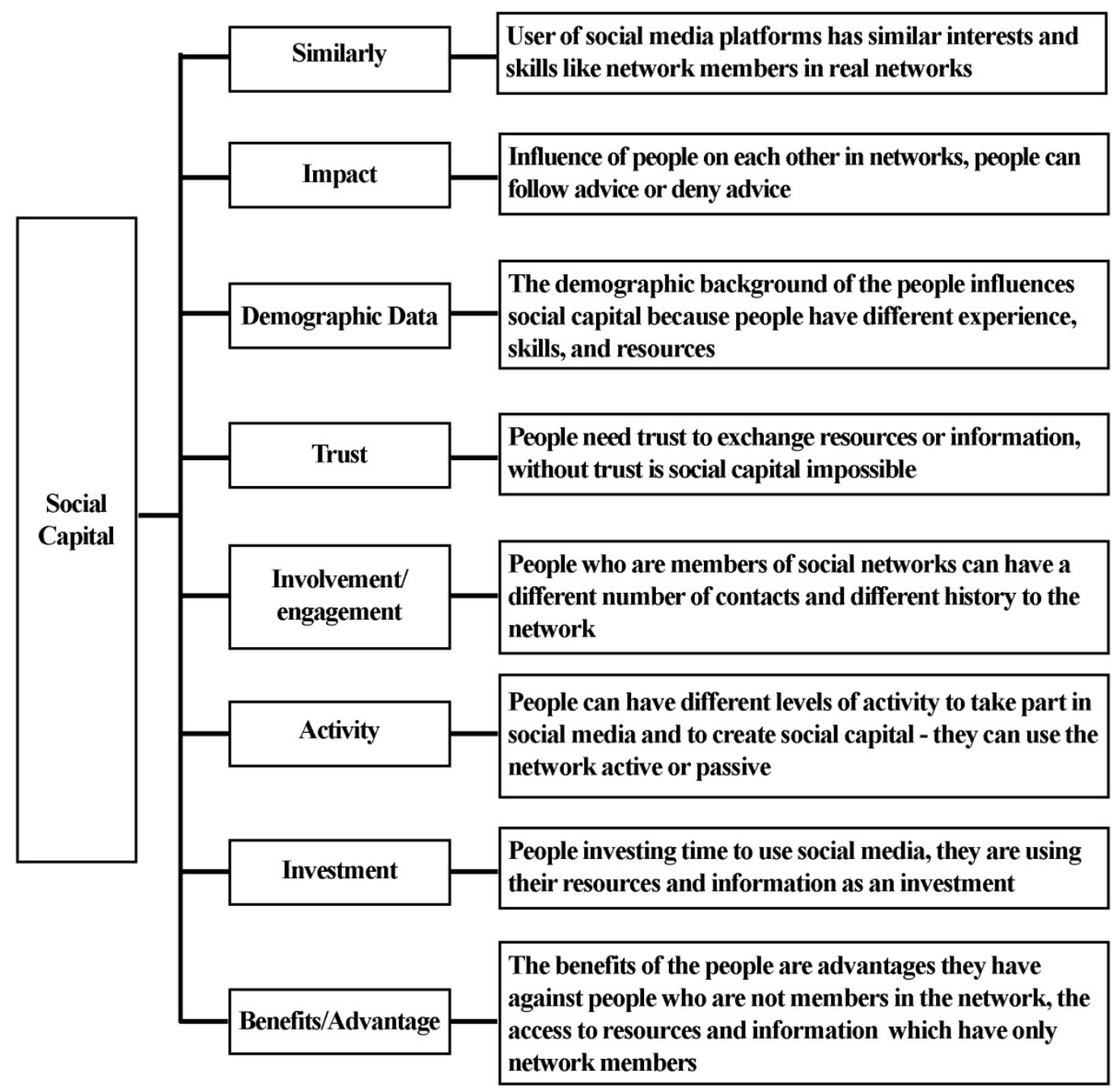

Figure 3. Reproduced model of elements of social network site capital as defined by Sander and Lee (2014a) ${ }^{2}$

The field notes for each video were assessed based on the eight concepts of social capital, as well as an "other" category for those that do not fit into these descriptions. Instances of social capital creation and maintenance were recorded across each sponsored, affiliate/free product, or non-sponsored video. Social capital as perceived by members of the beauty public was assessed through textual analysis of comments. Comments inclusive of establishing or rebutting the

${ }^{2}$ Model reproduced in its original form. Grammatical choices by the original researchers were not modified in this reproduction, to uphold its structure directly from the research it was extracted from. 
notion of social capital between the beauty influencer and viewers served as a point of triangulation for the data.

This study utilized the real/fake matrix as a framework for assessing the types of authenticity and value-placed on chosen types for each beauty influencer across the various types of sponsorship videos, as a means of establishing if or how the beauty influencer crafts and maintains authenticity (see Fig. 2, p. 38). Gilmore and Pine (2007) suggested individuals or companies which have a strong sense of the type of authenticity they wish to project, regardless of whether they appear real or fake, are deemed the most authentic by viewers. Thus, maintaining one type of real and/or fake across a variety of content types, regardless of sponsorship status, will produce the most authentic results. This was assessed by my stance as the instrument, through noting the frequency and impact of deviations from the original projected authentic constructs by influencers, as apparent in their constructed narratives. Deviations were marked according to their polarity in terms of being real or fake, based on Gilmore and Pine's (2007) definitions of the terms and placement within the matrix. In addition to analyzing realfake projections of the beauty influencer as the researcher, authenticity as perceived by members of the beauty public was assessed through textual analysis of comments. Comments inclusive of establishing or rebutting the reality or falsity of the beauty influencer served as a point of triangulation for the data.

Elements of branding were coded inductively, following Aaker's (1996) suggestion of the "brand as person." There was no formal framework for branding utilized, as each beauty influencer produced sponsored and affiliate videos with differing brands, which utilized differing models to build their identity. While there were some similarities in certain brand promotions, each brand carries a unique identity built from within the company and projected through digital 
brand ambassador relationships (de Chernatony, 1997; Senft, 2008). The individual brand identities for each company were not assessed as they contain branding elements unique to each specific company. Instead, branding was assessed as a reflection of the beauty influencers role in the community through sponsored, affiliate, and non-sponsored videos, functioning as an element of brand personality (Aaker, 1996).

Textual analysis of the top ten most liked comments for each video across all three beauty influencers served as a point of triangulation for constructs of social capital, authenticity, and branding. The comments also were assessed for their general reaction to the video content as communicative practices among members of a digital community based on Madden, Ruthven, and McMenemy’s (2012) classification scheme for video comments (see Table 1).

Table 1

Madden et al. 's (2012) classification scheme for video comments

\begin{tabular}{ll}
\hline Category & Subcategories \\
\hline Information & Request \\
& Make comparison \\
& Give (video content) \\
& Give (video context) \\
& Give (general) \\
& \\
Advice & Request \\
& Give \\
& Recommend another video \\
Impression & General positive \\
Negative \\
Opinion & Request \\
& Give (general) \\
& Give (positive) \\
& Give (negative) \\
Give (mixed) \\
Insult \\
Compliment \\
Criticism \\
Tribute \\
\end{tabular}


Speculate

Responses

Agree

Confirm

Disagree

Challenge

Expression of personal feelings

General

Positive

Negative

General conversation

Greetings

Thanking

Joke

Apology

Reference to offsite communication

Status description

Anecdote

Random off-topic exclamation

Request personal information

Give personal information

Express plans/desires/wishes

Anticipate

Site processes

Posting videos

Suggest content for a future video

Request posting of another video

Watching videos

Commenting process

Rating features

Sharing videos

Profiles and subscription

Site policy

Site design

Video content description

Summary (paraphrase)

Summary (direct quote)

Non-response comments

Unclassifiable

Spam

Removed comments

Repetition

Non-English

Nonsense words/punctuation 
This framework was selected as it creates a classification scheme for the type of communication established in comments, removed from the context of the video. These categories focus on elements of interaction in a digital community, as a means of assessing communication practices. This allowed for an assessment of message reception by viewers, based on the projections by the influencer through video content.

Madden, Ruthven, and McMenemy (2012) made note these categories are not mutually exclusive and comments could contain text falling under multiple headings. As the comments section serves as a type of message board for viewers to connect with beauty influencers and each other, it is an important element of participatory culture, in that viewers are able to articulate their interpretations of the messages being sent and generate new content in response to those messages (Jenkins et al., 2006; Madden, Ruthven, \& McMenemy, 2012). Analyzing these comments not only provided a source of triangulation to the qualitative content analysis but also provided a first-hand account of the response to content generation in a digital culture. This helped to define the sender-receiver functions within this digital community. I collected comments for all fifteen of the videos selected for this study by each of the three beauty influencers on February 1, 2017. Any comments added or modified afterward were not considered, as they were outside of the timeframe of this research.

Assessment of quality and rigor. As previously discussed, qualitative methodology typically follows a constructivist paradigm in regard to realities and knowledge generation. The constructivist paradigm positions itself around the idea multiple realities exist, which are bound by individual values (Tashakkori \& Teddlie, 1998). Qualitative research focuses on the epistemological stance that knowledge is a co-creation effort between the researcher and participant, which moves away from a cause-effect relationship, into one grounded in experience 
and individual perceptions of phenomena (Tashakkori \& Teddlie, 1998). In effect, "good" qualitative research understands meaning is constructed personally and acknowledges individual differences can contribute to unique knowledge generation. It is bound by the notions of context and time, where meaning is created through a variety of constructs. Lincoln and Guba (1985) proposed there are four measures used to assess the rigor in qualitative research: credibility, transferability, dependability, and confirmability.

Lincoln and Guba (1985) proposed credibility was a measure of the "truth" in the results. Creswell (1998) later expanded on this view to suggest credibility was a measure of how well the results and analysis measured the knowledge co-created between the researcher and participants. For qualitative results to be credible, they must measure the realities and knowledge generated during data collection as they are understood by the individuals creating that knowledge. As Creswell (1998) suggests, this research utilized triangulation as a measure of credibility. Triangulation involves the utilization of multiple methods, data sources, analysis, or theories to provide a more comprehensive understanding of the phenomena being studied.

In this study, triangulation occurred through multiple types of analysis, as well as through the analysis of viewer-generated comments. The videos were coded utilizing an ethnographic content analysis methodology, which then were subjected to framework analysis of the data. Emergent, reflexive field notes were completed in the initial stage of research. Codes were developed from this research, through framework analysis, which then returned to pure transcripts of the videos to categorize all of the present data. Additionally, an analysis of textual comments on each of the three sponsorship-type videos was conducted utilizing Madden, Ruthven, and McMenemy's (2012) classification scheme. The grouping of comments into larger 
categories and subcategories served as a point of triangulation to the qualitative data, through analyzing the message sent and the perceived response by viewers.

Transferability is the qualitative approach to being able to transfer conclusions from research to other contexts (Lincoln \& Guba, 1985). As the research is bound to context and time, it is typically assumed qualitative research cannot be generalized to a wider population, however, it can be transferable. Lincoln and Guba (1985) noted while it is not true generalization, qualitative research often is descriptive enough to provide a context to the reader, which he or she then may transfer to his or her own interpretation of the phenomena being discussed. As Creswell (1998) suggested, this research utilized thick description, to transport readers and provide a plethora of contextual clues to allow the transfer of knowledge.

Dependability, as assessed by Lincoln and Guba (1985), focused on the consistency of the results. Lincoln and Guba (1985) made the point dependability is rooted in the results being consistent based on the context and time in which they were gathered. This is not a measure of pure reliability, as the bound nature of the qualitative study does not allow for complete replication. Creswell (1998) proposed external audits could provide the means by which to explore the consistency of qualitative results. During an external audit, a researcher or team of researchers secondary to the study assess each element of the research (Creswell, 1998).

For this study, an expert researcher was asked to assess the research on the basis of data collection, analysis, and conclusions, providing an overview of her interpretation of the data. By doing so, the external audit provided a new knowledge base from which the context of the research was explored. Creswell (1998) warned while external audits do risk the issues of conflicting viewpoints or differences in interpretation, they also can provide a rich dialogue across knowledge-bases. As conflicts arose, they were discussed between the auditor and myself, 
upon which final decisions in terms of analysis were made. This external audit helped to assess whether there was a consistency in the results between researchers, as consistent results are deemed dependable.

Confirmability focuses on issues of neutrality in qualitative research (Lincoln \& Guba, 1985). It is a measure of objectivity in qualitative research, where proposing conclusions drawn were the result of participants' knowledge regarding a contextually-bound principle, and not the effect of researcher bias (Lincoln \& Guba, 1985). Creswell (1998) proposed while triangulation can provide one method of assessing confirmability, it ultimately begins assessment through the researcher's reflexivity. It is understood each researcher brings a different background, knowledge base, and skill set when engaging in research, especially so in qualitative research (Creswell, 1998). These preconceptions are not directly reflected as bias, unless the researcher fails to mention them (Creswell, 1998; Lincoln \& Guba, 1985). In being reflexive, the researcher should attempt to remove biases by being explicit in acknowledging and discussing preconceptions do exist. Creswell (1998) suggests that keeping a reflective journal during the research process can help with triangulating the data and also can be a very cathartic experience for the researcher, as it serves as a check of preconceptions. A reflexive journal was completed for each day of data collection and data analysis, and was maintained in a file separate from the ethnographic field notes.

In discussing each of these measures of rigor and quality qualitative research, it is important to address the notion of "meaningful" results. Creswell (1998) proposed meaning is not ascribed to any one part of qualitative research, rather meaningful results are generated through meaningful studies. While this statement may seem to be common sense, Creswell (1998) noted the generation of a meaningful study takes place well before any data is gathered or 
results are analyzed. A meaningful study begins in the conceptualization stages. The scope of the research and the context within which it is explored are central to crafting a study which provides meaning to those both within and outside of the study. Through applying the methods of data collection, analysis, and assessment explored in this chapter to the research questions being posed, meaning was able to be built from conceptualization to actualization.

\section{Ethical Considerations}

The affordances of digital ethnography have posed important considerations regarding ethics in digital research. Differing views of how to maintain privacy, anonymity, and garner informed consent in a mediated environment are current topics of debate (Lange, 2007; McKee \& Porter, 2009; Morey, Bengry-Howell, \& Griffin, 2012; Zimmer, 2010). McKee and Porter (2009) proposed how researchers approach the study may guide ethical considerations. Much of the debate centers on what is considered public information. Zimmer (2010) suggested even though social network sites may be openly-accessible, there is a perceived privacy of the viewer, in that he or she should have protection over when and how information is shared. McKee and Porter (2009) proposed social network data does not constitute interaction with a human subject and is available as existing records, therefore it need not be treated any differently than print medium. Another view by McKee and Porter (2009) placed social media as being a type of community and culture, where ethnographical ethical considerations should be considered. With differing stances in regard to ethics in social network research, it is important to define my stance.

Lange (2007) found what constitutes the public nature of YouTube content is the display of information and content being produced. Some YouTube personalities keep their identities entirely private, posting videos which do not show their faces and refrain from using their 
personal names in video content. In the context of this study, all three beauty influencers used their actual names in their channel name. The beauty influencers freely shared information about the geographic locations in which they live and aspects of their personal lives, including spouses, significant others, children, and close family members. All three of these beauty influencers engaged in a type of video labeled a vlog, where they log activities in their daily lives through video content that are not always related directly to beauty (Miller, 2011). In the context of public and private videos, all three beauty influencers had their videos set to "public," which allowed them to be watched by any viewer, without any requirements of a YouTube account. These beauty influencers appeared to be inviting viewers into their lives and creating a culture through their channels, situating the content and beauty influencers as being "public" in nature (Lange, 2007; McKee \& Porter, 2009).

The American Anthropological Association (AAA) (2016), which governs ethnographic research protocol, states research may be exempt from common rules of review if the research is listed in an exempt category. The AAA lists category four for exemption as "involving the collection or study of existing data, documents, records if these sources are public or information is recorded by the investigator in such a manner the participants cannot be identified, directly or through identifiers linked to the subjects" (2016). In the context of this study, through the actions of the beauty influencers, it was assumed the information being presented to viewers was of a public nature and available through existing data. This research was submitted as exempt by AAA guidelines to the Institutional Review Board. The Institutional Review Board approval is on file for this research.

While this research technically is classified as exempt, following Zimmer's (2010) cautions of maintaining anonymity, the names of the beauty influencers', their channels, and 
comments by viewers were referred to with pseudonyms as necessary. Additionally, no video titles were included in the analysis, to protect the identities of the beauty influencers, as I prescribed to McKee and Porter's (2009) view of the social network site as being a location for cultural development and growth, relative to traditional notions of ethics in ethnographic research that seek to maintain the anonymity of participants in the culture.

\section{Conflict of Interest Statement}

I acknowledge there is no affiliation with or involvement in any organization or entity with financial interest or non-financial interest in the subjects investigated in this research. There were no reciprocal arrangements made with any entities prior to, during, or after this research. As the data for this study was presented on a public social media site with no requirements of participation to review the content, the material is considered exempt by review standards, and the exemption is on file with the Institutional Review Board. The identities of the subjects in this study were protected by pseudonyms to provide a level of anonymity to the beauty influencers. 


\section{CHAPTER IV: THEMATIC INTERPRETATION}

Each beauty influencer in this study had her own unique experiences through her YouTube channel. There were discrete experiences by each beauty influencer which provided insight into the personal and professional affordances of social capital maintenance, authenticity projections, and brand-as-person identity measures, as the influencer defined them. While each influencer was treated as a discrete case, the experiences shared among them served to assess the impact of commoditization of identities and relationship maintenance in a digital culture. To determine how these measures impacted and were guided by the digital culture, it was necessary to understand the individuals which were utilizing this growing digital beauty community as a means of personal and professional growth.

\section{Narrative Development}

This chapter begins with the presentation of influencer identity and cultural environment through digital narratives. These presentations are diverse and highlight the different personal and professional identities of each influencer. The individual presentations were crafted through the narratives of each influencer herself, providing a basis from which she projected an identity and participated in the digital culture created through YouTube. A digital ethnographic content analysis served as the basis for assessing influencer identity. A review of each beauty influencer's channel history prior to January 2016 also was included in the content analysis as a point of assessing demographic data. Videos that were listed as being question and answer or those in which the title directly addressed influencers discussion of personal details, such as "get to know me" or "daily vlog" were reviewed for additional descriptors of demographics.

Ethnographic field notes gathered from the videos served as a framework for locating influencer projected identity and elements of relationship maintenance. 
While there were similarities across the three influencers narratives, each influencer is presented separately to maintain distinctions between how each functioned as an individual and in a professional context. The influencer determined disclosure levels, which may be projected at any level of transparency and honesty (Baym, 2010). These narratives were generated directly from the content projected in influencer's videos and basic statistics from the channel. Therefore, they were subject to the influencer's disclosure levels. This section defines how each influencer created an online image, viewed professional goals, and established personal ties to the community. These narratives functioned as a baseline of the image projected by the influencer to the digital community. As described in the methodology, pseudonyms were used for each influencer and video titles were omitted to obscure the connection between creator and content. The influencers here out are known as Caitlin, Danielle, and Natalie.

Demographic details. Caitlin, Danielle, and Natalie were willing to share personal details about their lives and demographics to viewers. All three influencers were open with viewers about their age, ethnicity, place of birth, current geographic location, and marital status (see Table 2 below).

Table 2 .

Demographic details of Caitlin, Danielle, and Natalie as projected through video content

\begin{tabular}{llll}
\hline \multirow{2}{*}{$\begin{array}{l}\text { Age } \\
\text { Ethnicity }\end{array}$} & Caitlin & Danielle & Natalie \\
\cline { 2 - 4 } & 29 & Approaching 30 & 30 \\
Place of Birth & Mexican, Middle & Mexican & Italian and Argentinian \\
Current Location & California & & \\
Marital Status & Married & Mexico & Miami, FL \\
Children & One son & California & Tampa, FL \\
\hline
\end{tabular}


All three influencers were willing to share personal details with their viewers, allowing them to learn more about their past and engage with them on a deeper level. This information was projected as an element of their friendship with viewers, as friends would know these personal details about each other. Certain parts of this information impacted the content produced by these influencers. For instance, all three influencers noted the warm weather in their current geographic locations necessitated certain needs in their make-up products, such as oil controlling foundations or sweat resistant powder. For Caitlin and Danielle, their role as a mother was mentioned in video content, often referencing quick make-up tutorials for busy women. All three influencers introduced personal information early in their channels history, with Natalie and Danielle offering personal information through question and answer videos during the first year of their YouTube channel. This openness suggested influencers saw this demographic information as being a way to open themselves up to viewers, with the potential to gain deeper bonders.

In the following sections, each influencer is discussed individually in regard to her professional status as a content generator. I explore the nature of influencer content generation through frequency of sponsored, affiliate/free product, and non-sponsored videos. I then explore the concept of image and relationship development by each influencer. In this section, additional elements of image creation, identity projection, and relationship development will be included on a case-by-case basis, as each influencer differs in levels of disclosure.

Caitlin - Identity creation and maintenance. Caitlin posted her first YouTube video in September 2009. The video received 42,620 views as of April 2017. The video opened with animation that spun around to read "how to have bigger and fuller lips" in pink letters on a white background. Caitlin was sitting in front of a camera and taught viewers how to make their lips 
look fuller by applying lipstick in a specific way. Near the beginning of the tutorial, the text "This was my very first video!!.. yiikees"3 appeared on the screen. Text was displayed on the screen explaining each step as Caitlin demonstrates. She leans in to the camera to show viewers each step, pointing to the colors she was using from palettes or showing individual products for color reference. In the description bar, she also included tips for viewers such as "Light colors will make any part of your face bigger" or "contouring darker shades of color make your face smaller!!”

The video did not feature any verbal discussion but music was included. In a later video, Caitlin remarked the original music was removed for copyright violation and was replaced with generic clips from YouTube. The video appeared to be shot in a single clip, however, transitions were added to show Caitlin's face fading in and out of frame with each new step in the tutorial. At the very end of the video, text was displayed on the screen reading "Hope I could help.. remember.. rate comment $\& \&$ subscribe!!" The video concluded with a screen showing her name and read "Your official make up artist." After posting the video in 2009, Caitlin did not post another video until October 2012, which was a Halloween make-up tutorial. Caitlin began posting videos on a more frequent schedule in January 2014, at which point she posted an average of one video per week. This continued up to the point of this study.

Caitlin mentioned in an April 2015 question and answer video she was a "full-time beauty and fashion [influencer]." In the same video, she noted she "started [her] channel in 2009, just to comment and like on other people's videos." She watched tutorials to learn techniques for applying her own make-up. After a period, Caitlin explained she "gained the confidence to

\footnotetext{
${ }^{3}$ The comments presented by viewers have been reproduced in their original format, including any Internet-specific text, linguistics, or notations. Notion of [sic] will not be included as Internet speak may follow unconventional standards of grammar or spelling, which are generally understood among digital community members.
} 
finally put a video out because I wanted to see what response I'd get." She stated while she was a beginner at make-up, she wanted others to "feel beautiful." She stated, "Now I have a love for it. I just love the whole thing about YouTube. I feel like it's an art within itself. So, I love it! And now I'm here doing videos for you guys!"

For the time period included in this study, Caitlin posted 61 videos from January 2016 December 2016. Of those 61 videos, 57 were beauty related, contributing to $93.4 \%$ of the channel content. Caitlin saw a tremendous growth in her channel even within the past year. Of her top ten most viewed videos on her channel, seven were posted within the past year. Part of her channel's major growth in the past year could be attributed to an August 2016 video which garnered 5,798,999 views as of December 2016. This video ranked as the most viewed video across the three influencers, over five times more viewed than any video posted by Danielle or Natalie. With this large viewership statistic, it was likely Caitlin gained additional exposure to members of the beauty community through this single video content, even though it appeared to have gained this through its viral nature. As of December 2016, Caitlin had over 2.1 million subscribers and over 54 million video views on her beauty channel. As Caitlin's channel grew, she transitioned to being a full-time beauty influencer and now gains revenue from YouTube directly, through platform-related monetization and partnerships.

As with all three beauty influencers in this study, Caitlin gained revenue through sponsored, affiliate/free product, and non-sponsored video content (see Table 3 below). Of all three categories, the non-sponsored videos received 2.76 times more views than sponsored content and 1.11 times more views than affiliate/free-product videos. Overall, non-sponsored content on Caitlin's channel received 19,824,919 views, which was the highest of all three influencers in this study. Across all three categories, the sponsored videos received the highest 
like/dislike ratio, at 61.05 likes per one dislike. Caitlin was the only influencer who had the highest like/dislike ratio with her sponsored video content, which was nearly six times higher than Danielle's sponsored ratio and three times higher than Natalie's ratio for sponsored content. The non-sponsored content was over eight points lower at a ratio of 53.26, while the affiliate/free product videos produced the lowest ratio of 50.32. These statistics suggest while viewers watched non-sponsored and affiliate/free product content more frequently, they had a preference to like the sponsored content better.

Table 3 .

Sponsored, affiliate/free product, and non-sponsored video statistics for Caitlin's beautyrelated video content posted January - December $2016^{*}$

\begin{tabular}{lcccc}
\hline & Videos Posted & $\%$ of Uploads & Total Views & Total Like/Dislike Ratio \\
\cline { 2 - 5 } Sponsored & 15 & $26.32 \%$ & $3,601,016$ & 61.05 \\
$\begin{array}{l}\text { Affiliate/Free } \\
\text { Product }\end{array}$ & 15 & $26.32 \%$ & $9,898,002$ & 50.32 \\
Non-Sponsored & 27 & $47.37 \%$ & $19,824,919$ & 53.26 \\
\hline
\end{tabular}

Note. Statistics for individual videos were gathered on February 1, 2017.

Caitlin's videos maintained a consistency in production quality across the content reviewed for this study. Caitlin utilized both talk-through and voice-over content in her make-up tutorials. Talk-through tutorials were those in which influencers instruct viewers on how to use products or highlights product features in a real-time fashion, where they are telling viewers as they show them. Voice-over is a production technique in which influencers lay their voice over content being shown on the screen. In her voice-over tutorials, Caitlin did not move her mouth, making it apparent she was not speaking during the actual make-up application. Instead, Caitlin's voice was edited into the video at appropriate times to provide verbal discussion of content when necessary. In some videos, Caitlin blended the talk-through and voice-over content together. 
Both production techniques generated a different tone to the videos. The talk-through tutorials appeared more informal and often showed elements of Caitlin's personality, while the voiceover's were informative in nature and focused on production quality. As compared to the other two influencers in this study, the switch in content type between talk-through and voice-over content was not as apparent in Caitlin's videos; it was very seamless, with the voice-over adding new information without seeming out of place in the overall tone of the video. The integration of both techniques produced content varied enough to be entertaining and engaging, yet structured in a way to provide specific information in a clear format.

While the production features used in Caitlin's videos helped to provide engaging, informative content through visual, auditory, and textual cues, Caitlin also has utilized her channel as way to create and project a digital image to attract viewers to her content. Of all three influencers in this study, Caitlin was the most light-hearted in her content. Her videos rarely opened with her introducing the content, but rather showed a humorous or personal moment to the viewers. For example, in an August 2016 affiliate video, Caitlin showed a clip of herself lipsyncing to popular music with a make-up brush, prior to explaining any video content. In her most viewed non-sponsored video from June 2016, Caitlin opened with saying, "I'm just going to have a Cheetos break," which followed with her showing a bag of Cheetos and eating one on camera. During a make-up tutorial in another June 2016 video, Caitlin told viewers she was going to curl her eyelashes, then proceeded to pick up the eyelash curler and said, "I'm going to go ahead and curl my lashes, using this Tarte eyelash curler that has a ton of mascara on it. So, I'm just going to wipe it off real quick." Viewers could see the product had mascara on it and not in pristine condition, as often was projected by the other two influencers. She did not focus on creating an image of perfection but one of relatability. 
Caitlin's channel saw a large growth during the time period in this study, gaining nearly one million subscribers in 2016 (Socialblade, 2017). Caitlin projected an image of relatability through her humor and honesty. She stayed connected with viewers through YouTube commenting features and other social network platforms, allowing her to communicate with them beyond the video content. Caitlin utilized her content as a way to attract viewers, then her personality to retain them, building on her human characteristics as a source of similarity with viewers.

Danielle - Identity creation and maintenance. Danielle posted her first YouTube video in July 2008. The video had 166,047 views as of April 2017. The video opened with the camera shaking and a view of the ceiling. Danielle turned the camera around and addressed viewers with "Hey everyone." She showed viewers her custom eyeshadow palette and suggested viewers can "take this look from daytime to nighttime by just adding one simple color [points to a navy blue shade in the palette], this one right here." She told viewers the eyeshadows were sold separately so the viewer could customize the shadows to his or her liking, selecting shades to complement his or her skin tone. She described the mineral eyeshadows as "the bomb," since they are excellent quality, in her opinion. She noted she was going to "do a close-up" and "it's gonna be kind of freaky cause I'm going to be all up in the camera." She sat down the eyeshadow palette and leaned in to show viewers a closer look of the eyeshadow. Danielle also told viewers she had filmed this video once before but the "camera cut her short" and she deleted the footage. She ended the video by saying, “...but as long as I make a video, I'm happy!”

Danielle's first video featured no editing and was filmed in a single clip. There was no music, as Danielle engaged in a talk-through for the duration of the video. In the description bar, a link was included to her personal beauty consultant website so viewers could purchase products 
from the video. In contrast to Caitlin, Danielle's channel took a different path following her first video. In her first year of creating YouTube content, from 2008 to 2009, Danielle posted 219 videos, for an average of 4.21 videos per week. Most of her videos were beauty related, focusing on hair or make-up tutorials. This continued throughout the first few years of her channel, with uploading frequency varying based on her work and family situations, which she discussed in videos.

Danielle was very open about her life before being a YouTube influencer. Danielle served in the military for five years, three in active duty and two on reserve, which she frequently discussed on her channel. She mentioned in previous videos it was her career in the military as a mechanic which prompted her to begin YouTube. In an early video from her channel, posted May 2009, Danielle noted the reason she began YouTube, stating, I just started cause I got inspired by a lot of girls on YouTube making videos and I was like, you know, I would work as a mechanic and then I would, I would be working as like a manly job all day. So, then I would get home and just YouTube would make me feel like really girly.

She now generates revenue from being a beauty influencer through sponsorships and opportunities from her YouTube career, such as being on the cover of Parents Latina magazine.

During the time this study occurred, Danielle's uploading frequency declined drastically from her initial schedule. From January - December 2016, Danielle posted a total of 75 videos. Of those uploads, 53 contained beauty-related content, accounting for $70.7 \%$ of the video content on her channel. As of December 2016, Danielle's channel had reached over 2.1 million subscribers, with the channel gaining an average of 10,000 new subscribers monthly. There was no tremendous growth of the channel in 2016, as opposed to Caitlin's channel. However, Danielle did appear to have a consistent number of new subscribers and video views gained each month. 
As with the other two influencers in this study, Danielle produced sponsored, affiliate/free product, and non-sponsored content for her channel (see Table 4 below). Of the three categories, the affiliate or free product videos generated the highest number of views and the highest like/dislike ratio, at 3,426,213 and 27.61 respectively. Non-sponsored content was only slightly lower at 3,408,403 views and a 24.85 overall like/dislike ratio for the category. The sponsored content for the year produced lower total views and like/dislike ratios. Even though there were only four fewer sponsored videos than those in the affiliate/free product category, the sponsored videos produced an overall like/dislike ratio of 13.67 , over 14 points lower than the affiliate/free product content. The sponsored videos only received 1,823,400 overall views, nearly half as many as the other two categories, suggesting viewer preference for non-sponsored or affiliate/free product content.

Table 4.

Sponsored, affiliate/free product, and non-sponsored video statistics for Danielle's beautyrelated video content posted January - December $2016^{*}$

\begin{tabular}{lcccc}
\hline & Videos Posted & $\%$ of Uploads & Total Views & Total Like/Dislike Ratio \\
\cline { 2 - 5 } Sponsored & 13 & $24.53 \%$ & $1,823,400$ & 13.67 \\
$\begin{array}{l}\text { Affiliate/Free } \\
\text { Product }\end{array}$ & 17 & $32.08 \%$ & $3,426,213$ & 27.61 \\
Non-Sponsored & 23 & $43.39 \%$ & $3,408,403$ & 24.85 \\
\hline
\end{tabular}

Note. Statistics for individual videos were gathered on February 1, 2017.

On Danielle's channel, there were very few differences in the production and editing techniques used between the three video categories. Danielle's content was extremely professional in quality, partially through the editing techniques she employed. Her videos did not feature loud music which might distort her voice, nor were there any harsh transitions between frames. Danielle began most of her videos sitting directly in front of the camera, with a 
background complimenting the content of the video or time period in which it was filmed, such as a decorated tree during the winter holiday season. When creating make-up tutorials, Danielle often zoomed in on the area of her face she was focusing on, to provide the viewers a better look at the technique or product she was using. Her content was edited in a way that focused on providing viewers with information, rather than displaying quirks in her personality. However, this did not assume Danielle did not wish to connect with viewers on a more personal level, as she achieved this through variations in her talk-through and voice-over content. In regard to her editing techniques and viewer preferences, she remarked in a September 2016 video,

I know you guys really enjoy doing, the talking videos a lot more, but I really love filming make-up tutorials and I love make-up, um, but it just seems that you guys really enjoy the talking videos. So I will do a mix of everything I love on this channel.

The talk-through portions often opened and closed each video. She personally welcomed viewers to her channel and gave a brief description of what the video would contain. When the video changed to the tutorial or main content, Danielle typically utilized a voice-over to explain each step without compromising the quality of the visual on the screen. In Danielle's videos, there was a clear division in terms of what the video was meant to portray in each section. The talk-through portions were personal and emotionally driven, yet, not as light-hearted as Caitlin's content. Danielle asked viewers for their opinions on topics, such as in a June 2016 video where she showed new make-up products to viewers and frequently asked them to comment down below if they would like to see reviews on them. In the voice-over sections, her videos were much more informative and technical in nature. She gave direct advice to viewers, such as in a make-up tutorial when she told viewers to use a specific brush to apply eyeshadow for a softer look, and demonstrated the technique. During this section, she zoomed in on her eye and to show the brush technique. The voice-over allowed her to gather her thoughts and gave coherent 
directions to her viewers. This combination of techniques allowed Danielle to shift between providing emotional and informative elements to her viewers.

The display of emotions in a professional context was a hallmark of Danielle's channel. She did not mind sharing personal details with her viewers, such as her journey to adoption or political views, however, she did so more formally than the other two influencers in this study. Her channel had a more serious tone than the other two, focused heavily on the content and less on her personality traits. She did not eat Cheetos on screen or make jokes about her dance moves, as Caitlin did. Danielle provided more knowledge-driven content and trusted her viewers were aware of her channel's purpose, without added humorous or candid aspects.

Danielle made this even more apparent in her interactions with viewers. Danielle asked for viewer opinions which were related directly to her beauty content. In one video, Danielle requested viewers to comment "if you have tested one of these self-tanners" and stated she "would love to hear your feedback" about the specific tanning products she showed in the video. In another instance, she told viewers "And if you guys see anything that you really like or a specific swatch video, you guys have to request it or else I don't know if you really want to see it," implying she would like her viewers' feedback in order to produce content they desired. She did not directly respond to viewers' comments as frequently as Caitlin, but when she did, it was in regard to video content or answers to her prompts. Her discourse with viewers focused on how she could make her videos better suited for the beauty community she had situated herself within.

Overall, Danielle created a channel with a very clear focus. She provided content in a coherent, professional, easy to replicate format, which she achieved through her filming and editing techniques. While Danielle's content was very professional and focused on being high 
quality, she remained very open with her viewers, sharing personal details from her life in addition to her beauty content, generating a community focused on her life as a whole, not just the beauty world.

Natalie - Identity creation and maintenance. Natalie posted her first YouTube video in May 2010. The video received 242,167 views as of April 2017. The video opened with Natalie centered in the screen. She appeared to be sitting in a bedroom based on the visible furniture. She opened with "Hi YouTube! I'm back," and waves at the camera. She then asked viewers to "excuse the same top" as "it's the same day. I'm doing the same two videos, one day." It was noted this was the first video posted to Natalie's channel as assessed in April 2017, however, she addressed viewers as if there were videos posted prior to this. A review of her channel and online video statistics did not produce any earlier content, so it was assumed for this study this was the first video posted to YouTube from her account. She then told viewers this was a "better quality video," insinuating she had filmed content before, possibly on another video sharing platform. The content of this video focused on her favorite drugstore make-up brands. She explained how she enjoyed this type of video content the most, as she personally utilized review videos on YouTube to gather information about products prior to purchasing. She told viewers she "is hoping that since the reviews have helped me, then maybe some of my reviews, will help you." She then proceeded to show viewers drugstore make-up products and explained why they were her favorites. She frequently mentioned products were her favorites because "of YouTube," in addition to noting their color selection or similarity to higher-end products were the main reasons these specific drugstore products were her favorites.

Throughout this first video, she also disclosed personal information to her viewers, such as telling them she traveled between her home and her boyfriend's home frequently, keeping her 
make-up products in a travel bag, which was why the packaging was dirty on the outside. She closed the video by telling viewers, "So, video number two. Whoo! Go me!" As this was the first video officially posted to her account at the time of this study, this statement suggested Natalie had filmed a video prior to this and either deleted the content or chose not to upload it. There was no mention of this earlier content in later videos. She ended the video by telling her viewers,

Thank you so much again. Rate, comment, subscribe. Again, if you want to see any videos, if you have any ideas, if you want me to do anything for you, just let me know. I'd me more than happy to! Okay, thanks guys, bye.

Of the three influencers in this study, Natalie was the only one to verbally ask viewers to subscribe, rate, or comment on her videos with this initial content. Natalie also was the only influencer to include a FTC disclaimer in the description box stating this first video was not sponsored and all products were purchased by her. As this was her first video, this statement may have been added as her channel grew, however, she was the only influencer in this study to begin adding FTC disclaimers early in her video content. As with Caitlin and Danielle, this initial content posted by Natalie was composed of a single camera shot. There was no editing to the video and noise from a television could be heard in the background for the duration of the video. Natalie frequently looked away from the camera when describing products and appeared to be more nervous than the other two influencers, often fixing her hair or scratching at her arm. Compared to Caitlin and Danielle's first videos, Natalie's video was the longest, at 9:48, and contained the broadest amount of information to viewers, both beauty-related and personal content.

After posting this video, Natalie continued to post regularly to her channel, uploading 79 videos from May 2010 - May 2011. Of the three influencers, Natalie continued with the most consistent uploading over the history of her channel, posting 85 videos her second year and 67 
videos in her third. Over the past seven years, Natalie maintained a consistent upload schedule of 1.06 to 1.63 videos per week on her beauty channel.

Natalie mentioned in several videos how YouTube was her career and she was grateful for the opportunities she has been provided through being an influencer. She often thanked her viewers for their support and promised to create new content for them on a regular basis. In a question and answer video from 2013, she discussed the reason she began making YouTube videos, stating,

I started YouTube because I had always had a natural talent for hair and make-up. And I was watching somebody on YouTube and I saw all these girls showing their tips and their tricks and their tutorials and stuff like that, and I was just kind of like, 'Hey, I can do this too.' You know?... So, I started YouTube because I just wanted to share my knowledge with you guys and connect with you and build online relationships, which I have done. So, I'm so thankful and grateful for all of you for supporting me...

In an April 2016 video, Natalie mentioned she still loves YouTube and sees herself continuing to create content even though she was "venturing off and doing other things besides just doing like one YouTube thing." She stated in this video she did not discuss partnerships or activities outside of YouTube until the appropriate time, which often made her appear as though she was not actively creating content for her viewers, however, she tells viewers this was not true. She often ascertained the reason she waited to share items was to ensure the information was correct, so viewers could trust information being shared, especially through partnerships or sponsored content.

During the time in which this study occurred Natalie uploaded 55 videos, of which 42 were directly beauty-related, accounting for $76.36 \%$ of the content on her channel. However, when there was a lapse in her uploading schedule, she explained to viewers in a video why her content was delayed. For example, in a video from April 2016, Natalie explained her lack of uploading and promised to produce content more often by stating, 
So if you don't follow me on Twitter or SnapChat, you're probably like, 'Where the eff has Natalie been? Where's she at? She just leaves us, she just disappears on us.' I'm here today to fill you in... we have purchased a home! Whoo!... I can't wait to show you guys the house and everything we do to it. I am going to be doing a lot of vlogging, while I am kind of away. I only plan to take off kind of two weeks, to get this whole thing done.

Even with larger breaks between producing content in current years, Natalie had the highest subscriber count across the three influencers in this study, totaling over 2.7 million subscribers in December 2016. Natalie's channel saw its greatest growth two-to-three years ago, however, it still produced an average of 10,000 new subscribers each month during the duration of this study. This plateau in growth also could be seen in her most popular videos. Of her top ten most popular videos, none were filmed in 2016. Her most popular video was filmed over five years ago in March 2012, with many of the popular videos being filmed at least three years ago. While her channel did not see tremendous growth in the past year, Natalie's channel still produced the highest total subscriber count and highest like/dislike ratio rating (154.90) among the three influencers, suggesting her plateau in growth did not greatly influence her overall impact and connection with viewers.

Similar to Caitlin and Danielle, Natalie generated revenue from her YouTube content. Natalie participated in producing sponsored, affiliate/free product, and non-sponsored content for her channel (see Table 5 below). Of these three categories, affiliate/free product videos generated the highest total views, at 9,654,246. However, the non-sponsored category generated the highest like/dislike ratio at 62.66. Natalie's channel also produced the highest like/dislike ratio of the entire study in an October 2016 video which generated a ratio of 154.90. Natalie's nonsponsored content produced some of the highest like/dislike ratios of the entire study, with most of her non-sponsored content producing ratios over 50 . The affiliate/free product videos produced a ratio of 46.21, 16 points lower the non-sponsored content. While viewers did watch 
the affiliate/free-product videos more often, they showed a preference to like the content in nonsponsored videos. In comparison to the other two categories, the sponsored content on Natalie's channel produced the lowest total views at 6,715,282 and overall like/dislike ratio at 22.92.

These videos generated two-to-three times less views than the other two categories, even though the total number of videos produced for the three categories was nearly even. In terms of like/dislike ratio, the sponsored content produced a ratio of just one-third of non-sponsored content and half of the overall ratio for affiliate/free product videos. These statistics suggested viewers had a preference to view and like non-sponsored and affiliate/free product content much more than content in the sponsored category.

Table 5 .

Sponsored, affiliate/free product, and non-sponsored video statistics for Natalie's beautyrelated video content posted January - December 2016*

\begin{tabular}{lcccc}
\hline & Videos Posted & $\%$ of Uploads & Total Views & Total Like/Dislike Ratio \\
\cline { 2 - 5 } Sponsored & 12 & $28.57 \%$ & $6,615,282$ & 22.92 \\
$\begin{array}{l}\text { Affiliate/Free } \\
\text { Product }\end{array}$ & 16 & $38.10 \%$ & $9,654,246$ & 46.21 \\
Non-Sponsored & 14 & $33.33 \%$ & $8,739,472$ & 62.66 \\
\hline
\end{tabular}

Note. Statistics for individual videos were gathered on February 1, 2017.

Throughout all three sponsorship categories, Natalie utilized a variety of editing and production techniques to engage viewers. Natalie did not use a single static background, nor did she always appear in the same location in her home. When she did use a static background, she changed the color or pattern to match the mood of the video. For instance, in a January 2016 affiliate video, she appeared in front of a purple background to show viewers an "easy fresh \& fast" makeup tutorial. Just a week later, her video was filmed in front of a metallic, glitter-filled silver background to demonstrate a "bombshell" makeup look. The two background choices added to the tone of each video, the purple being softer and more natural, while the metallic 
background added an element of glamour to an extravagant makeup look. Natalie's backgrounds served as a compliment to the content, helping to create a unique overall theme, which then was supplemented by additional editing techniques.

In regard to the other two influencers in this study, Natalie's editing was considered less theatrical than Caitlin's and less professional than Danielle's, overall being described by viewers as “down-to-earth." For instance, Natalie did not change camera angles to show her applying products, as Danielle did in her videos, nor did she use editing to add comedic relief to her content, as Caitlin typically did. Instead, Natalie used her editing to add information or correct content post-filming. In a September 2016 sponsored makeup tutorial, Natalie included a bar on the side of the screen displaying textual information about the products being used. In this sidebar, she included facts about the products for viewers, such as "formula features Japanese wild rose to visibly tighten pores after 28 days of regular use." This information bar allowed her to provide additional facts about the products after filming so she could focus on demonstrating the product in the video.

Similar to how she used editing to provide additional information, Natalie utilized talkthrough versus voice-over in a similar fashion. Her talk-through tutorials typically were much longer and more personal, providing viewers with information but focusing more on the idea two friends were having a conversation. For instance, in a January 2016 talk-through tutorial, she told viewers she was going to "wing it." She reminded viewers throughout the video she "wasn't sure what [she] was really doing," in terms of a final look and she "wanted to film" in order that she and the viewers would "come up with something" together. She shared personal stories during the video and focused more heavily on telling viewers her tips and tricks for applying make-up, rather than giving product descriptions or reviews. In contrast, her voice-over content often was 
much shorter and more centered on providing specific information. Most of her videos utilized voice-over content to interject information throughout talk-through tutorials, adding extra information she had deemed relevant after filming her initial thoughts. When a voice-over was used for the entirety of a video, the content was very specific and focused on an end-goal, such as in two Halloween tutorials posted to her channel. These videos were meant to produce a final product easily replicated by viewers, which required more thorough step-by-step instructional content through a planned and scripted voice-over.

Of the three influencers, Natalie was the least likely to interact directly with viewers in a digital context. She often would ask viewers for their opinions on a look or to share their recreations with her, however, she did not directly interact with viewers in the comments section. She did make note in several videos that she tended to interact with viewers more on her other social media platforms, such as Instagram and Twitter, explaining in more detail in a January 2016 video,

So I hope you guys try it out. If you do, tag me on Instagram or tweet it to me. If you tweet it to me, I'll most likely see it. On Instagram I get tagged in so many things... So if you want me to see your recreations, tweet them to me and I will probably follow you, because I like to follow all of you guys.

In addition to connecting with her viewers on other social media platforms, Natalie also was the only influencer to offer time for viewers to meet her in person, through planned meetups with companies. During the duration of this study, Natalie traveled to Miami, New Jersey, California, and New York City to meet her viewers in person, through opportunities with brands with whom she had partnered. She would tell viewers the date and time of the meet-ups, mentioning the information in videos both verbally and in text several times leading up to the meetings. In March 2016, she posted a video about her meet-up in New Jersey, showing her interacting with viewers, hugging them and taking pictures together. 
One viewer described Natalie as being the "original" YouTube influencer. Her realistic personality and informative video content garnered her over two and a half million subscribers, the highest of all three influencers in this study. Natalie was open with her viewers, telling them she did have life events happening outside of YouTube and promising a brief break in content did not mean she was leaving the channel permanently. Natalie constructed an identity of a relatable, realistic woman who just loved beauty.

Summary of identity creation. In this section, personal narratives for the three beauty influencers included in this study were presented as individual cases. These narratives were depictions of the image and identity each influencer crafted and displayed through her YouTube channel and the digital beauty community. Each influencer functioned as a discrete, unique case. The mechanisms and identity-building tools utilized by each influencer were further defined by her personal disclosure, which then was assessed individually by each viewer. These narratives serve as an integral point in understanding not just who these three influencers say they are but how they have become this person.

Social network sites are both heralded and rebuked for their ability to place control back into the hands of the viewers (Baym, 2010). These sites exist in an action-based environment, where influencers can construct an identity and interact with viewers as they deem necessary (Cova \& White, 2010; Ellison, Steinfield, \& Lampe, 2007). The remainder of this chapter seeks to explore the concepts of social capital, authenticity, and personal-corporate brand identity measures as a means of assessing how the discrete experiences and identities formed by these three influencers impact upon and are guided by the digital environment, through a person-asbrand lens. 


\section{Social Capital and Beauty Influencers}

Each beauty influencer in this study had her own unique experiences with the processes of image creation and identity formation, which ultimately affected her perceptions of social capital, authenticity, and brand relationships. There are, however, similarities in experiences and processes among these three beauty influencers which provided insight into a larger discussion of YouTube as a source of generating tangible and intangible resources. While each influencer still enacted personal measures to foster social capital, garner authenticity, and position themselves relative to brands, the similarities in these concepts shared among the three women provided a context for understanding their function in a larger digital environment.

The results emanating from examination of the similarities and differences between each influencer through her personal narrative are discussed across four thematic areas: (1) Social capital generation and maintenance, (2) Authenticity of the person-as-brand, (3) Brand relationships and the person-as-brand, and (4) Message reception through digital communication channels.

Social capital maintenance is defined as the components of image, identity, and experiences projected by the influencer toward providing informational benefits (bridging capital) or personal, emotional benefits (bonding capital) (Putnam, 2000). This section revisits Sander and Lee's (2014a) model of digital social capital measures to assess these YouTube beauty channels as digital environments, with the influencer serving as a generator and moderator of digital social capital and the viewers serving as receivers and assessors of these measures. Amendments to the model based on the results of this research were proposed.

As these three influencers utilized YouTube as a source of revenue, generating tangible resources for the influencers, this positioned them as a type of enterprise, providing a service to 
viewers in exchange for resources. These influencers function as a person-as-brand, utilizing independent authenticity factors to create an identity meeting the needs of varying target audiences. As such, Gilmore and Pine's (2007) model was applicable to their person-as-brand status, suggesting deviations could construe measures of reality and cause the influencer to be seen as less authentic. The construction of authenticity by these three influencers was assessed in the second thematic area, with deviations from the self serving as an indicator of real or fake authenticity activities.

A third thematic area was defined by each influencer's status as an agent of branding through Aaker's (1996) model of brand identity. Aaker's (1996) model would situate the influencers as part of the brand as person category, providing personality measures for the brand and helping to generate brand-customer relationships. How influencers project brand identity measures for corporate beauty companies was explored in this section.

The fourth, and final, thematic area assessed the communication channels presented between influencers and viewers through the commenting feature provided by YouTube. The projections of perceived social capital, authenticity, and branding measures by the influencer were assessed by viewers through commenting capabilities, which provided an initial level of understanding for a feedback loop in the YouTube digital community. This section served as a presentation of viewer comments as they related directly to the communicative and social aspects of the beauty community at large, as individual viewer comments were presented in each segment as a point of discussion and triangulation.

These four thematic areas are not presented as unrelated concepts. Each thematic area was part of a larger whole, which helped to define the context within which YouTube beauty influencers impact upon and were impacted by the digital environment. Factors from one 
thematic area could relate to measures in other areas. For instance, social capital could be influenced by each viewer's subjective analysis of influencer authenticity, which could be impacted by deviations from the projected self. These concepts are fluid and function as one piece of a larger area of interpretation, toward understanding how YouTube beauty influencers were situated within this digital environment.

This study suggests a new conceptualization of Sander and Lee's (2014a) model of digital social capital, as it relates to YouTube beauty influencers. Traditionally, definitions of digital social capital focus on the concept of interpersonal relationships and how digital media is utilized to gain bridging and bonding capital between two individuals (Burke et al., 2011; Ellison et al., 2011; Sander \& Lee, 2014a, 2014b; Valenzuela et al., 2009). However, less attention has been paid to assessing how social capital was maintained in well-established online communities, such as those formed on YouTube, specifically when social capital was generated on a one-to-many scale. An additional layer of interest was added when considering the monetization affordances of content on YouTube, whereby digital influencers earn tangible, monetary resources for producing content once they reach a certain threshold of subscribers and viewership statistics (Freeman \& Chapman, 2007; Wu, 2016). As beauty influencers on YouTube continue to gain notoriety with viewers and garner additional monetary resources from their viewers' support, it was important to understand how they maintain social capital in an effort to predict future influencer-viewer relationship success.

Haridakis and Hanson (2009) suggested the shared connectivity between viewers on social network sites promoted feelings of affinity from one individual to entire communities of interest, such as the interactions between YouTube beauty influencers and their digital communities. New members to the community initially were drawn to it based on the desire to 
obtain new information, such as how to blend a foundation or for a unique Halloween make-up tutorial. Returning members sought out new information as well, as it was implied by these influencers that each video featured content different from the one before it. While the information may be similar, its 'newness' made it noteworthy to viewers.

Beyond demographic details, each influencer shared elements of her personality on the screen, such as dancing to favorite music or their interests outside of YouTube. This information allowed viewers to learn more about each influencer on a personal level, suggesting they could have shared interests beyond beauty tips, fostering deeper feelings of affinity toward the influencer. Once engaged with the community, the viewer could learn more about the influencer beyond her abilities with make-up, as each offered personal information about their lives. The communication appeared more directed toward an individual viewer, whereby the viewer feels connected to the influencer at a more emotional level, generating bonding social capital.

The analysis of three beauty influencers sponsored, affiliate/free product, and nonsponsored content suggested the need for redefining, restructuring, adding, and omitting variables from Sander and Lee's (2014a) model to account for a one-to-many social capital relationship. Modified definitions for five measures from Sander and Lee's (2014a) model are presented in Table 6 (p. 112), as relevant to YouTube beauty influencers. Modifications to the original model are defined within each construct as necessary. The addition of trust and reciprocity as forms of social capital are explored in more detail in the following section. 
Table 6.

Constructs of trust and reciprocity as a form of digital social capital, as adapted from Sander and Lee's (2014a) model

\begin{tabular}{|c|c|c|}
\hline Form of Social Capital & Construct & Definition \\
\hline \multirow[t]{3}{*}{ Trust } & Similarity & $\begin{array}{l}\text { Beauty influencers and viewers have actual and } \\
\text { perceived similarities in interests, skills, physical } \\
\text { characteristics, and personality traits which suggest } \\
\text { a relatability between network members }\end{array}$ \\
\hline & Credibility & $\begin{array}{l}\text { Opinions or insights of the influencer, removed } \\
\text { from any description or suggestion of advice, } \\
\text { which then may be assessed by the viewer }\end{array}$ \\
\hline & Impact & $\begin{array}{l}\text { Information provided by the influencer with direct } \\
\text { advice or measurable outcomes; viewers may } \\
\text { choose to follow or deny advice }\end{array}$ \\
\hline \multirow[t]{2}{*}{ Reciprocity } & $\begin{array}{l}\text { Involvement/ } \\
\text { Engagement }\end{array}$ & $\begin{array}{l}\text { Perception influencers create content that grows the } \\
\text { community and provides the opportunity for } \\
\text { additional interaction with the influencer and } \\
\text { community members, toward generating fair and } \\
\text { equal measures of reciprocity }\end{array}$ \\
\hline & Investment & $\begin{array}{l}\text { Actionable functions of reciprocity, including the } \\
\text { time and energy dedicated to the community by the } \\
\text { influencer, leading to rewards for both influencers } \\
\text { and viewers }\end{array}$ \\
\hline
\end{tabular}

This amended model proposes social capital was maintained by YouTube beauty influencers through a combination of trust and reciprocity as two forms of social capital. Mayer, Davis, and Schoorman (1995) defined trust as the "willingness of a party to be vulnerable to the actions of another party based on the expectation that the other will perform a particular action important to the trustor, irrespective of the ability to monitor or control that other party" (p. 712). The second form of social capital, reciprocity, was defined by Tayler (1982) as an exchange of short-term altruism for long-term self-interest and "cover[s] a range of arrangements, and relations and exchanges, including mutual aid, some forms of cooperation and some forms of sharing" (p. 28). Putnam $(1995,2000)$ proposed social capital was built on the tenants of trust 
and reciprocity, with Coleman (1988) further suggesting social capital was defined by its function. Similar to other types of capital, trust and reciprocity function together, creating social capital, which made it possible to gain certain resources otherwise unavailable (Coleman, 1988).

In regard to Sander and Lee's (2014a) suggestion that digital social capital was generated and maintained by access and motivation, the three influencers in this study surpassed these measures. As of December 2016, each influencer had amassed over 2,000,000 individual subscribers to her YouTube channel. Viewers easily were able to gain access to the community as the only requirements for membership was an internet connection and subscribing to the channel via their free YouTube account. When assessing motivation for these influencers to form deeper connections with viewers, the involvement and investment by these women may be partially influenced by the monetization of their content. Influencers were invested in creating content, as they must maintain their notoriety to retain viewers and increase opportunities for monetization. As each influencer in this study continued to gain subscribers and video views during the duration of this research, it was suggested that viewers were motivated to return to their channels for content based on perceived involvement and investment by the influencer. If access and motivation are no longer the mechanism for social capital maintenance once a notoriety threshold was exceeded, it then raised the sub-question: What practices are employed by YouTube beauty influencers to construct and maintain perceived social capital?

Trust as a form of social capital. At its core, trust is defined as a willingness to be vulnerable, to believe in another's competency, capabilities, reliability, or openness as a predictor of how a person will perform and behave (Mayer, Davis, \& Schoorman, 1995; Nahapiet \& Ghoshal, 1998). This study viewed trust as being one of two key forms of social capital, built from knowledge of uncertainty reduction (Coleman, 1998; Putnam, Leonardi, \& 
Nanetti, 1994). Trust is a form of social capital on which obligations and expectations of the individual could be based (Coleman, 1998).

Based on this conceptualization of trust as a form of social capital, Sander and Lee's (2014a) model of digital social capital provided three key measures from which trust could be defined: similarity, credibility/insight, and impact. In the original model, trust was included as a measure, not a direct form, of social capital. It was defined as the notion people need trust to exchange resources or information (Sander \& Lee, 2014a; see Figure 3, p. 74). As this research suggests trust as a type of social capital comprised of more specific constructs, this category herein will be referred to as credibility/insight. This differs from the construct of impact in that credible information was not backed by any description or advice. Rather, credible information was projected from opinions or insights of the influencer, which are assessed passively over time, through reputation and continued interaction.

As influencers gain followers, their similarities, credibility, and impact may be taken for granted as they have shown validation by the group. They have shown the ability to produce all three based on their status in the community. This then raised the question of how do influencers maintain their similarities, credibility, and impact once the validation has been achieved? One mechanism through which this was achieved was gaining the generalized trust of the community as a whole.

Similarity as a component of trust. Research findings suggested a strategy to reduce the risk associated with trust was to rely on individuals who share similar values and interests (Siegrist, Cvetkovich, \& Roth, 2000). Individuals with higher levels of trust often based knowledge on being similar enough to others to form social relationships, reduce risks associated with trust, and eventually generate social capital (Igarashi et al., 2008; Valenzuela et al., 2009). 
The connection between suggested similarity and trust was demonstrated by all three beauty influencers in this study, across each sponsorship category. In contrast to the other measures of trust as a form of social capital, all three beauty influencers projected similarity components of equal importance and type across sponsored, affiliate/free product, and non-sponsored videos. In regard to similarity, each influencer projected a notion of being relatable and possessing similar interests to the viewers. These statements regarding similarities to viewers fell into two predominant categories: (1) actual similarities and (2) perceived similarities (see Table 7 below). Table 7.

Matrix of actual and perceived similarity statements in video content across three YouTube beauty influencers

\begin{tabular}{|c|c|c|}
\hline & Actual Similarities & Perceived Similarities \\
\hline Caitlin & $\begin{array}{l}\text { "I am in the shade, just in case you } \\
\text { guys are wondering if we're the same } \\
\text { shade, I am in } 3 \mathrm{~W} 1 \text { Tawny." }\end{array}$ & $\begin{array}{l}\text { "I don't know about you guys, but the } \\
\text { glowier I get, the more glamorous I } \\
\text { feel." }\end{array}$ \\
\hline Danielle & $\begin{array}{l}\text { "It's [Dia de los Muertos] greatly } \\
\text { honored in many states in Mexico, } \\
\text { such Michoacán, which is where I was } \\
\text { born." }\end{array}$ & $\begin{array}{l}\text { "Going out with my husband has been } \\
\text { one of our priorities lately since our } \\
\text { anniversary which happened a few } \\
\text { weeks ago. And we really want to } \\
\text { spend more time with each other." }\end{array}$ \\
\hline Natalie & $\begin{array}{l}\text { "I think sometimes I just give myself } \\
\text { more breakouts because I'm stressed } \\
\text { out about my skin breaking out which } \\
\text { is making me more stressed and then } \\
\text { in turn just making me break out } \\
\text { more." }\end{array}$ & $\begin{array}{l}\text { "Today I'm going to do another make- } \\
\text { up tutorial. Because I just feel like } \\
\text { doing my make-up today." }\end{array}$ \\
\hline
\end{tabular}

Actual similarity. Caitlin, Danielle, and Natalie suggested viewers shared physical qualities with them, from ethnic backgrounds to similar shade ranges in foundation, which showed viewer-verifiable outward similarities. This actual similarity provided a type of baseline, from which assessments of values, interests, and behaviors were determined in further detail through repeated interactions. Thus, actual similarity served as an initial catalyst for attraction, providing the push for viewer engagement with content from the influencers, as people prefer to 
compare themselves to those who are actually like them (Byrne, 1971; Crocker \& Major, 1989; Montoya et al., 2008). This was essential in the beauty community as individuals initially sought out content as a means of gaining new information and techniques regarding make-up application and products. If a viewer was physically similar to influencers in some way, they were provided with a visual context from which to assess make-up products or techniques. For instance, if a viewer had a round face and deep set eyes, locating an influencer with similar physical qualities provided a type of mirror-image to her own facial characteristics. Instead of trying to imagine how a make-up tutorial would look on her face, this viewer was provided with an actual physical representation of how to apply the information presented based on her needs. Caitlin demonstrated a portrayal of this actual similarity when she told viewers, "I am in the shade, just in case you guys are wondering if we're the same shade, I am in $3 \mathrm{~W} 1$ Tawny." This statement provided viewers who shared a similar in skin tone with Caitlin additional information through this association. They no longer needed to research shade ranges in this foundation, as the viewer's physical similarity to Caitlin provided the information.

Influencers also relied on actual similarities to express deeper connections to viewers, such as similarities in race or ethnicity. In a Halloween tutorial, Danielle told viewers "[Dia de los Muertos is] greatly honored in many states in Mexico, such Michoacán, which is where I was born.” Danielle frequently referenced her heritage in video content, providing tips for Latina women specifically. In a foundation review, she told viewers, "And if you have sun spots like me, which Latina women actually are more prone to getting sun spots. I don't know if you guys knew that but it is a fact," then they could build up a second layer of foundation coverage with the product being reviewed, to conceal those spots. This information provided a high level of similarity to viewers with analogous ethnic backgrounds. If a viewer was born in the same state 
in Mexico as Danielle, there was further actual similarity to her, but at a less superficial level. An individual's race and ethnic background is more difficult to modify than hair color or foundation shade, providing a potential deeper level of connection for viewers.

This example also illustrates the issue of cultural appropriation in the beauty industry. Cultural appropriation is defined as “the use of a culture's symbols, artifacts, genres, rituals, or technologies by members of another culture" (Rogers, 2006, p. 474). Cultural appropriation is deemed problematic when it becomes exploitative, such as when elements of one culture are adopted by a more dominant culture without appropriate reciprocity, permission, or compensation (Coombe, 1993; Rogers, 2006). When individuals engage in acts of adopting culture and interpreting them as their own, without demonstrating a reciprocity or permission to engage in the act, it may be seen as cultural appropriation.

This was demonstrated in Danielle's video, where one viewer commented on Danielle's sugar skull Halloween tutorial stating, "Standing by your roots. I love it!! Represent for the Latinas!!” Another viewer noted, "Non Indigenous people - DO NOT USE SUGAR SKULLS AS A HALLOWEEN COSTUME." When non-indigenous influencers engaged in creating sugar skulls as a type of 'costume,' it was seen as exploiting the culture from which they carry significant meaning. The act was no longer seen as an extension of the cultural authenticity and heritage from which it carried significant symbolism, but rather was a costume, generated for pure entertainment purposes, creating an act of cultural appropriation. Danielle's discussion of Dia de los Muertos (Day of the Dead) and her actual connection to the ethnic heritage surrounding the holiday qualified her among viewers as being representative of her culture. Thus, Danielle's tutorial was received as a celebration of her heritage, authentic to the culture in which she identifies based on her actual similarity to viewers in ethnic background, rather than 
an act of exploiting cultures for entertainment purposes. When communication was directed at a specific group, the information appeared more personal and homogenized, which ultimately led to great feelings of affinity and the potential for generating emotional resources necessary to produce bonding social capital, through the actual similarity (Burke et al., 2011; Valenzuela et al., 2009).

Perceived similarity. Montoya, Horton, and Kirchner (2008) suggested as relationships grow and develop, perceived similarity produces stronger bonds between individuals. Initially, viewers were attracted to influencers based on their actual similarities to reduce the investment in finding solutions to their problems. As time progresses, the actual similarities reached a point of saturation for viewers, where being physically similar to an influencer was no longer enough to justify forming deeper bonds with them. A viewer could only view so many "glam" tutorials before they understand how to achieve the look on their own face.

To develop deeper relationships, Montoya et al. (2008) suggested individuals may begin to modify their attitudes or beliefs to match those they admire, trying to appear similar from a personality and moral stance. This related directly to bonding social capital. As communities begin to homogenize themselves, group membership based on actual similarities alone is not enough to dictate forming deeper bonds with others. In these homogenous groups, individuals are less likely to rely on information gain to maintain social capital. Instead, stronger ties are fostered by focusing on emotional resources, such as the exchange of similar values and beliefs. Instead of relying solely on actual physical similarities as a measure of likeness, viewers were willing to skew their own personalities to be closer to those of the influencer, to appear more similar to them beyond physical characteristics, suggesting the possibility for stronger ties to be formed. 
A primary example of how influencers utilized their personality traits to create perceived similarities with viewers was found on Caitlin's channel. Caitlin focused on humor, such as showing herself lip-syncing to music or taking "a Cheetos break" to eat a snack during one tutorial. She often relied on joking to interject personality into more instructive parts of her videos. In alignment with research on perceived similarities and self-images (Kressman et al., 2006; Nahemow \& Lawton, 1975; Stroebe, Insko, Thompson, \& Layton, 1971), viewers also began to project their own humor in the comments section, seemingly aligning themselves with an element of Caitlin's personality. One viewer commented on a glamorous make-up tutorial with her humorous take on how she would fair with the look when she stated, "tbh if I tried this I'd look like I got in a fight bc they ran out of guac at chipotle." The comment received 66 likes from commenters, with one fellow viewer stating "same omg lmaooo." Caitlin herself responded with smiley face emoji's and "OMG." There was an understanding among viewers' that humor was acceptable and encouraged on Caitlin's channel, as these comments were perceived as being similar to statements Caitlin herself made. Over time, the idea of being physically similar to an influencer was deemed less important, as it served as an initial indicator that an individual with visible similarities also may share emotional and behavioral similarities (Nahemow \& Lawton, 1975; Stroebe et al., 1971).

Social capital, trust, and similarity. Actual and perceived similarity illustrated a connection to elements of bridging and bonding social capital, through the projections by influencers and assessment by viewers. In regard to actual similarity, this made viewers feel more attracted to an influencer, as researchers suggested individuals prefer to compare themselves to those who are actually similar to them (Crocker \& Major, 1989; Montoya, Horton, \& Kirchner, 2008). One explanation for this may be due to bridging social capital. For instance, 
if a viewer was looking for a new eyeshadow to enhance her blue eyes, she likely would seek out a product recommendation from an influencer with blue eyes, as she had direct knowledge of the information desired. As bridging capital focused on information gain, viewers found it easier to gain access to information relevant to their needs from influencers who shared their physical similarities.

In regard to bonding social capital and similarity, Montoya et al. (2008) suggested as relationships grew and emotional resources were exchanged, individuals were more willing to modify their actions, attitudes, and beliefs to match those they aspired to be. This notion lends itself to construction and maintenance of a self-image, as an individual could perceive others values, beliefs, and personality factors to define their own self (Kressman et al., 2006; Sirgy, 1982). If viewers believed their self-image identified with the influencer, they may use other perceived similarities to reduce the dissonance between the actual and ideal self. As dissonance reduced, individuals could communicate with greater confidence and less effort, as seen in more intimate relationships (Selfhout, Denissen, Branje, \& Meeus, 2009). Thus, viewers who wished to engage with influencers on a more personal level - toward generating bonding capital - could rely more heavily on perceived similarities as a means of matching self-images.

Credibility as a component of trust. As all three beauty influencers in this study received monetary gains for their video content, the 'selling' of their content could be viewed as a reflection of the trust generated with viewers through their standing as credible sources of information, both at the bridging and bonding social capital level. Credibility is a direct type of trust, one rooted in "the belief that the other party is honest, reliable, and competent" (Ba \& Pavlou, 2002, p. 246). Findings further suggested individuals develop trust through credibility when they thought another party can perform a task effectively (Ba \& Pavlou, 2002; Doney \& 
Cannon, 1997). An essential element of credibility is whether an individual possesses the true expertise and resources necessary to match their promises (Cullen, Johnson, \& Sakano, 2000). However, this is a subjective knowledge, as there is no requirement to demonstrate these skills (Blomqvist, 1997). Instead, credibility is earned by conveying truthful information, further enhanced by the viewers' faith and confidence in their abilities (Kouzes \& Posner, 2011). Credibility, therefore, is a passive element of trust, as it is the perceived ability of another to perform something he or she claims to be able to do (Blomqvist, 1997).

In regard to beauty influencers on YouTube, the credibility they have gained with viewers reflected their standing in the digital community. Caitlin posted an average of 54.67 videos a year, or 1.05 videos a week overall, which she began on a weekly basis three years ago. Both Danielle and Natalie have maintained consistent uploading since beginning their channels. Danielle posted 900 videos in her past eight years on YouTube. She averaged 1.44 videos a week during this study, or 75 videos a year, as she streamlined her posts to a weekly basis. Natalie posted 511 videos in seven years, maintaining a fairly consistent average of 73 videos per year. All three of these influencers had a lengthy history of producing YouTube content on a consistent basis, with consistency serving as one point of credibility (Ba \& Pavlou, 2002; Blomqvist, 1997; Cullen et al., 2000; Kouzes \& Posner, 2011).

Additionally, these influencers gained a reputation in the beauty community, retaining millions of subscribers based on the content being projected. Herbig and Milewicz (1993) proposed to gain credibility, a reputation for quality must have been developed through fulfilling promises and generating positive feedback. Once a reputation has been formed - typically through producing consistent, dependable content over a period of time - viewers then may classify influencers as being credible sources of information. In the context of this study, 
credibility was observed through the opinions or insights of the influencer, removed from any actual description or suggestions of advice.

Credibility of the three beauty influencers in this study was assessed across nonsponsored, affiliate/free product, and sponsored video content (see Table 8 below). The credibility projected by the influencers in each of these videos produced varying levels of trust, and ultimately social capital, based on the content of videos and influencer relationship to the products. Viewers remarked on levels of credibility in different ways based on the presence or absence of sponsored, affiliate/free product, or non-sponsored content, in addition to the mechanisms by which influencers projected their credibility standings. 
Table 8 .

Matrix of credibility statements in non-sponsored, affiliate/free product, and sponsored video content across three YouTube beauty influencers

\begin{tabular}{|c|c|c|c|}
\hline & Non-Sponsored & Affiliate/Free Product & Sponsored \\
\hline Caitlin & $\begin{array}{l}\text { "... you got to try the Girlactik luster } \\
\text { face glow. It will change your life." }\end{array}$ & $\begin{array}{l}\text { "I feel like [the] Iconic [false lash } \\
\text { style] is pretty standard when you } \\
\text { want to go for glam. Because they } \\
\text { are the most perfect lashes for glam } \\
\text { looks." }\end{array}$ & $\begin{array}{l}\text { "So here I'm using the Clinique } \\
\text { (sponsor of video) Take the Day Off } \\
\text { balm, which is absolutely amazing... } \\
\text { Literally just dissolves all of your } \\
\text { make-up off of your face." }\end{array}$ \\
\hline
\end{tabular}

Danielle "The eye makeup is more spring and summer (she noted this was a "holiday glam" tutorial in the introduction), but I think paired with the lip, it makes it appropriate for fall and winter and the holiday season."

Natalie "I feel like maybe this [light pink lipstick] would be like a great dupe for one of [Kylie Jenner's lip kits]. I don't have any of [the lip kits] because ain't nobody got time to sit on the Internet and wait on those things."
"Alright so I want to take a stab at actually using the [eyeliners] as markers, since I did get a book for it. I want to show you because it might be more visible to see how much of a change can occur just by the amount of pressure that you can apply to the tip of the brush. So I am going to use all of the markers and I am going to try and paint something."

"I usually like to get them [the false lashes] in the four-pack. I don't know why, I just think lashes in the four-pack look better. That could just be me like being a weirdo..."
"I finish off every single look that I do with one of my favorite scents. This one is Viva La Juicy (sponsor of video) and it just makes me feel so feminine and flirty. It's playful glamorous all at the same time. And if I could describe it to you guys, it smells like delicious wild berries, with creamy vanilla, and jasmine."

"Moving on to the eyes, I'm using probably my favorite day shadow of life... (not a sponsored product for this video)" 
Credibility of the influencer and non-sponsored content. One mechanism by which beauty influencers displayed their established credibility with viewers in non-sponsored content was through suggesting favorite items, without needing to prove why it was a favorite. In a favorite products video, Natalie suggested a lip product she enjoyed "would be like a great dupe for one of [Kylie Jenner's lip kits].” The Kylie Jenner lip kits are a highly sought after product in the beauty community, notorious for selling out within minutes of their release (Schaefer, 2016). While Natalie suggested this product as similar to the popular Kylie lip kits, she followed up stating, "I don't have any of [the lip kits] because ain't nobody got time to sit on the Internet and wait on those things." This second statement shifted the focus away from being actionable information and placed it squarely as a form of credibility. While Natalie may have suggested the lipstick she owned was similar to the Kylie lip kit, she did not own any of the Kylie products, implying she was not able to actually test her suggestion. Instead, viewers must trust in her credibility with the color and formulation of the product to decide whether purchasing the suggested lip product will meet their expectations.

Apart from product recommendations, the beauty influencers also displayed their credibility with the viewers by providing additional descriptors in their video content. In a December 2016 non-sponsored video, Danielle told fans she was going to challenge herself to create a "holiday glam" look using products found at a dollar store. At the end of the video she stated, "The eye makeup is more spring and summer but I think paired with the lip, it makes it appropriate for fall and winter and the holiday season." There was no further discussion as to why the lip color selected reminded her of fall, winter, or the holiday season. With Danielle herself admitting the eyeshadows appeared to be more appropriate for spring or summer, her credibility was left to be assessed by the viewer, as she claimed to be able to achieve a glam 
make-up look from the dollar store, not a subtle spring make-up application. Based on this statement, viewers had to assess Danielle's overall credibility as an influencer to trust this tutorial was indeed appropriate for a "holiday glam" look. In the comment section, viewers commended her make-up look, as one viewer proclaimed, "I would NEVER guess that these were dollar store products! It looks amazing! Great job [Danielle]!" while another stated "The dollar store makeup looks perfectly glamorous on you..." Based on viewer feedback to this video, it appeared this contradiction between the intention of the video and the final look did not lower Danielle's credibility with viewers. Viewers noted her expertise with make-up overall, not with a specific product, complimenting her ability to achieve such a glamorous look on a budget, as she had promised. As such, credibility of the influencer in non-sponsored content was reinforced by viewers by accepting the general expertise of the influencer, rather than their product-specific abilities.

Credibility of the influencer and affiliate/free product content. In comparison to the non-sponsored video content, the affiliate/free product videos focused credibility on productspecific information gain. This content was focused heavily on product reviews or make-up tutorials, with the credibility of influencers in terms of product expertise being front-and-center.

Caitlin demonstrated this product expertise when she suggested to viewers that purchasing a popular product's competition would provide them superior results in their make-up application. In a December 2016 video, while applying foundation and concealer, she told viewers "[This sponge is] softer than the actual [well-known sponge product] and it works a little bit better in my opinion, which is a big thing to say because a [well-known sponge product] is like the best thing." There was no additional description of why the new sponge was superior to the popular alternative, outside of being softer. She even noted the alternative was "the best 
thing," suggesting it should have held some superiority to the new sponge. To help legitimize her opinion, Caitlin did demonstrate using the new sponge to apply product, however, she did not provide any additional product description or analysis of product capabilities. She projected to viewers it was her expertise with make-up application and familiarity with the competing products that made her opinion credible and worthy of acceptance.

Natalie demonstrated a suggested expertise with a product when she told viewers, "I usually like to get [the false lashes] in the four-pack. I don't know why. I just think lashes in the four-pack look better." While Natalie's initial statement could have caused some viewers to question her credibility, as this was truly an opinion based comment with Natalie herself referencing she didn't know why she liked the product better, one viewer provided agreement, stating, "I agree with you when you say lashes look better in the four pack! haha." This comment received 146 likes and four replies, of which three replies noted they too agreed four-pack lashes look better. In this instance, while the viewer provided the legitimization, it still functions to bestow credibility to Natalie as an expert and suggests her opinions carry a significant weight with viewers.

In the affiliate/free product video category, most viewers assessed influencer credibility based on their expertise with products specifically, not their overall abilities with make-up. This likely was due to the informational tone the influencers projected in these videos. Of the 15 videos affiliate/free product videos reviewed across all three influencers, 13 of the videos featured a make-up tutorial or product review element. The inherent expertise of the influencer was displayed heavily in these videos, with the credibility of the opinions being backed by the competence and reliability demonstrated by influencers over time (Blomqvist, 1997; Erdem \& Swait, 2004). 
Credibility of the influencer and sponsored content. In regard to sponsored content, influencers continued to rely upon their expertise to place sponsored products or brands as being superior to others. These videos also featured information-heavy content which contained larger amounts of physical demonstration of products provided to influencers from the sponsoring brands. While influencers did not directly state if information had been scripted by the brand, there was an obvious change in tone during these segments, making them appear almost commercial in form.

In regard to sponsored posts, there were a variety of ways in which influencers engaged in sponsorships. However, the only federal regulation for sponsored content was it had to be labeled with the proper FTC disclaimer to make it apparent to viewers' content was paid (Wu, 2016). Beyond this regulation, how influencers promoted products or services from sponsors varied drastically from video to video. Most sponsored videos featured a short clip where influencers promoted a product for one minute or less, such as a video by Natalie where she exclaimed, "[The sponsoring company is] a new lifestyle brand... I like them because they produce their products in really small quantities to preserve the quality of their products." These instances typically revolved around the influencer creating content related to the sponsored good but featured other products as well, situating the paid content as being just a short piece of the whole look.

Other sponsored posts appeared to require influencers to promote a single brand, featuring only their products or services in the paid content. This occurred in Danielle's channel during a hair care routine, in which she only mentioned products from one specific brand. Overall, influencers appeared to prefer the first option, in which they featured a sponsored 
product for a short period of time in secondary content, as this was the type found in $67 \%$ of sponsored videos across all three influencers.

As beauty influencers engaged in direct paid product placement in these videos, it came as little surprise they were more apt to note brand names directly or promote sponsored products as being their favorites. Danielle suggested she, "can't live without" a certain hair care brand as she had "been on the hunt for a product that keeps [her] waves alive without having to work at it." Caitlin professed to viewers she used a sponsored brands make-up remover as it was “absolutely amazing." Each influencer proclaimed products from the sponsor to be favorites of theirs and briefly explained why based on their experience. However, she then spent an average of one minute or less featuring the sponsored product, with the largest proportion of time spent discussing non-sponsored products.

As a result, viewers noted instances on all three influencers' channels when sponsored content appeared to be less-genuine, potentially damaging the influencers' overall credibility. Caitlin received the highest level of criticism for a single video, a make-up tutorial sponsored by a drugstore cosmetics brand in which viewers questioned her judgment in not verbalizing it was a sponsored post. Caitlin responded directly to viewers telling them, "After this video I have two more [drugstore brand] sponsored videos which I will be sure to put in the title so that you all are aware right away." Viewers seemed satisfied with her transparency based on this comment, as she was honest to the community and explained how her future sponsorships would be labeled. As it is suggested credibility was awarded to influencers based on their honesty and dedication to the community (Kouzes \& Posner, 2011), Caitlin could stabilize her credibility with viewers through providing this promise to them. 
Natalie also was questioned by viewers in a hair tutorial, when she chose to use bobby pins that were a different color from her hair so they stood out against the intricate braids she had created. Viewers questioned Natalie's credibility and expertise in creating the content, due to the obvious mistake, with viewers stating, "What kind of tutorial was that [Natalie]? Is she serious?" and another questioning why she did not take the extra time to obtain bobby pins the same color as her hair. This suggested a decrease in quality across sponsored content that did not meet the expectations set forth by influencers and could be perceived as a direct result of their sponsorships; it appeared that creating content was only important to their monetary gains, not for the community's information-gain.

Of further interest were the discrepancies between the two types of sponsored videos. Influencers showed a preference for promoting sponsored brands within content which was otherwise non-sponsored. Viewers also were less apt to question sponsorships and influencer credibility when the sponsorship was less apparent. This could suggest viewers see this type of sponsorship as being less impactful overall. The information presented was still relevant and deemed credible by viewers, as the entire tutorial or review was not dedicated to a single product. The products were carefully placed and justified by influencers, telling viewers why a sponsored product was their favorite and positioning it as being relevant to the information being presented. In contrast, videos focused on a brand as a whole were more commercialized, as if they were pushing the products rather than suggesting how they fit in with their current routine. Viewers noted this discrepancy as well, discussing how the quality of the information presented seemed lower in these fully sponsored posts. Influencers could have shown a preference for the option of a single product placement, as it was deemed more credible by viewers. By integrating the product into their typical routines, the information appeared more genuine. The content had a 
less commercialized tone and more informal approach, which was what viewers responded positively to in other aspects of social capital.

Social capital, trust, and credibility. This discourse suggested social capital generated through the trust of an influencers' credibility may be influenced by viewer intention. As Ellison et al. (2011) suggested, bridging social capital is of importance as it stems from the sharing of information across individuals with diverse backgrounds. By sharing information with viewers, even in the form of opinions and insights, the influencers provided a new context for the shared content. Viewers who watched these videos to gain bridging social capital may be more apt to judge credibility based on the apparent expertise of the influencer or purpose of the video. As demonstrated in the sponsored postings, viewers who sought out information toward gaining bridging social capital may deem influencers less credible when the content seems contrived, suggesting influencer opinions were not based on genuine feelings and knowledge of the product, but on monetary gains. The relative positivity in terms of influencer credibility in nonsponsored and affiliate/free product videos also supported this notion, as content in these categories was responded to by viewers as being more credible.

This research suggested the credibility established in non-sponsored and affiliate/free product content could not always be relied upon to translate to sponsored videos. As credibility was established over time and was based in consistency, there was an apparent deviation when influencers created sponsored content on their channels. All three influencers began their channels as an outlet to engage community members with similar interests in beauty and to demonstrate their expertise, therefore, there was a perception among viewers this should continue to translate into their current content. While there was an understanding influencers must generate revenue at some point to maintain their content, such as to purchase new products 
or equipment for editing, there was an underlying sense of distrust among viewers in sponsored content. The credibility of the influencer was impacted, as the intention of the video itself was questioned. As influencers are not required to show tangible proof of their perceived credibility, the act of engaging in sponsored content appeared to push viewers to question whether these statements are true reflections of their expertise or if they are the side notes of monetary gains.

Impact as a component of trust. When assessing ability, there must be a discussion of intent versus practice, considered in this study as credibility and impact. The notion of ability in producing trust suggests an individual may possess some skill or competency which enables him or her to have influence in a specific community (Mayer et al., 1995). Credibility is considered the passive aspect of ability, where individuals trust another to have the expertise to complete a task, building of a reputation over time without necessarily showing the ability (Ba \& Pavlou, 2002; Blomqvist, 1997; Doney \& Cannon, 1997). In contrast, impact is created by providing actionable advice, which then may be accepted or denied by another party (Swol \& Sniezek, 2005).

While providing actionable advice was demonstrated by all three influencers in this study, one important aspect of this construct was that advice may be accepted or denied by viewers. The denial of advice did not necessarily mean a viewer did not value the information. Tripathi, Caplan, and Naidu (1986) suggested the rejection of advice may be due to individuals generally being uninterested in solving a problem. As trust was rooted in uncertainty reduction, the time and effort required to assess credibility and follow impactful advice may be deemed unnecessary by viewers if there was not a genuine need for information to solve a problem (Mayer et al., 1995; Tripathi, Caplan, \& Naidu, 1986). Viewers also may deny following advice if it was deemed to be objectively bad (Tripathi et al., 1986). This did not suggest the advice 
itself was bad or meant to cause harm to viewers. Instead, a viewer may deny advice if they thought it would not work for their personal needs. For instance, if an influencer recommended a foundation to control oil, a viewer with dry skin may deny the advice as using the foundation could in fact dry out his or her skin. The information was not inherently bad advice, however, it may not be the most applicable to all viewers. Thus, viewers may deny the information presented by influencers, while still acknowledging the impact of the content being provided.

Although there is the opportunity to deny advice, many individuals actively seek out and accept advice from those with higher expertise and ability. McGrath, Vance, and Gray (2003) suggested individuals accept impactful advice as a means of filling in gaps in their knowledge, resolving problems, or taking advantage of opportunities more quickly. In returning to the previous example, if an influencer suggested a foundation to control oil, then viewers with oily skin may gain an advantage by accepting the influencer's advice to purchase the product. By following this advice, the viewer would be able to resolve a potential problem (oil control) and take advantage of the opportunity to do so more quickly than they may have with their own research. Therefore, following the advice of an influencer could prove advantageous to viewers whose needs match the information projected by influencers.

Impact of the three beauty influencers in this study was observed across non-sponsored, affiliate/free product, and sponsored video content (see Table 9 below). The advice projected by the influencers in each of these videos could be accepted or denied by viewers based on its relevance. While the influencers shared similar types of impact statements across the three categories, the context of the statements varied based on sponsorship status, similar to the credibility construct. The following section will discuss what type of information across all three 
sponsorship types was presented as impactful advice by influencers. Viewer responses also were included in this discussion. 
Table 9.

Matrix of impact statements in non-sponsored, affiliate/free product, and sponsored video content across three YouTube beauty influencers

\begin{tabular}{|c|c|c|c|}
\hline & Non-Sponsored & Affiliate/Free Product & Sponsored \\
\hline Caitlin & $\begin{array}{l}\text { "Now if your eyes are a little bit more } \\
\text { wider and spaced apart, you can really } \\
\text { go in whenever you're using those } \\
\text { dark shades right here because it's } \\
\text { going to balance your eyes out with } \\
\text { your face." }\end{array}$ & $\begin{array}{l}\text { "And sometimes it takes a little } \\
\text { while for it to build the coverage but } \\
\text { if you just let it dry and then apply } \\
\text { another layer. Let it dry, apply } \\
\text { another layer. Then you'll get a very } \\
\text { natural full coverage." }\end{array}$ & $\begin{array}{l}\text { "...if you have a Beauty Blender, } \\
\text { don't like smear it around...If you } \\
\text { want the most coverage and the most } \\
\text { bang out of your foundation, you } \\
\text { always want to press into your skin." }\end{array}$ \\
\hline
\end{tabular}

Danielle "Guys, if you travel a lot, take some makeup removing wipes with you and then when you get on the airplane, wipe it all off [the makeup] and then tone your face with this, moisturize... And then when you wake up, put on some tinted moisturizers, after you wash your hands, and your skin is not going to stress out from the, from, from just traveling."

Natalie "And then to make our highlight melt into our skin, I like to dampen my [sponge] with a little bit of [setting spray] and then just pounce that right over the highlight to get rid of any of the powdery look and just make it look like our skin is just glowing from within."
"This is a tiny little wand that I like to use to backcomb my eyebrows... This is going to give your hair some lift so it's not going to be flat and almost painted and then you just want to brush the wand lightly over it."

"And then I start at the front of my brow but a little behind it and outline that part. And then fill it in and then I will work my way to the front... So I just like to use short, fast strokes in the front of my brow to ensure that I'm not using too much product..."
"I'm going to start this look by using rattail comb to part my hair on the left side... You can also scoop the backcomb into a "C" shape or a scoop. That will really help you create the volume that you desire... Don't pull it too tight because you don't want to lose any volume."

"So the reason we lay down the black on the lid is, one, when you're using black eyeshadow, it can kind of sometimes transform and turn to more gray. So by putting down a black base you're just going to help yourself with creasing and you're going to help it stay darker." 
Non-sponsored content and impact statements. When assessing non-sponsored content, influencers presented impactful information based on actual similarities. These statements suggested products or techniques may work well for certain viewers, if they shared actual characteristics with the influencer. Caitlin suggested, "This foundation works really well for textured skin because it has like a primer-like fill to it. So, it really fills in all the fine lines and large pores." Danielle focused in on providing specific information to her viewers with oily skin, suggesting to them,

If you do have oily skin, make sure you use an oil control or mattifying primer on your T-zone and then add the luminous foundation on top. That way your t-zone, which is more prone to, you know, releasing oils, natural oils from the face, they're not going to build up immensely and you can still wear that beautiful dewy look, without being oily.

Both Caitlin and Danielle provided suggestions that their recommendations would match the need of viewers who possessed similar problems. Danielle proposed a solution to viewers with oily skin to achieve a luminous foundation look without generating more oil. Those viewers with oily skin may feel compelled to follow this advice, as it would help them solve a problem and generate a quick solution, while viewers with dry skin may choose not to follow this advice, as it would not solve a problem they have with their skin. This did not imply the information was bad for viewers with dry skin, just not directly applicable, based on their actual skin differences from Danielle.

Influencers also provided impactful statements based on personal expression, as related to abstractions of actual similarities. In a Halloween make-up tutorial, Natalie demonstrated visually how to apply face paints to create a skeleton look utilizing a voice-over to provide viewers with additional suggestions on how to mimic the look. She explained to viewers how to create a "splatter effect," to create a more abstract look to the face paint, stating, 
...I'm using just a stiff teasing brush. I'm going to dunk it in some water and use my water-activated face paints... And I'm just running my thumb over it to get the paint to splatter on me. Now you can change how your paint looks, how your splatter effect looks, just how fast you run your finger across it or how close or far away your holding the brush to your face.

This comment did not propose any actual similarity to viewers, but rather suggests they may take the advice to achieve a result similar to Natalie's. The viewers may follow this advice to create a similar look or deny the advice if they do not find the make-up look to be relevant to their expressive needs. Thus, while this comment did not assess viewers skin tone, eye color, or facial structure, it did offer actionable advice for those wishing to achieve a similar effect.

In comparison to sponsored or affiliate/free product videos, in non-sponsored content viewers were more likely to ask for advice directly from the influencers, actively seeking impactdriven statements. The impactful comments made in these videos provided knowledge to the viewers, allowing them to solve problems they may have or explore more creative expression (McGrath et al., 2003). Of the 15 videos in the non-sponsored category, viewers asking for direct advice from influencers accounted for $10.67 \%$ of the total comments, twice as often as in affiliate/free product or sponsored videos. Viewers on Caitlin's channel asked for specific product recommendations. One viewer asked, "Can you do a video on: Best \& Worst Stick Foundations | Drugstore \& High End?," while another asked specifically "WHATS ON YOUR NAILS THOU?” Viewers asked for similar recommendations on Danielle and Natalie's channels, asking "what are you wearing on your lips [Danielle]?" and "How do you get those beautiful waves? Can you do a hair tutorial on this look? :)," respectively. Viewers actively sought out advice in these videos, suggesting they enjoyed the information currently provided by influencers and requested additional content to suit their problems and needs directly. 
Affiliate/free-product, sponsored content, and impactful comments. In both affiliate/free

product and sponsored videos, influencers provided advice based on intention, focused on how to use specific products or demonstrating new techniques, without directly mentioning similarities to viewers. Caitlin showed viewers how to use a brush and explained a technique to blend out eyeshadow efficiently,

Whenever you apply your eyeshadow, you always want to start off with using a lighter hand. Put your hand [at] the end of your eyeshadow brush [demonstrates this]. Don't hold it too close right here [moves hand down the brush and mimics blending eyeshadow this way] because then you have too much control of the brush. You're gonna want to push down harder to put too much product down. You're not going to blend it as easy.

Caitlin's advice did not imply relevance to a specific type of viewer, such as one with close set eyes or a narrow nose bridge. This advice was adaptable, allowing it to be adopted by viewers across a variety of diverse characteristics, instead of just those who share a physical similarity with Caitlin. Natalie also employed this broad-based approach to advice, stating in a sponsored video post,

...the reason we lay down the black on the lid is, one, when you're using black eyeshadow, it can kind of sometimes transform and turn to more gray. So by putting down a black base you're just going to help yourself with creasing and you're going to help it stay darker.

Similar to the information provided by Caitlin, this content suggested viewers may benefit from knowing this information if they are interested in creating a dark eye look. There was no qualification to utilize this advice on a personal level, other than possessing the products necessary to achieve the look. Viewers did not have to have a similar eye color or shape to influencers, as this advice was focused on improving technique, and how it could be adapted across a wide-range of viewers. 
Beyond providing information about technique, sponsored videos required influencers to engage in impactful conversations about specific products to meet the requirements of the paid sponsorship. Caitlin told viewers a sponsored lipstick was a good product because,

...it's really comfortable when you're putting it on your lips. This is a unique lippie because it glides on like a gloss but it gives you that intense rich color of a lipstick. It's also very nourishing to the lips and its extremely comfortable. It's very, very wearable for every day. It doesn't feel like you're wearing something thick and heavy on your lips. It just sits nice and comfortable. You won't even realize you're wearing it throughout the day. This is really the first of its kind!

Differing from credibility, these impact statements were actionable. Caitlin told viewers why this lipstick was unique, providing qualities which could be physically assessed by viewers, such as moisture level and intensity of color. Influencers implied this product-specific advice provided viewers the solution to a potential problem, such as dry lips or finding a bold red shade. These impact statements promoted opportunity gains through problem solving, as the product mentions typically were focused on it producing superior results. Thus, accepting this information could reduce the time and effort necessary in testing products and formulating opinions, as the advice originated from the trusted expertise of the influencer (Briggs, Burford, De Angeli, \& Lynch, 2002; Constant, Sproull, \& Kiesler, 1996; Swol \& Sniezek, 2005).

Social capital, trust, and impact. In regard to impact statements, a viewer's intent ultimately could impact the nature of the social capital derived, as viewers utilized this actionable information for different reasons. In regard to impact, bridging capital may be a way to gather advice toward opportunity and innovation, as it provided novel information across diverse backgrounds (Constant et al., 1996; McGrath et al., 2003). In contrast, bonding capital was shared between strong ties, where advice was focused on problem solving or reducing uncertainty (McGrath et al., 2003). In non-sponsored content, influencers were more likely to share advice which was personally driven, suggesting an actual similarity between the parties. 
Non-sponsored content also focused heavily on providing information to viewers in the form of technique, rather than direct product suggestions. These statements were focused toward helping viewers solve a problem they may have, such as creasing eyeshadow or dry skin patches, rather than suggesting novel information about specific products. As viewers sought out and judged advice based on their needs - from opportunistic information gathering to problem solving - the overall impact of an influencer's comments may have been accepted or denied based on the social capital desired.

In regard to statements of impact, the differences between non-sponsored, affiliate/free product, and sponsored content hinged on the format of the statement rather than direct questions of the content by viewers. Viewers did not have to assess whether these statements were true or not, as they were actionable. The influencer had shown the viewer how a product performs or a new technique, rather than implying these notions based on their credibility. The impact construct of trust appeared to function as a type of anchor statement. Influencers utilized impact to ground their content in facts and demonstrations, suggesting the resources they were providing had a function and purpose. There was little room for interpretation by viewers in impact comments; they could have chosen either to accept or deny the advice, however, this did not change the intention from which it was provided (Briggs et al., 2002; Constant et al., 1996; Swol \& Sniezek, 2005; Tripathi et al., 1986). These impact statements served as a point of reference for influencers and viewers, allowing both parties to see the benefits of advice giving and receiving, providing an actionable form of trust to the resources being provided.

Reciprocity as a form of social capital. Putnam's (2000) seminal piece, Bowling Alone: The Collapse and Revival of American Community, proposed social capital was built on the tenants of trust (previously discussed) and norms of reciprocity. Tayler (1982) defined 
reciprocity as a series of acts in which altruistic tendencies typically benefit every party at some point in the interaction, suggesting,

Each individual act in a system of reciprocity is usually characterized by a combination of what one might call short-term altruism and long-term selfinterest: I help you out now in the (possibly vague, uncertain, and uncalculating) expectation that you will help me out in the future (p. 28-29).

While short-term reciprocity may seem based on altruistic qualities at first, most relationships see the benefit of reciprocity in the form of a return on their investment. For instance, taking the extra time to rake leaves out of your neighbor's yard may seem purely altruistic at first, however, the behavior is reciprocated when your neighbor mows your lawn the following week. Based on Putnam (2000) and Tayler's (1982) definition of reciprocity, these actions were not based purely out of kindness and goodwill, but rather on the notion any costs obtained by completing one act would be repaid by the benefits of receiving a reciprocal act in return.

In reframing Sander and Lee's (2014a) model to be directly applicable to YouTube beauty influencers, refined definitions for involvement/engagement and investment, the two proposed measures of reciprocity, were necessary. In regard to these influencers, involvement/engagement was seen as the reciprocal acts of influencers and viewers toward increasing the opportunities to collect information, grow the community, and enhance relationships, based on network size. As Sander and Lee (2014a, 2014b) proposed, social networks can be large and expansive, with members who have different contacts and history to the network. Therefore, to grow the community and gain access to additional resources, influencers had to be willing to engage with viewers and provide resources deemed of fair and equal value to viewer involvement. The second construct, investment, demonstrated a time function of reciprocity (Portes, 1998). Influencers and viewers had to be willing to invest their 
time and energy into the community to gain additional resources. There needed to be a noticeable investment by influencers, in the form of creating frequent content, offering unique advice, and offering emotional resources for viewers to feel the reciprocity they devoted to the influencer's channel was meaningful. Through involvement/engagement and investment practices, influencers and viewers can see valuable results in reciprocity and increase the overall capital in the social network.

Involvement/engagement as a component of reciprocity. One means by which influencers could meet the demand of reciprocity was through engaging with viewers, demonstrating influencer resources are of great value. As Chiu, Hsu, \& Wang (2006) proposed, the involvement of influencers in a digital community provided reciprocity to viewers deemed fair and even in value to their commitment. It was through engagement with others that individuals were able to broaden their own knowledge, based on sharing their own resources and gaining new ones through the reciprocal actions of others (Billett, 1998).

In regard to YouTube beauty influencers, this suggested influencers must invest beyond simple information gain, showing their viewers they were dedicated to growing the community and providing additional opportunities for engagement, in return for reciprocal actions by viewers. This engagement was valuable to the community at large, as the size and impact of the network ultimately could affect the coordination and exchange of resources (Sander \& Lee, 2014b). This engagement, however, often required involvement beyond creating content. Viewers must feel as though they are engaged in a fair, reciprocal relationship, where input equals output (Chiu et al., 2006). Examples of statements from this research projecting influencer involvement/engagement can be viewed in Table 10 below. 
Table 10.

Involvement/engagement statements in video content across three YouTube beauty influencers

Caitlin "I want to know what type of skin types I have watching... I want to know like what do you look for in a foundation. Do you look for sheer coverage? Or you just want your skin to shine through? Just add a little bit of a healthy glow to it. What kind of skin type do you have?"

Danielle "If you guys want to do the tag, that would be really awesome. Or if you just want to tag me on Instagram, I would love to see what you come up with using dollar store makeup."

Natalie "Leave me some of your favorites in the bottom bar so I can check out some new things. Cause I'm always down to check out what you guys are into. And leave me some video requests down below of anything you guys want to see."

Involvement/engagement: Projections by influencers. All three influencers utilized the involvement/engagement category to request viewer feedback and suggested they wanted to create content relevant to their audience. In an affiliate video discussing her favorite products, Natalie requested viewers, "Leave me some of your favorites in the bottom bar so I can check out some new things. Cause I'm always down to check out what you guys are into." Danielle further explained that viewers had to tell her when they would like to see certain content, so she could produce engaging and helpful videos, stating "And if you guys see anything that you really like or a specific swatch video, you guys have to request it or else I don't know if you really want to see it." These statements suggested influencers wished to provide content to viewers that allowed them access to new information and forms of capital. By asking viewers to provide their feedback and suggestions, it implied influencers were willing to create content relevant to viewer needs and appreciated viewer opinions as another way to broaden the reach of the network.

Influencers also generated high levels of engagement by suggesting viewers extend their involvement beyond YouTube. Influencers mentioned viewer feedback from other social media platforms or invited viewers to connect with them on other sites. In a non-sponsored make-up 
tutorial, Caitlin thanked her "tweet fam" on Twitter for helping her to choose a new nail color, stating 'I'm very excited about my blue nails by the way. Thank you tweet fam... I asked you guys what colors I should choose and you guys chose this one. I love it! I love it a lot!’ By acknowledging her followers on Twitter, she could thank them for their involvement, reciprocating their action through generating an emotional resource by suggesting they have impacted her life in a positive way.

Danielle also acknowledged specific viewers but chose a different type of comment. Instead of focusing on funny statements or factual information from viewers, Danielle addressed a much deeper issue one viewer had with her video introduction. She typically opened every video with the phrase, "Hey, guys!" One viewer commented she "felt offended and she said that she felt [Danielle] was belittling women," by referencing "guys" specifically in her opening. Danielle used the opportunity to discuss the comment with viewers, explaining she tends to call everyone "guys" regardless of their gender, and used the phrase in her videos as she considered her viewers as friends and thought they should be treated with the same regard. This phrase was actually an act of bonding social capital, as she was close to her viewers, considering them her friends, and used the same speech patterns in her video content as she did in her personal life.

While this comment was directed at Danielle directly, she utilized the statement to further engage viewers, telling them her opinions and then stating, “...I want to know how you guys feel about it." Viewers responded to her prompt, utilizing the comments section to provide their opinion on the original post. Most agreed her use of the phrase "hey guys" was inclusive. Some offered constructive feedback, suggesting Danielle changed her opening to say "Hey, everyone" or to language inclusive of all genders. As a whole, the comments were positive and focused either on agreeing with Danielle's opinions or offering her constructive ways to create a more 
inclusive channel environment. This opportunity for engagement generally was seen as very positive by viewers, suggesting they appreciated the opportunity to provide feedback and involve themselves in a substantial aspect of Danielle's channel.

In contrast, for sponsored content viewers commented on the amount of paid posts in a certain time period and suggested the content seemed less engaging, as it had to be structured in a certain way to meet sponsorship criteria. One viewer noted,

i love [Danielle] and her opinions and lifestyle and I have been watching her since the very beginning but nowadays.. i dont know if it's just me, but I feel like she does videos only with partnerships. I mean, if its a tutorial, I know in advance that it is a partnership. I get the philosophy of [YouTube] as a job and sponsorship and they have a right to do what they want [and] make money as the want. it's just that [YouTube] nowadays is all about sponsors, but it would be nice if you just from time to time make a video just with your own products and by your own decision...

This comment suggested influencer content may be seen as less engaging and impactful when they were adhering to the protocols typically required in sponsored content. Viewers wanted to connect with influencers, to share information and emotional resources, not feel as though they were recipients of a product pitch. There is knowledge YouTube beauty influencers must monetize their videos to support their channels growth, however, viewers proposed this should carefully be integrated with more involving content, such as life updates or nonsponsored tutorials, to show they are still dedicated to growing their network and providing personal resources to viewers.

This research suggested involvement on YouTube may be seen as an expectation, rather than a benefit, of being in a digital community. Regardless of sponsorship type, viewers wanted influencers to listen to them and produce content that would help to grow the community at large, providing new opportunities for resource exchange. This relates to Kaplan and Haenlein's (2010) suggestion that those engaged in social media for monetary gains must be active and 
interesting. In regard to involvement, YouTube was less about explaining why your content was helpful and informative, and more about engaging others in an open conversation. Viewers wanted to feel as though they were part of the community and resource exchange was equal opportunity, based on the size and strength of the network. Similarly, influencers had to be willing to listen to viewers and create content they found enjoyable, content they would like to both hear and talk about. If influencers did not listen to their community, they risked members leaving to find others who were more involved with their needs. Engaging with the community appeared to be expected on YouTube, where involvement was linked directly to growing bonds and building the relationships necessary to engage in reciprocal actions between influencers and viewers.

Social capital, reciprocity, and involvement/engagement. Viewers first demonstrated a simple form of involvement, whereby dependable, but diverse, information from influencers fulfilled their needs and caused them to engage in reciprocal actions (Tayler, 1982; Putnam 2000). This reciprocity was linked directly to knowledge sharing, through bridging social capital, where the quantity and quality of information was assessed by viewers as the basis for fair and even measures of reciprocity (Chiu et al., 2006). These viewers were focused on information gain, motivated by gaining accesses to new knowledge resources, rather than emotional bonds (Burke et al., 2011; Valenzuela et al., 2009). If viewers were provided the content they desired, through the growth of the network, access to new knowledge resources, and quantity of information, they were more likely to engage in reciprocal actions, as their needs were being met fairly. In regard to bridging capital, this research suggested the higher the perceived influencer involvement and the larger the quantity of information gained, the greater the positive reciprocity demonstrated by viewers. 
As these networks grew through the involvement of the influencer and viewers, pure information gain became a secondary factor of reciprocity, due to the emergence of bonding social capital. As members remained in the community, they tended to homogenize, focusing less on gaining new information and more on involvement as a personal act (Valenzuela et al., 2009). The sharing of emotional resources, such as encouraging messages or acts of support, moved the social capital from bridging to bonding, producing stronger relationships and greater perceived involvement through emotional connections. Once viewers began to rely on bonding capital for their involvement, they see information gain as being a benefit, but not the sole source of their engagement with the influencer. Instead, viewers who utilized bonding capital for engagement tended to focus on the quality of the content produced, and whether it provided viewers with emotional resources, as a marker for fair-and-equal reciprocity.

Investment as a component of reciprocity. While engagement focused on the direct act of growing the network to provide fair and equal levels of involvement by all parties, investment was rooted in actionable time and energy. One researcher suggested the reciprocity generated through investment was proportional to what originally was invested in the relationship (Silverstein, Conroy, Wang, Giarrusso, \& Bengtson, 2002). Additionally, returns on investment grow with time, as individuals may wish to assess the level of investment prior to showing all the resources they can offer (Roberts \& Sherratt, 1998). These two notions suggested reciprocity rooted in investment also was a function of time, where individuals must have a baseline of actions to judge further investments. If the original investments were deemed equal in reciprocity to others, then additional acts of investment may continue to lead to greater rewards for both parties (Roberts \& Sherratt, 1998). 
With investment, there was a period of 'testing the waters,' as time and energy are both valuable resources. If an influencer invested a large portion of her time and energy into creating content that only garnered ten new subscribers over time, the reciprocal act by viewers may not be seen as equal and could cause the influencer to spend less time and energy creating content. Conversely, if influencers saw a great return on their investment in the form of increased views, likes, subscriber counts, and sponsorship opportunities, spending more time and energy creating content produced even greater rewards for them. Thus, investment was based on action, where influencers and viewers could assess reciprocity based on the perceived time and energy demonstrated by both parties, rather than more abstract perceptions of involvement.

Investment also was unique in it was the only measure of social capital in this study to include tangible assets, where influencers could provide actual resources to viewers in exchange for reciprocal actions. The overall investment by influencers was assessed by time and energy projections. The response by viewers served as a measure of determining whether the investment was fair and equal in reciprocity. All three influencers demonstrated their investment in similar forms, across the three sponsorship categories (see Table 11 below).

Table 11.

Investment statements in video content across three YouTube beauty influencers

Caitlin "Also, I have a vlog now with my fam... And that vlog is going to be over there. So, if you haven't checked it out, you might want to check it out."

Danielle "Thank you guys so much for all of the support from last week or from this week actually. I have been so excited that I have been on a roll. Third day in a row that I am filming a new video for you guys. And tomorrow I have another one."

Natalie "So if I'm gone for a little two week period of time, just know that I'm vlogging in that process and I will be back [after moving to a new house]..." 
Viewer projections of investment. This discussion of investment by influencers first will begin with assessing uploading statistics, as they suggested the time invested by influencers to their channels. A comparison of viewer uploading statistics can be viewed in Table 12 .

Table 12.

Uploading frequency, percentage of uploads in each category and uploads per week for each influencer from January 2016 - December 2016

\begin{tabular}{llccc}
\hline \multirow{2}{*}{ Caitlin } & Sponsored & Videos Posted & \% of Uploads & Uploads per Week \\
\cline { 3 - 5 } & Affiliate/Free Product & 15 & $26.32 \%$ & 0.29 \\
& Non-Sponsored & 27 & $26.32 \%$ & 0.29 \\
Danielle & Sponsored & 13 & $47.37 \%$ & 0.52 \\
& Affiliate/Free Product & 17 & $24.53 \%$ & 0.25 \\
& Non-Sponsored & 23 & $32.08 \%$ & 0.33 \\
\multirow{3}{*}{ Natalie } & Sponsored & 12 & $43.39 \%$ & 0.44 \\
& Affiliate/Free Product & 16 & $28.57 \%$ & 0.23 \\
& Non-Sponsored & 14 & $38.10 \%$ & 0.31 \\
& & $33.33 \%$ & 0.27 \\
\hline
\end{tabular}

The influencers uploaded an average of 0.98 beauty videos per week in 2016 . As this study aimed to address beauty specific content from these influencers, only videos related directly to beauty were included in these measures. Based on their uploading frequencies, certain assumptions of time dedication can be assessed for each influencer. Yet, in addition, investment in the content must be analyzed from a qualitative perspective to determine how influencers defined their personal investment to the community.

Influencers suggested to viewers they dedicated a sincere amount of time and energy to their channels, even outwardly stating the investment. Danielle told viewers, "I have been so excited that I have been on a roll. Third day in a row that I am filming a new video for you guys. And tomorrow I have another one," insinuating she had put a major investment into her channel, 
filming four days in a row. As the standard expressed by these influencers was to upload 0.98 beauty videos per week, Danielle proposed that she was providing extra time and energy investment on her channel. Natalie apologized for a lack of Halloween tutorials in 2016, noting time investment as an issue, stating, "I know that I only did three Halloween videos this year but those videos took a lot of time.” In one Halloween tutorial, she noted she spent five hours painting herself for the video and was awake until 2:00 a.m. finishing the content. Danielle mirrored these sentiments in her own Halloween tutorial, when she told viewers, "This is obviously something that is time consuming but trust me, when you're done, it's going to look phenomenal... I was actually filming this video like literally for more than 12 hours. Guys, no joke." Influencers never made mention of the investment as being too cumbersome, instead using the time element to suggest their dedication to viewers, through creating content viewers wanted to see and other influencers may deem too overwhelming to achieve.

The influencers also demonstrated investment by creating content viewers asked for, such as in an affiliate video by Natalie where she expressed she was creating this tutorial because, "I showed you at the end, like really sped up, at the end of my vlog and a lot of you requested to see [this] look. So I wanted to get [the tutorial for the look] up for you." As many viewers requested the tutorial, Natalie invested the time and energy to produce it, as an act of reciprocity toward viewer needs. This also functioned as a reciprocal act of involvement, as Natalie was invested in creating content to provide opportunity to the community. These actions suggested a demonstrated investment by influencers based on involvement with the community. However, viewer assessment of these measures ultimately can determine the overall impact of investment strategies. 
One way influencers also may extend their time and energy beyond their YouTube channel, to meet the emotional needs of viewers, was through physically traveling to new locations to engage in face-to-face meet-ups with viewers. During the videos reviewed for this study, Natalie traveled to New Jersey, New York City, Miami, and California to visit with viewers in planned meet-ups, often established by companies she had partnered with for video content. Natalie always gave viewers plenty of notice about a meet-up. She verbalized the date, time, and location of each meet-up, in addition to placing the information in the description box on YouTube and on her other social media accounts. Natalie also posted video content to her channel that showed her meet-ups and featured several viewers interacting with her personally. This act may be the highest level of reciprocity and investment, producing a great return on investment for viewers, as they are able to interact directly with Natalie, gain new information about her and further emotional bonds through pure face-to-face interactions.

Regardless, viewer assessment of investment was less prevalent than other social capital constructs. Some viewers provided superficial reference to the invested time and energy in creating videos, with one viewer telling Danielle, "I can tell you put a lot of effort into the video..." Even in a Halloween tutorial posted by Caitlin, where she opened the video with a oneand-a-half-minute long theatrical trailer showing the character she created, viewers commented only superficially on the effort taken in creating such a long introduction. One viewer told Caitlin her "editing skills [were] on point," while another stated, "I’m blown away by that intro!" Only one viewer suggested a reciprocal action for the effort displayed, stating, "Watches intro Instantly subscribes." There was no mention of how much time it took to create such an involved introduction, suggesting viewers were engaged with the content, not necessarily the influencer's overall investment. 
Interestingly, viewers were more apt to question perceived investment of influencers as being too low, either through the time dedicated to creating content, a lack of effort, or questions of personal motivation. As discussed, Natalie apologized for only uploading three Halloween tutorials in 2016, noting they took a long time to film and edit, suggesting they are a sincere investment to her community. One viewer actively questioned this investment, stating,

About how much time do you think it takes to film, cut, and edit? Would you say that it takes as much time as someone going to work from 9-5 Monday - Friday trying to get a project done for work? I'm sry [sic], but you do not have children, you do not have a set work time that you would get fired if you didn't meet, and you really don't have to be accountable to anyone.

This comment noted influencers can make their own schedules and are not subject to standard work practices, insinuating it should not be difficult or a major time constraint to film content, as it was their job and should be expected. Viewers also questioned investment to the community in terms of personal motivation to create content. One viewer noted, "I love you [Natalie] and I know you've been busy with life moving and what not but I can see it you are DEFINATLY not passionate about this anymore you use to be my favorite!!! But your almost emotionless..." This comment suggested there was a perceived level of work and life balance which must be maintained. If influencers do not post content demonstrating their work ethic, they are questioned about their investment. However, this comment also suggested that if work appeared to dominate their lives, viewers felt a disconnect to content through a lack of emotion.

This relates to findings that proposed balance was assessed by "the influence of the individual seeking balance on those around them and the influence of those around them on the individual seeking balance" (Reiter, 2007, p. 282). This suggests the expectations of viewers' impacted influencers' perceived social capital. Viewers needed influencers to invest time in their channels for bridging capital, as gaining informational resources was directly impacted by the 
amount of content created by influencers. If they did not create content, there was a void in the information available to viewers. Viewers also needed influencers to invest in their channels for bonding capital. Emotional resources are far more scarce in a community, as they require consistent offerings of time, trust, and reciprocity to achieve (Burke et al., 2011; Putnam, 2000). These viewers needed influencers to emotionally invest in their communities to provide these scarce resources. Thus, if influencers did not project their emotional engagement in addition to investing time in creating detailed content, there was a second potential void in capital, through a lack of emotional resources.

While some viewers suggested influencers did not invest enough time or energy into their channels, others defended their content, providing alternative reasons for why videos could appear to show lower levels of investment. One viewer placed the blame back on the community and suggested since this was the influencer's job, she had a right to distribute her investment based on the gratitude she received in return, noting,

Maybe she's getting burned out on the negative comments and that's why she might not be too enthusiastic about recording videos for ungrateful viewers. No one on YouTube owes anyone videos. Yes, it's her job, but you can quit a job if you don't want to do it anymore. You don't owe your job to anyone. It's definitely okay to comment out of concern, but some of you are bratty.

Other viewers supported these comments, stating, "If people don't like the content you've been putting out, they don't have to watch but I will ALWAYS drop what I'm doing or stay up an extra 20 min to watch [Natalie]." This disparity in opinions suggested investment was subjective and may be assessed differently across viewers. Based on the needs of the viewer, they may have been underwhelmed by a five-minute long make-up tutorial, as it could fail to produce the information they desire. However, others may be overwhelmed by a forty-minute long Halloween tutorial, as it would appear unachievable to those not willing to engage in such a 
time-consuming process. This further defined the notion investment by influencers was better assessed as a balance, where content was expected to have elements that demonstrated the influencer had given thought to the video and dedicated the appropriate amount of time to produce it properly, without being so overwhelmed they lacked the emotional resources required by viewers.

Investment, reciprocity, and social capital. As investment was based in action, assessed through time and energy (Portes 1998; Roberts \& Sherratt, 1998; Silverstein et al., 2002), it appeared to be a required activity by influencers. There was a perception of a baseline that influencer content had to meet to even be considered acceptable in terms of investment. If any of these influencers dipped below this level, viewers were quick to judge the content as less invested than usual. Additionally, when influencers went above-and-beyond to create content displaying extra time and effort to produce, such as videos with heavy editing and production techniques included, viewers tended to assess this at a superficial level. Instead of commending the influencer for providing additional investment in the comment, viewers focused on the overall impact of the video.

This suggested viewer perception of investment may be guided by preconceived expectations of beauty influencers, garnered through the social capital already existing in the community. As one researcher suggested, reciprocity was proportional to the initial investment in a relationship (Silverstein et al., 2002). In reviewing the influencers first videos, this increase in investment toward bridging social capital was apparent. Across all three influencers, their first videos were noticeably different from current content. All three videos were filmed in a single scene with no editing. Caitlin did not speak in her first video, while Danielle and Natalie spoke much more informally about topics than in their current content. The lighting made it difficult to 
see products and Natalie's video featured audible noise from the television in the background. The information presented did have impact statements included, such as telling viewers how to apply a lipstick properly to make lips look fuller or sharing examples of how an eye look could go from day to night by adding a new eyeshadow, however, the content was difficult to assess for credibility as it was the first video produced. Over time, as the influencers gained more notoriety, their content focused more on providing information in a coherent, actionable manner. This required a noticeable increase in the investment in content creation, such as removing distractions from the videos, editing content to remove mistakes, and taking the time to create content viewers requested.

As these three influencers reached substantial subscriber counts and continued to gain new subscribers each month, it was assumed the initial level of investment required for bridging capital had been met by these three influencers. Burke et al. (2011) suggested individuals initially may be drawn to digital content to gain bridging social capital, through gathering new sources of information. This assumption was apparent in this YouTube community. There was a level of investment expected by viewers of this content. If viewers did not deem the investment by the influencer to be great enough, they would not have been able to reach large subscriber counts, as reciprocity was based on mutual benefit for the parties providing resources (Blanchard \& Horan, 1998; Tayler, 1982).

In regard to bonding capital, investment was assessed across a different set of parameters. Bonding social capital was impacted by the exchange of emotional resources, such as affection and compassion (Putnam, 2000). When individuals develop bonding social capital, they tend to focus less on obtaining new, unique information and instead provide support between members, as emotional resources are typically scarcer in communities (Burke et al., 2011; Putnam, 2000). 
Investment returns tended to grow in magnitude with time and engagement (Roberts \& Sherratt, 1998). Thus, as communities developed stronger bonds and exchanged more valuable emotional resources, the act of investment was less rooted in pure information gain and more in the perceived dedication to the community overall. Time and energy no longer were based solely on the quantity of information presented but rather on the quality of the interaction.

\section{Social Capital Maintenance - Redefining a Model}

This research proposed digital social capital, in the YouTube beauty community, was generated through trust and reciprocity, which was impacted by five constructs: similarity, credibility, impact, involvement/engagement, and investment (see Figure 4).

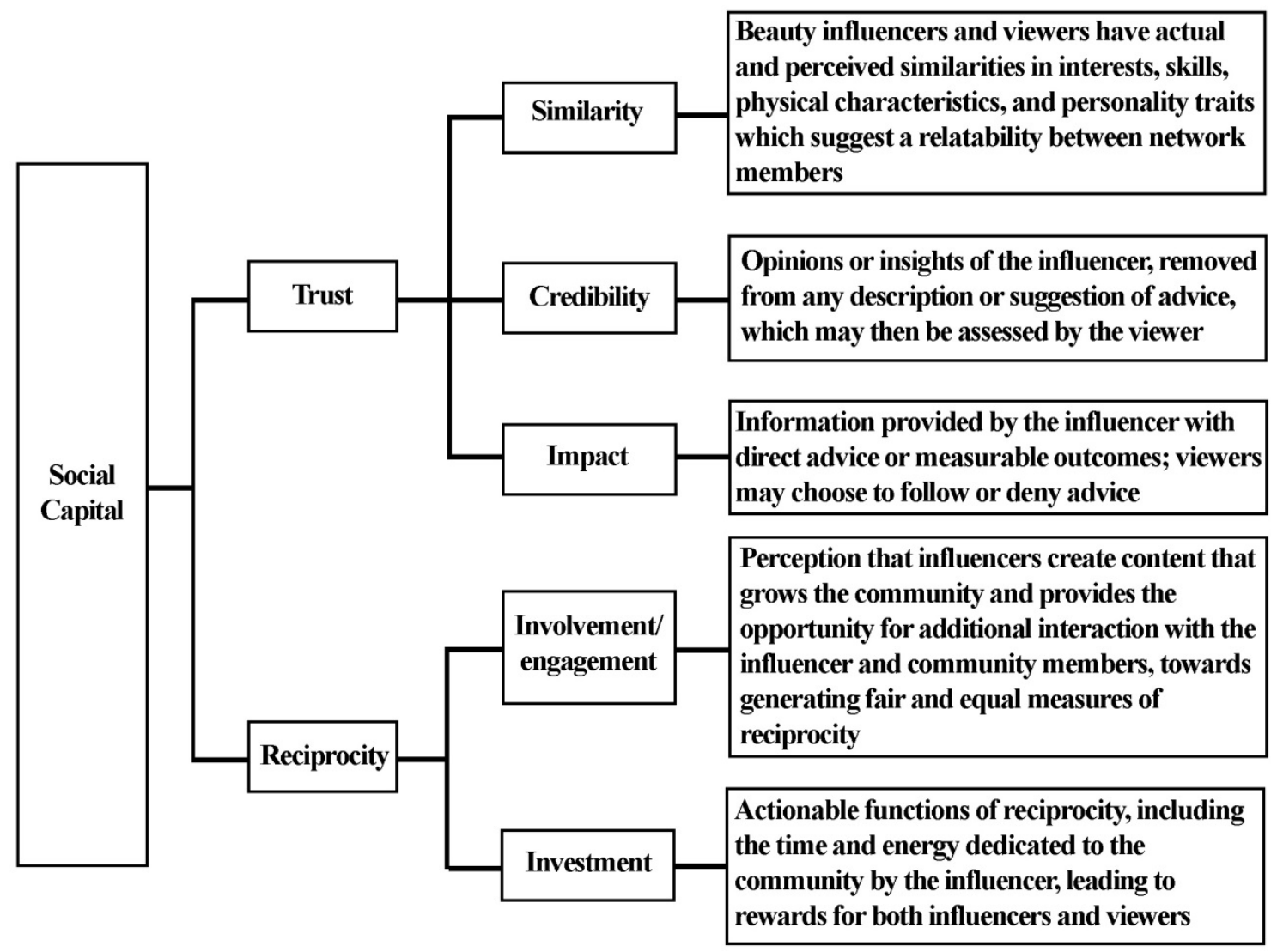

Figure 4. Redefined model of digital social capital generation and maintenance for YouTube beauty influencers 
This revised model features several key distinctions from the original model. The first key difference was the integration of two forms of social capital, trust and reciprocity. Maintenance of social capital on YouTube was more abstract, due to the presence of multiple relationships across a single community, which appear to be guided by the trust between members and reciprocity of their actions. These two tenants of social capital are at the core of determining how influencers maintain bonds in this digital community.

This research suggested trust functioned as the more abstract tenant of digital social capital, however, it was essential to maintaining bonds in the YouTube beauty community. Viewers based their reactions to video content heavily on their trust in the influencer, through similarity, credibility, and impactful statements. In regard to bridging social capital, trust in the information being presented was key to viewer response and interaction with the influencer. All three influencers projected measures of similarity, credibility, and impact deemed trustworthy and reliable by viewers. The major difference in information gain was displayed in sponsored content, in terms of credibility. While viewers may trust in the advice being presented, as it had an actionable component, when influencers suggested products or techniques in sponsored content, based solely on their opinions, viewers were quick to question whether the information was trustworthy or whether it was the effect of paid promotion.

This occurrence provides support to the notion bonding capital also was deeply rooted in trust. There actually appeared to be an inverse effect in regard to trust and bonding capital. Viewers who noted they had been members of the community for extended periods of time, were conflicted by sponsored posts, in terms of trusting the influencer. Some viewers noted sponsored content appeared contrived, as compared to non-sponsored or affiliate/free product videos, while others noted an emotional disconnect in these type of videos. Both of these reactions were 
products of time and engagement, in which influencers had promoted themselves in a certain way that viewers suggested did not match their sponsored posts. There was a certain threshold for how resources should be presented to viewers, mostly focused around content appearing genuine, personally-connected, and displaying emotion. When this was not met, viewers responded with questions of whether they could trust influencer content. Deviations from baselines for trust were not well tolerated in the YouTube beauty community, often based on the notion viewers expected more from influencers, in which they had set themselves to a standard which some content could not match. Even if the information being presented was diverse, there was still the commitment to the community and emotional growth that was missing, causing questions of influencer intent and trust.

The other form of social capital, reciprocity, was considered a duty as influencers are expected to produce content showing an exchange of both informational and emotional bonds. Deviations from this norm were not well-tolerated, as viewers had grown to expect a certain level of reciprocity from influencers. This reciprocity appeared to become more of a burden when influencers began to monetize their content. As the viewer on Natalie's video suggested, these influencers do not work a typical 9-5 job. Creating YouTube content was their job, from which they received direct revenue. Thus, when an influencer noted they have not created certain content based on time-constraints, it could be seen by viewers as a lack of involvement in their careers. Smaller YouTuber beauty influencers who cannot yet fully monetize their video content may be given reprieve by viewers for not posting content as often or having lower time investment in videos, as they likely have another job to supplement their beginning YouTube careers. In contrast, the three influencers in this study openly have noted YouTube was their fulltime career, suggesting they should put equal effort in their videos as one might in a standard 
job. If influencers post a single ten-minute long video once a week, the perception by viewers was they had not fulfilled the duty of their job. Even though there was time and effort put into filming, editing, and uploading content, it may not meet the involvement criteria expected by viewers, therefore questioning the influencers reciprocity to viewers. Reciprocity was seen as an expectation, as a form of social capital that must reach a certain threshold to be deemed fair and impactful among viewers.

While trust and reciprocity are created through key measures as adapted from Sander and Lee's (2014a) model, there are three constructs from their original model excluded from the revised version, based on each constructs disconnect from digital beauty influencers and their communities. Demographic data was included as a part of similarity of the influencer, operating under the notion of actual similarity. In this study, all three influencers engaged in projecting personal demographic information about themselves as a way to show actual similarities to viewers, such as similar ethnic backgrounds or age ranges. This community functions on a oneto-many relationship, with the influencer being the initial generator of digital social capital. Demographic data should be considered a sub-set of similarity, as the one influencer suggests similarities in demographics to many viewers.

Sander and Lee (2014a) also suggested activity, defined as people having an active or passive usage of the network - as a construct of social capital. In the context of this study, influencers must engage in active use of the network to promote themselves and their content. This would be considered an act of investment, as influencers cannot passively engage the network to remain involved in the community. For instance, when Natalie had a nearly month long break in her content, viewers noticed the passivity and commented on it frequently. It was discussed in such large quantities Natalie addressed the issue in a video and promised to return to 
actively producing content, as a way to remain engaged with the community. Influencers must remain active with their channels and involved with viewers to reap the benefits of social capital, therefore, this construct may better be categorized as a mandatory threshold, rather than a potential type of social capital.

Finally, the notion of benefits and advantages were omitted from this revised model. Sander and Lee (2014a) originally proposed social capital included benefits from the network, which only are obtainable through membership in said network. As a social network site, YouTube does not require any formal membership for viewers to interact with videos or content creators. Any individual may access a public YouTube video for informational, entertainment, or emotional purposes. As membership was not required on YouTube, any individual can potentially can benefit from resources and information, which suggests benefits are an overall effect of social capital, not a construct of it.

This research demonstrated social capital may not function in the YouTube beauty community like offline relationships. Influencers did not create content within a single type or form of social capital. This research suggested influencers project a variety of bridging and bonding social capital measures in each video, allowing viewers to assess the pieces that best fit with their needs at a given time. If a viewer was looking for information, she may focus in on the impact and credibility statements of an influencer. If a viewer was watching the content more passively, without needing to garner any additional information on the subject, she may be more willing to accept the emotional resources provided by viewers, such as feelings of affinity between influencer and viewer. These three women appeared to understand viewers with varying levels of connection to the community are accessing their videos. The need to create content that projected different types of social capital was evident in all three influencers' videos, suggesting 
they were aware of this continuum and sought to maintain capital across the community through aspects of trust and reciprocity.

\section{Authenticity and Beauty Influencers}

This potential strain on social capital ultimately was affected by a secondary aspect of influencer identity: the projection of an authentic self. As these influencers are individuals, they were assessed for personal authenticity. Yet, they also are functioning as brands, monetizing themselves based on the knowledge they are human beings. This positions them as being the person-as-brand, where they had to relate messages of personal and branded authenticity at the same time, developing an individual authenticity but also one promoting the brand as being real. These measures are not readily assessed across digital communities in which friendship and information sharing are connected to tangible forms of capital, such as monetary gains. This notion related to the second sub-question of this research: What practices are employed by YouTube beauty influencers to construct and maintain perceived authenticity?

As YouTube beauty influencers functioned as both the person and brand, they operated by generating authenticity marketable to the community at large. This notion was based on the experience economy (Gilmore \& Pine, 1999), which suggested economic growth lies in the value of experiences, not goods or services. Beauty influencers are direct players in the experience economy, offering their viewers an experience or transformation through their channels, not necessarily a tangible good or service.

Gilmore and Pine (2007) proposed the style of authenticity projected by those engaged in person-as-brand enterprises was influential authenticity. The concept of influential authenticity provided meaning to offerings and called individuals to a higher purpose (Gilmore \& Pine, 2007). The researchers specifically noted beauty was a form of influential authenticity as it was 
"the appreciation of qualities that give pleasure to the senses or spirit," in addition to noting community as another form of influential authenticity based on "a sense of unity with others around us and a general connection with other human beings" (Gilmore \& Pine, 2007, p. 91). To render authenticity of this type, influencers must provide meaning to their offerings, coinciding them with viewer aspirations and self-image.

Based on the notion meaning was central to consumers in influential authenticity, Gilmore and Pine's (2007) model of authenticity was applied to assess how viewers may experience the influencer's content as "[the person-as-brand] must earn the privilege of being deemed authentic only through the act of rendering" (Gilmore \& Pine, 2007, p. 90). One mechanism to render authenticity was to create a unique identity and establish where it falls in a real-fake matrix (Gilmore \& Pine, 2007; see Fig. 2, p. 41). The real-fake matrix functions within two key axioms: whether an offering was what it says it was and whether it was true to itself (Gilmore \& Pine, 2007).

Narratives have been crafted in this study through both the first-person accounts of the influencers and my interpretation as the instrument of research. These narratives give an account of how influencers defined themselves, through the interpretation of myself as a member of the community. The narratives served as a baseline for determining if influencers are what they say they are and whether they are true to themselves. Statements related to both measures from the model will be included in an initial assessment of the projected authenticity category by influencers, as Gilmore and Pine (2007) proposed this was the initial step for building an authentic brand.

Influencer initial authenticity categories on real-fake matrix. Based on the narratives crafted in Chapter 4, each influencer's projected identity was classified as being in the real-real 
category. As such, the influencers shared some similarities in the way they defined their initial authenticity. All three influencers suggested they chose YouTube as their medium based on the affordances it could provide compared to other social network sites. YouTube provided a way to connect with individuals in multiple different capacities. The opportunity to create content on YouTube was noted by influencers as being an "outlet," a way to express themselves visually, audibly, and textually within the community. At the time these three women joined YouTube, many other platforms were either in their beginning stages of development or had not yet integrated features of audio and video into their programming. YouTube offered these influencers the chance to broadcast themselves across geographic and cultural boundaries in a variety of ways.

YouTube also did not feature entry barriers established by other platforms. To watch a YouTube video, a viewer did not have to be a formal member of the site or the beauty community. There were no prerequisites to accessing YouTube content, other than having a digital connection to view the site. Influencers could project their information to quite literally anyone who wished to access it, not just those with their username. The search feature of YouTube did not require knowing an influencer's name or channel but relied on keyword searchers. YouTube information, therefore, could be broadcast on a larger scale to more diverse communities, extending its reach beyond those "in the know."

In regard to authenticity in all three influencers were provided the affordances through YouTube to do what they promised from the beginning of their channels. Natalie summed up their collective thoughts well when she stated her initial intention to begin YouTube was she, “just wanted to share [her] knowledge with [viewers] and connect with [viewers] and build online relationships..." 
Caitlin. Caitlin identified as being true to herself - fun and quirky - which she demonstrated through displaying humorous content in her videos, regardless of the sponsorship category. She showed herself dancing to music and making jokes about herself. She also expressed humor in her intentions with her tutorials to viewers, making statements such as, "If I'm having girls' night out or if we're going clubbing, which I never do, let me just not lie to you guys right now. But if I were to go clubbing, I'd definitely wear this look." Caitlin did not modify her expressions of similarity and personality across the three categories of sponsorship, producing 36 mentions in sponsored videos, 40 in affiliate/free product, and 33 in non-sponsored content. Caitlin's self-assessment was that she created content because she genuinely enjoys YouTube and the beauty community. She told viewers at the end of nearly every video she "love[d] them" and would "see [them] in the next video," suggesting she enjoyed creating this content and would continue to do so for them.

Danielle. Danielle, being true to herself, self-identified as a mother and wife, with beauty being her passion. She often directed viewers to her vlogs on a second YouTube channel, which featured candid footage of her family, allowing viewers a more intimate look at her personal life. Her passion for her family was featured on her beauty channel as well, where she has garnered partnerships based on her status as a wife and mother. This identity was replicated in videos across all three categories, such as showing how to achieve quick looks, targeted toward women with busy lives, specifically mothers. She told viewers in one video, "I am [just] a girl who loves beauty," and stated in another she "really love[s] filming make-up tutorials" as she "love[s] make-up," supporting her notion that beauty was a passion of hers, not just a hobby.

Natalie. Natalie's assessment of being true to herself was projected through her image as being down-to-earth. She shared several candid moments with viewers, such as showing herself 
singing to music and laughing at her mistakes. She frequently mentioned individual viewer comments, offering her opinions to questions viewers asked or referencing a funny statement left by a viewer on a previous video. In a non-sponsored video, she left the decision to fix a mistake in a video up to her viewers on SnapChat, suggesting she trusted their judgment enough to directly impact her YouTube content. Additionally, Natalie's assertion that she wished to connect with viewers and build online relationships was furthered by the fact she has met with viewers face-to-face, allowing the relationships formed online to translate to a physical connection.

Real-real authenticity projections. It was of great interest that all three beauty influencers projected a self-defined authenticity of real-real. Gilmore and Pine (2007) argued that projecting a brand (in this context the person-as-brand) as being real-real was the most problematic category to maintain as experiences involving commoditization almost always carry one fake element, such as self-centric motives. While Gilmore and Pine (2007) suggested positioning a brand as real-real can be difficult to maintain, these three influencers have chosen to do so. This could be explained by the person-as-brand status they sought to define.

These three influencers were actual human beings, functioning in a real world, where authenticity was considered a measure of being genuine and reflective of the true self (Kreber, Klampfleitner, McCune, Bayne, \& Knottenbelt, 2007; Henderson \& Bowley, 2010). As authenticity often was related to sustaining relationships and generating more meaningful bonds (Greenhow \& Robelia, 2009; Livingstone, 2008; Marwick \& boyd, 2010), there was the perception being realistic was advantageous in generating social capital and accessing new resources. Therefore, an individual functioning as a brand likely would wish to project herself as being real, as being true to herself and doing what she says she would be desirable human 
qualities (Baym, 2010; Gilmore \& Pine, 2007; Henderson \& Bowley, 2010; Marwick \& boyd, 2010).

Deviations from projected authenticity. The beauty influencer, functioning as a personas-brand, was defined authentically through the experience provided (Gilmore \& Pine, 2007). The person-as-brand posed an even greater risk when navigating between authenticity categories with no explanation. This followed the assumption if a person-as-brand situated herself in one authenticity category then later switched to another, the dissonance between the two measures may be interpreted as an act of inauthenticity by others, as the drastic shift may indicate an initial false identity (Gilmore \& Pine, 2007). Inconsistencies may be deemed inauthentic to viewers, based on the notion deviations should not be necessary if an identity was rendered as authentic. For example, when then chief executive of Abercrombie \& Fitch, Mike Jeffries, made comments about the brand being exclusionary, suggesting it sold only to "cool kids... the attractive allAmerican kid with a great attitude and a lot of friends," consumers were quick to note this did not align with the brand image projected by the company (Denizet-Lewis, 2006). While the misalignment was an initial issue, Jeffries sparked further inconsistencies when he issued an apology, stating,

...we care about the broader communities in which we operate and are strongly committed to diversity and inclusion. We hire good people who share these values. We are completely opposed to any discrimination, bullying, derogatory characterizations or other anti-social behavior based on race, gender, body type or other individual characteristics (Abcarian, 2013).

While the original comment brought about questions of moral and ethical debate, it was not a direct deviation from the brand's authenticity. The deviation occurred when Jeffries attempted to apologize and retract his original statement. Engaging in target marketing was commonplace in fashion retailing, albeit rarely discussed so bluntly. Thus, when Jeffries took his 
original statement and suggested the exact opposite in his apology, there was an inconsistency in the brand's authenticity. Consumers saw the brand as being fake, as trying to rectify the situation through presenting comments which were not true to the brand nor were they based on any actionable promises. There was inauthenticity in Jeffries' apology, shifting from his real feelings of exclusion to fake feelings of mutuality, suggesting one identity must be inauthentic due to the drastic deviation in thought.

While this was an extreme example, it did illustrate how deviations can affect the perceived authenticity of a brand and individual. Slight deviations may occur without penalty, as "no enterprise remains perfectly True to Itself or is always exactly What It Says It Is [sic]" (Gilmore \& Pine, 2007, p. 237). However, strong deviations from the authenticity model may be met with resistance. Great deviations suggested an inconsistency, especially when the influencer had positioned herself as being real-real, generating influential authenticity with viewers.

Gilmore and Pine (2007) proposed that within influential authenticity, there was a polarity between being sincere and insincere, as well as self- and other-focused (see Figure 5).

\section{Polarity of influential authenticity}

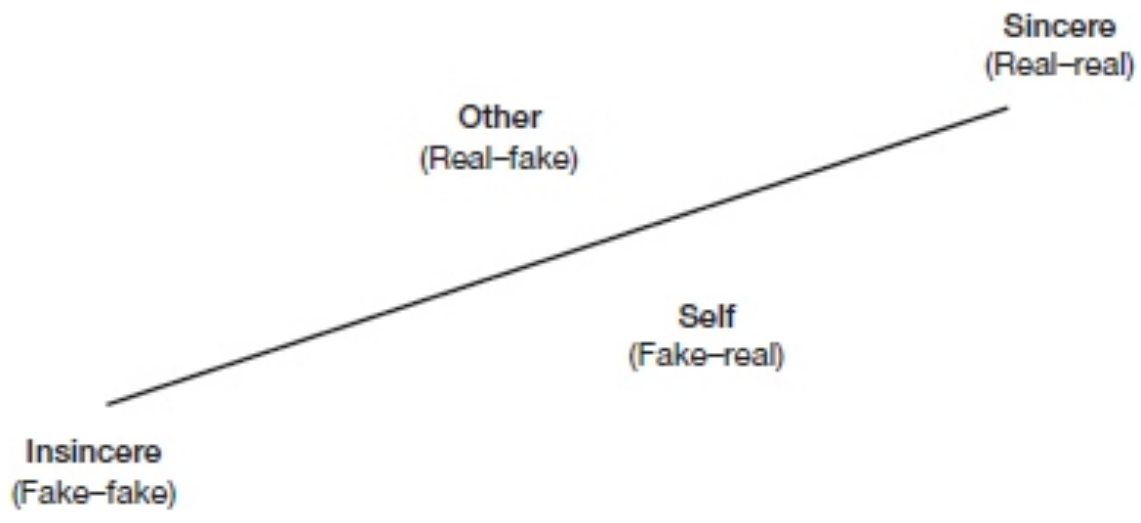

Figure 5. Gilmore and Pine's (2007) polarities of influential authenticity, based on deviations from projected authenticity 
The location at which an influencer fell on this continuum helped to define the type of authenticity she was projecting. Between the polarity, influencer authenticity was assessed from sincere to insincere, based on deviations from pure real and pure fake authenticity. Across the polarity, influencers were assessed as self-motivated or selfless, based on their actions and viewer feedback. I assessed each influencer individually for inconsistencies in authenticity projections, from which figures were generated based on deviations in authenticity. In the following figures (6-9), each marker represents a single instance of deviation. The markers were separated by sponsorship type, as indicated on each figure. If a marker includes a number, this represents the number of multiple cases within a sponsorship category producing at least $80 \%$ similarity in deviations, as assessed by my stance as the researcher-as-instrument or through similarity in viewer comments.

To be considered a significant deviation for this study, influencers had to project inconsistent claims directly to their established authenticity categories. The nature of this qualitative study required that I be the instrument of assessment. As a five-year member of the community in which this study was completed, I assessed deviations as a viewer and a member, based on past experiences with each influencer's video content, projected narratives by the influencer, and subjective assessments of the interpretations of those narratives.

Viewer deviations were assessed based on the top ten comments for each video. For an entire video to be categorized as a significant deviation, at least $50 \%$ of the comments had to address an inconsistency directly. Additionally, if a comment addressed a deviation and received one of the top three highest "like" values on a video it was assessed as an inconsistency. Receiving a high number of likes suggests viewers may share similar feelings without directly stating them (Chatzopoulou, Sheng, \& Faloutsos, 2010). 
The depth of the circles from the polarity line was indicative of their impact in terms of being self-motivated or selfless. Those circles farthest from the line illustrate more extreme deviations, while those closer to the line indicate less dramatic inconsistencies. The depth was assessed in two forms: (1) the number of times the influencer deviated from a projection in a video and (2) the viewer assessment of deviations in a video, including the percentage of comments directly addressing the inconsistency and popularity of that assessment in the form of likes. Markers also indicate the position of authenticity relative to a true real-real (sincere) and a true fake-fake (insincere). A marker placed directly on the line indicates a deviation away from the real-real authenticity projected by influencers not impacted by self-motivated or selfless actions, but rather was based on inconsistencies in the two axioms of authenticity: whether an offering was what it says it was and whether it was true to itself (Gilmore \& Pine, 2007). The combination of these factors depicted deviations from the original real-real authenticity, as defined by narratives constructed through both first-person accounts and research assessment.

Caitlin: Polarity of influential authenticity. Among the three influencers, Caitlin projected similar disparities in her authenticity across all three sponsorship categories (see Figure 6, p. 169). 


\section{Deviations from Projected Authenticity - Caitlin}

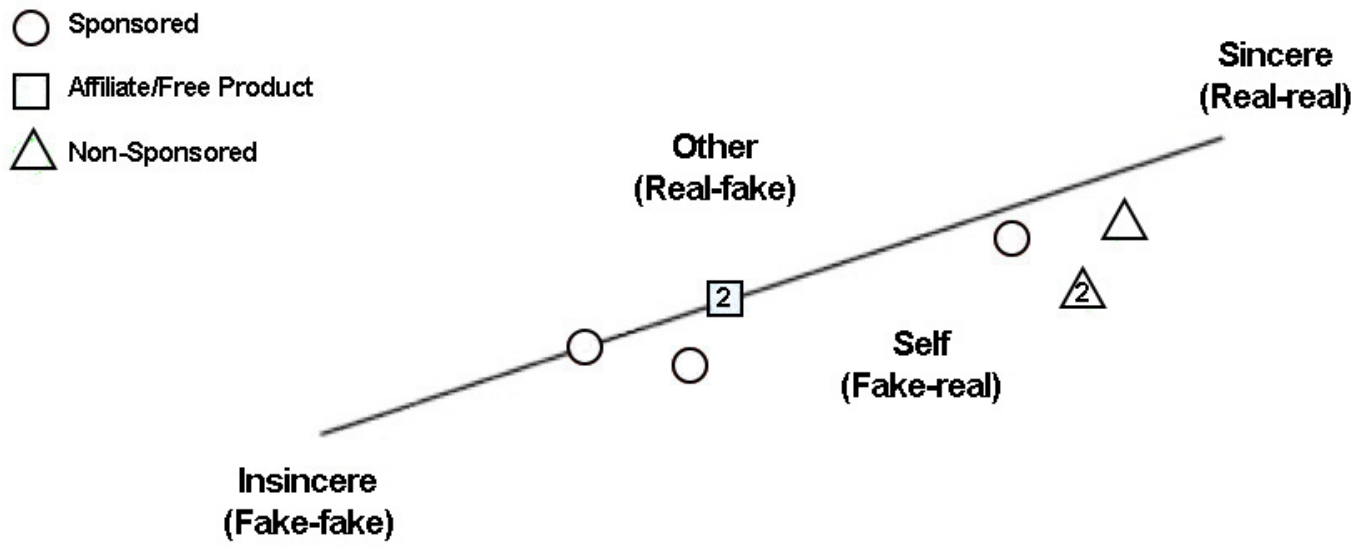

Figure 6. Polarities of Caitlin's authenticity, as defined by deviations from projected real-real authenticity

Caitlin produced a single instance where she deviated from projected measures of authenticity directly relative to non-sponsored content. In a "highlighter challenge," Caitlin noted the premise of the challenge was to create a full-face make-up look using only highlighter products. She clearly discussed this in the beginning of the video. However, throughout the video content, she frequently used non-highlighter products to bring the look together, such as a matte finish foundation and concealer. For individuals who were seeking out a true highlighter challenge, this does not fulfill the action of being what it says it is. Caitlin's desire to create a look appropriate for her needs, caused her to focus on a self-centric approach to this tutorial, creating a look true to herself but not what it claimed to be.

Viewers noted a similar disparity in another non-sponsored video, which was supposed to be an "everyday fresh glowing makeup tutorial." One viewer suggested, "It's not really "fresh", it's more like glam gorgeous look for me." This comment suggested viewer-feedback similar to the disparity in Caitlin's highlighter challenge. The viewer proposed the intention of the video was not met with the final product, as Caitlin had produced a "glam gorgeous look" rather than one "fresh [and] glowing." Similar to the previous example, this was due to a deviation toward 
the self, where Caitlin suggested a look was focused on what her perception of fresh and glowing was, rather than assessing how others in the community may define those terms. This pushes her authenticity projection away from the polarity line, toward being more self-focused than directed toward others. An influencer producing content based on her own definitions or interests would be considered more fake - a self-centric act - where pure realness would focus on the selflessness of content generation.

Additional deviations were witnessed in Caitlin's affiliate/free product videos. In a video labeled as a celebrity-inspired make-up tutorial, Caitlin spent the first minute and half of the video showing viewers how to use affiliate products, such as a leave-in conditioner, which were not related directly to the make-up tutorial in any way. The top comment and third most liked comment on this video directly addressed the fact Caitlin had demonstrated how to use these hair products in a make-up tutorial, also noting she was wearing a wig in this video and did not need to use hair care products on it. These instances suggested viewers questioned whether she was being sincere in suggesting these products, as she did not provide the same amount of information or enthusiasm for them as non-affiliate products. Caitlin portrayed what she promised to show in certain aspects of the video, however, the contrived moments of showing affiliate products made her appear unlike her normal self.

The greatest question of Caitlin's authenticity occurred during a sponsored make-up tutorial for a drugstore brand. The video itself did not feature any noticeable elements of deviation, but viewers were quick to call attention to questions of Caitlin's sincerity with the content. Caitlin included the appropriate FTC disclaimer for her sponsored content in this video, however, she did not verbally note it was sponsored, nor did she include any indication of this in 
the title of the video. Viewers questioned her judgment in not telling them directly about the sponsorship, suggesting the content may not be sincere since it was a paid post.

Caitlin demonstrated a total of eight deviations from her initial projection of a real-real authenticity, moving her authenticity toward the fake-real category. The deviations were not deeply motivated by aspects of the self. Based on frequency, distribution was split evenly between sincere and insincere. Tt was suggested Caitlin's authenticity category should be redefined as fake-real, due to these inconsistencies in Caitlin's projections and viewer assessment.

Danielle: Polarity of influential authenticity. Danielle's deviations were more centered around questions of authenticity primarily in regard to sponsored content (see Figure 7).

\section{Deviations from Projected Authenticity - Danielle}

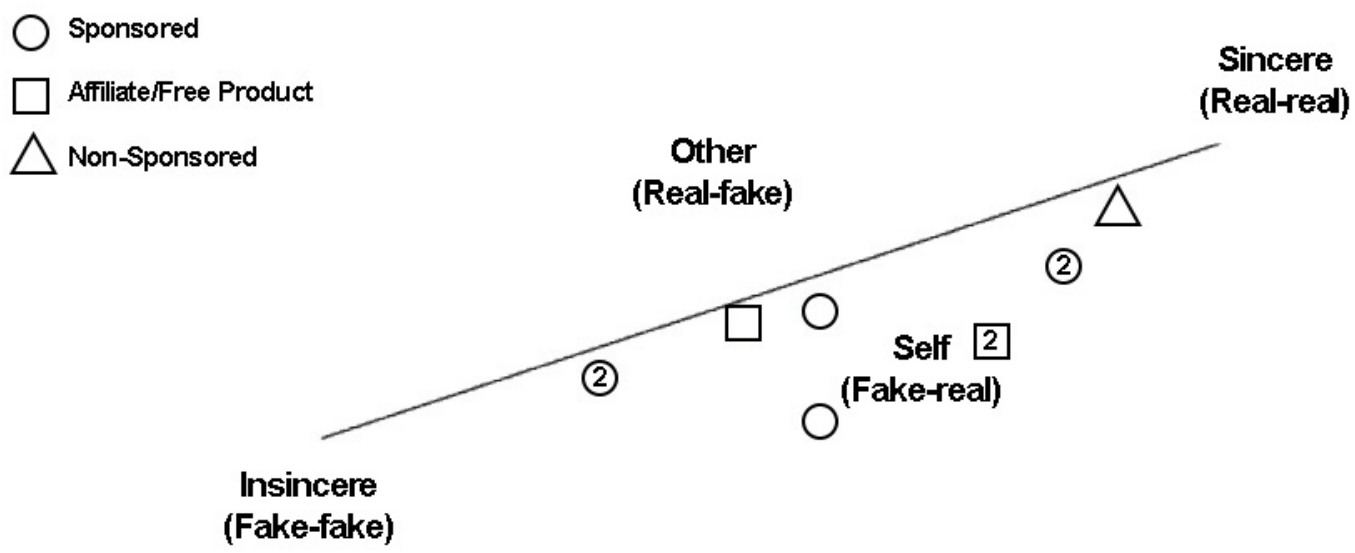

Figure 7. Polarities of Danielle's authenticity, as defined by deviations from projected real-real authenticity In Danielle's non-sponsored content, there was only a single deviation, which was assessed through a viewer comment. In this video, Danielle showed viewers how to achieve “effortless waves," however, one viewer commented "it doesn't look effortless. too many things to use, you are better off if $u$ wave it with a flat iron." This viewer assessment directly opposed the reason for the video, suggesting this tutorial did not do what it claimed to. The same viewer 
did not see this tutorial as effortless and therefore did not consider the content to be relevant to her needs.

Danielle's affiliate/free product content produced additional deviations from her initial projection of a real-real authenticity. In the lowest rated video on Danielle's channel, she told viewers she was going to review and demonstrate a new set of liquid eyeliner markers. Danielle proceeded to show the markers to viewers, providing swatches of the colors. Instead of demonstrating how to use them on the eyes, she used them in the coloring book. She suggested this was to determine how pressure might affect the color, stating,

Alright so I want to take a stab at actually using the markers as markers, since I did get a book for it. I want to show you because it might be more visible to see how much of a change can occur just by the amount of pressure that you can apply to the tip of the brush.

While these were proposed as being marker-style eyeliners, at no point did Danielle demonstrate using the product for its intended purpose, which was to line the eyelid. Therefore, viewers were not able to assess the actual use of the product, suggesting this video did not fulfill its promise to viewers. As the term demonstration was included in the title, viewers expected a look at how the product performed for its intended purpose. Since they were not given this information, they deemed the content confusing. One viewer called it the "most cringy moment ever" while another asked, "How is this a review/demo if you didn't apply them on your eyes?" These comments support the notion this video did not do what it claimed, however, there was no question whether Danielle was being true to herself. The content did not seem insincere, as viewers were more apt to question the intent of the video rather than the honesty with which it was produced.

The greatest question of Danielle's authenticity, though, was apparent through her sponsored content. Three of the five videos in this category featured deviations from her initial 
projection of real-real authenticity. One viewer questioned Danielle's sincerity in creating this type of content, stating, "i dont know if it's just me, but I feel like she does videos only with partnerships..." This viewer continues to note she understands the need for influencers to engage in sponsorships for profit, however, "it's just that [YouTube] nowadays is all about sponsors, but it would be nice if you just from time to time make a video just with your own products and by your own decision." This comment received 34 likes and was the top comment on the video, suggesting several other viewers agreed with her assessment.

This comment directly addressed a key concern among viewers in sponsored content, namely, was this the true feelings of the influencer or was she impacted by the incentives of paid content. With Danielle making statements such as "I can't live without [a sponsored product]" and then not showing that product in updated content, viewers questioned whether she was truthful in her appraisal. When influencers integrated sponsored products into their daily routines, it appeared as though the product truly was a favorite of theirs. When they made reference to a brand multiple times prior to sponsorship opportunities, viewers responded more positively overall. There was an established relationship between the influencer and the brand, suggesting she would recommend the product even if it was not through paid content. In contrast, when influencers mention a product or brand a single time and do not justify their claims, it seems as though the information may not be sincere. There was no justification over time of a relationship with the brand, which situates the content as being more self-motivated based on monetization, rather than true information gain to the community. It appeared as though viewers considered this sponsored content was less sincere, pushing it more strongly toward the fake end of the polarity. 
In total, Danielle demonstrated ten deviations from her initial projection of a real-real authenticity. All ten deviations moved her authenticity toward the fake-real category, as many were focused on elements of the self. The distribution between content deemed sincere (real) and insincere (fake) was close but inconsistencies were deemed neutral to sincere overall. Based on these inconsistencies in Danielle's projections and viewer assessment, it was suggested Danielle's authenticity category should be re-defined as fake-real.

Natalie: Polarity of influential authenticity. Natalie's deviations produced the most dramatic results of the three influencers, suggesting multiple inconsistencies in her authenticity projection (see Figure 8).

\section{Deviations from Projected Authenticity - Natalie}

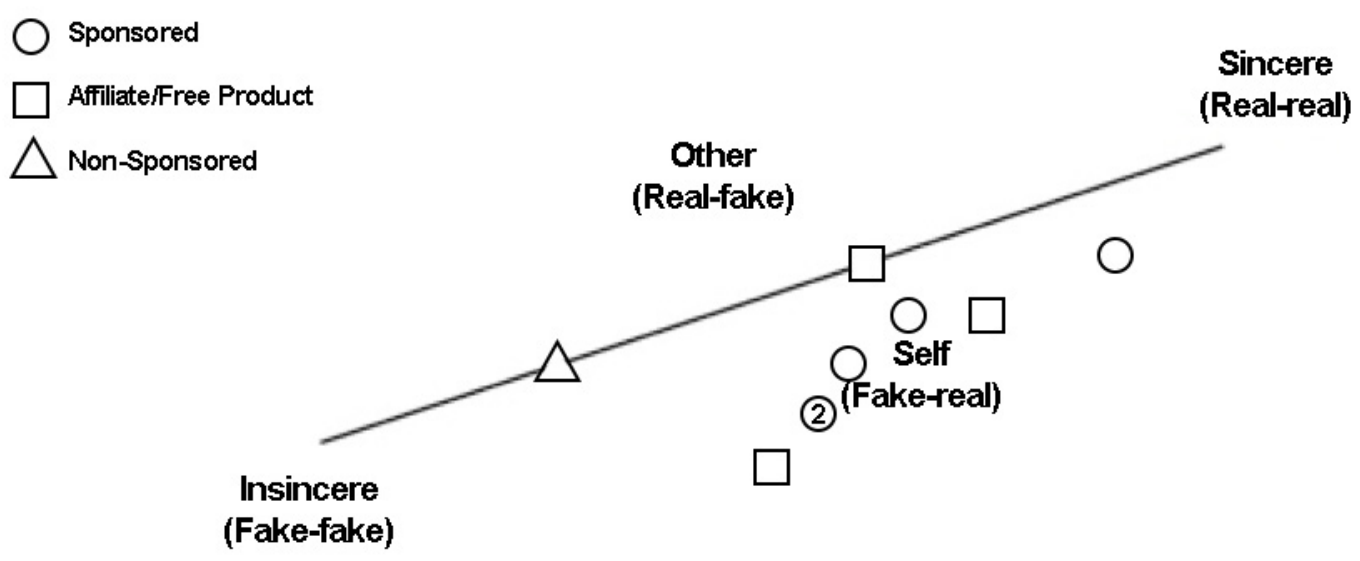

Figure 8. Polarities of Danielle's authenticity, as defined by deviations from projected real-real authenticity Natalie's non-sponsored content was assessed as inconsistent by viewers in a single instance. In her kids' makeup challenge video, she made an editing mistake in speeding up her voice to sound like a small child. She did not change the content when it was uploaded to YouTube, stating she did not notice the error until after the video went public. Sixty percent of the comments questioned her choice to not change it, with one viewer suggesting, "The helium voice sound effect is kinda funny since its a kids make up challenge but the fact that you didn't 
'notice' until after you uploaded it, kinda makes you seem like you're lazy about your work." Viewers were less concerned with the voice error itself, instead choosing to focus on how the decision to not re-film made Natalie seem less concerned about her role as a beauty influencer. The viewers questioned whether Natalie was being true to herself and to viewers, if she was producing content because she wanted to or because she felt like she had to. This moved the deviation strongly toward the insincere polarity, but it did not move from the line vertically as it was not motivated by self or other interests.

In both her affiliate/free product and sponsored content, all of Natalie's deviations were assessed by viewers. Across both categories, the predominant question of Natalie's authenticity centered around the amount of sponsored posts she engaged in and her commitment to the beauty community. One viewer noted she only put effort into a video because it was sponsored. Another viewer even suggested, "I can't even finish watching the video, there isn't much point in watching a tutorial that you learn nothing from." These comments suggest the content displayed in Natalie's affiliate/free product and sponsored videos was due to her monetary gains, often displaying information that was not what was promised to viewers. One viewer noted,

Stop complaining about the hate [Natalie] gets. These "hate" comments are loyal subscribers that are giving their HONEST opinion. Not some hater coming just to be an [expletive]. [Natalie]'s videos aren't same anymore. that's a fact. All of her videos lately are sponsored lately. that's a fact. It's okay that some people are upset.

Each of these instances questioned Natalie's authenticity, based on whether she was being true to herself and viewers, in addition to suggesting video content was not fulfilling what it had promised to do.

Another predominant question of Natalie's authenticity centered around whether she was truly interested in creating YouTube content, with viewers suggesting she no longer had a 
passion for the beauty community. One viewer noted, "I've always enjoyed watching your vids, you were always vibrant seemed happy but now.. call me crazy but you just seem off, like you don't really wanna 'be here' or whatever." A second viewer echoed this sentiment, stating, "I love you [Natalie] and I know you've been busy with life moving and what not but I can see it you are DEFINATLY not passionate about this anymore you use to be my favorite!!! But your almost emotionless..." Viewers noted Natalie personally did not seem as interested in creating content as she once had been and questioned whether she was uploading videos because she truly had a passion for the community she was in or if there were other motivating factors. One fan even suggested, "If your not happy or passionate anymore then why still do this?" There was a projection among viewers that Natalie's content seemed forced and her personality had changed, suggesting she was not being true to herself.

In total, Natalie demonstrated nine deviations from her initial projection of a real-real authenticity. All nine deviations moved her authenticity toward the fake-real category, as many were focused on elements of the self. The distribution was centralized as being between real and fake based on the polarity, however, it was strongly pushed toward element of the self. Based on these inconsistencies in Natalie's projections and viewer assessment, it was suggested Natalie's authenticity category should be re-defined as fake-real.

Fake-real authenticity in practice. Upon assessing these deviations by influencers, it was suggested their authenticity category be defined by the fake-real parameters (see Figure 9, p. 177). 


\section{Deviations from Projected Authenticity - Combined}

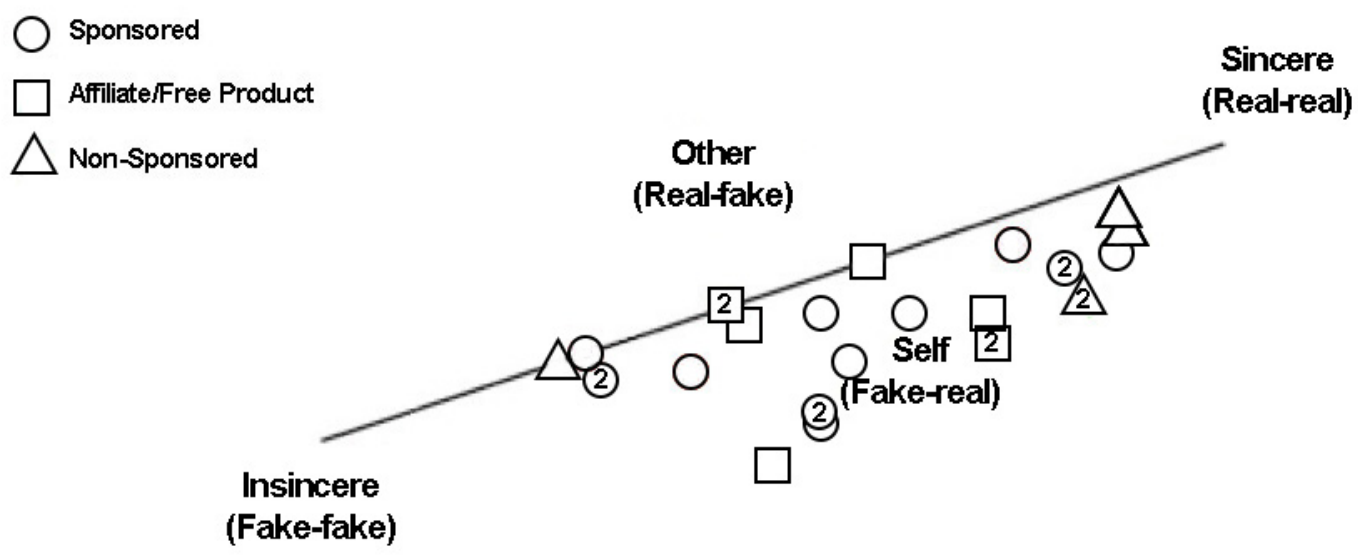

Figure 9. Combined polarities of beauty influencer's authenticity, as defined by deviations from projected real-real authenticity, suggesting proper placement in a fake-real authenticity category

In the fake-real category, authenticity was assessed by being true to the self but not being what it says it was (Gilmore \& Pine, 2007). These influencers were all true to themselves in the majority of their videos. They self-identified as beauty-lovers, members of a community which enjoyed utilizing beauty as an external form of expression. All three influencers were noticeably excited by beauty products and new techniques in their content. They filmed content true to their personal interests and passions, creating an interesting paradox in terms of the self-centric aspect of influential authenticity.

In creating content defined by their personal interests and talents, influencers are in turn pushing their authenticity into the fake-real category, through producing videos based on constructs of the self, not others. Across all three influencers, there were several incidents in which content was defined differently between the influencers and viewers. An influencer may say a make-up look was "fresh and glowing," however, viewers may not assess it as so. Influencers can say they are completing a 1960s-inspired hair tutorial, yet without defining what that means, interpretation was left to the viewer. The focus on the self also was displayed through products, with influencers suggesting products to viewers based on their personal life 
styles. Influencers tended to have a stronger focus on their selves and how they could extend those interests to their viewers, without having to change their personal routines and skill sets.

The most prevalent and consistent examples of the fake-real authenticity was projected in sponsored video posts. In these videos, influencers engaged in direct product placement and promotion of sponsored brands, which often was met with resistance. Viewers were quick to question the motivation and sincerity in sponsored content when influencers were not fully explicit about partnerships or engaged in subsequent sponsored posts. All three influencers abided by FTC laws for sponsored content, clearly listing the sponsorship with the appropriate labeling in the description box. However, viewers also suggested they preferred when influencers mentioned the sponsorship verbally, as well as in the title. Across all three influencers, viewers were more receptive to sponsored content when influencers were open about discussing their partnerships. These videos returned more positive comments and higher ratios compared to videos where only the FTC disclaimer was included. As the transparency of influencers increased, viewers were more receptive to their sponsored content, noting they understand YouTube was a source of revenue for influencers and applauding them for their success in partnering with their favorite brands.

As transparency decreased viewers were quick to comment that influencers were not being honest and sincere with them, suggesting they were trying to push products rather than engage with the community. This lack of transparency was demonstrated in all three influencers sponsored content. This was further clouded when influencers are less transparent about their desire to monetize their content. It was well-known by viewers that influencers utilize the site to generate revenue, however, they seem to appreciate sponsored content more when influencers are willing to admit they have partnered with a brand or company to create a paid video. 
Sponsored content typically was seen as insincere by viewers, with the few instances at the sincere end of the polarity being content where influencers identified their partnerships openly with viewers. The self-fulfilling nature of sponsored content alone suggests a benefit only for the influencer, through direct monetary gain, while products may not truly be a favorite of the influencer, rather a careful act of product placement. When coupled with a lack of transparency, these instances can produce feelings of insincerity and dishonesty among viewers.

A second instance of perceived insincerity by influencers was suggested when they engaged in multiple sponsored posts at a single time. Caitlin was the only influencer to post significantly less sponsored videos than the other categories. She staggered the sponsored posts throughout the year which lead to fewer questions of her authenticity based on her investment and engagement. In contrast, Danielle and Natalie both posted multiple sponsored videos within short time frames, leading viewers to question whether they were truly committed to the community or if they were being insincere, focusing only on the monetary gains available. One viewer commented,

Well since you now partnered with yet another company, and therefore have "updated" your makeup routines, I guess one of the next videos is a hair video about how you have "updated" your hair routine and have found yet another product, which have saved your hair even more than all previous products from other partnered companies have ...

This comment illustrated while viewers understood YouTube was a source of revenue for beauty influencers, they would like to see more personal, engaging content mixed in with the sponsored posts. When influencers posted multiple sponsored videos at one time, it created the illusion they were only interested in their monetary gains, rather than investing in the community with their emotional resources.

This research suggests individuals interested in pursuing the role of YouTube beauty influencer should position their authenticity as being fake-real from the conception of their 
channel. In influential authenticity, having a fake element does not indicate negativity, instead it suggests influencers may have their content better received if they admit it was self-motivated and self-guided, while helping the community through their personal interests and skill sets (Gilmore \& Pine, 2007). To be real-real, influencers would have to be truly pure in their intentions, which was nearly impossible with monetary gains. As influencers continue to monetize their content, there likely will be some form of personal motivation to create videos which are engaging and increase their viewership statistics. Beauty influencers should be true to themselves, allowing viewers to gain from their emotional attachments and perceived similarities, however, influencers also must be realistic about their motivations. If they do intend to utilize YouTube for monetization, they need to be upfront about their fake-real authenticity, being transparent about sponsorships and paid content, as well as notifying viewers of their selfmotivations. This will enable them to establish a stable fake-real authenticity and remain true to their image over time, rendering authenticity through showing viewers their content was true and meaningful, rather than saying they are being real.

Real-fake matrix and the person-as-brand. Beyond establishing practices for beauty influencers to render an authentic image and identity, this research also suggests Gilmore and Pine's (2007) model was applicable for the person-as-brand individual. While the real-fake matrix has been assessed theoretically across a variety of service industries, research had not yet addressed its practical application to individuals who function as a brand, such as beauty influencers or bloggers. This model measured elements of individual and brand authenticity simultaneously as it relates to rendering an overall authentic identity. The model uses concepts traditionally relegated to assessing an individual's authenticity, such as sincerity and selfmotivations, in a way that can then be applied to branded products, services, and experiences. In 
the context of this study, beauty influencers integrated their statements and content in a way that rendered authenticity both individually and through their personal brands. They connected with viewers emotionally and generated functioning relationships, while still creating a personal brand image.

The balance of both personal and brand authenticity had not yet been fully addressed in literature on authenticity. The notion of creating an identity which was both authentic and targeted may be seen as a paradox, as the two do not categorically seem to fit together, yet was required of YouTube beauty influencers. If influencers wish to gain tangible resources from the beauty community, often in the form of economic capital, they must assess how to balance the person-as-brand element in regard to authenticity. Researchers have suggested inauthentic individuals and brands are questioned in a mediated context (Gilmore \& Pine, 2007; Marwick \& boyd, 2010), so it was integral to understand if and how beauty influencers can project themselves as being authentic to determine the longevity of such approaches in this community. This research suggests Gilmore and Pine's (2007) model can assess this person-as-brand status, suggesting ways in which influencers can render an authentic identity while still being true to themselves and doing what they promise. This model works in a practical setting, as demonstrated by these influencers, if they can properly identify their initial authenticity category and produce content which remains true to the authenticity they have rendered.

\section{Dynamics of the Person-as-Brand}

Influencers have fulfilled a key tenant of Gilmore and Pine's (2007) authenticity approach in rendering an image based on their true person-as-brand status: they did not have to tell viewers they were authentic, since they had shown them. This raises a question on the potential limitations of positioning a human being as a brand. 
Brands are static entities, carefully controlled to reflect consumer needs and emotions (Aaker, 1996; de Chernatony, 1999). A brand identity takes an average of five years to craft, built on factors such as personality, positioning, and reputation (de Chernatony, 1999). The building of a brand is a business decision, an opportunity to showcase their product or service as being beneficial to the consumer and wanting to align with their self-image. Yet brands themselves are intangible. A consumer can touch a product, but they cannot touch a brand. They can talk to entities of the brand but not the true brand itself. Brands exist in reality in a much broader sense, while human beings are directly engaged with the lived environment.

Humans are much more complex, emotional, dynamic beings. They feel real emotions, such as happiness or pain. They communicate with each other in a variety of facets and have a tangible body that can experience the senses. Humans can hug each other, laugh together, and share emotional bonds. These influencers may be branding themselves for monetary gains, however, they are still humans. When consumers leave bad reviews about a product, the brand has a damaged reputation. When a viewer leaves a hurtful comment about an influencer, she does not only have a damaged reputation but can feel the emotions attached to the comment.

When assessing the person-as-brand approach, it was important to remember influencers are both a person and a brand. While they may position themselves as a brand for monetary gains, they are doing so through their humanity. Their identities are guided by humanistic intentions, which are developed far sooner than those in most brand identities. These influencers know who they are and what they have to offer. Their status as a human was not seen as a pitfall for the brand but rather a core component. When utilizing the person-as-brand approach it was important to remember human beings are dynamic and their identities will change over time. This push-pull between influencers being a person and a brand brought attention on how 
monetizing human interactions can be difficult and potentially draining, as the stricter notions of a brand identity confine the freeness of the human spirit. When assessing ways influencers can render their authenticity to brand themselves and other entities, it was important to remember that the influencer was a human being first, then a brand.

Brand identity and impact of influencers. When Aaker (1996) proposed brands could build a strong identity through innovation, by identifying patterns to clarify, enrich, and differentiate their brand through various perspectives, he suggested one means to do this was to classify the brand as person. By anthropomorphizing the brand individuals could relate brand aspects to their own self-images, often mirroring brand personalities to their own (Aaker et al., 2004; Fennis \& Pruyn, 2007). This was demonstrated in brands such as Nike, which promoted activity through their products, suggesting an individual buying Nike products also would be interested in engaging in an active lifestyle. Aaker's (1996) model of brand identity proposed the brand could function as a person by engaging in brand-customer relationships. Customers can speak to entities of the brand, both directly and indirectly, through various interactions such as engaging with a cashier at a store or asking for product recommendations on social network sites. The brand functioning as a person can be seen with human qualities, which then may suggest a type of self-congruity to the consumer (Aaker, 1997).

Beauty brands could substitute the act of defining a brand as a person with the person-asbrand representative, replacing anthropomorphized characteristics with actual individuals. Instead of focusing on creating an illusion of human connection, beauty brands can benefit from utilizing the bonds formed between beauty influencers and viewers. In doing so, brands are provided an outlet to connect their products or services to human characteristics and conversations based on real bonds in the beauty community. 
This section addresses the ways in which brands interacted with beauty influencers, through non-sponsored, affiliate/free product, and sponsored content. It discussed the different mechanisms used for branding in each section, as well as viewer response. A final recommendation for the person-as-brand influencer in regard to brand identity is provided, in the context of Aaker's (1996) model of brand identity.

Beauty influencers as the person-as-brand. While utilizing the person-as-brand as a feature of brand identity was intriguing to brands, they had to be careful to align themselves with the appropriate individuals for their products or services in addition to determining how to best situate the influencer in relation to the brand. Beauty brands may select influencers to represent their brands in a variety of ways. By better understanding how beauty brands define brandinfluencer relationships, this research then could address the possibilities and limitations of such relationships in the beauty community. If members were not willing to accept the branded content, the damage to the social capital and authenticity of the influencer may counter the influence brands wish to gain in these communities. Distrust of the influencer's motives could potentially lead to a distrust in beauty brands, through association. Exploring how viewers receive brand-related activities helped to address concerns over how branded content can impact the community's relationship with influencers, addressing the question: How do YouTube beauty public members respond to brand sponsorship activities through the comments section offered by YouTube?

This research suggested brand interactions with influencers functioned in a cyclical nature, as demonstrated in Figure 10 (p. 185). 


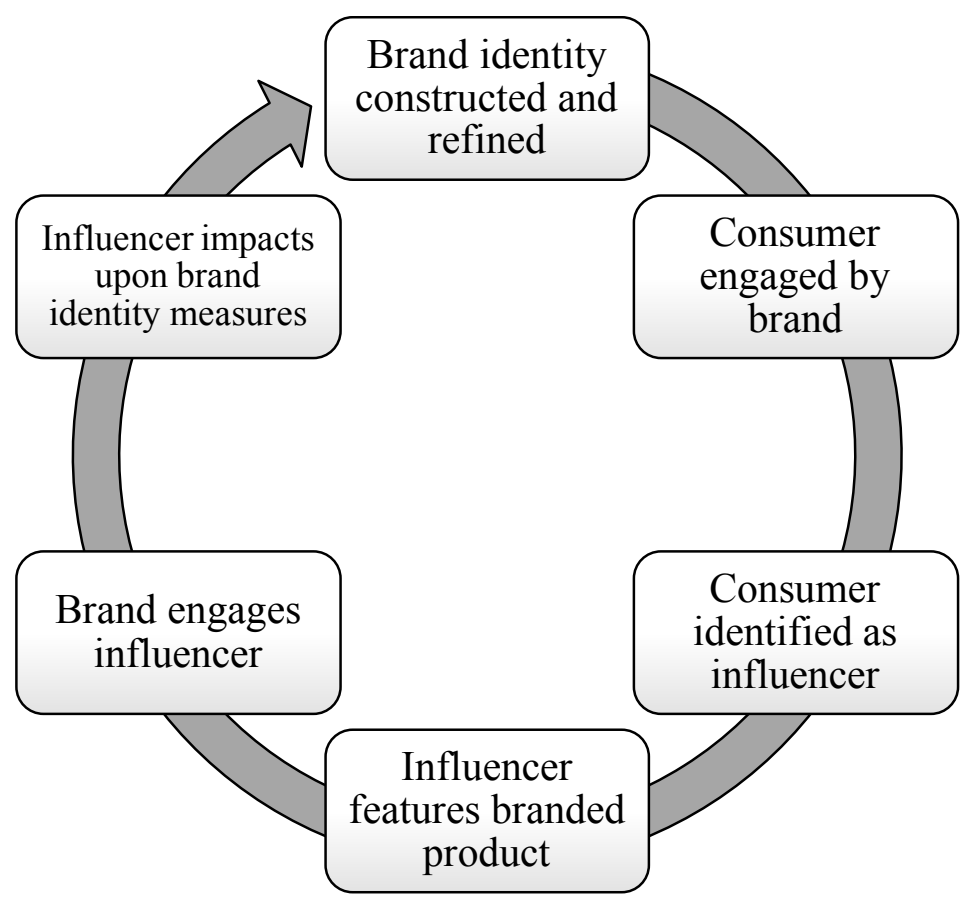

Figure 10. Cyclical nature of beauty influencer's impact on brand identity of beauty brands.

The process by which brands integrated influencers ultimately was defined by the three types of content (non-sponsored, affiliate/free product, and sponsored), as well as how influencers engaged with the products. Most relationships between brand and influencer appeared to begin with the influencer as a typical consumer, who has been engaged by the brand identity. Once the consumer identified as a beauty influencer, her status within this niche market compelled brands to deepen the relationship, through affiliate/free product and sponsored content. This act ultimately culminated with the beauty influencer impacting the brand identity of beauty companies, both directly and indirectly. Brand identity was redefined through the interaction with beauty influencers. The following areas further address this cycle and how it related to brand identity.

Non-sponsored content and brand identity influence. Separate from any paid branding opportunities by beauty companies, influencers also were customer elements of the brand, through their non-sponsored content. They were not paid directly by brands to produce specific 
content, nor were they receiving the products for free. Instead they were acting as a consumer of the brand, as are members of the beauty community. When an influencer purchased a product with her own money, she was engaging in direct consumption of the good as a consumer. When creating non-sponsored content that mentioned specific brands or products, influencers could connect products back to their needs and express their opinions, separate from any noted affiliations with the company. A rise in her status and notoriety in the beauty community allowed her opinions about the brand to be spread across a larger breadth than most word-of-mouth marketing (Tolson, 2010).

While this may appear to be a gamble to beauty brands, it actually provided a unique source of honesty and transparency to the brand, which was deemed important by consumers (Aaker, 1996; Aaker, 1997). For instance, Caitlin produced a non-sponsored video in which she compared drugstore to high-end make-up brands, showing viewers products she considered similar in color and finish. She recommended several affordable make-up brands as being comparable to more expensive products, suggesting viewers purchase these products as they are more cost-conscious. Viewers appreciated her review of the affordable make-up, agreeing the products looked nearly identical and stating specific brands were "amazing" for their ability to generate similar results to higher-end products. Natalie suggested a specific brand of face paint to viewers for Halloween make-up, stating they were easy to use and noting she had used them for previous tutorials, suggesting she had continued experience with the products. Viewers commented on her expertise in using these products and suggested they too may be interested in purchasing these goods to achieve similar results. In non-sponsored content, influencers frequently mentioned brands and products they considered good quality based on their experience and expertise. Interestingly, the brands in this context had no control over whether 
influencers enjoyed their products, as influencers purchased the products as a consumer, with no financial incentive from the brand.

This suggests in non-sponsored content, influencers relied on the initial brand identity which was furthered by their exposure to the products being offered. Brands cannot rely solely on influencers to create an identity, as Aaker (1996) proposes there are multiple elements to a brand identity, such as the organization, symbolism, and the products themselves. Brands benefit from influencer engagement with their products in non-sponsored content through a more passive approach. If a beauty brand has built a strong enough identity over time, influencers act as consumers, identifying with their products on a personal level, often based on self-congruity with brand image and identity.

While influencers are functioning as consumers, initially relying on the brand's identity to formulate their opinions, their communication reaches farther than the traditional word-ofmouth consumer experience could. The status an influencer has achieved in the beauty community through perceived social capital and authenticity, provided notoriety among viewers, often situating them in expert positions where viewers trusted their opinions. In all fifteen nonsponsored videos, influencers made at least one recommendation of a product based on their expertise. Caitlin suggested "one of [her] all-time favorite powders" to viewers, as "it makes [her] skin look so flawless." She noted she had been using it for an extended period and was comfortable labeling it as one of her favorite products of all time. When Danielle suggested a lotion from a specific brand based on her expertise with it helping dry skin, her knowledge of the product and status in the community encouraged viewers to believe in the brand as well. Trust was generated between the influencers and viewers, enabling brands to benefit indirectly from non-sponsored content. If an influencer enjoyed a product and recommended it to viewers, based 
on her personal experience with a brand's identity and product, then viewers considered this enough information to purchase the product themselves. This gave beauty brands the potential to gain new consumers through this indirect relationship to the influencer.

Affiliate/free product content and brand identity influence. One interesting way brands shifted focus from their brand identity to how influencers aligned with the product was through engaging in affiliate/free product relationships with influencers. Brands may choose to send influencers products for free or provide them with affiliate trips, predominantly as an incentive to someday engage in direct content generation with the influencer (Wu, 2016). These affiliations or free products were offered with no guarantee the influencer would mention them to viewers, as it was not paid content and therefore carried no content requirements by the influencer. Influencers were able to utilize the products or services as they saw fit, with brand intention being they would show products to viewers and provide a subtle type of product placement. Danielle illustrated this in an affiliate video where she let viewers know she was sent a product for free but was not told to make the video:

So they did send this to me but I am not being paid to do this video. I was not told to. I get things, I am so grateful for that, I get things all the time to my P.O. Box or my house so I have that awesome blessing of trying out different skincare products and makeup.

In all fifteen affiliate/free product videos from this study, influencers engaged in some type of direct product mention, even though it was not required in affiliate/free product content. This placement came in various forms. Influencers sometimes highlighted new products in tutorials, discussing their positives and negatives based on first impressions. For example, influencers used a new product in a tutorial, then told viewers their initial reactions, such as "The [eyeshadow] colors are super pigmented and like so creamy and beautiful," and "This is an illuminating primer, as you can see, my skin is already glowing. If you feel like your skin is a 
little dull, it's gonna brighten it up." These products were not featured in any subsequent videos, requiring viewers to rely on the trustworthiness of the influencer's initial reactions.

In other instances, influencers featured a single product across several videos, noting its importance in their beauty routine. Natalie engaged in this with a lip mask, applying the affiliated product and discussing its benefits to viewers across several videos. The consistency suggested this product was in fact a favorite and had worked its way into her daily make-up routine.

In some cases, influencers chose to film an entire video about free products they received. Danielle engaged in this three times, once in a haul video where she showed public relations packages sent to her by brands and twice again in a video dedicated to new products from her favorite brands. She noted she had been sent the products for free in both instances with no commitment to review them by the brand.

Influencers engaged in varying types of product placement, without brand direction. When free products were no payment was received by influencers for engaging with the products, thus it was truly at the discretion of the influencer whether or not products were featured in videos. This engagement by brands was focused on creating more active relationships with influencers.

This raises an interesting question of ethics in regard to accepting free products or affiliations from brand, especially in a medium where sincerity and trust was valued highly by viewers across a variety of communication methods. The discussion of ethics in free product promotion has been researched in terms of blogging, primarily by fashion bloggers (Abidin \& Ots, 2015; Archer, Pettigrew, \& Harrigan, 2014; Marwick, 2013; Sedeke \& Arora, 2013). Researchers found fashion bloggers view free product promotion in different ways. Some 
bloggers suggested mentioning products were provided for free was the ethical thing to do, but they should remain true in their product appraisal (Marwick, 2013). Certain bloggers even let brands know they may receive a negative product review if they send free products deemed ineffective by the blogger (Marwick, 2013). Others felt a sense of commitment to both their viewers and the brand, as they wanted to be upfront with viewers but retain beneficial monetary relationships with brands (Abidin \& Ots, 2015). One blogger navigated this divide by highlighting free products in their best light, such as saying a see-through dress was airy and a good layering piece, therefore not directly lying to viewers about the nature of the product but also providing a positive appraisal for the brand (Abidin \& Ots, 2015).

While it has not yet been researched empirically, this research suggested beauty influencers prescribe to the second category of blogger ethics by providing opinions of products in a way both superficially honest and in support of the beauty brand at the same time. When assessing affiliate product videos, there was not one instance where influencers stated they did not like a free product provided to them. Instead, influencers often justified their promotion of affiliate products by engaging with them in demonstrations or reviews to show their abilities. These justifications were not always deemed appropriate by viewers. When Danielle was testing out a free face mask, she recommended the product because she thought it tightened up her smile lines, proclaiming,

Oh my gosh, I just noticed something guys. So this here if I relax my face [points to her smile lines]. So I have like really strong lines right here and this is like the first area of my face where I notice some changes as far as getting older and they're always so visible and I feel like they make me look older because it's so pronounced and I feel like it tightened up this area. Look you can barely see it and usually when my face is relaxed you can see these lines from my smile, my smile lines, basically is what it is. These creases that happen here. So this tightened up my face because they are not as pronounced and visible as they usually are and I'm so like 'Oh my gosh, this actually works! 
While Danielle suggested the product worked well and was worth the $\$ 75$ price, viewers suggested, "I don't see the difference..." and another noted, "I actually rewinded the video just to see if there was a difference. I'm sorry but you look the same." Some viewers did not see how the product worked in the ways Danielle suggested. One viewer even accused the product of being “a bit gimmicky" and was not sure Danielle's tutorial "justifie[d] the price." However, the majority of viewers commended her for the in-depth tutorial. Her appraisal of the product was short and somewhat masked by the length of the overall tutorial, focusing more on the product itself rather than her opinion of it. The act of embedding opinions quickly and discretely into videos reviewing free products allowed them to engage with both the viewers and brands, suggesting a branded product while not making it seem overtly obvious to the viewers.

Influencers engaged in similar acts when they suggested an affiliate or free product, then contradicted their statement. Caitlin suggested viewers use an illuminating primer, which she received through a brand affiliation, to "look nice and glowy" underneath their foundation. Just one minute later in the tutorial she suggested a foundation she was "obsessed" with to go on top of the primer to create a flawless finish because the foundation was "very, very matte." The foundation was not an affiliate product. Caitlin also did not mention the glow she received from the primer, instead focusing on how the matte foundation made her look like she had a new layer of skin.

This relates back to the fashion blogger in research by Abidin and Ots (2015) who suggested a way to retain relationships with both viewers and brands was to be honest but place the brand in a positive light. Caitlin was known for enjoying full coverage, matte foundation, which she mentioned in several of her videos. Viewers have come to know her for being an expert at applying foundation that masks the skin underneath. When Caitlin suggested the 
affiliate product to viewers as it would make them look glowing and fresh, then covered it with a matte foundation, she was engaging in an act similar to bloggers. She suggested the positive of a product from a brand, then created content honest to herself. The primer may not actually have been her favorite or ever used again, however, the quick placement maintained a relationship with the brand. This masking of products behind relatable content and favorites was a common theme among influencers in regard to affiliate and free products.

These results suggested influencers utilized affiliate and free product videos as a means of maintaining relationships with viewers and brands simultaneously. Influencers were rarely questioned by viewers for their appraisal of free products, as they typically were masked behind more engaging content. In doing so, they were able to place free products from beauty brands in a positive light, fostering brand relationships and the potential for future sponsorships by the company. While the ethics of such activities is questionable and should be addressed in future research, this appeared to work well for influencers. They were able to engage with both of their audiences - viewers and brands - at the same time and foster relationships which were advantageous from multiple perspectives.

Sponsored content and brand identity influence. Once the beauty influencer had gained notoriety and a standing in the beauty community, brands appeared to feel it beneficial to engage in direct relationships with the influencer, positioning her as being an entity of the brand itself. Sponsored content was one of the closest relationships a brand could have with an influencer, as the company was paying the influencer to produce content related directly to the brand itself. The only relationship stronger than sponsoring content was a brand actually working with influencers to collaborate on or design a product, however, these instances were not actively discussed by influencers during this study. In this research, influencers engaged in two different types of 
sponsored branding: (1) product placement through indirect video content or (2) content dedicated to one brand.

In the first and most prominent instance, sponsored products made a featured appearance in a video that otherwise showcased non-sponsored content. Caitlin demonstrated this in a makeup tutorial. The first nine minutes of the video showed viewers how to achieve a smoky eye look and contoured face, without any direct mention of the sponsored brand or product. At the nineminute mark, she showed a red lip color to viewers and noted "The part I have been waiting for this entire video because I am so excited about these new lippies...” The lipstick belonged to the brand who sponsored the video. She told viewers the "shades are gorgeous" and then continued to give product information, such as how they were comfortable to wear for long periods of time and that the product provided "intense rich color of a lipstick" while it "glides on like a gloss." The product was featured for over a minute, in which she shows herself applying the lipstick and then promoted the product as being superior to others for being "the first of its kind." This short snippet in the video featured direct product placement, where the sponsored company had provided her with the product and paid for content to highlight it. While the entire video content did not focus around the one lipstick, Caitlin created a specific look meant to showcase the sponsored product, which she then commended through its placement in the video. This was extremely common among all three influencers, with at least $60 \%$ of influencers' sponsored posts containing this type of sponsorship.

A second type of sponsorship illustrated a more direct brand relationship, where influencers created content focused solely around a specific brand. Danielle demonstrated this when she partnered with a hair care company to demonstrate their products and suggested them to viewers. She opened the video with a monologue about how she used to loathe her curly hair, 
telling viewers it was unmanageable. She then noted she loved her curly hair now because she knew how to care for it properly and suggested the sponsored brand's products had changed her perception of her hair. She suggested specific products to viewers from the brand, demonstrated how to use them and provided detailed information about the product's benefits for curly hair. The entire video focused around her hair and how these sponsored products helped her personally and suggested to viewers how they could have similar hair to hers if they purchased these goods. The video featured one brand and the accompanying products, with content that was related directly to the goods shown to viewers. This type of sponsorship was less common among influencers, with only six occurrences across all three influencers.

These sponsorships draw this research back to a discussion of ethics and integrity within the YouTube beauty community. Sponsored content was a direct advantage to the influencer, providing them with monetary gains in exchange for their endorsement (Wu, 2016). Marwick (2013) found that if fashion bloggers crossed a threshold it could lead to question of the bloggers ethics and authenticity. One blogger in Marwick's (2013) study described this threshold as being the difference between "you" and an actress.

Beauty influencers built their followings by presenting themselves as the 'everyday woman.' They wanted to make others "feel beautiful" and share their talents, so every viewer had the opportunity to engage with beauty as she felt comfortable. They frequently explained to viewers "Make-up is fun. It's art. It's a way of expressing yourself" and implored them to share their creations with others. They created a community based around the notion they are similar to viewers, they are all beauty lovers, and the community was a safe place to express their interests. Each influencer was unique in her approach, yet similar in the role she played in helping viewers to define their personal self-concept (Krampf \& Ueltschy, 1997; Tolson, 2010). 
This raises the concern over whether engaging in directly sponsored content was in fact considered an ethical act in the beauty community. It was assumed any beauty influencer engaging in a sponsored post was going to speak positively of the brand, as they are being paid to promote its products or services. A negative promotion would damage the relationship with the brand and decrease the opportunities for future partnerships, leading to less monetary gains. However, engaging in sponsored content also placed the influencer as being closer to the actress character Marwick’s (2013) participants describe.

When engaging in direct sponsored content, influencers shifted from being personal brands to entities of an external brand. Viewers relied on influencers, both in terms of social capital and authenticity, to provide them with trust and reciprocity. Viewers expected a level of honesty in the resources being provided to them, in exchange for their membership in the community. If an influencer actively promoted a brand as being fantastic, without a solid knowledge of their products or services, they risked losing credibility with viewers - in essence selling themselves out for the sponsorship. This was demonstrated by Caitlin in a video created through a sponsorship with a drugstore brand. Viewers directly questioned why Caitlin would engage in such a sponsorship, as they deemed the content to be contrived and suggested the products she recommended were not high quality. Viewers saw this as duplicitous as she had chosen her allegiance to the brand rather than to them.

When influencers engaged in sponsorships with companies they had recommended to viewers in the past, separate of any sponsored affiliations, the response was much more positive. Viewers actually commended influencers for being sponsored by brands they had built a reputation for recommending, such as when Natalie engaged in a sponsorship with make-up superstore Sephora. This was seen as an achievement for Natalie by her viewers, as she had 
actively promoted the store in the past and had built up its reputation through her non-sponsored and affiliate content. The sponsorship was not seen as an opportunity for pure monetary gain but rather as an achievement for building her personal brand successfully in the community. In order to combat questions of ethics, this research suggested influencers should engage in sponsorships with brands with whom they have built a reputation with, both among brand ambassadors and viewers.

Similar to affiliate/free product content, the ethics of sponsorships among beauty influencers will require additional research to further assess its impact in the community at large. However, this research offered an initial understanding of how influencers engaged in these sponsorships and how viewers received them. As viewers seek trust and reciprocity from influencers, in addition to assessing their overall authenticity, engaging in sponsored content could be a gamble. Influencers should engage in the same transparency in their sponsored content as they do in other videos, choosing only to create paid content for brands they truly stand behind. Viewers preferred sponsored content to be a celebration of influencer achievements rather than an obvious pushing of products. Honest content produced the greatest results in terms of sponsorships. Influencers have built their communities based on being a real person, not a celebrity endorser, and should uphold this even through their sponsored content.

Embedding the person-as-brand into the brand as person identity element. All three influencers engaged with influencing brand identities across the three sponsorship types in varying capacities. While the relationships may be considered passive or active depending on the type of engagement, the prevalence of influencers engaging with products or services in their content suggests the overall identity of a brand can be impacted by its connection to the influencer. 
The nature of the YouTube beauty community was unique in that its outreach and depth redefined many traditionally held tenants of how brands sell to consumers (Aaker, 1996; Aaker 1997; de Chernatony, 1999). This does not hold true for just beauty communities, as research has suggested influencers across a variety of channels can impact brand identities through their connection to niche markets (Tolson, 2010). When assessing how brands may create and maintain their identities when engaging in partnerships with digital influencers, this research suggested for beauty brands, it would be beneficial to embed the person-as-brand influencer into their brand as person identity element.

By utilizing the person-as-brand in their identity building, beauty companies were able to attach a physical identity to their products. They had no need to rely on anthropomorphizing goods as a means to assume a similarity to consumers, as beauty influencers had actual similarities and relationships to viewers. The influencer's in this study built an identity through perceived social capital exchange and authenticity projection, as previously discussed, which over time established them as being impactful in the YouTube beauty community. If a brand feels their core personality aligns with an influencer, it would be beneficial to attach their products to her. Instead of focusing on the imagined characteristics a brand might possess, this association provides a real face and personality to the brand.

The personality projected by the influencer was passed onto the brand, rather than the brand personality being adopted by the influencer. Caitlin was still fun and quirky, Danielle was still personal and succinct, and Natalie remained focused on being down-to-earth and relatable. These posts suggested if the influencers truly enjoy these products and are willing to be direct representatives for them, then the personality of these brands must align with their own ideals. Caitlin was energetic and fun, just as a red lipstick could be perceived as a bolder statement, thus 
aligning the individual with the product. Through product association, if Caitlin was fun and enjoys wearing bold lip colors, then the brand which has sponsored her also must be interested in creating fun products to match her personality.

In addition to personality measures, Aaker's (1996) model also proposed the brand as person element was defined by brand-customer relationships. In the YouTube beauty community there are established relationships formed between individuals in this community. The depth and variety in the relationships may vary, however, their existence alone was of interesting note to brand's looking to situate the influencer as an entity of their brand. Brands are able to tap into the notion that influencers are seen as friends and advisers to community members, through their perceptions of bridging and bonding social capital, as well as their established authenticity with the community. The brands themselves do not have to explain anything to the viewers, as they have no direct connection to these pre-existing relationships. By functioning outside of the already established bonds, they can situate themselves as a third party, offering a good or service without directly affecting the relationships which already exist. The brand's role essentially was de-emphasized in these communities, as the brand-customer relationship dissolves into the influencer-viewer bonds that already existed.

Reach of the person-as-brand in relation to brand identity. This research suggested the person-as-brand be embedded into the pre-existing brand as person element of a brand identity. From embedding an individual into this established framework, the influencer may absorb some of the constraints brands face in establishing personality and engaging in brandcustomer relationship. However, determining the level at which influencers should be integrated into the brand identity framework was important for beauty brands. 
Influencers have built their communities through engaging with niche markets (Tolson, 2010). Beauty influencers are no exception to this. While the beauty community is expansive, it is still a niche market with specific needs and interests. Not every individual who purchases beauty products engages with this community, suggesting there are unique factors which draw viewers to this community, such as the perceived social capital gained by being involved. These factors should be assessed by beauty brands before engaging in relationships with beauty influencers, as any potential damage to the influencers credibility based on brand relationships could in turn harm the overall reputation of the brand.

This research does not suggest beauty brands should replace facets of their brand identities with influencers but rather utilize them as a source of insider status. Beauty brands should engage in creating a solid brand identity. They should establish themselves through the product, define the organization, position themselves as humanistic, and provide heritage through brand symbolism (Aaker, 1996). The company should have a clear focus on what it wants to provide and to whom. Viewers showed greater positivity toward sponsorships which made sense for the influencer's personality and needs, primarily based on their history of using a brand's products and overall expertise. This suggests beauty brands can benefit from allowing the influencer to establish the relationship. Influencers should be utilized as a tool to add personality and communication elements to the brand-as-person feature. Influencers should not be seen as the solution to creating a humanized brand identity.

Creating a brand identity is integral to company's success, telling who the brand is and what it stands for, a relatively static definition of the brand (de Chernatony, 1999). In contrast, human beings are dynamic, engaged in relationships requiring several re-defining moments over time. These influencers are a direct part of that engagement. They do have the ability to reach 
niche markets and impact upon pre-existing bonds in the community. They also must show their loyalty to the community which provided them with this influence. If the beauty communities on YouTube did not exist, these opportunities would not be available directly to influencers.

Therefore, influencers must navigate the careful line between gaining monetarily and remaining a loyal member of the community, a push-pull in choosing between the self and others.

This research showed brands should utilize influence as the person-as-brand in a way that makes sense for all parties involved. The influencer should be a reflection of the brand and viewers, just as the brand should reflect similarities to the influencer. For instance, when Natalie partnered with Sephora, viewers were excited for her. She had created several non-sponsored Sephora hauls prior to this video, throughout several years on her channel. She frequently mentioned shopping in Sephora and showed their products in her videos. The sponsorship appeared authentic to viewers. Natalie truly recommended shopping at Sephora to viewers, not just due to a sponsorship, but because she had experience with the brand and believed in its reputation. Beauty brands can benefit from tapping into digital communities through influencers, as long as they are cautious of the origins of the community and the bonds which have been formed. If viewers feel betrayed or question a partnership, it can adversely affect the credibility of the influencer and ultimately the brand. However, if viewers trust this partnership was honest and directly relates to the influencer's character, then beauty brands can reap the benefits of gaining tangible personality traits and brand-consumer communication through the influencer's role in the community.

Branding potential by beauty influencers. This research suggested beauty brands were able to situate themselves in a unique position in the YouTube beauty community, serving as an external factor directly impacted by influencer-viewer bonds, both passively and actively. Beauty 
brands can engage with influencers in a variety of facets, which does not always require them to directly sponsor influencer content. Through passive means of non-sponsored posts and more active engagement in sending influencers free products, beauty brands are still impacting their overall brand identity by aligning themselves with these impactful influencers. In an effort to reduce the dissonance between imagined brand as person traits and real person-as-brand associations, beauty brands should consider embedding the person-as-brand into the brand as person element in their brand identity. In doing so, brands still are able to tap into the personaspect of their brand identity but through more direct means. These brands are able to tap into more personal influencer-viewer relationships through the person-as-brand affiliations and convert aspects of the influencers personality to their own brand identities. In doing so, beauty brands can access a new niche market and influence their brand identities through the actual associations of those related directly to the consumers they desire to reach.

While brands and influencers may be the two parties directly engaged in these interactions, viewer feedback had an impact on how these relationships were viewed. Across the three sponsorship categories, viewers had differing responses to the levels of brand-influencer interaction. Overall, viewers were more compelled to engage in consumption of beauty brands when the presentation by influencers appeared to be genuine. This typically was assessed as a function of time, where the relationship between influencer and brand was built through frequent interaction and demonstration of a branded products impact in an influencer's beauty routine. The ability of viewers to comment on these relationships did impact influencer content, as they engaged in sponsorships less frequently than other content and crafted their messages more carefully in these videos, based on viewer response. This suggests the community as whole has 
an impact on brand-influencer relationships and should be regarded as important in the communication of these messages.

\section{Message Reception through Digital Communication Channels}

The commenting feature of YouTube allows for digital communities to engage in twoway communication. Instead of only allowing the influencer to project her social capital, authenticity category, and relationships to brands, the comments section served as a means to assess viewer response to these constructs, as the receiver of communication. In word of mouth communication, findings proposed the sender was more likely to be engaged when there was satisfaction with the communication being presented, such as influencers monetary gains or generation of emotional resources from community members (Sweeney, Soutar, \& Mazzarol, 2008). Similarly, the receiver was more likely to act on sender communication if they already had a good opinion of the resources being provided (Sweeney et al., 2008). This was demonstrated by influencers and viewers in the YouTube beauty community.

Message reception among influencers and viewers. In regard to the YouTube beauty community, sender-receiver communication was impacted by the resources and relationships established on the site. The benefits of the sender based on these mechanisms have been discussed, however, receiver response was assessed in more detail to determine if the potential for a feedback loop existed in this community. If receivers can impact sender messages, through their support or denial of communicative practices by influencers, there is the potential for their feedback to have future impact on the ways influencers communicate with viewers.

Non-sponsored content and viewer feedback. Across non-sponsored content, viewer feedback was positive and supported the communication presented by influencers. Among the 15 videos in this category for the three influencers, 13 featured comments of at least $70 \%$ positive 
response and support for influencers. Based on Madden et al.'s (2012) framework (see Table 1, p. 76), these comments were predominately focused around information gains, expression of personal feelings, and impressions. Viewers utilized the comments in this category to comment on the information provided to them, as well as request further information from influencers.

One viewer to Caitlin's channel asked, “\#thatglowtho. love this look, i've never seen anyone highlight so close to the eyes, is there a reason you did that?" Another viewer told Danielle, “Wow! This makeup look came out so good!! What dollar store is this, [Danielle] lol?” Viewers used this category to request future video content from influencers, such as a request on Natalie's channel asking, "Can you please do a video on your top 20 Anastasia single eyeshadow. I want to purchase some but not sure which ones are the best."

Non-sponsored content also produced many impression-based comments, which Madden et al. (2012) define as,

express[ing] people's immediate reactions to what they have watched in a video or read in comments. They tend to be very short, sometimes even single words, and will generally be fragments as opposed to complete sentences. They will often contain exclamation marks and paralinguistic features like emoticons, as well as interjections like "Yay!", "Boo!" and "Wow!" (p. 706).

Viewers had more superficial reaction to this content, expressing their enjoyment in simple phrases. They were more likely to comment single phrases like, "You are beautiful!" or "This is so dope! So different \& creative!" indicating their initial reaction to the content. As compared to opinion-based comments, these appear to be immediate reactions to what they have viewed and are based on their surface level appraisal of the content. These comments were mostly positive, offering the suggestion that the content was deemed appropriate in both initial response and prolonged engagement. 
The feedback in non-sponsored content was diverse. It focused both on gaining new information and strengthening emotional bonds. Viewers also commended influencers for their expertise and investment. This was most prevalent across all three influencers' channels in their Halloween tutorials. Viewer comments on these videos were the most positive of any category, commending influencers for their investment and artistic abilities, with comments such as "You outdid yourself with this tutorial [Natalie]. Hands down the best Halloween look you've done to date" and "Damn editing skills on point." In non-sponsored content, viewers provided feedback in the form of assessing information gains and expressing personal opinions. The viewers projected their comments to both gain new information from viewers, as well as offer emotional resources for their investment and involvement.

Affiliate content and viewer feedback. In the affiliate/free product videos, there was still a focus on information gain. All fifteen videos in this section featured some type of product review or demonstration, so information gain was still a prominent aspect of the comments. There also was a larger proportion of response-based comments in affiliate/free product videos. Madden et al., (2012) suggested this category featured responses to previous comments and also can apply to the content creator as viewers respond to influencer prompts for engagement. One instance of this were two giveaways which occurred in affiliate content, where Caitlin and Danielle offered viewers the chance to win a product based on their response to a prompt provided in the video. While this was a more explicit example, response-based comments also were generated with subtler prompts throughout affiliate videos, such as when one viewer told Natalie, "I legit use to put bronzer on my whole face too.... like all over. ... lol love ya gurl." Viewers provided both direct and indirect responses based on influencer statements, as a way of indirectly conversing with the influencer. 
Affiliate content also produced a high ratio of comments described as heavily opinion-

based in Madden et al.'s (2012) framework. Madden et al. (2012) define opinion comments as,

... those in which commenters request or give their points-of-view on a video, person, object or topic. They differ from Advice comments because they are not encouraging actions, and are unlike Information comments because they contain explicit and subjective value judgements. They can be distinguished from Impression comments because they are longer, are more likely to be complete sentences, and are more likely to contain some form of evidence or justification for the perspective offered by the commenter (p. 706).

Opinion comments are unique in they express personal judgments more defined in terms of context. They are not initial reactions but those which have taken time to formulate and often address an argument the viewer may have toward the content. Most of these comments would be classified as the subcategory give (mixed) opinions (Madden et al., 2012) in which the comments addressed a negative concern followed by a positive appraisal, or vice-versa. These comments frequently included a buffer statement, in which viewers would provide their feedback and then lessen its impact by stating, "This isn't a hate comment" or "not trying to sound rude at all." Viewers appeared as if they wanted to provide feedback to influencers but were almost fearful their disagreement or negativity would impact them personally in the community. They structured their comments in a way which provided praise to the influencer, to create a more genuine tone to the comment, then added their negative opinions.

In four affiliate videos viewers directly addressed concerns they had with influencer content both in the information provided and emotional connections. In an affiliate video by Danielle viewers suggested her choice to use eyeliner markers in a coloring book was not fun and cute but rather detracted from the nature of the video. One viewer suggested, "I did not learn anything about the product - nor did I see an actual demo....maybe consider changing the title?" However, in keeping with the tone of affiliate videos, she finished her commenting by noting it 
was "just feedback," suggesting it was not meant in a hurtful tone but in a way to help Danielle place her content more appropriately. Beyond feedback on informational content, viewers also provided comments in regard to emotional resources. One viewer suggested Natalie was "just forcing everything" and her videos were less genuine. Another noted, "call me crazy but you just seem off, like you don't really wanna 'be here' or whatever.' In similar fashion, while these comments addressed direct concerns over Natalie's emotional connection, they still carried the buffer statements to lessen the impact. The first commenter noted,

I love you always and forever which is probably why most of us notice a difference in you... your happiness is all we care about so hey if this is the real you now then of course we will all just get with the program and still support no matter what.

The second commenter offered a similar sentiment, buffering her question of Natalie's emotional commitment with

This isn't a hate comment just honestly concerned because you haven't seemed happy in any of your videos in months really. Wish you would do like a sit down real talk kinda video to let your supporters know what's been up with you because we see it.. maybe I'm wrong but hey.. hope that you're ok tho

Burke, Marlow, \& Lento (2009) found similar responses to social network posts, in which friends presented their negative comments with an empathetic tone, to lessen the negativity of the feedback. In affiliate content, viewers still perceived influencers as being more genuine, their credibility was rated higher than sponsored content and they engaged in more reciprocal actions. They were not trying to sell products to consumers, simply engaging with free goods provided to them through their status. The feedback by viewers echoed this.

Viewers were compelled to tell influencers when they noticed a discrepancy in their information or emotional connections, yet buffered it with empathy to suggest they truly care about the influencer. The viewers wanted influencers to be involved in the community as 
suggested in the comments on Danielle's channel and attempt to reach viewers through their feedback and opinions. This provided influencers the opportunity to make changes in time to help with better overall reception of their message.

Sponsored content and viewer feedback. As found in social capital, authenticity, and branding elements, sponsored content produced the most varied and direct responses by viewers across any of the sponsorship categories. Sponsored posts may lower influencer credibility, bring questions of reciprocity, skew authenticity, and elicit negativity toward brands when relationships appear superficial. Across the fifteen videos reviewed in the sponsorship category, ten included at least one top comment which directly addressed negative concerns with the partnership. Additionally, while some buffering of comments with empathetic statements did exist, viewers were much more direct in their negative feedback in sponsored content.

Based on Madden et al.'s (2012) framework, these comments are still opinion-based. They are not initial impressions but are formulated with justification for the feedback. However, in comparison to affiliate/free product videos, these comments are strongly negative and focus on aspects of disconnect in content and emotions. One viewer to Danielle's channel suggested her content appeared contrived and did not fulfill expectations, stating,

As someone who struggles with crazy curly hair. I don't appreciate this video. I mean maybe this is the look you want to achieve. but honestly I feel like this video was a little 'staged'. I just hope that you really meant what you said about these products, because if you didn't it's a shame. I've tried these on my hair and they did nothing but flatten my curls and made my hair heavy.

This viewer offered justification for her disapproval of the content, suggesting her personal experience led her to believe the content was created for monetary reasons, not to provide helpful information to viewers. There was no buffer statement added to this comment. The viewer did not apologize for her opinions, instead she offered counter advice to Danielle, 
suggesting a different hair care brand in hopes it would "help girls who struggled with the products she talked about in this video as well."

Beyond questions regarding the communication of information presented in these videos, viewers also elicited strong responses toward influencer emotions and commitment to the community. One viewer asked Natalie, “you barely upload anymore I wouldn't be surprised if you start losing your followers what's going on?" Another suggested Danielle's investment in her channel was lacking in sponsored content, stating, these videos "sometimes feels like watching a commercial channel." These comments were different from affiliate/free product videos in they directly addressed the notion of sponsored content and how it appeared to be less focused on the community and more toward monetary gains. The feedback provided by viewers directly addressed these concerns, and related them back to concepts of influencer authenticity and investment.

Viewer feedback and impact on beauty influencers. In conjunction with elements of social capital, authenticity, and branding, viewer comments also played a key role in the communicative practices of influencers. The types of comments provided by viewers had similar characteristics: they are based around information gain, initial impressions, or are opinions of the influencers content and availability of emotional resources. Across the three sponsorship types, viewer comments provided key feedback for influencers, which was reflected in their later content.

Influencers may not have directly addressed each comment but their actions suggested viewer feedback did influence their content. When one viewer inquired of Caitlin, "I have been noticing that your 'panda eyes' as you like to say is not as dark anymore. So I wanted to know if you are using anything different!" Caitlin responded three videos later by showing viewers how 
to cover her dark under eye circles with color correcting products. When another viewer requested of Natalie, "I would like to see a drug store haul... and more drugstore makeup tutorials.....," she responded by posting a drugstore Valentine's Day make-up tutorial three weeks later with "lots of new drugstore makeup" in the title. Caitlin and Danielle also engaged in answering viewer questions in the comment section, providing direct answers to inquiries. One viewer asked Caitlin, "how do makeup artists do the background theme like the color and stuff do you put up a wallpaper every now and then or what and where did you film this the new studio or the old?" to which she replied, "I use large sheets of colored paper and hang them in the background on a stand :)" Another viewer asked for a drugstore holiday make-up look for those on a budget, to which Caitlin replied in the comments, "Such a good idea love! Thank you:)" She then uploaded the tutorial a few weeks later. There were several instances in which viewer feedback regarding information gain was addressed by influencers either directly in the comments or in future videos, suggesting influencers do hear or read viewer feedback on their content and seek to produce videos relevant to viewer needs.

Beyond information gain, influencers also engaged viewer feedback in their impression management techniques. When a viewer question Danielle's use of the phrase "hey guys" in her video, suggesting it was non-inclusive of everyone in the community, Danielle provided her opinion to viewers and asked them to give her feedback on what she should do. Viewers suggested the introduction should remain the same noting, "Hey [Danielle]! yeah i don't believe it's offensive at all. and there are girls and guys watch in your channel..." while another suggested justification for the statements, saying, "I'm from Jersey and "guys" is a casual way of including everyone! I start every video say "hi guys". I think if you said "hey girls" you're excluding the guys watching!" Danielle thanked viewers in a later video for their continued 
support and acknowledged their impact, noting their feedback helped her to make important decisions regarding her channel.

The most prominent form of viewer feedback influencing content occurred through their sponsored content, in which influencers were directly faced with justifying their partnerships to viewers. An example of this was a sponsored video by a drugstore brand, which garnered more negative attention as viewers questioned Caitlin's judgment in not directly stating it was a sponsored post in the video content. Though Caitlin included an FTC disclaimer in the description box, she did not directly state the content was sponsored in the video itself. Viewers questioned whether Caitlin was trying to hide the sponsorship, based on viewer perception that the brand sponsoring the video produced lower quality products. Following the video's posting, Caitlin explained in a YouTube comment why she accepted the sponsorship, noting she enjoyed the drugstore makeup brand and has been using their products since high school. She then told viewers, "After this video I have two more [brand] sponsored videos which I will be sure to put in the title so that you all are aware right away." The two additional sponsored videos were posted during the time of this research and received higher like/dislike ratios than this initial content. Viewers appreciated Caitlin's honesty and engagement with them, through discussing the issue with her viewers, not to her viewers, and making actionable promises for future content. This showed viewers Caitlin was dedicated to the community and to producing content which was appealing to them. Caitlin went beyond providing viewers with simple information-gain and engaged with them in genuine discussion, addressing their concerns and noting their involvement in her life, saying "I love you guys and hope you all enjoy the videos I create! If anything you guys are more like my friends so I hope you guys can understand."

Feedback also impacted video content in more indirect fashion through the uploading 
schedule of influencers. All three influencers engaged in an upload schedule that limited sponsored video post uploads to no more than three in a row. Of the 41 sponsored videos posted across all three influencers, there were only nine instances when two or more sponsored videos were uploaded sequentially. Within those nine instances, there was an average of 9.33 days between each sponsored video upload. Influencers engaged in a cycle of uploading sponsored videos in between content heavily focused on non-sponsored or affiliate/free product content.

A possible explanation for this uploading schedule could be the feedback provided by viewers in sponsored video posts. Influencer authenticity was most often questioned in response to sponsored videos, where viewers suggested content appeared contrived and focused on monetary gains for the influencer, rather than the betterment of the community. In contrast, nonsponsored and affiliate videos were considered more light-hearted and focused on relationship maintenance through genuine communication with viewers.

To lessen the impact of sponsored video posts in the community, influencers chose to place them into rotating schedules, alternating between non-sponsored, affiliate, and sponsored content on a frequent basis. By doing so, they provided varying types of social capital, authenticity projections, and product placement. This strategy proved successful, as the average ratio increase between a sponsored post and the next video posted (either non-sponsored or affiliate/free product) was 15.08 likes to dislikes. In some instances, ratios significantly increased between videos. The most prevalent example of this was when Natalie posted a sponsored post, which received a 14.36 like/dislike ratio, and then posted a non-sponsored Halloween tutorial a week later which received a 154.90 ratio, producing an increase of 140.55 likes to dislikes. If viewers assumed their feedback was heard by influencers, these findings suggested they were more willing to engage with future content and support it, through both direct and indirect 
means.

These results suggested there may be a positive feedback loop in the YouTube beauty community. Positive feedback is defined by an increase or decrease in one variable causing the same type of change (increase or decrease) in a second variable (Aknin, Dunn, \& Norton, 2012). Burke et al. (2010) suggested the presence of a positive feedback loop in social network site activities, which this research also supports. (insert a specific example from your study to illustrate)

In this feedback loop, influencers projected content to benefits their needs, as well as those of the community as a whole. They created content which offered them monetary gains and established notoriety in the community in return for viewers' support of their channel and information gain. Viewer feedback closed the loop, as this research suggested viewers can influence the type of content influencers create (see Figure 11).

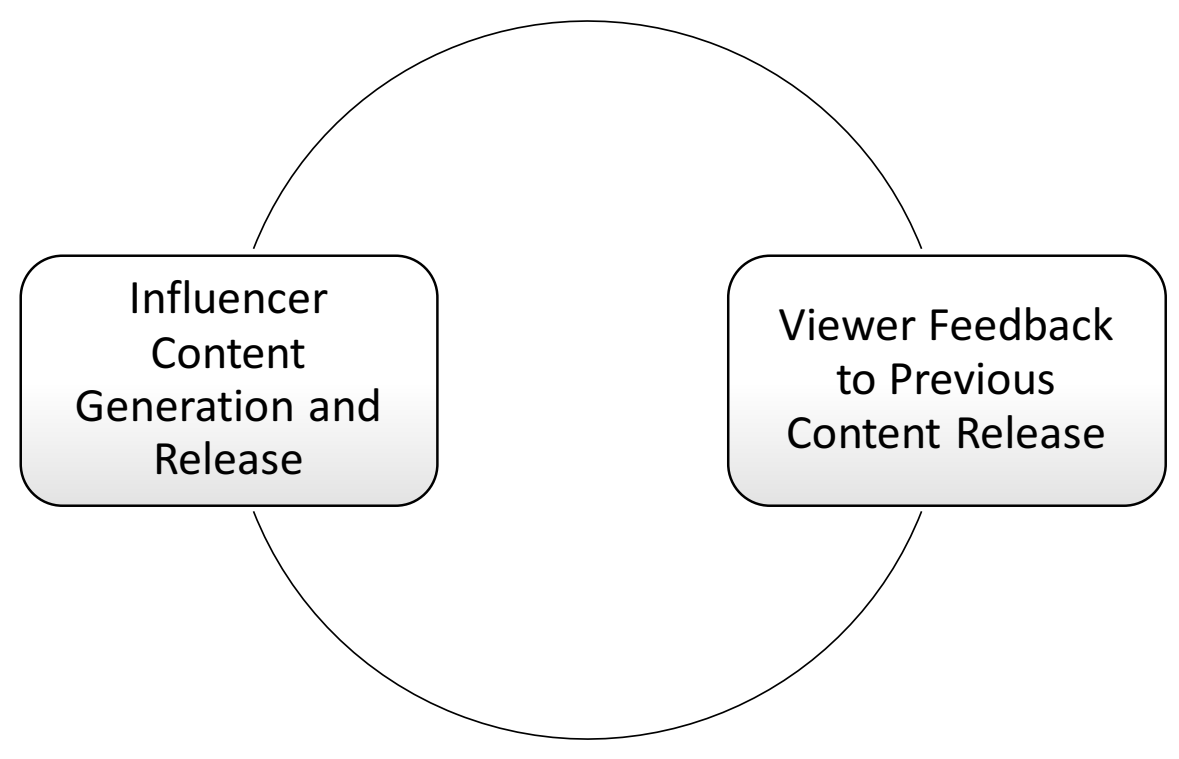

Figure 11. Positive feedback loop generated by viewer-influencer communication practices in YouTube beauty community 
This research showed viewers have a direct and indirect effect on the content released by influencers through their feedback. In non-sponsored and affiliate/free product videos, the majority of viewer feedback was positive, focused on information gain, impressions, and general opinions of the influencer's content. Influencers responded to this feedback by creating content suggested by viewers or providing them with further information gain opportunities. As this positive feedback from viewers rises, influencers appeared to grow more comfortable in their role in the community, eventually building to the release of a sponsored video based on the strength of their perceived relationships with viewers. If that sponsored post received positive feedback, they would release a second sponsored video, as there was no major impact in the loop from the first sponsored video. However, if viewers began to respond with negativity about the content or questioned the influencer's investment to the community, they then would release a non-sponsored or affiliate video. This action suggested indirectly that they hear viewers' feedback and want to provide them with meaningful content they desire, also bringing the feedback loop back to a stable position. In $70 \%$ of the instances in which influencers engaged in this action, they returned their views and like/dislike ratios to higher levels than in the previously sponsored video. While further research is needed to test the presence of this feedback loop in the YouTube beauty community over time, this research suggests increases in positive or negative feedback by viewers has a direct effect on the content released by influencers.

The features offered through the comments section on YouTube allowed beauty community members to engage in direct and indirect communication with beauty influencers. Viewers can impact influencer content through their comments, in addition to engaging with influencers in direct conversations. All three influencers demonstrated a tendency to rely on viewer feedback to guide their content, both in regard to creation and release. The feedback 
provided by viewers was beneficial to influencers, as it helped to establish their reliability and commitment to viewers, while also allowing them to receive personal gains through the careful placement of sponsored content.

\section{Influencer Monetization and Impact in the Digital Beauty Community}

This research served as an exploration of how social processes in a digital community were affected by personal gains, specifically financial advancement. The literature review revealed a lack of understanding in how the monetization of digital content was managed by influencers in regard to social structures in the digital environment, in addition to how it was received by viewers in the digital community. This research revealed the maintenance of social capital, projection of authenticity, branding principles, and communicative practices of digital beauty influencers were affected by personal factors of the influencer and the community as a whole.

Findings have shown digital influencers are able to tap into niche markets based on their relatability and success in the community (Tolson, 2010). These influencers are seen as being influential within the community, with a heightened presence, constructed through their impact online (Booth \& Matic, 2011; Senft, 2008). This presence has been granted through their identity as influencer, as an individual who is truly part of a digital community as an insider, rather than placed there for advertising purposes (Tolson, 2010).

This measure was complicated when influencers began to utilize their standing in the community to generate revenue for their content. YouTube is a unique social network site in it was one of the first to offer monetization for content, in addition to opening the door for influencers to gain additional revenue through product sponsorships (Freeman \& Chapman, 2007; YouTube, 2016a). The popularity of influencers on YouTube often was linked back to the 
impact they had on the community and relationships they had developed with viewers (Tolson, 2010). They were able to gain a reputation with their viewers, developed relationships among them, and utilized the bond to grow the community. This was achieved through the maintenance of social capital in the digital environment, which in turn was impacted by the perceived authenticity of the influencer. However, these constructs had not been explored in terms of how monetization of YouTube content can affect their impact in a digital community.

This research revealed digital influencers are able to maintain social capital, authenticity, branding measures, and communication within the community through their content but they should be mindful of viewer impact on these measures. Engagement in the digital culture was assessed by the viewer, through the criteria presented by the influencer. Viewers appear more willing to accept an influencer as being a leader when they are true to themselves and with the community, doing what they promised to. This helped to foster relationship growth, moving from the bridging capital of information gain to the bonding capital in emotional resources. Even when influencers engaged in branding practices for external beauty brands or modified their communicative practices, the notion of extending a sincere, genuine identity still produces favorable results for the influencers. It was when deviations occurred that viewers began to question their involvement in the community. As influencers continued to engage in creating content that was monetized for personal gains, viewers appeared willing to accept this if the influencer was upfront and genuine about her role in the community. 


\section{CHAPTER V: CONCLUSIONS}

The purpose of this research was to explore how beauty influencers crafted and maintained social capital and authenticity in conjunction with monetization activities, as a means of assessing the impact commoditization of content may have on digital influence and feedback within the community. This study addressed the main research question of: How do YouTube beauty influencers maintain social capital and project authenticity while engaging in monetization activities?

\section{Findings and Overall Implications of Research}

This research utilized an ethnographic content analysis to explore how the creation and maintenance of authenticity and social capital by digital YouTube beauty influencers were affected by monetization of content. It also assessed how these practices were affected by nonsponsored, affiliate/free content, and sponsored postings, as well as the communicative practices between influencers and viewers. This concluding chapter presents the findings in relation to how they answered the proposed research questions. It is to be noted sub-question four, addressing how beauty community viewers assess the constructs in this study, are discussed in each section respective to viewer impact on said category, as they serve as a form of assessing influencer projections of each construct by community members. Major contributions, limitations, potential for future research, and researcher reflections also are discussed.

Maintaining social capital. The first sub-question posed by this research asked: What practices are employed by YouTube beauty influencers to construct and maintain perceived social capital? The findings of this research suggested YouTube beauty influencers engaged in five practices to build trust and reciprocity, toward maintaining social capital in the digital community. 
Similarity, credibility, and impact worked together to create the first form of social capital demonstrated by YouTube beauty influencers: trust. When influencers possessed actual similarities to the influencers, they were provided with information gains both conceptual and real. Perceived similarities provided more emotional resources to the influencer and viewer, as it connected them based on abstract concepts of value systems, beliefs, and personalities. These findings were consistent with previous research on the power of relational, bonding social capital to modify individuals' actions, attitudes, and beliefs (Montoya et al., 2008). Thus, the presence of perceived similarities helped to generate bonding social capital in the system, as viewers and influencers were more willing to share personal details and philosophies, with the knowledge the community understood and valued their beliefs.

Credibility and impact provided an abstract and actionable construct to the exchange of resources. When influencers' information appeared to be honest and reliable, this research suggests viewers were more apt to respond to the content as credible. However, credibility established in this type of content does not appear to translate to sponsored posts. Across all three influencers, there were deviations among sponsored videos in the actual content and emotional demonstrations, affecting both bridging and bonding social capital negatively. This suggests while credibility did not require tangible proof of the trustworthiness of statements, viewers tended to perceive credibility as being higher when influencers produced content which appeared honest and sincere. These findings are in harmony with research suggesting credibility is established through generating trust that individuals demonstrate the true expertise and resources necessary to match their promises (Cullen et al., 2000; Kouzes \& Posner, 2011). In contrast, impact statements provided viewers with actionable advice, as a way to ground their opinions. Across all three sponsorship categories, influencers utilized impact statements as a way 
to provide advice to viewers. These impact statements served as a type of actionable trust element, providing advice in a physical form, rather than the abstract nature of credibility.

The second form of social capital displayed by the influencers was reciprocity. Involvement functioned as an indirect element of reciprocity, where influencers were able to utilize their engagement to the community as a way to receive resources from community members. The direct reciprocity demonstrated in bridging social capital was a rather straight forward process, where quantity defined the reciprocal actions. In terms of bonding social capital, involvement was situated around measures of quality. Viewers looked for influencers to be involved emotionally with their needs, providing content more personal to the community and focused on acts of support, such as providing encouragement to each other. These findings are in line with previous research suggesting involvement was related to directed communication and homogenization of digital communities (Burke et al., 2011; Valenzuela et al., 2009).

Investment demonstrated the actionable element of reciprocity. The investment of a beauty influencer was assessed through time and energy. Influencers were required to demonstrate a certain level of investment to their channel to reciprocate actions by viewers. There was an expectation that the influencer would share information and emotional resources with viewers, through the time and energy she dedicated to creating videos. If influencers provided reciprocity in a manner deemed fair and equal by viewers, these findings suggested viewers would engage in reciprocal actions, such as subscribing to the channel or liking videos, thereby increasing social capital, and the potential for monetization, to the influencer.

Authenticity - The fake-real beauty influencer. The second sub-question posed by this research asked: What practices are employed by YouTube beauty influencers to construct and maintain perceived authenticity? While beauty influencers self-identified as being real-real, this 
research suggested they should re-categorize themselves as fake-real, based on deviations in their projected authenticity.

Based on these results, beauty influencers should adopt a fake-real authentic identity in order to remain true to themselves and their content. Influencers should be upfront about the ways they define information on their channels and remain true to those definitions. If an influencer wants to create glamorous make-up tutorials every day, then relaying this information to viewers and defining her reasons why will help her to appear more authentic overall. Additionally, influencers should be sincere about the content they generate. If they are paid to sponsor a product, they should be explicit about this partnership and only recommend products they truly stand behind. Viewers understand the need for influencers to monetize their content. The greatest questions of authenticity did not arise out of whether influencers should engage in partnerships but rather questioned the sincere, genuine nature of this content. If influencers make their partnerships known in advance and choose brands which align with their identities, then their sponsored content will appear more authentic and sincere to viewers.

As Gilmore and Pine (2007) suggested, aligning oneself with the fake-real authenticity category did not mean an influencer was fake in a negative sense. Rather it acknowledged the self-motivated tendencies that are human nature for most individuals and helps to generate a sincerity in the content being provided. Being fake-real acknowledges that content is not being created out of pure selflessness but does have a purpose and connection to the influencer, which actually appears to be required of the viewers. Viewers do not wish to view content that is misplaced within the community. These viewers watch beauty videos to gain information and emotional resources through the influencer and the community which has been created. Therefore, to be true to the community, the influencer should be true to herself and recognize 
that there are self-motivated tendencies in this type of content. By recognizing this and being transparent about her authenticity offerings, the influencer is able to place herself as relevant to the community.

This research also provided support for the real-fake matrix to be applied to the personas-brand individual, in an effort to create and maintain a brand identity. As influencers function as their own personal brands, they must assess themselves as such, projecting an authenticity which was transferrable across a variety of target audiences. This research suggests the proper identification of an authenticity category can function in a practical setting based on the influencer's status as a person and a brand.

Impact on brand identity by influencers. The third sub-question in this research addressed: How do YouTube beauty community viewers respond to monetization activities through the comments section offered by YouTube? Aaker's (1996) model suggests a brand identity should carry a brand as person element. When the brand functions as a person, it is able to impart personality traits in an effort to humanize the brand, while also engaging in relationships with consumers. While this attempt helps to provide a human-like image to the brand, this research suggests beauty brands could utilize influencers to provide an actual human image to their products. It is integral to note, though, this research recognized the impact that the fluctuations in human personalities and images could have on a brand. Therefore, it is suggested this relationship occur organically, through a cyclical process (see Figure 10, p. 181), which recognizes the affordances of partnering with an influencer to maintain a brand identity, not build it.

If brands have built a noticeable connection to the influencer, through the cyclical nature of non-sponsored, affiliate/free product, and sponsored content, then the relationship was seen by 
viewers as being honest and lasting. If influencers mentioned a product a single time in a sponsored video and then never featured it again, these findings suggested viewers were quick to question whether these products truly do have a place in influencers' lives or whether they are suggested based solely on the monetization the influencer was receiving. In contrast, if an influencer built a reputation for engaging with the brand's products in the past, over an appropriate length of time and consistent interaction, these results showed viewers were more likely to commend influencers for their opportunity to work with the brand through sponsorships, as the relationship between the influencer and brand was seen as being genuine.

Through this relationship, influencers are able to provide actual human personality traits to the brand, connecting a face to the product. As the brand has purposefully chosen these influencers, it was assumed they feel these individuals align with their chosen brand identity, providing similar personality traits and communicative factors as pre-defined by the brand. Therefore, if the brand is able to develop a lasting relationship with the influencer, it can benefit from maintaining its brand identity through the person-as-brand.

Message reception and positive feedback loop. In addition to elements of social capital, authenticity, and branding, the communicative abilities of YouTube also allowed for an assessment of sender-receiver message functions in the digital beauty community. This research proposed there is the potential for a positive feedback loop in the YouTube beauty community, where viewer feedback informs influencer content, which then informs viewers and generates additional feedback (see Fig.11, p. 209).

In this feedback loop, influencers projected content which benefitted their needs, as well as those of the community as a whole. They are creating content that offered monetary gains and established notoriety in the community, in return for viewers' support of their channel and 
information gain. Viewer feedback closed the loop, as this research suggested viewers were able to influence the type of content influencers create. Content was directly impacted by viewer feedback in regard to influencers' creating videos which addressed viewers needs or requests for further information. Content also was impacted in more indirect means, as apparent when assessing the differences in feedback between non-sponsored, affiliate/free product, and sponsored content. While further research is needed to verify the existence of this across the beauty community as a whole, this research suggested the possibility for the presence of a positive feedback loop in these three influencers' communities.

\section{Opportunities for Future Research and Constraints of Study}

This research was a targeted look at how YouTube beauty influencers engage in maintaining both authenticity and social capital in conjunction with monetization of content, as a means of assessing the impact commoditization of content may have on digital influence. This research also assessed the specific branding and communicative practices influencers engaged in while monetizing their content, as a way to determine how influencers extend their reach beyond their personal channels and communities.

From these results, several opportunities for building upon this research have emerged.

First, these beauty influencers were assessed over a one-year time period, during which time the parameters of the research required them to upload a specific percentage of beauty-related content. While this parameter purposefully was chosen to explore how the areas of interest to this research function in a community by established influencers who engage in a variety of monetization activities, assessing the growth of each influencer's channel over the duration of its existence could provide further evidence of how social capital and authenticity are built, as well as their changes over time. By addressing the influencers from the beginning of their channels 
inception to its current state, future research could produce trajectories of how each of the measures in this study were built and how they changed with monetization efforts. This would provide further evidence to the cycles and processes established in this research.

Additionally, while this study chose to focus on YouTube as it was one of the first social network sites to offer monetization opportunities and thus attracted influencers to the site in its early stages, this research could be expanded to address monetization on other social network platforms. For example, Instagram and SnapChat now offer opportunities for sponsored posts by influencers. The static image nature of Instagram and the quick time to view of SnapChat each provide unique opportunities to understand how influencers utilize these sites to grow their communities and gain revenue. Future research also can look at how YouTube influencers might expand their digital communities onto these other social network sites in an effort to monetize different types of content and attract viewers with a variety of content offerings.

As this research was structured to be an initial appraisal of influencer projections as may be assessed by viewers, with myself functioning as an insider member of the digital community, it did not allow for the opportunity to engage the influencers in direct conversation. Future research should aim to connect directly with influencers to address their perceptions of social capital maintenance and to help define what authenticity means to them in a digital context. In addition, this research could not fully address the potential for a positive feedback loop, as constraints did not allow for the tracking of viewer comments over time nor did it permit engaging directly with the viewers. This research could be expanded through interacting with viewers to assess their motivations for communicating with the influencer over time and perceptions of influencer engagement in the community. This would provide additional support for the presence of a positive feedback loop in this digital community. 
Finally, this study focused exclusively on beauty influencers in the YouTube beauty community. While the beauty community is one of the most expansive networks on YouTube, the role influencers play in other communities should be assessed to better understand if monetization functions similarly among YouTube influencers as a whole. By assessing other genres of YouTube videos, it would help to determine whether monetization has any similarities across content, suggesting it plays an integral role in influencer-viewer relationships regardless of content type. This would provide a level of practical knowledge to influencers, as well as branding knowledge to companies wishing to partner with them, in understanding how monetization was received and impacts the community within which they craft their content. This would help to determine the sustainability of influencers, both for their personal careers as well as for consumer-generated advertising affordances.

\section{Researcher Reflections}

The major findings in this research suggested influencers were able to maintain social capital, authenticity, branding measures, and communication with viewers, even in the presence of monetization. However, this research also proposed each of these tasks could become more burdensome for the influencer when engaging in sponsorships with brands or creating content deemed emotionally devoid by viewers. Of interesting note was that these features appeared to function across continuums or within a cycle. For instance, social capital and authenticity could be immediately impacted by new content creation. Viewers were quick to judge content on a video-level basis, where the appraisal of social capital or authenticity could quickly shift based on the perceived sincerity, reciprocity, and genuine nature of the influencer. Branding and communication function on a more cyclical nature, with influencers and viewers engaging in 
multiple tradeoffs, until an acceptable end goal was reached for both parties, thus restarting or redefining the cycles.

It is important to assess this research for any potential biases which could have influenced qualitative research. A digital ethnographic content analysis was chosen for this research as it allowed the research to take into account the nature of the digital social environment and the affordances of the platform upon which this culture exists (Rogers, 2013). This allowed the richness of the experiences of influencers and the community within which they took place to create an understanding of shared practices between viewers and influencers. As such, the research questions were phrased in a way that accounted for both perspectives to be explored. The intent of this digital ethnography was to present the mechanisms by which influencers project concepts of social capital, authenticity, branding, and communication, in addition to how viewers assess them, in an effort to create shared understanding on the concepts.

In an attempt to understand and define these practices, the biases that lie with myself as the researcher also must be addressed. I identify as a member of the beauty community on YouTube, however, I do not create content on the platform. Therefore, I am not an influencer but am a viewer of the content. It was vital my review of these constructs not be skewed by my position but rather serve as an interpretation from an inside perspective on the practices influencers engage with in this community. As a participant in this community, I want influencers to continue to provide the informational and emotional resources to viewers which have garnered them their standing. However, I also want viewers to understand influencer content was directly impacted by monetization, which was a necessity for them to keep their channels updated with current products and develop videos which are entertaining, as well as informative. Influencers must generate some form of revenue to sustain their channels, as they 
utilize YouTube as a source of income, and the time investment they dedicate to these videos should mirror such commitment. As an academic studying the impact of digital culture, I want to help others to understand the affordances of digital communities and to question how these cultures can impact social structures, both on- and offline.

While this research addressed the questions that were asked, it also brings about much larger questions of digital culture as a whole. For instance, how are influencers affected by their roles in these communities? Do they feel any personal strain in work-life balance when their lives essentially become their work? How can the emotional labor embedded in being "on camera" nearly 24/7 impact their social structures offline? Additionally, this research offers additional questions of consumption of this content. In what ways do the projections of influencer identity affect the self-constructs of viewers? How do viewers separate the perceived from the reality, in regard to the asynchronous nature of video content versus the more synchronous communications of daily life? These questions provide a more philosophical view of this research, which likely would be assessed differently based on the researcher and the methods upon which the questions were addressed. This illustrates the amorphous nature of this type of research and the need to address any potential biases when engaging in qualitative studies.

This research discovered there are mechanisms by which influencers can monetize content and still engage with the digital communities in which they function. However, it also discovered the relationships between influencers and viewers are widespread, with different viewers addressing different needs. The influencer must navigate this community in a way to present a cohesive identity which was transferable across diverse groups of individuals. As influencers continue to engage in monetization practices, it is essential they remember where 
their notoriety came from. They were offered these affordances based on being relatable human beings, not carefully calculated brands. While their new roles may necessitate being cautious with their identity, they should retain the humanity that won their viewers over from the beginning. 


\section{APPENDIX A OPERATIONAL DEFINITIONS}

Digital influencers: Individuals on the Internet who have gained a large following of viewers and heightened digital presence through digital means (Tolson, 2010)

Beauty influencers: Individuals who have generated a notable digital presence through their engagement in beauty-related content generation and are able to utilize their impact to position themselves as relevant in the beauty community

YouTube beauty community: The digital culture on the YouTube social network platform within members seek out and engage with beauty-related content toward building and maintaining viable communities of interest

Beauty community viewers: Individuals who view beauty influencer video content and engage in active or passive consumption.

Social capital: The acquisition of shared resources through the interactions of a members in a network (Bourdieu, 1985)

Bridging social capital: The exchange of informational resources within a community in an effort to obtain unique content based on the diverse contributions of community members (Putnam, 2000)

Bonding social capital: The exchange of scarce emotional resources within a community; occurs through group homogenization when information gain becomes narrower based on group member similarity, however, the strengthening of relationships between members creates the opportunity for creating emotional bonds (Putnam, 2000)

Authenticity: The operation of one's true self in everyday life, as presented through genuine portrayals of identity constructs (Goldman \& Kernis, 2002)

Real authenticity constructs: Defined by being true to the self and being/doing what it says it is (Gilmore \& Pine, 2007)

Fake authenticity constructs: Defined by not being true to the self and not being/doing what it says it is (Gilmore \& Pine, 2007)

Brand identity: Promotion of the constructed reality of the company, as a way to tell the consumers who the brand is and what it stands for (Aaker, 1996; de Chernatony, 1999)

Positive feedback loop: An increase or decrease in one variable causes the same type of change (increase or decrease) in a second variable (Aknin, Dunn, \& Norton, 2012) 


\section{REFERENCES}

Aaker, D. A. (1991). Managing brand equity: Capitalizing on the value of a brand name. New York: The Free Press.

Aaker, D. A. (1992). Managing the most important assets: Brand equity. Planning Review, 20(5), 56-58.

Aaker, D. A. (1996). Building strong brands. New York: The Free Press.

Aaker, D. A. \& Joachimsthaler, E. (2000). Brand leadership: The next level of the brand revolution. New York: The Free Press.

Aaker, J. (1997). Dimensions of brand personality. Journal of Marketing Research, 34(3), 347356.

Aaker, J., Fournier, S., \& Brasel, S. A. (2004). When good brands do bad. Journal of Consumer Research, 31(1), 1- 16.

Abcarian, R. (2013, May 21). A plus-size protest to Abercrombie and Fitch's backward thinking. Retrieved from http://articles.latimes.com/2013/may/21/local/la-me-ln-protestabercrombie-and-fitch-20130521

Abidin, C., \& Ots, M. (2015). The Influencer's dilemma: The shaping of new brand professions between credibility and commerce. In AEJMC 2015, Annual Conference, San Francisco, CA, August 6-9.

Adam, H. \& Galinsky, A. D. (2012). Enclothed cognition. Journal of Experimental Social Psychology, 48(4), 918-925.

AdvertisingAge. (2003, September 15). Crest. Retrieved from http://adage.com/article/adageencyclopedia/crest/98424/

Aghaei, S., Nematbakhsh, M. A., \& Farsani, H. K. (2012). Evolution of the World Wide Web: From Web 1.0 to Web 4.0. International Journal of Web \& Semantic Technology, 3(1), $1-10$.

Aknin, L. B., Dunn, E. W., \& Norton, M. I. (2012). Happiness runs in a circular motion:

Evidence for a positive feedback loop between prosocial spending and happiness. Journal of Happiness Studies, 13(2), 347-355.

Alba, J. W., Lynch, J., Weitz, B., Janiszewski, C., Lutz, R., Sawyer, A., \& Wood, S. (1997). Interactive home shopping: Consumer, retailer, and manufacturer incentives to participate in electronic marketplaces. Journal of Marketing, 61(3), 38-53. 
Altheide, D. L. (1987). Reflections: Ethnographic content analysis. Qualitative sociology, 10(1), 65-77.

Alton, L. (2015, July 29). 7 ways to make your brand more authentic on social media. Retrieved from https://socialmediaweek.org/blog/2015/07/more-authentic-brands/

Ambler, T. (1992). Need-to-know marketing: An accessible A to Z guide. London: Century Business.

American Anthropological Association. (2016). American Anthropological Association Statement on Ethnography and Institutional Review Boards (Adopted by AAA Executive Board June 4, 2004). Retrieved from http://www.americananthro.org/ParticipateAnd Advocate/Content.aspx?ItemNumber $=1652$

Ardévol, E. \& Gómez-Cruz, E. (2014). Digital ethnography and media practices. In A.N. Valdivia \& F. Darling-Wolf (Ed. and Volume Ed.), The international encyclopedia of media studies: Research methods in media studies $\left(7^{\text {th }}\right.$ Ed.). Hoboken, NJ: John Wiley \& Sons, Ltd.

Archer, C., Pettigrew, S., \& Harrigan, P. (2014). A tale of power, passion and persuasion: Bloggers, public relations and ethics. Asia Pacific Public Relations Journal, 15(1), 37-54.

Arvidsson, A. (2013). The potential of consumer publics. Ephemera, 13(2), 367-91.

Ba, S., \& Pavlou, P. A. (2002). Evidence of the effect of trust building technology in electronic markets: Price premiums and buyer behavior. MIS quarterly, 243-268.

Baym, N. (2010). Personal connection in the digital age: Digital media and society series. Cambridge: Polity Press.

Bennett, S. (2013, April 29). 6 amazing social media statistics for brands and businesses [Infographic]. Retrieved from http://www.adweek.com/socialtimes/social-mediafacts/482462

Berg, B. L. (2008). Qualitative research methods for the social sciences ( $7^{\text {th }}$ Ed.). Boston: Allyn \& Bacon.

Billett, S. (1998). Situation, social systems and learning. Journal of Education and Work, 11(3), 255-274.

Blanchard, A., \& Horan, T. (1998). Virtual communities and social capital. Social science computer review, 16(3), 293-307.

Blomqvist, K. (1997). The many faces of trust. Scandinavian journal of management, 13(3), 271-286. 
Booth, N. \& Matic, J. A. (2011). Mapping and leveraging influencers in social media to shape corporate brand perceptions. Corporate Communications: An International Journal, 16(3), 184-191.

Bourdieu, P. (1985). The forms of capital. In J. G. Richardson (Ed.), Handbook of Theory and Research for the Sociology of Education (p. 241-258). New York: Greenwood

boyd, d. m., \& Ellison, N. B. (2007). Social network sites: Definition, history, and scholarship. Journal of Computer-Mediated Communication, 13(1), 210-230.

boyd, d. (2011). Social networking sites as networked publics: Affordances, dynamics and implication. In Z. Papacharissi (Ed.), A networked self: Identity community and culture on social network sites (p. 39-58). London: Routledge.

Briggs, P., Burford, B., De Angeli, A., \& Lynch, P. (2002). Trust in online advice. Social science computer review, 20(3), 321-332.

Brown, B. (2008). Bobbi Brown makeup manual: For everyone from beginner to pro. London: Headline Publishing Group.

Burke, M., Kraut, R., \& Marlow, C. (2011, May). Social capital on Facebook: Differentiating uses and users. In Proceedings of the SIGCHI conference on human factors in computing systems (pp. 571-580). ACM.

Burke, M., Marlow, C., \& Lento, T. (2009, April). Feed me: motivating newcomer contribution in social network sites. In Proceedings of the SIGCHI conference on human factors in computing systems (pp. 945-954). ACM.

Burmann, C., Jost-Benz, M., \& Riley, N. (2009). Toward an identity-based brand equity model. Journal of Business Research, 62(3), 390-397.

Burnard, P. (1992). A method of analyzing interview transcripts in qualitative research. Nurse Education Today, 11(6), 461-466.

Burns, K.S. (2016). How the top social media brands use influencer and brand advocacy campaigns to engage fans. In A. L. Hutchins \& N. T. J. Tindall (Eds.), Public relations and participatory culture: Fandom, social media, and community engagement (pp. 5870). New York: Routledge.

Calfee, J. E. \& Ringold, D. J. (1994). The 70\% majority: Enduring consumer beliefs about advertising. Journal of Public Policy and Marketing, 19(2), 228-38.

Caliandro, A. (2016). Ethnography in digital spaces: Ethnography of virtual worlds, netnography, and digital ethnography. In R. Denny \& P. Sunderland (Eds.), Handbook of anthropology in business (p. 658-680). New York: Routledge. 
Chatzopoulou, G., Sheng, C., \& Faloutsos, M. (2010, March). A first step toward understanding popularity in YouTube. In INFOCOM IEEE Conference on Computer Communications Workshops, 2010 (pp. 1-6). IEEE.

Chau, C. (2010). YouTube as a participatory culture. New Directions for Youth Development, 2010(128), 65-74.

Chiu, C. M., Hsu, M. H., \& Wang, E. T. (2006). Understanding knowledge sharing in virtual communities: An integration of social capital and social cognitive theories. Decision support systems, 42(3), 1872-1888.

Coleman, J. S. (1988). Social capital in the creation of human capital. American journal of sociology, 94, S95-S120.

Constant, D., Sproull, L., \& Kiesler, S. (1996). The kindness of strangers: The usefulness of electronic weak ties for technical advice. Organization science, 7(2), 119-135.

Constantinides, E., Lorenzo-Romero, C., \& Gomez-Boria, M. (2009). Social media: A new frontier for retailers? European Retail Research, 22, 1-28.

Coombe, R. J. (1993). The properties of culture and the politics of possessing identity: Native claims in the cultural appropriation controversy. The Canadian Journal of Law and Jurisprudence, 6(02), 249-285.

Cormode, G., \& Krishnamurty, B. (2008). Key differences between Web 1.0 and Web 2.0. First Monday, 13 (6). Retrieved from http://firstmonday.org/article/view/2125/1972

Cova, B. \& White, T. (2010). Counter-brand and alter-brand communities: The impact of Web 2.0 on tribal marketing approaches. Journal of Marketing Management, 26(3-4), 256270 .

Cowan, R. S. (1997). A social history of American technology. New York: Oxford University Press, Inc.

Creswell, J. W., \& Miller, D. L. (2000). Determining validity in qualitative inquiry. Theory into practice, 39(3), 124-130.

Cullen, J. B., Johnson, J. L., \& Sakano, T. (2000). Success through commitment and trust: The soft side of strategic alliance management. Journal of World Business, 35(3), 223-240.

de Chernatony, L. (1999). Brand management through narrowing the gap between brand identity and brand reputation. Journal of marketing management, 15(1-3), 157-179.

de Chernatony, L. \& Riley, F. D. (1997). Modelling the components of the brand. European Journal of Marketing, 32(11,12), 1074-1090. 
Denizet-Lewis, B. (2006, Jan. 24). The man behind Abercrombie \& Fitch. Retrieved from http://www.salon.com/2006/01/24/jeffries/

Denzin, N. K. \& Lincoln, Y. S. (Eds.). (2005). The sage handbook of qualitative research ( $3^{\text {rd }}$ Ed.). Thousand Oaks, CA: Sage Publications, Inc.

Dey, I. (1993). Qualitative data analysis: A user-friendly guide for social scientists. New York: Routledge.

Dolich, I. J. (1969). Congruence relationships between self images and product brands. Journal of Marketing Research, 6(1), 80-84.

Doney, P. M., \& Cannon, J. P. (1997). An examination of the nature of trust in buyer-seller relationships. the Journal of Marketing, 35-51.

Eisenberg, L. (2009). Shoptimism: Why the American consumer will keep on buying no matter what. New York: The Free Press.

Ellison, N. B., Steinfield, C., \& Lampe, C. (2007). The benefits of Facebook "friends:” Social capital and college students' use of online social network sites. Journal of ComputerMediated Communication, 12(4), 1143 - 1168.

Ellison, N. B., Steinfield, C., \& Lampe, C. (2011). Connection strategies: social capital implications of Facebook-enabled communication practices. New Media \& Society, 13(6), 873-892.

Elo, S. \& Kyngäs, H. (2007). The qualitative content analysis process. Journal of Advanced Nursing, 62(1), 107-115.

Federal Trade Commission. (2015, May). The FTC's endorsement guidelines: What people are asking. Federal Trade Commission. Retrieved from https://www.ftc.gov/tipsadvice/business-center/guidance/ftcs-endorsement-guides-what-people-are-asking

Fennis, B. M. \& Pruyn, A. (2007). You are what you wear: Brand personality influences on consumer impression formation. Journal of Business Research, 60(6), 634-639.

Fischer, C. S. (1992). America calling: A social history of the telephone to 1940. Berkeley and Los Angeles, CA: University of California Press.

Fournier, S., \& Avery, J. (2011). The uninvited brand. Business Horizons, 54(3), 193-207.

Freeman, B., \& Chapman, S. (2007). Is "YouTube" telling or selling you something? Tobacco content on the YouTube video-sharing website. Tobacco Control, 16(3), 207-210. 
Gale, N. K., Heath, G., Cameron, E., Rashid, S., \& Redwood, S. (2013). Using the framework method for the analysis of qualitative data in multi-disciplinary health research. $B M C$ Medical Research Methodology, 13(1), 117.

Garcia, T. (2011, February 3). Kenneth Cole's Twitter fail. Retrieved from http://www.adweek.com/prnewser/kenneth-coles-twitter-fail/15371?red=pr

Gardner, B. B., \& Levy, S. J. (1955). The product and the brand. Harvard Business Review, 33(2), 33-39.

Getting, B. (2007, April 18). Basic definitions: Web 1.0, Web 2.0, Web 3.0. Retrieved from http://www.practicalecommerce.com/articles/464-Basic-Definitions-Web-1-0-Web-2-0Web-3-0

Gilbreath, B. (2010). The next evolution of marketing: Connect with your customers by marketing with meaning. New York: McGraw-Hill.

Gilmore, J. H. \& Pine, B. J. (2007). Authenticity: What consumers really want. Boston: Harvard Business School Publishing.

Goffman, E. (1963). Behavior in public places: Notes on the social organization of gatherings. New York: The Free Press.

Goldman, B. M., \& Kernis, M. H. (2002). The role of authenticity in healthy psychological functioning and subjective well-being. Annals of the American Psychotherapy Association, 5(6), 18-20.

Granovetter, M. (1973). The strength of weak ties. American Journal of Sociology, 78(6), 13601380 .

Greenhow, C. \& Robelia, B. (2009). Old communication, new literacies: Social network sites as social learning resources. Journal of Computer-Mediated Communication, 14(4), 11301161.

Griffith, E. (2011). Bloggers mean business. Adweek, 9, 58-59.

Hammersley, M., \& Atkinson, P. (2007). Ethnography: Principles in practice. Routledge.

Hampton, K. N., Goulet, L. S., Marlow, C., \& Rainie, L. (2012). Why most Facebook users get more than they give. Pew Internet \& American Life Project, 3, 1-40.

Haridakis, P., \& Hanson, G. (2009). Social interaction and co-viewing with YouTube: Blending mass communication reception and social connection. Journal of Broadcasting \& Electronic Media, 53(2), 317-335. 
Harris, F., and de Chernatony, L. (2001). Corporate branding and corporate brand performance. European Journal of Marketing, 35(3/4), 441-456.

Henderson, A. \& Bowley, R. (2010). Authentic dialogue? The role of "friendship" in a social media recruitment campaign. Journal of Communication Management, 14(3), 237-257.

Herbig, P., \& Milewicz, J. (1993). The relationship of reputation and credibility to brand success. Journal of consumer marketing, 10(3), 18-24.

Hine, C. (2000). Virtual ethnography. London: Sage.

Hine, C. (2007). Connective ethnography for the exploration of e-Science. Journal of ComputerMediated Communication, 12(2), 284-300.

Holt, D. B. (1995). How consumers consume: A typology of consumption practices. Journal of Consumer Research, 22(1), 1-16.

Hong, J. W., Muderrisoglu, A., \& Zinkhan, G. M. (1987). Cultural differences and advertising expression: A comparative content analysis of Japanese and US magazine advertising. Journal of advertising, 16(1), 55-68.

Hong, J. W., \& Zinkhan, G. M. (1995). Self-concept and advertising effectiveness: The influence of congruency, conspicuousness, and response mode. Psychology \& Marketing, 12(1), 53-77.

Horton, D., \& Richard Wohl, R. (1956). Mass communication and para-social interaction: Observations on intimacy at a distance. Psychiatry, 19(3), 215-229.

Hsieh, H., \& Shannon, S. E. (2005). Three approaches to qualitative content analysis. Qualitative Health Research, 15(9), 1277-1288.

Igarashi, T., Kashima, Y., Kashima, E. S., Farsides, T., Kim, U., Strack, F., \& Yuki, M. (2008). Culture, trust, and social networks. Asian Journal of Social Psychology, 11(1), 88-101.

Instagram. (2015, September 22). Celebrating a community of 400 million. Retrieved from http://blog.instagram.com/post/129662501137/150922-400million

Jamal, A. \& Goode, M. M. H. (2001). Consumers and brands: A study of the impact of selfimage congruence on brand preference and satisfaction. Marketing Intelligence \& Planning, 19(7), 482-492.

Jenkins, H., Purushotma, R., Weigel, M., Clinton, K., \& Robison, A.J. (2006). Confronting the challenges of participatory culture: Media education for the $21^{\text {st }}$ century. Chicago, IL: The John D. and Catherine T. MacArthur Foundation. Retrieved from: http://mitpress.mit.edu/sites/default/files/titles/free_download/9780262513623_ Confronting_the_Challenges.pdf 
Johnston, J. \& Taylor, J. (2008). Feminist consumerism and fat activists: A comparative study of grassroots activism and the Dove Real Beauty campaign. Signs, 33(4), 941-966.

Kabadayi, S., \& Price, K. (2014). Consumer-brand engagement on Facebook: liking and commenting behaviors. Journal of Research in Interactive Marketing, 8(3), 203-223.

Kapferer, J. (1994). Strategic brand management: New approaches to creating and evaluating brand equity. New York: The Free Press.

Kapferer, J. (2008). The new strategic brand management: Creating and sustaining brand equity long term ( $4^{\text {th }}$ ed.). London: Kogan Page, Ltd.

Kaplan, A. M. \& Haenlein, M. (2010). Users of the world, unite! The challenges and opportunities of social media. Business Horizons, 53(1), 59-68.

Kavanaugh, A. L., Reese, D. D., Carroll, J. M., \& Rosson, M. B. (2005). Weak ties in networked communities. The Information Society, 21(2), 119-131.

Kietzmann, J. H., Hermkens, K., McCarthy, I. P., \& Silvestre, B. S. (2011). Social media? Get serious! Understanding the functional building blocks of social media. Business horizons, 54(3), 241-251.

Kondracki, N. L., Wellman, N. S., \& Amundson, D. R. (2002). Content analysis: Review of methods and their applications in nutrition education. Journal of Nutrition Education and Behavior, 34(4), 224-230.

Kotler, P. (1997). Marketing management: Analysis, planning, implementation, and control. Upper Saddle River, NJ: Prentice Hall.

Kouzes, J. M., \& Posner, B. Z. (2011). Credibility: How leaders gain and lose it, why people demand it (Vol. 244). John Wiley \& Sons.

Krampf, R., \& Ueltschy, L. (1997). The influence of acculturation on advertising effectiveness to the Hispanic market. Journal of Applied Business Research, 13(2), 87.

Kreber, C., Klampfleitner, M., McCune, V., Bayne, S., \& Knottenbelt, M. (2007). What do you mean by "authentic"? A comparative review of the literature on conceptions of authenticity in teaching. Adult Education Quarterly, 58(1), 22-43.

Kressmann, F., Sirgy, M. J., Herrmann, A., Huber, F., Huber, S., \& Lee, D. J. (2006). Direct and indirect effects of self-image congruence on brand loyalty. Journal of Business Research, 59(9), 955-964.

Krippendorff, K. (2013). Content analysis: An introduction to its methodology $\left(3^{\text {rd }} \mathrm{Ed}.\right)$. Thousand Oaks, CA: Sage Publications, Inc. 
Kumar, S., Massie, C., \& Dumonceaux, M. D. (2006). Comparative innovative business strategies of major players in cosmetic industry. Industrial Management \& Data Systems, $106(3), 285-306$.

Labrecque, L. I. (2014). Fostering consumer-brand relationships in social media environments: The role of parsocial interaction. Journal of Interactive Marketing, 28(2), 134-148.

Labrecque, L. I., Markos, E., \& Milne, G. R. (2011). Online personal branding: Processes, challenges, and implications. Journal of Interactive Marketing, 25(1), 37 - 50.

Lange, P. G. (2007). Publicly private and privately public: Social networking on YouTube. Journal of Computer-Mediated Communication, 13(1), 361-380.

Levy, M. R. (1979). Watching TV news as para-social interaction. Journal of Broadcasting \& Electronic Media, 23(1), 69-80.

Lincoln, Y. S., \& Guba, E. G. (1985). Naturalistic inquiry. London: Sage Publications, Inc.

Lipstein, E. A. \& Britto, M. T. (2015). The evolution of pediatric chronic disease treatment decisions: A qualitative, longitudinal view of parents' decision-making process. Medical Decision Making, 35(6), 703-713.

Livingstone, S. (2008). Taking risky opportunities in youthful content creation: Teenagers' use of social networking sites for intimacy, privacy, and self-expression. New Media \& Society, 10(3), 393-411.

Mangold, W. G., \& Faulds, D. J. (2009). Social media: The new hybrid element of the promotion mix. Business Horizons. 52(4), 357-365.

Mansor, N., Efni binti Mat Ali, D., \& Yaacob, M. R. (2010). Cosmetic usage in Malaysia: Understanding of the major determinants affecting the users. International Journal of Business and Social Science, 1(3), 273-281.

Marwick, A. (2013, July). 'They're Really Profound Women, They're Entrepreneurs': Conceptions of Authenticity in Fashion Blogging. In 7th International AIII Conference on Weblogs and Social Media (ICWSM), July (Vol. 8).

Marwick, A. E. (2013). Status update: Celebrity, publicity, and branding in the social media age. New Haven, CT: Yale University Press.

Marwick, A. E. \& boyd, d. (2011). I tweet honestly, I tweet passionately: Twitter users, context collapse, and the imagined audience. New Media \& Society, 13(1), 114-133.

Masten, D. \& Plowman, T. (2003). Digital ethnography: The next wave in understanding the consumer experience. Design Management Journal, 14(2), 75-81. 
Mayer, R. C., Davis, J. H., \& Schoorman, F. D. (1995). An integrative model of organizational trust. Academy of management review, 20(3), 709-734.

McGrath, C. A., Vance, C. M., \& Gray, E. R. (2003). With a little help from their friends: Exploring the advice networks of software entrepreneurs. Creativity and Innovation Management, 12(1), 2-10.

McKee, H.A. \& Porter, J. E. (2009). Playing a good game: Ethical issues in researching MMOGs and virtual worlds. International Journal of Internet Research Ethics, 2(1), 5-37.

Merskin, D. (2007). Truly toffee and raisin hell: A textual analysis of lipstick names. Sex Roles, 56(9), 591-600.

Miles, M. B. \& Huberman, A. M. (1994). Qualitative data analysis: An expanded sourcebook. Thousand Oaks, California: Sage.

Miller, M. (2011). YouTube for business: Online video marketing for any business. Indianapolis, IN: Que Publishing.

Montoya, R. M., Horton, R. S., \& Kirchner, J. (2008). Is actual similarity necessary for attraction? A meta-analysis of actual and perceived similarity. Journal of Social and Personal Relationships, 25(6), 889-922.

Morey, Y., Bengry-Howell, A., \& Griffin, C. (2012). Public profiles, private parties: Digital ethnography, ethics and research in the context of Web 2.0. In Innovations in youth research (pp. 195-209). Palgrave Macmillan UK.

Morris, M. \& Ogan, C. (1996). The Internet as mass medium. Journal of Computer-Mediated Communication, 1(4), n.p.

Mosendz, P. \& Bhasin, K. (2016, October 31). YouTube is helping to sell a lot of makeup. Retrieved from https://www.bloomberg.com/news/articles/2016-10-31/youtube-ishelping-to-sell-a-lot-of-makeup

Mourey, J. [jennamarbles]. (2016, July 21). Ultimate 100 coats of things video [Video file]. Retrieved from https://www.youtube.com/watch?v=hsFLMjlgR_o

Murthy, D. (2008). Digital ethnography: An examination of the use of new technologies for social research. Sociology, 42(5), 837-855.

Myers, C. A. (2003). Managing brand equity: A look at the impact of attributes. Journal of Product \& Brand Management, 12(1), 39-51.

Nahapiet, J., \& Ghoshal, S. (1998). Social capital, intellectual capital, and the organizational advantage. Academy of management review, 23(2), 242-266. 
Nahemow, L., \& Lawton, M. P. (1975). Similarity and propinquity in friendship formation. Journal of Personality and Social Psychology, 32(2), 205.

Narayan, D., \& Cassidy, M. F. (2001). A dimensional approach to measuring social capital: development and validation of a social capital inventory. Current sociology, 49(2), 59102.

Nie, N. H. (2001). Sociability, interpersonal relations, and the internet reconciling conflicting findings. American behavioral scientist, 45(3), 420-435.

O'Reilly, T. (2005, September 30). What is Web 2.0? Design patterns and business models for the next generation of software. Retrieved from http://oreilly.com/web2/archive/what-isweb-20.html.

Padgett, D. \& Allen, D. (1997). Communicating experiences: A narrative approach to creating service brand image. Journal of Advertising, 26(4), 49-62.

Parasuraman, A. (2000). Technology readiness index (TRI): A multiple-item scale to measure readiness to embrace new technologies. Journal of Service Research, 2(4), 307-320.

Park, C. W., Jaworski, B. J., \& MacInnis, D. J. (1986). Strategic brand concept-image management. Journal of Marketing, 50(4), 135-145.

Pelling, M. \& High, C. (2005). Understanding adaptation: What can social capital offer assessments of adaptive capacity? Global Environmental Change, 15(4), 308-319.

Perse, E. M., \& Rubin, R. B. (1989). Attribution in social and parasocial relationships. Communication Research, 16(1), 59-77.

Pezalla, A. E., Pettigrew, J., \& Miller-Day, M. (2012). Researching the researcher-as-instrument: An exercise in interviewer self-reflexivity. Qualitative Research, 12(2), 165-185.

Pine, B. J., \& Gilmore, J. H. (1999). The experience economy: work is theatre \& every business a stage. Harvard Business Press.

Portes, A. (1998). Social capital: Its origins and applications in modern sociology. Annual review of sociology, 24(1), 1-24.

Portes, A. (2000). Social capital: Its origins and applications in modern sociology. LESSER, Eric L. Knowledge and Social Capital. Boston: Butterworth-Heinemann, 43-67.

Pronschinske, M., Groza, M. D., \& Walker, M. (2012). Attracting Facebook 'fans': the importance of authenticity and engagement as a social networking strategy for professional sport teams. Sport marketing quarterly, 21(4), 221. 
Protalinski, E. (2016, April 27). Facebook passes 1.65 billion monthly active users, 54\% access the service only on mobile. Retrieved from http://venturebeat.com/2016/04/27/facebookpasses-1-65-billion-monthly-active-users-54-access-the-service-only-on-mobile/

Putnam, R. D. (1995). Bowling alone: America's declining social capital. Journal of democracy, $6(1), 65-78$.

Putnam, R. D. (2000). Bowling alone: America's declining social capital. In Culture and politics (pp. 223-234). Palgrave Macmillan US.

Putnam, R. D., Leonardi, R., \& Nanetti, R. Y. (1994). Making democracy work: Civic traditions in modern Italy. Princeton university press.

Rashid, S. \& Ghose, K. (2015). Organizational culture and the creation of brand identity: Retail food branding in new markets. Marketing Intelligence and Planning, 33(1), 2-19.

Reisch, M. S. (2016, May 9). Making cosmetic claims that stick. Retrieved from http://cen.acs.org/articles/94/i19/Making-cosmetic-claims-stick.html

Richtman, M. (2015, Dec. 16). The 7 highest paid beauty bloggers of 2015 will make you green with envy - VIDEOS. Retrieved from https://www.bustle.com/articles/129919-the-7highest-paid-beauty-bloggers-of-2015-will-make-you-green-with-envy

Ritchie, J. \& Spencer, L. (1994). Qualitative data analysis for applied policy research. In A. Bryman and R. G. Burgess (Eds.), Analyzing qualitative data (p.173-194). New York: Routledge.

Robbins, I. P. (2013). What Is the Meaning of 'Like'?: The First Amendment Implications of Social-Media Expression.

Roberts, G., \& Sherratt, T. N. (1998). Development of cooperative relationships through increasing investment. Nature, 394(6689), 175-179.

Rocamora, A. (2012). Hypertextuality and remediation in the fashion media: The case of fashion blogs. Journalism Practice, 6(1), 92-106.

Rogers, R. A. (2006). From cultural exchange to transculturation: A review and reconceptualization of cultural appropriation. Communication Theory, 16(4), 474-503.

Rogers, R. (2013). Digital methods. Cambridge: The MIT Press.

Rohm, A., Kaltcheva, V. D., \& Milne, G. R. (2013). A mixed-method approach to examining brand-consumer interactions driven by social media. Journal of Research in Interactive Marketing, 7(4), 296-311.

Rosenberg, M. (1979). Conceiving the self. New York: Basic Books, Inc. 
Rubin, A. M. (2002). The uses-and-gratifications perspectives of media effects. In J. Bryant \& D. Zillman (Eds.), Media effects: Advances in theory and research (2 ${ }^{\text {nd }}$ Ed.) (p. 525-548). Mahwah, NJ: Lawrence Erlbaum Associates Publishers

Rubin, A. M., Perse, E. M., \& Powell, R. A. (1985). Loneliness, parasocial interaction, and local television news viewing. Human Communication Research, 12(2), 155-180.

Sale, J. E., Lohfeld, L. H., \& Brazil, K. (2002). Revising the quantitative-qualitative debate: Implications for mixed-methods research. In V. L. Plano Clark \& J. W. Creswell (Eds.), The mixed methods reader (p. 363-374). Thousand Oaks, California: Sage.

Sander, T., \& Lee, T. P. (2014a). A concept to measure social capital in social network sites. International Journal of Future Computer and Communication, 3(2), 105.

Sander, T., \& Teh, P. L. (2014b, September). Determining the indicators of social capital theory to social network sites. In User Science and Engineering (i-USEr), 2014 3rd International Conference on (pp. 264-268). IEEE.

Schaefer, K. (2016, October 21). Kylie Jenner built a business empire out of lip kits and fan worship. Retrieved from http://www.vanityfair.com/style/2016/10/kylie-jenner-lip-kitsseed-beauty-colourpop

Sedeke, K., \& Arora, P. (2013). Top ranking fashion blogs and their role in the current fashion industry. First Monday, 18(8).

Selfhout, M., Denissen, J., Branje, S., \& Meeus, W. (2009). In the eye of the beholder: perceived, actual, and peer-rated similarity in personality, communication, and friendship intensity during the acquaintanceship process. Journal of personality and social psychology, 96(6), 1152.

Senft, T. M. (2008). Camgirls: Celebrity and community in the age of social networks. New York: Peter Lang Publishing, Inc.

Siegrist, M., Cvetkovich, G., \& Roth, C. (2000). Salient value similarity, social trust, and risk/benefit perception. Risk analysis, 20(3), 353-362.

Silverstein, M., Conroy, S. J., Wang, H., Giarrusso, R., \& Bengtson, V. L. (2002). Reciprocity in parent-child relations over the adult life course. The Journals of Gerontology Series B: Psychological Sciences and Social Sciences, 57(1), S3-S13.

Sirgy, M. J. (1982). Self-concept in consumer behavior: A critical review. Journal of Consumer Research, 9(3), 287-300. 
Sloan, M. M. (2007). The "real self" and inauthenticity: The importance of self-concept anchorage for emotional experiences in the workplace. Social Psychology Quarterly, 70(3), 305-318.

Spencer, S., Harding, J. \& Sheahan, J. (2014). Social eCommerce: Increasing sales and extending brand reach. Sebastopol, CA: O’Reilly Media, Inc.

Srivastava, A. \& Thomson, S. B. (2009).Framework analysis: A qualitative methodology for applied policy research. Journal of Administration \& Governance, 4(2), 72-79.

Steinfield, C., Ellison, N.B., \& Lampe, C. (2008). Social capital, self-esteem, and use of online social network sites: A longitudinal analysis. Journal of Applied Developmental Psychology, 29 (6), 434-445.

Stroebe, W., Insko, C. A., Thompson, V. D., \& Layton, B. D. (1971). Effects of physical attractiveness, attitude similarity, and sex on various aspects of interpersonal attraction. Journal of personality and social psychology, 18(1), 79.

Strugatz, R. (2016, August 10). Bloggers and digital influencers are reshaping the fashion and beauty landscape. Retrieved from http://www.latimes.com/fashion/la-ig-bloggers20160809-snap-story.html

Sweeney, J. C., Soutar, G. N., \& Mazzarol, T. (2008). Factors influencing word of mouth effectiveness: receiver perspectives. European Journal of Marketing, 42(3/4), 344-364.

Swol, L. M., \& Sniezek, J. A. (2005). Factors affecting the acceptance of expert advice. British Journal of Social Psychology, 44(3), 443-461.

Szabo, G., \& Huberman, B. A. (2010). Predicting the popularity of online content. Communications of the ACM, 53(8), 80-88.

Tashakkori, A., \& Teddlie, C. (1998). The evolution of mixed methods research. In V. L. Plano Clark \& J. W. Creswell (Eds.), The mixed methods reader (p. 7-26). Thousand Oaks, California: Sage.

Terpening, E., Li, C. \& Akhtar, O. (2015, July 28). The 2015 state of social businesses:

Priorities shift from scaling to integrating. Retrieved from http://www.altimetergroup.com/pdf/reports/2015-State-Of-Social-Business-AltimeterGroup.pdf

Tripathi, R. C., Caplan, R. D., \& Naidu, R. K. (1986). Accepting advice: A modifier of social support's effect on well-being. Journal of Social and Personal Relationships, 3(2), 213228.

Turato, E. R. (2005). Qualitative and quantitative methods in health: definitions, differences and research subjects. Revista de Saúde Pública, 39(3), 507-514. 
Turkle, S. (2012). Alone together: Why we expect more from technology and less from each other. New York: Basic Books.

Twitter. (2016). Twitter usage/company facts. Retrieved from https://about.twitter.com/company

Valenzuela, S., Park, N., \& Kee, K. F. (2009). Is there social capital in a social network site?: Facebook use and college students' life satisfaction, trust, and participation. Journal of Computer-Mediated Communication, 14(4), 875-901.

van Dijck, J. (2009). Users like you? Theorizing agency in user-generated content. Media, Culture \& Society, 31(1), 41-58.

van Dijck, J. (2013). The culture of connectivity: A critical history of social media. New York: Oxford University Press.

Varis, P. (2016). Digital ethnography. In A. Georgakopoulou \& T. Spilioti (Eds.), The Routledge handbook of language and digital communication (p. 55-68). New York: Routledge.

VidStatsX. (2016, December 6). YouTube 100 most subscribed "how to and style" channels list - Top by subscribers. Retrieved from https://vidstatsx.com/youtube-top-100-mostsubscribed-how-to-style-channels

Wellman, B. (2001). Computer networks as social networks. Science, 293(September), 20312034.

Williams, D. (2006). On and off the 'Net: Scales for social capital in an online era. Journal of Computer-Mediated Communication, 11(2), 593-628.

Wood, L. (2000). Brands and brand equity: Definition and management. Management Decision, 38(9), 662-669.

Wu, K. (2016). YouTube marketing: Legality of sponsorship and endorsement in advertising. Journal of Law, Business, and Ethics, 22(2016), 59-91.

YouTube. (2016a). All categories - Results for “beauty”. Retrieved from https://www.youtube.com/channels?q=beauty

YouTube. (2016b). Statistics. Retrieved from https://www.youtube.com/yt/press/statistics.html

YouTube. (2016c). YouTube partner program overview. Retrieved from https://support.google.com/youtube/answer/72851?hl=en

Zimmer, M. (2010). "But the data is already public": on the ethics of research in Facebook. Ethics and information technology, 12(4), 313-325. 
